Supporting information

\title{
Catalytic Vicinal Dichlorination of Unactivated Alkenes
}

Jérôme C. Sarie, Jessica Neufeld, Constantin G. Daniliuc and Ryan Gilmour

Organisch-Chemisches Institut, WWU Münster, Corrensstr. 40, 48149 Münster, Germany.

E-mail: ryan.gilmour@uni-muenster.de 


\section{Contents}

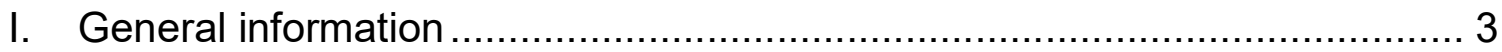

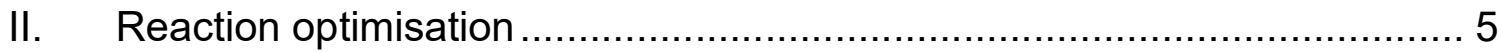

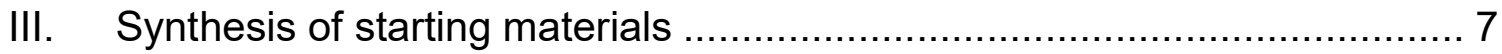

IV. General procedure B for the catalytic dichlorination of terminal alkenes .. 22

V. General procedure C for the catalytic dichlorination of internal alkenes... 34

VI. Mechanistic studies on the dichlorination of terminal alkenes ................ 39

VII. Preliminary validation of an enantioselective variant ........................... 48

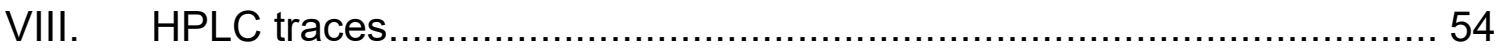

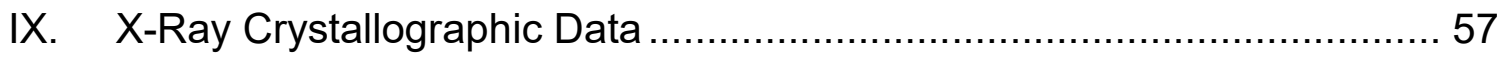

X. NMR Spectra of unreported starting materials ................................. 63

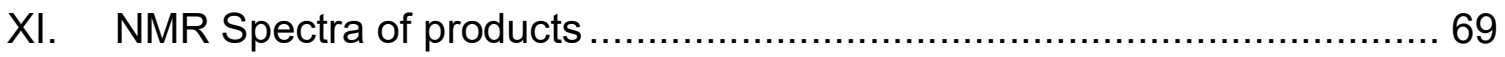

XII. NMR Spectra: preliminary validation of an enantioselective variant .........89 89

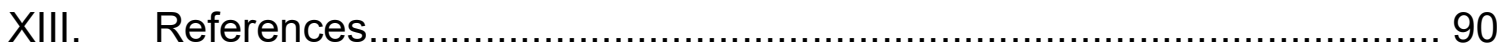




\section{General information}

All chemicals were purchased as reagent grade and used as received except for 4chlorostyrene and 11-bromo-1-undecene which were filtered through silica using 100\% $n$-pentane prior to use. Dry solvents were dried by a Grubbs purification system including columns packed with molecular sieves and aluminium oxide. Solvents for extraction or purification were purchased as technical grade and distilled on the rotary evaporator prior to use (exception: diethyl ether was used as purchased). Column chromatography was performed using silica gel (40-63 $\mu \mathrm{m}$; VWR Chemicals) as stationary phase. Reaction monitoring was achieved by analytical thin layer chromatography (TLC) on aluminum foil pre-coated with silica gel 60 F254 (Merck). Compounds were visualised with UV light $(254 \mathrm{~nm})$ or by chemical staining using solution of $\mathrm{KMnO}_{4}\left(\mathrm{KMnO}_{4}(10 \mathrm{~g}), \mathrm{K}_{2} \mathrm{CO}_{3}(65 \mathrm{~g}), \mathrm{NaOH}(1 \mathrm{~N}, 15 \mathrm{~mL})\right.$ in water $\left.(1 \mathrm{~L})\right)$ or of CAM $\left(\left(\mathrm{NH}_{4}\right)_{6} \mathrm{Mo}_{7} \mathrm{O}_{24} \cdot 4 \mathrm{H}_{2} \mathrm{O}(50 \mathrm{~g}), \mathrm{Ce}\left(\mathrm{SO}_{4}\right)_{2}(10 \mathrm{~g})\right.$, conc. $\mathrm{H}_{2} \mathrm{SO}_{4}(100 \mathrm{~mL})$ in water $(900 \mathrm{~mL}))$ followed by heating. Concentration in vacuo was performed at $\sim 10$ mbar at $40{ }^{\circ} \mathrm{C}$ unless otherwise stated. NMR spectra were measured by the NMR service in the Organisch-Chemisches Institut, Westfälische Wilhelms-Universität Münster on a Bruker Avance I/ 300, a Bruker Avance II 400, an Agilent DD2 500 or an Agilent DD2 600 spectrometer at $298 \mathrm{~K} .{ }^{1} \mathrm{H}$ NMR chemical shifts are given relative to TMS and are referenced to the residual solvent peak as internal standard. Spectra of other nuclides as ${ }^{13} \mathrm{C}$ and ${ }^{19} \mathrm{~F}$ are referenced according to the proton resonance of TMS as the primary reference for the unified chemical shift scale. ${ }^{1} \mathrm{H}$ NMR spectra are reported as follows: chemical shift $\delta$ in ppm (multiplicity, coupling constant $J_{\mathrm{PH}}, J_{\mathrm{FH}}$ and $\mathrm{JHH}_{\mathrm{H}}$ in $\mathrm{Hz}$, number of protons, assignment of proton). ${ }^{13} \mathrm{C}$ NMR spectra are reported as follows: chemical shift $\delta$ in ppm (multiplicity, coupling constant $J_{\mathrm{PC}}, J_{\mathrm{FC}}$ in $\mathrm{Hz}$, number of carbons, assignment of carbon). ${ }^{19} \mathrm{~F}$ NMR spectra are reported as follows: chemical shift $\delta$ in ppm (multiplicity, coupling constant $J_{\mathrm{FH}}$ in $\mathrm{Hz}$, number of fluorines, assignment 
of fluorine). The resonance multiplicity is abbreviated as $s$ (singlet), d (doublet), $t$ (triplet), $q$ (quartet), $p$ (pentet) or $m$ (multiplet). Assignments of unknown compounds are based on COSY, HMBC and HSQC spectra. Melting points were measured on a Büchi B-545 melting point apparatus in open capillaries and are uncorrected. IR spectra were recorded on a Perkin-Elmer 100 FT-IR spectrometer. Absorption bands are reported in wave numbers $\left(\mathrm{cm}^{-1}\right)$ and the intensities are reported as $\mathrm{w}$ (weak), $\mathrm{m}$ (medium), $\mathrm{s}$ (strong). Mass spectra were measured by the MS service of the Organisch-Chemisches Institut, Westfälische Wilhelms-Universität on a Bruker Daltonics MicroTof (HRMS-ESI), a Triplequad TSQ 7000 (MS-EI), Triplequad Quattro Micro GC (GC-EI-MS), a Qp5050 Single Quad (GC-EI-MS) or a LTQ Orbitap LTQ XL (HRMS-APCI). Enantiomeric ratios were determined on an Agilent Infinity 1260 HPLC system using a diode array detector (DAD). The chiral stationary phases were OJ, OM and AM. The eluent of $n$-hexane and $i$-propanol is specified for each compound. The column temperature measured 25 to $35^{\circ} \mathrm{C}$. 


\section{Reaction optimisation}

a. Table S1: Exploring alternative oxidants.

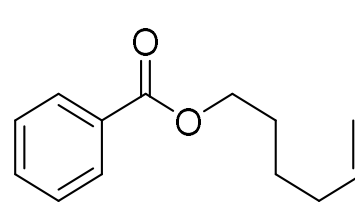

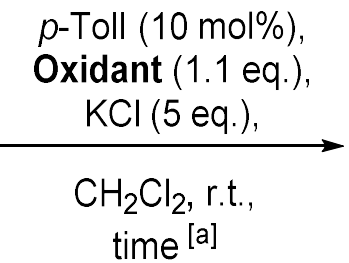<smiles>O=C(OCCCC(Cl)CCl)c1ccccc1</smiles>

\begin{tabular}{|c|c|c|c|c|}
\hline Entry & Oxidant & $\mathrm{MCl}$ & time (h) & Yield $^{[a]}$ \\
\hline 1 & Ground oxone ${ }^{\circledR}$ & $\mathrm{KCl}$ & 15 & $67 \%$ \\
\hline $2^{[b]}$ & Ground oxone ${ }^{\circledR}$ & $\mathrm{KCl}$ & 15 & $70 \%$ \\
\hline 3 & $m \mathrm{CPBA}$ & $\mathrm{KCl}$ & 15 & $5 \%[c]$ \\
\hline 4 & aq. $\mathrm{NaOCl} 10 \%$ & $\mathrm{KCl}$ & 15 & $<5 \%$ \\
\hline 5 & $\mathrm{NaIO}_{4}$ & $\mathrm{KCl}$ & 15 & $<5 \%$ \\
\hline 6 & CAN & $\mathrm{KCl}$ & 15 & $<5 \%$ \\
\hline 7 & $t-\mathrm{BuOOH}$ & $\mathrm{KCl}$ & 15 & $<5 \%$ \\
\hline 8 & aq. $\mathrm{H}_{2} \mathrm{O}_{2} 30 \%$ & $\mathrm{KCl}$ & 15 & $<5 \%$ \\
\hline 9 & $\mathrm{~K}_{2} \mathrm{~S}_{2} \mathrm{O}_{8}$ & $\mathrm{KCl}$ & 15 & $<5 \%$ \\
\hline 10 & $\mathrm{CH}_{3} \mathrm{CO}_{3} \mathrm{H}$ & $\mathrm{KCl}$ & 15 & $<5 \%$ \\
\hline 11 & $\mathrm{CH}_{3} \mathrm{CO}_{3} \mathrm{H}$ & TMSCI & 15 & $40 \%$ \\
\hline $12^{[b]}$ & $\mathrm{CH}_{3} \mathrm{CO}_{3} \mathrm{H}$ & TMSCI & 15 & $46 \%$ \\
\hline 13 & Selectfluor ${ }^{\circledR}$ & $\mathrm{KCl}$ & 15 & $<5 \%$ \\
\hline $14^{[\mathrm{d}]}$ & Selectfluor $^{\circledR}$ & $\mathrm{KCl}$ & 3 & $59 \%$ \\
\hline $15^{[b][d]}$ & Selectfluor ${ }^{\circledR}$ & $\mathrm{KCl}$ & 3 & $<5 \%$ \\
\hline
\end{tabular}

[a] ${ }^{1} \mathrm{H}$ NMR yield using DMF as internal standard. [b] Control experiment without catalyst. [c] Epoxidation of the alkene was observed. The analytic data of the isolated product are in agreement with the literature. ${ }^{1}$ [d] HFIP instead of $\mathrm{CH}_{2} \mathrm{Cl}_{2}$. 
b. Table S2: Interrogating the role of HFIP as an additive.

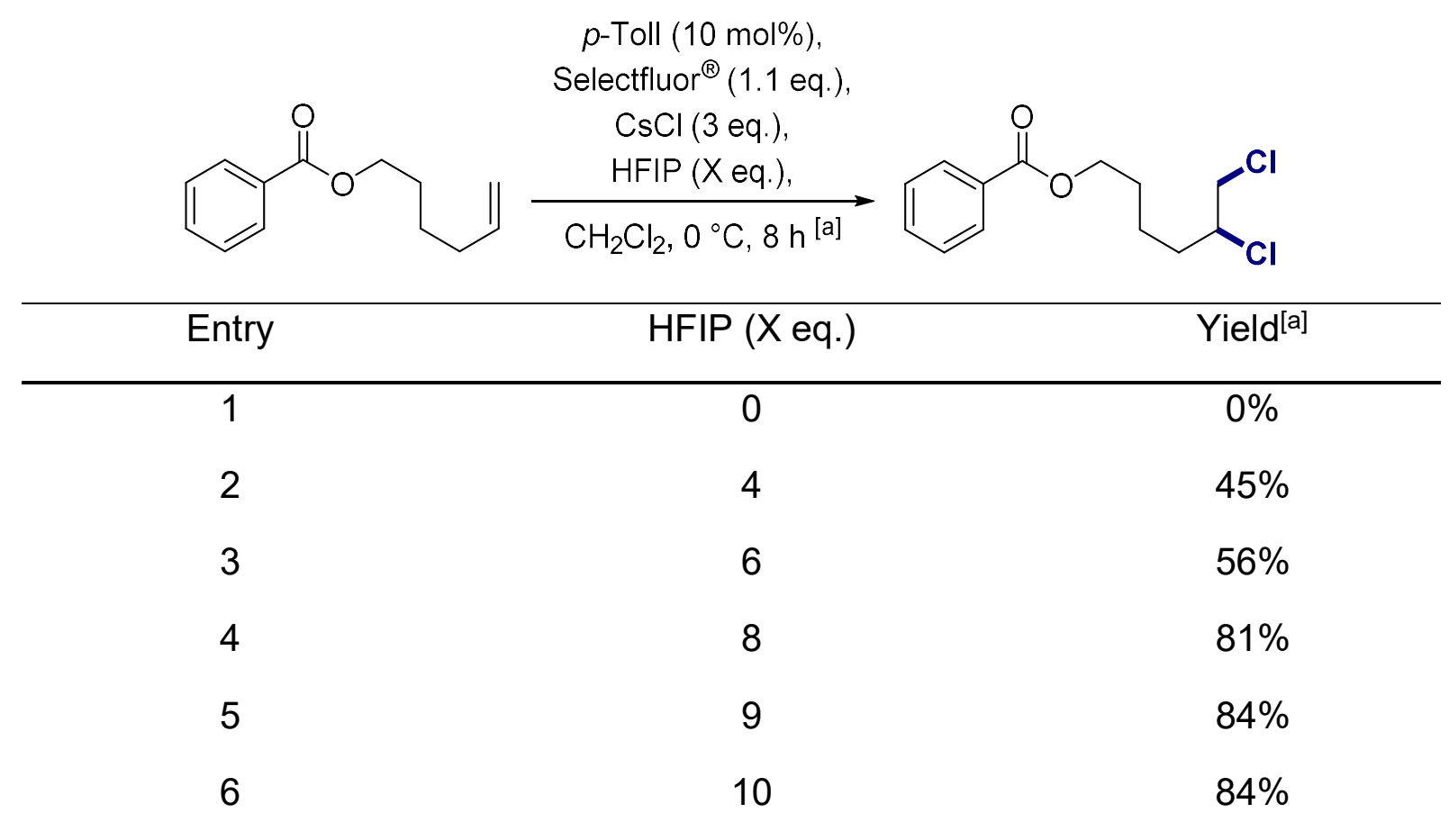

[a] ${ }^{1} \mathrm{H}$ NMR yield using DMF as internal standard.

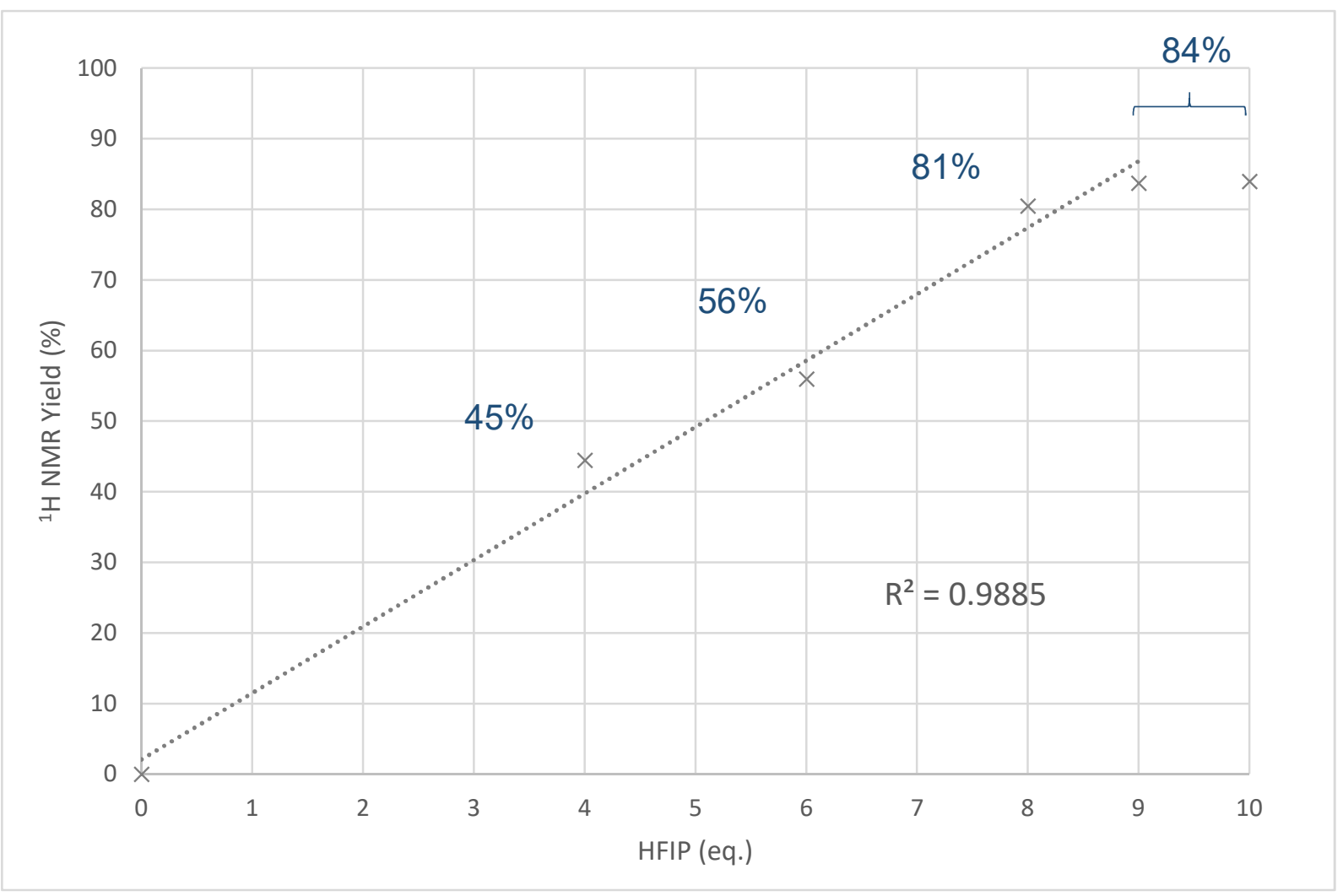

Figure S1: Dependency of the ${ }^{1} \mathrm{H}$ NMR yield in function of HFIP. 


\section{Synthesis of starting materials}

\section{General procedure A for the esterification of acyl chlorides with alkenols}

The corresponding alkenol (1.0 eq.) and acyl chloride (1.1-2.0 eq.) were dissolved in diethyl ether. Then NEt 3 (1.1-2.0 eq.) was added slowly and the reaction mixture was stirred for the indicated time (monitored by TLC) at ambient temperature. The reaction mixture was diluted with ethyl acetate and water, and the aqueous layer was extracted with ethyl acetate $(3 \mathrm{x})$. The combined organic layers were washed with water and brine, dried over $\mathrm{MgSO}_{4}$ and filtered. The solvent was removed in vacuo and the crude mixture was purified by column chromatography.

\section{Hex-5-en-1-yl benzoate (1)}

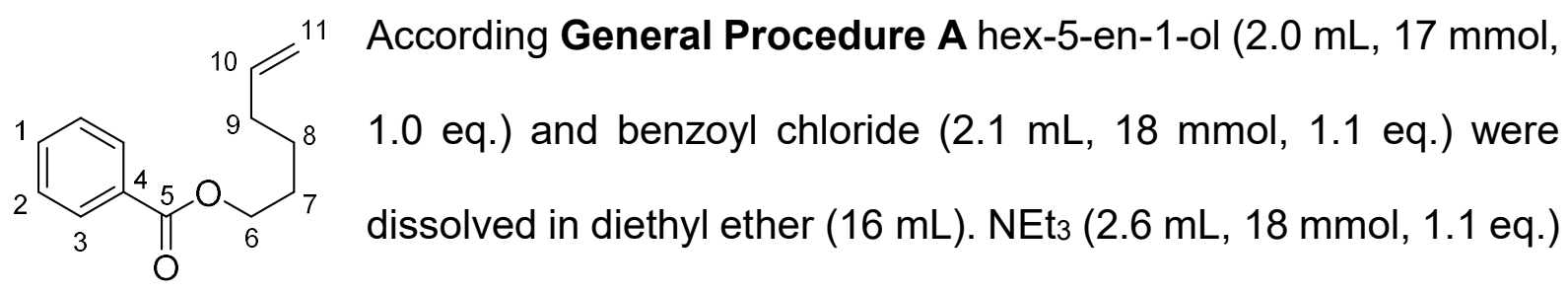
was added slowly and the reaction mixture was stirred for $19 \mathrm{~h}$. The crude mixture was purified by column chromatography using cyclohexane:ethyl acetate $98: 2$ to afford hex5-en-1-yl benzoate (1) as a colourless oil (3.1 g, $15 \mathrm{mmol}, 92 \%)$.

Rf: (cyclohexane:ethyl acetate 95:5) 0.32;

${ }^{1} \mathrm{H}$ NMR $\left(400 \mathrm{MHz}, \mathrm{CDCl}_{3}\right) \delta=8.05\left(\mathrm{~m}, 2 \mathrm{H}, \mathrm{H}^{3}\right), 7.55\left(\mathrm{~m}, 1 \mathrm{H}, \mathrm{H}^{1}\right), 7.44\left(\mathrm{~m}, 2 \mathrm{H}, \mathrm{H}^{2}\right)$, $5.82\left(\mathrm{ddt},{ }^{3} \mathrm{JHH}_{\mathrm{HH}}=16.9,10.3,6.7 \mathrm{~Hz}, 1 \mathrm{H}, \mathrm{H}^{10}\right), 5.04\left(\mathrm{td},{ }^{3} \mathrm{JHH}_{\mathrm{HH}}=16.9 \mathrm{~Hz},{ }^{2} \mathrm{~J}_{\mathrm{HH}}=1.8 \mathrm{~Hz}\right.$, $\left.1 \mathrm{H}, \mathrm{H}^{11 E}\right), 4.98\left(\mathrm{td},{ }^{3} \mathrm{~J}_{\mathrm{HH}}=10.3 \mathrm{~Hz},{ }^{2} \mathrm{~J}_{\mathrm{HH}}=1.8 \mathrm{~Hz}, 1 \mathrm{H}, \mathrm{H}^{11 \mathrm{z}}\right), 4.33\left(\mathrm{t},{ }^{3} \mathrm{JHH}=6.6 \mathrm{~Hz}, 2 \mathrm{H}\right.$, $\left.\mathrm{H}^{6}\right), 2.14\left(\mathrm{td},{ }^{3} \mathrm{JHH}_{\mathrm{HH}}=7.6,6.7 \mathrm{~Hz}, 2 \mathrm{H}, \mathrm{H}^{9}\right), 1.82\left(\mathrm{tt},{ }^{3} \mathrm{~J}_{\mathrm{HH}}=7.4,6.6 \mathrm{~Hz}, 2 \mathrm{H}, \mathrm{H}^{7}\right), 1.58(\mathrm{tt}$, $\left.{ }^{3} \mathrm{JHH}_{\mathrm{HH}}=7.6,7.4 \mathrm{~Hz}, 2 \mathrm{H}, \mathrm{H}^{8}\right) \mathrm{ppm}$; 
${ }^{13} \mathrm{C}\left\{{ }^{1} \mathrm{H}\right\}$ NMR $\left(101 \mathrm{MHz}, \mathrm{CDCl}_{3}\right) \delta=166.8\left(1 \mathrm{C}, \mathrm{C}^{5}\right), 138.5\left(1 \mathrm{C}, \mathrm{C}^{10}\right), 132.9\left(1 \mathrm{C}, \mathrm{C}^{1}\right)$, $130.6\left(1 \mathrm{C}, \mathrm{C}^{4}\right), 129.7\left(2 \mathrm{C}, \mathrm{C}^{3}\right), 128.4\left(2 \mathrm{C}, \mathrm{C}^{2}\right), 115.0$ (1C, C $\left.\mathrm{C}^{11}\right), 65.0\left(1 \mathrm{C}, \mathrm{C}^{6}\right), 33.4$ $\left(1 \mathrm{C}, \mathrm{C}^{9}\right), 28.3\left(1 \mathrm{C}, \mathrm{C}^{7}\right), 25.4\left(1 \mathrm{C}, \mathrm{C}^{8}\right) \mathrm{ppm}$;

IR (ATR) $\tilde{v}=3075(w), 2936$ (w), $2860(w), 1716(s), 1640(w), 1602(w), 1585(w)$, $1451(\mathrm{~m}), 1385(\mathrm{w}), 1314(\mathrm{~m}), 1268(\mathrm{~s}), 1175(\mathrm{~m}), 1109(\mathrm{~s}), 1069(\mathrm{~m}), 1026(\mathrm{~m}), 993$ (m), $910(\mathrm{~m}), 805(\mathrm{w}), 707(\mathrm{~s}), 686(\mathrm{~m}) \mathrm{cm}^{-1}$;

HRMS (ESI) $m / z$ [M+Na] calcd. for $\mathrm{C}_{13} \mathrm{H}_{16} \mathrm{O}_{2} \mathrm{Na}^{+}: 227.1043$, found: 227.1040 .

The analytic data are in agreement with the literature. ${ }^{2}$

(E)-Hex-4-en-1-yl benzoate (S1)

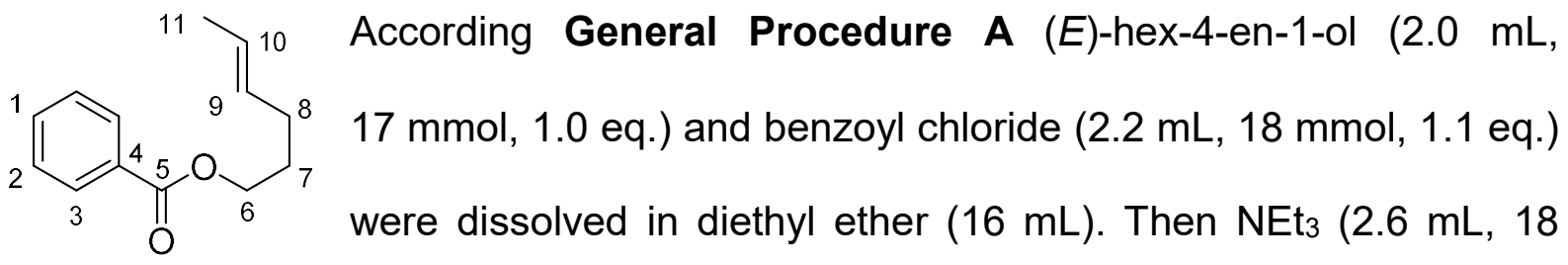
mmol, 1.1 eq.) was added slowly and the reaction mixture was stirred for $19 \mathrm{~h}$. The crude mixture was purified by column chromatography using cyclohexane:ethyl acetate $98: 2$ to afford (E)-hex-4-en-1-yl benzoate (S1) as a colourless oil $(3.2 \mathrm{~g}, 16$ mmol, 94\%).

Rf: (n-pentane:diethyl ether 10:1) 0.69 ;

${ }^{1} \mathrm{H}$ NMR $\left(599 \mathrm{MHz}, \mathrm{CDCl}_{3}\right) \delta=8.05\left(\mathrm{~m}, 2 \mathrm{H}, \mathrm{H}^{3}\right), 7.55\left(\mathrm{~m}, 1 \mathrm{H}, \mathrm{H}^{1}\right), 7.44\left(\mathrm{~m}, 2 \mathrm{H}, \mathrm{H}^{2}\right)$, $5.53-5.39\left(\mathrm{~m}, 2 \mathrm{H}, \mathrm{H}^{9}, \mathrm{H}^{10}\right), 4.32\left(\mathrm{t},{ }^{3} \mathrm{~J}_{\mathrm{HH}}=6.7 \mathrm{~Hz}, 2 \mathrm{H}, \mathrm{H}^{6}\right), 2.15\left(\mathrm{tdd},{ }^{3} \mathrm{~J}_{\mathrm{HH}}=7.7\right.$, $\left.6.1 \mathrm{~Hz},{ }^{4} \mathrm{JHH}_{\mathrm{HH}}=1.5 \mathrm{~Hz}, 2 \mathrm{H}, \mathrm{H}^{8}\right), 1.83\left(\mathrm{tt},{ }^{3} \mathrm{~J}_{\mathrm{HH}}=7.7,6.7 \mathrm{~Hz}, 2 \mathrm{H}, \mathrm{H}^{7}\right), 1.67-1.64(\mathrm{~m}, 3 \mathrm{H}$, $\left.\mathrm{H}^{11}\right) \mathrm{ppm}$; 
${ }^{13} \mathrm{C}\left\{{ }^{1} \mathrm{H}\right\}$ NMR $\left(151 \mathrm{MHz}, \mathrm{CDCl}_{3}\right) \delta=166.8\left(1 \mathrm{C}, \mathrm{C}^{5}\right), 132.9\left(1 \mathrm{C}, \mathrm{C}^{1}\right), 130.6\left(1 \mathrm{C}, \mathrm{C}^{4}\right)$, $130.1\left(1 \mathrm{C}, \mathrm{C}^{9}\right), 129.7\left(2 \mathrm{C}, \mathrm{C}^{3}\right), 128.4\left(2 \mathrm{C}, \mathrm{C}^{2}\right), 126.0$ (1C, $\left.\mathrm{C}^{10}\right), 64.6\left(1 \mathrm{C}, \mathrm{C}^{6}\right), 29.1$ (1C, $\left.\mathrm{C}^{8}\right), 28.7\left(1 \mathrm{C}, \mathrm{C}^{7}\right), 18.0\left(1 \mathrm{C}, \mathrm{C}^{11}\right) \mathrm{ppm}$;

IR (ATR) $\tilde{v}=2937(w), 1716(s), 1602(w), 1585(w), 1450(m), 1385(w), 1314(m)$, $1268(\mathrm{~s}), 1175(\mathrm{~m}), 1109(\mathrm{~s}), 1069(\mathrm{~m}), 1026(\mathrm{~m}), 965(\mathrm{~m}), 927(\mathrm{w}), 806(\mathrm{w}), 707(\mathrm{~s})$, $686(\mathrm{~m}) \mathrm{cm}^{-1}$;

HRMS (ESI) $m / z$ [M+Na] calcd. for $\mathrm{C}_{13} \mathrm{H}_{16} \mathrm{O}_{2} \mathrm{Na}^{+}: 227.1043$, found: 227.1049 .

The analytic data are in agreement with the literature. ${ }^{3}$

\section{(Z)-Hex-4-en-1-yl benzoate (S2)}

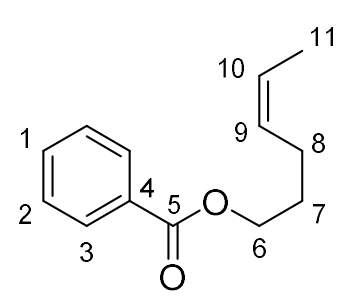

According General Procedure A (Z)-hex-4-en-1-ol (1.0 mL, $8.3 \mathrm{mmol}, 1.0$ eq.) and benzoyl chloride $(1.1 \mathrm{~mL}, 9.2 \mathrm{mmol}$, 1.1 eq.) were dissolved in diethyl ether $(8 \mathrm{~mL})$. Then $\mathrm{NEt}_{3}(1.3 \mathrm{~mL}$, $9.2 \mathrm{mmol}, 1.1 \mathrm{eq}$.$) was added slowly and the reaction mixture was$ stirred for $19 \mathrm{~h}$. The crude mixture was purified by column chromatography using cyclohexane:ethyl acetate 98:2 to afford (Z)-hex-4-en-1-yl benzoate (S2) as a colourless oil $(1.0 \mathrm{~g}, 5.1 \mathrm{mmol}, 61 \%)$.

Rf: ( $n$-pentane:diethyl ether 10:1) 0.69;

${ }^{1} \mathrm{H}$ NMR $\left(599 \mathrm{MHz}, \mathrm{CDCl}_{3}\right) \delta=8.06\left(\mathrm{~m}, 2 \mathrm{H}, \mathrm{H}^{3}\right), 7.56\left(\mathrm{~m}, 1 \mathrm{H}, \mathrm{H}^{1}\right), 7.44\left(\mathrm{~m}, 2 \mathrm{H}, \mathrm{H}^{2}\right)$, $5.52\left(\mathrm{dqt},{ }^{3} \mathrm{JHH}_{\mathrm{HH}}=10.8,6.6 \mathrm{~Hz},{ }^{4} \mathrm{JHH}_{\mathrm{HH}}=1.5 \mathrm{~Hz}, 1 \mathrm{H}, \mathrm{H}^{10}\right), 5.42\left(\mathrm{dtq},{ }^{3} \mathrm{~J}_{\mathrm{HH}}=10.8,7.2 \mathrm{~Hz}\right.$, $\left.{ }^{4} J_{\mathrm{HH}}=1.7 \mathrm{~Hz}, 1 \mathrm{H}, \mathrm{H}^{9}\right), 4.33\left(\mathrm{t},{ }^{3}{ }_{\mathrm{HH}}=6.6 \mathrm{~Hz}, 2 \mathrm{H}, \mathrm{H}^{6}\right), 2.22$ (tddq, ${ }^{3} J_{\mathrm{HH}}=7.4,7.2 \mathrm{~Hz}$, $\left.{ }^{4} \mathrm{~J}_{\mathrm{HH}}=1.6 \mathrm{~Hz},{ }^{5} \mathrm{JHH}_{\mathrm{HH}}=0.9 \mathrm{~Hz}, 2 \mathrm{H}, \mathrm{H}^{8}\right), 1.85\left(\mathrm{tt},{ }^{3} \mathrm{~J}_{\mathrm{HH}}=7.4,6.6 \mathrm{~Hz}, 2 \mathrm{H}, \mathrm{H}^{7}\right), 1.62(\mathrm{ddt}$, $\left.{ }^{3} \mathrm{JHH}_{\mathrm{HH}}=6.8 \mathrm{~Hz},{ }^{4} \mathrm{~J}_{\mathrm{HH}}=1.7 \mathrm{~Hz},{ }^{5} \mathrm{JHH}=0.9 \mathrm{~Hz}, 3 \mathrm{H}, \mathrm{H}^{11}\right) \mathrm{ppm}$; 
${ }^{13} \mathrm{C}\left\{{ }^{1} \mathrm{H}\right\} \operatorname{NMR}\left(151 \mathrm{MHz}, \mathrm{CDCl}_{3}\right) \delta=166.8\left(1 \mathrm{C}, \mathrm{C}^{5}\right), 132.9\left(1 \mathrm{C}, \mathrm{C}^{1}\right), 130.6\left(1 \mathrm{C}, \mathrm{C}^{4}\right)$, $129.7\left(2 \mathrm{C}, \mathrm{C}^{3}\right), 129.2\left(1 \mathrm{C}, \mathrm{C}^{9}\right), 128.5\left(2 \mathrm{C}, \mathrm{C}^{2}\right), 125.2\left(1 \mathrm{C}, \mathrm{C}^{10}\right), 64.6\left(1 \mathrm{C}, \mathrm{C}^{6}\right)$, $28.7\left(1 \mathrm{C}, \mathrm{C}^{7}\right), 23.4\left(1 \mathrm{C}, \mathrm{C}^{8}\right), 12.9\left(1 \mathrm{C}, \mathrm{C}^{11}\right) \mathrm{ppm}$;

IR (ATR) $\tilde{v}=3014(w), 2953(w), 1716(s), 1602(w), 1585(w), 1451(m), 1386(w)$, $1314(m), 1268(s), 1175(m), 1109(s), 1069(m), 1026(m), 997(w), 966(w), 806(w)$, $707(\mathrm{~s}), 686(\mathrm{~m}) \mathrm{cm}^{-1}$;

HRMS (ESI) $m / z$ [M+Na] $]^{+}$calcd. for $\mathrm{C}_{13} \mathrm{H}_{16} \mathrm{O}_{2} \mathrm{Na}^{+}: 227.1043$, found: 227.1039 .

The analytic data are in agreement with the literature. ${ }^{4}$

\section{(E)-Hex-4-en-1-yl 4-methoxybenzoate (S3)}

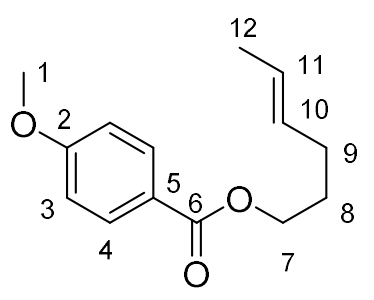

According General Procedure A (E)-hex-4-en-1-ol $(0.59 \mathrm{~mL}$, $5.0 \mathrm{mmol}, 1.0$ eq.) and 4-methoxybenzoyl chloride $(0.78 \mathrm{~mL}$, $5.5 \mathrm{mmol}, 1.1$ eq.) were dissolved in diethyl ether $(6 \mathrm{~mL})$. Then $\mathrm{NEt}_{3}(0.77 \mathrm{~mL}, 5.5 \mathrm{mmol}, 1.1$ eq.) was added slowly and the reaction mixture was stirred for $19 \mathrm{~h}$. The crude mixture was purified by column chromatography using cyclohexane:ethyl acetate 98:2 to afford (E)-hex-4-en-1-yl 4methoxybenzoate (S3) as a colourless oil (0.82 g, $3.5 \mathrm{mmol}, 63 \%)$.

Rf: (n-pentane:diethyl ether 10:1) 0.41;

${ }^{1} \mathrm{H}$ NMR $\left(599 \mathrm{MHz}, \mathrm{CDCl}_{3}\right) \delta=8.01\left(\mathrm{~m}, 2 \mathrm{H}, \mathrm{H}^{4}\right), 6.91\left(\mathrm{~m}, 2 \mathrm{H}, \mathrm{H}^{3}\right), 5.52-5.41(\mathrm{~m}, 2 \mathrm{H}$, $\left.\mathrm{H}^{10}, \mathrm{H}^{11}\right), 4.28\left(\mathrm{t},{ }^{3} \mathrm{~J}_{\mathrm{HH}}=6.6 \mathrm{~Hz}, 2 \mathrm{H}, \mathrm{H}^{7}\right), 3.85\left(\mathrm{~s}, 3 \mathrm{H}, \mathrm{H}^{1}\right), 2.17-2.07\left(\mathrm{~m}, 2 \mathrm{H}, \mathrm{H}^{9}\right)$, $1.81\left(\mathrm{tt},{ }^{3} \mathrm{~J}_{\mathrm{HH}}=7.9,6.6 \mathrm{~Hz}, 2 \mathrm{H}, \mathrm{H}^{8}\right), 1.68-1.62\left(\mathrm{~m}, 3 \mathrm{H}, \mathrm{H}^{12}\right) \mathrm{ppm}$;

${ }^{13} \mathrm{C}\left\{{ }^{1} \mathrm{H}\right\}$ NMR $\left(151 \mathrm{MHz}, \mathrm{CDCl}_{3}\right) \delta=166.5\left(1 \mathrm{C}, \mathrm{C}^{6}\right), 163.4\left(1 \mathrm{C}, \mathrm{C}^{5}\right), 131.7\left(2 \mathrm{C}, \mathrm{C}^{4}\right)$, $130.2\left(1 \mathrm{C}, \mathrm{C}^{10}\right), 126.0\left(1 \mathrm{C}, \mathrm{C}^{11}\right), 123.1\left(1 \mathrm{C}, \mathrm{C}^{2}\right), 113.7\left(2 \mathrm{C}, \mathrm{C}^{3}\right), 64.3\left(1 \mathrm{C}, \mathrm{C}^{7}\right)$, $55.5\left(1 \mathrm{C}, \mathrm{C}^{1}\right), 29.2\left(1 \mathrm{C}, \mathrm{C}^{9}\right), 28.7\left(1 \mathrm{C}, \mathrm{C}^{8}\right), 18.0\left(1 \mathrm{C}, \mathrm{C}^{12}\right) \mathrm{ppm}$ 
IR (ATR) $\tilde{v}=2937(\mathrm{w}), 2284(\mathrm{w}), 1708(\mathrm{~s}), 1605(\mathrm{~s}), 1510(\mathrm{~m}), 1457(\mathrm{w}), 1385(\mathrm{w})$, $1315(\mathrm{w}), \quad 1271(\mathrm{~s}), \quad 1250(\mathrm{~s}), \quad 1100(\mathrm{~s}), \quad 1028(\mathrm{~s}), \quad 965(\mathrm{~m}), \quad 846(\mathrm{~m}), \quad 768(\mathrm{~s})$, $695(\mathrm{~m}) \mathrm{cm}^{-1}$;

HRMS (ESI) $m / z$ [M+Na] ${ }^{+}$calcd. for $\mathrm{C}_{14} \mathrm{H}_{18} \mathrm{O}_{3} \mathrm{Na}^{+}: 257.1148$, found: 257.1158 .

\section{(E)-Hex-4-en-1-yl 3,5-dinitrobenzoate (S4)}

According General Procedure $\mathbf{A}(E)$-hex-4-en-1-ol $(0.94 \mathrm{~mL}$,
$8.0 \mathrm{mmol}, 1.0$ eq. $)$ and 3,5 -dinitrobenzoyl chloride $(3.6 \mathrm{~g}$, $\mathrm{NEt}_{3}(2.2 \mathrm{~mL}, 16 \mathrm{mmol}, 2.0$ eq.) was added slowly and the reaction mixture was stirred for 5 days. The crude mixture was purified by column chromatography using cyclohexane:ethyl acetate $98: 2$ to afford (E)-hex-4-en-1-yl 3,5-dinitrobenzoate (S4) as a yellow solid (1.7 g, $5.7 \mathrm{mmol}, 71 \%)$.

Rf: (n-pentane:diethyl ether 10:1) 0.34;

M.p. $36-38^{\circ} \mathrm{C}$;

${ }^{1} \mathrm{H}$ NMR $\left(400 \mathrm{MHz}, \mathrm{CDCl}_{3}\right) \delta=9.21\left(\mathrm{~m}, 1 \mathrm{H}, \mathrm{H}^{1}\right), 9.14\left(\mathrm{~m}, 2 \mathrm{H} ; \mathrm{H}^{3}\right), 5.61-5.34(\mathrm{~m}, 2 \mathrm{H}$, $\left.\mathrm{H}^{9}, \mathrm{H}^{10}\right), 4.45\left(\mathrm{t},{ }^{3} \mathrm{JHH}_{\mathrm{HH}}=6.6 \mathrm{~Hz}, 2 \mathrm{H}, \mathrm{H}^{6}\right), 2.16\left(\mathrm{tdd},{ }^{3} \mathrm{JHH}_{\mathrm{HH}}=7.0,6.6, \mathrm{~Hz},{ }^{4} \mathrm{~J}_{\mathrm{HH}}=1.5 \mathrm{~Hz}\right.$, $\left.2 \mathrm{H}, \mathrm{H}^{8}\right), 1.89\left(\mathrm{tt},{ }^{3} \mathrm{~J}_{\mathrm{HH}}=7.0,6.6 \mathrm{~Hz}, 2 \mathrm{H}, \mathrm{H}^{7}\right), 1.65\left(\mathrm{~d},{ }^{3} \mathrm{JHH}_{\mathrm{HH}}=5.5 \mathrm{~Hz}, 3 \mathrm{H}, \mathrm{H}^{11}\right) \mathrm{ppm}$;

${ }^{13} \mathrm{C}\left\{{ }^{1} \mathrm{H}\right\}$ NMR $\left(101 \mathrm{MHz}, \mathrm{CDCl}_{3}\right) \delta=162.6\left(1 \mathrm{C}, \mathrm{C}^{5}\right), 148.8\left(2 \mathrm{C}, \mathrm{C}^{2}\right), 134.2\left(1 \mathrm{C}, \mathrm{C}^{4}\right)$, $129.5\left(3 C, C^{3}, C^{9}\right), 126.6\left(1 C, C^{10}\right), 122.4\left(1 C, C^{1}\right), 66.7\left(1 C, C^{6}\right), 29.0\left(1 C, C^{8}\right)$, $28.4\left(1 \mathrm{C}, \mathrm{C}^{7}\right), 18.0\left(1 \mathrm{C}, \mathrm{C}^{11}\right) \mathrm{ppm}$;

IR (ATR) $\tilde{v}=3097(\mathrm{~m}), 1723(\mathrm{~s}), 1628(\mathrm{~m}), 1595(\mathrm{w}), 1539(\mathrm{~s}), 1461(\mathrm{w}), 1459(\mathrm{w})$, $1341(\mathrm{~s}), 1278(\mathrm{~s}), 1168(\mathrm{~s}), 1079(\mathrm{w}), 1023(\mathrm{w}), 959(\mathrm{~s}), 919(\mathrm{~m}), 855(\mathrm{w}), 825(\mathrm{w})$, 777 (w), $719(\mathrm{~s}) \mathrm{cm}^{-1}$; 
HRMS (ESI) $m / z$ [M+Ag] $]^{+}$, calcd. for $\mathrm{C}_{13} \mathrm{H}_{14} \mathrm{~N}_{2} \mathrm{O}_{6} \mathrm{Ag}^{+}: 400.9897$, found 400.9903 .

\section{(Z)-Hex-4-en-1-yl 3,5-dinitrobenzoate (S5)}

According General Procedure $\mathbf{A}(\mathrm{Z})$-hex-4-en-1-ol $(0.47 \mathrm{~mL}$,
$4.0 \mathrm{mmol}, 1.0$ eq.) and 3,5 -dinitrobenzoyl chloride $(1.8 \mathrm{~g}$, Then $\mathrm{NEt}_{3}(1.1 \mathrm{~mL}, 8.0 \mathrm{mmol}, 2.0$ eq.) was added slowly and the reaction mixture was stirred for 5 days. The crude mixture was purified by column chromatography using cyclohexane:ethyl acetate 98:2 to afford (Z)-hex-4-en-1-yl 3,5-dinitrobenzoate (S5) as a yellow solid $(0.82 \mathrm{~g}, 2.8 \mathrm{mmol}, 69 \%)$.

Rf: ( $n$-pentane:diethyl ether 10:1) 0.34 ;

M.p. $26-28^{\circ} \mathrm{C}$;

${ }^{1} \mathrm{H} \mathrm{NMR}\left(400 \mathrm{MHz}, \mathrm{CDCl}_{3}\right) \delta=9.21\left(\mathrm{~m}, 1 \mathrm{H}, \mathrm{H}^{1}\right), 9.15\left(\mathrm{~m}, 2 \mathrm{H}, \mathrm{H}^{3}\right), 5.55\left(\mathrm{dq},{ }^{3} \mathrm{JHH}_{\mathrm{HH}}=\right.$ $\left.10.8,5.9 \mathrm{~Hz}, 1 \mathrm{H}, \mathrm{H}^{10}\right), 5.42\left(\mathrm{dtq},{ }^{3} \mathrm{JHH}_{\mathrm{HH}}=10.8,7.0 \mathrm{~Hz},{ }^{4} \mathrm{JHH}_{\mathrm{HH}}=1.8 \mathrm{~Hz}, 1 \mathrm{H}, \mathrm{H}^{9}\right), 4.46(\mathrm{t}$, $\left.{ }^{3} \mathrm{~J}_{\mathrm{HH}}=6.7 \mathrm{~Hz}, 2 \mathrm{H}, \mathrm{H}^{6}\right), 2.23\left(\mathrm{tdq},{ }^{3} \mathrm{JHH}_{\mathrm{HH}}=7.4,7.0 \mathrm{~Hz},{ }^{5} \mathrm{JHH}_{\mathrm{HH}}=0.9 \mathrm{~Hz}, 2 \mathrm{H}, \mathrm{H}^{8}\right), 1.91(\mathrm{tt}$, $\left.{ }^{3} \mathrm{~J}_{\mathrm{HH}}=7.4,6.7 \mathrm{~Hz}, 2 \mathrm{H}, \mathrm{H}^{7}\right), 1.63\left(\mathrm{ddt},{ }^{3} \mathrm{JHH}_{\mathrm{HH}}=5.9 \mathrm{~Hz},{ }^{4} \mathrm{JHH}_{\mathrm{HH}}=1.8 \mathrm{~Hz},{ }^{5} \mathrm{JHH}_{\mathrm{HH}}=0.9 \mathrm{~Hz}, 3 \mathrm{H}\right.$, $\left.\mathrm{H}^{11}\right) \mathrm{ppm}$;

${ }^{13} \mathrm{C}\left\{{ }^{1} \mathrm{H}\right\}$ NMR $\left(101 \mathrm{MHz}, \mathrm{CDCl}_{3}\right) \delta=162.6\left(1 \mathrm{C}, \mathrm{C}^{5}\right), 148.8\left(2 \mathrm{C}, \mathrm{C}^{2}\right), 134.2\left(1 \mathrm{C}, \mathrm{C}^{4}\right)$, $129.5\left(2 \mathrm{C}, \mathrm{C}^{3}\right), 128.7\left(1 \mathrm{C}, \mathrm{C}^{9}\right), 125.5\left(1 \mathrm{C}, \mathrm{C}^{10}\right), 122.4\left(1 \mathrm{C}, \mathrm{C}^{1}\right), 66.6\left(1 \mathrm{C}, \mathrm{C}^{6}\right)$, $28.4\left(1 \mathrm{C}, \mathrm{C}^{7}\right), 23.3\left(1 \mathrm{C}, \mathrm{C}^{8}\right), 12.9\left(1 \mathrm{C}, \mathrm{C}^{11}\right) \mathrm{ppm}$;

IR (ATR) $\tilde{v}=3098(\mathrm{~m}), 1842(\mathrm{w}), 1719(\mathrm{~s}), 1629(\mathrm{~m}), 1541(\mathrm{~s}), 1464(\mathrm{~m}), 1346(\mathrm{~s})$, $1284(\mathrm{~m}), 1171(\mathrm{~m}), 1075(\mathrm{~m}), 1022(\mathrm{~m}), 922(\mathrm{~m}), 882(\mathrm{~m}), 827(\mathrm{~m}), 773(\mathrm{~m}), 718(\mathrm{~s})$, $680(\mathrm{~m}) \mathrm{cm}^{-1}$;

HRMS (ESI) $m / z$ [M+Ag] $]^{+}$, calcd. for $\mathrm{C}_{13} \mathrm{H}_{14} \mathrm{~N}_{2} \mathrm{O}_{6} \mathrm{Ag}^{+}: 400.9897$, found 400.9902 . 


\section{Undec-10-en-1-yl acetate (S6)}

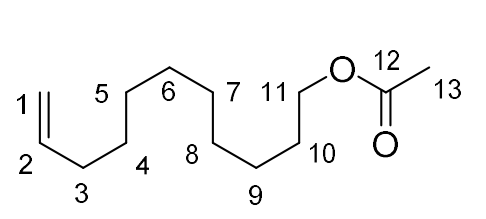

10-Undecen-1-ol $(1.00 \mathrm{~mL}, \quad 5.0 \mathrm{mmol}, \quad 1.0$ eq. $)$ and pyridine $(0.60 \mathrm{~mL}, 7.5 \mathrm{mmol}, 1.5$ eq. $)$ were diluted in $\mathrm{CH}_{2} \mathrm{Cl}_{2}(1 \mathrm{~mL})$. The mixture was cooled down to $0^{\circ} \mathrm{C}$ and acetyl chloride $(0.39 \mathrm{~mL}, 5.5 \mathrm{mmol}, 1.1 \mathrm{eq}$. $)$ was added dropwise. The reaction mixture was allowed to warm to ambient temperature and was stirred for $16 \mathrm{~h}$. Upon completion, the reaction mixture was diluted with $\mathrm{CH}_{2} \mathrm{Cl}_{2}$ and was washed with $1 \mathrm{~N} \mathrm{HCl}$, water, and brine. The organic layer was dried over $\mathrm{MgSO}_{4}$, filtered and the solvent was removed in vacuo. The crude mixture was purified by column chromatography using $n$-pentane: $\mathrm{CH}_{2} \mathrm{Cl}_{2}$ 1:1 to afford undec-10-en-1-yl acetate (S6) as a colourless oil $(0.99 \mathrm{~g}, 4.7 \mathrm{mmol}, 93 \%)$.

Rf: (n-pentane: $\mathrm{CH}_{2} \mathrm{Cl}_{2}$ 1:1) 0.48 ;

${ }^{1} \mathrm{H}$ NMR $\left(400 \mathrm{MHz}, \mathrm{CDCl}_{3}\right) \delta=5.80\left(\mathrm{ddt}, J_{\mathrm{HH}}=16.9,10.2,6.7 \mathrm{~Hz}, 1 \mathrm{H}, \mathrm{H}^{2}\right), 4.98(\mathrm{dq}$, $\left.J_{H H}=17.2,1.7 \mathrm{~Hz}, 1 \mathrm{H}, \mathrm{H}^{1 E}\right), 4.92\left(\mathrm{ddt}, J_{\mathrm{HH}}=10.2,2.4,1.2 \mathrm{~Hz}, 1 \mathrm{H}, \mathrm{H}^{1 \mathrm{z}}\right), 4.04\left(\mathrm{t}, J_{\mathrm{HH}}=\right.$ $\left.6.8 \mathrm{~Hz}, 2 \mathrm{H}, \mathrm{H}^{11}\right), 2.04\left(\mathrm{~s}, 3 \mathrm{H}, \mathrm{H}^{13}\right), 2.08-1.99\left(\mathrm{~m}, 2 \mathrm{H}, \mathrm{H}^{3}\right), 1.65-1.55\left(\mathrm{~m}, 2 \mathrm{H}, \mathrm{H}^{10}\right)$, $1.42-1.23\left(\mathrm{~m}, 12 \mathrm{H}, \mathrm{H}^{4}-\mathrm{H}^{9}\right) \mathrm{ppm}$;

HRMS (ESI) $m / z$ [M+Na] $]^{+}$calcd. for $\mathrm{C}_{13} \mathrm{H}_{24} \mathrm{NaO}_{2}{ }^{+}: 235.1669$, found: 235.1678 .

The analytic data are in agreement with the literature. ${ }^{5}$ 


\section{Undec-10-en-1-yl methacrylate (S7)}

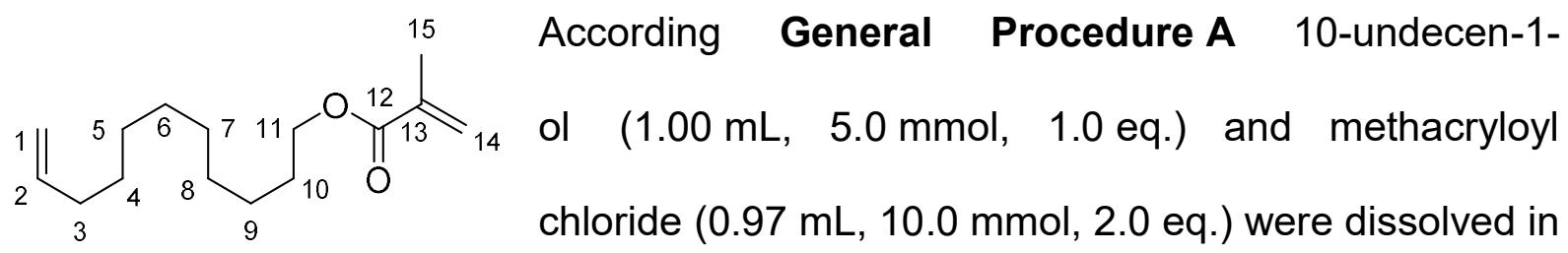

diethyl ether (5 mL). Then NEt3 $(1.4 \mathrm{~mL}, 10.0 \mathrm{mmol}, 2.0$ eq.) was added slowly and the reaction mixture was stirred for $24 \mathrm{~h}$. The crude mixture was purified by column chromatography using n-pentane: $\mathrm{CH}_{2} \mathrm{Cl}_{2}$ 3:1 to afford undec-10-en-1-yl methacrylate (S7) as a colourless oil (1.01 g, $4.2 \mathrm{mmol}, 85 \%)$.

Rf: (n-pentane: $\mathrm{CH}_{2} \mathrm{Cl}_{2}$ 3:1) 0.42;

${ }^{1} \mathrm{H}$ NMR $\left(400 \mathrm{MHz}, \mathrm{CDCl}_{3}\right) \delta=6.09(\mathrm{br} \mathrm{s}, 1 \mathrm{H}), 5.81\left(\mathrm{ddt}, \mathrm{J}_{\mathrm{HH}}=16.9,10.2,6.7 \mathrm{~Hz}, 1 \mathrm{H}\right)$, $5.54(\mathrm{~m}, 1 \mathrm{H}), 4.99(\mathrm{~m}, 1 \mathrm{H}), 4.93(\mathrm{~m}, 1 \mathrm{H}), 4.13\left(\mathrm{t}, \mathrm{J}_{\mathrm{HH}}=6.7 \mathrm{~Hz}, 2 \mathrm{H}\right), 2.04\left(\mathrm{q}, \mathrm{J}_{\mathrm{HH}}=6.9\right.$ $\mathrm{Hz}, 2 \mathrm{H}), 1.94\left(\mathrm{br} \mathrm{s}, \mathrm{J}_{\mathrm{HH}}=1.4 \mathrm{~Hz}, 3 \mathrm{H}\right), 1.66\left(\mathrm{p}, \mathrm{J}_{\mathrm{HH}}=6.7 \mathrm{~Hz}, 2 \mathrm{H}\right), 1.41-1.25(\mathrm{~m}, 12 \mathrm{H})$ ppm;

HRMS (ESI) $m / z$ [M+Na] ${ }^{+}$calcd. for $\mathrm{C}_{15} \mathrm{H}_{26} \mathrm{NaO}_{2}{ }^{+}:$261.1825, found: 261.1830.

The analytic data are in agreement with the literature. ${ }^{6}$

\section{2-(Pent-4-en-1-yl)isoindoline-1,3-dione (S8)}

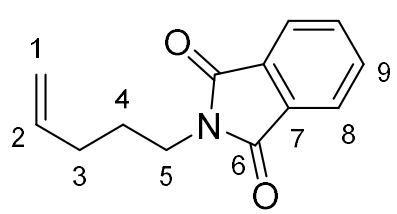

5-Bromopent-1-ene $(0.30 \mathrm{~mL}, 2.5 \mathrm{mmol}, 1.0$ eq. $)$ and potassium phthalimide (1.4 g, $7.5 \mathrm{mmol}, 3.0$ eq.) were suspended in DMF (20 mL). The reaction mixture was stirred at $60^{\circ} \mathrm{C}$ for $16 \mathrm{~h}$. The reaction mixture was diluted with ethyl acetate and washed with water (5x). The organic layer was dried over $\mathrm{MgSO}_{4}$, filtered and evaporated under vacuo. The crude mixture was purified by column chromatography using $n$ pentane:ethyl acetate 15:1 to afford 2-(pent-4-en-1-yl)isoindoline-1,3-dione (S8) as a colourless oil (441 mg, $2.0 \mathrm{mmol}, 82 \%)$. 
Rf: (n-pentane:ethyl acetate 15:1) 0.24 ;

${ }^{1} \mathrm{H}$ NMR $\left(300 \mathrm{MHz}, \mathrm{CDCl}_{3}\right) \delta=7.84\left(\mathrm{dd}, J_{\mathrm{HH}}=5.4,3.1 \mathrm{~Hz}, 2 \mathrm{H}\right), 7.71\left(\mathrm{dd}, J_{\mathrm{HH}}=5.5\right.$, $3.0 \mathrm{~Hz}, 2 \mathrm{H}$ ), $5.82\left(\mathrm{ddt}, J_{\mathrm{HH}}=16.8,10.2,6.5 \mathrm{~Hz}, 1 \mathrm{H}\right), 5.05\left(\mathrm{dq}, J_{\mathrm{HH}}=17.1,1.7 \mathrm{~Hz}, 1 \mathrm{H}\right)$, $4.98\left(\mathrm{dq}, \mathrm{JHH}_{\mathrm{HH}}=10.2,1.4 \mathrm{~Hz}, 1 \mathrm{H}\right), 3.70\left(\mathrm{t}, \mathrm{J}_{\mathrm{HH}}=7.3 \mathrm{~Hz}, 2 \mathrm{H}\right), 2.18-2.06(\mathrm{~m}, 2 \mathrm{H}), 1.87-$ $1.71(\mathrm{~m}, 2 \mathrm{H})$;

HRMS (ESI) $m / z$ [M+Na] ${ }^{+}$calcd. for $\mathrm{C}_{13} \mathrm{H}_{13} \mathrm{NNaO}_{2}{ }^{+}: 238.0838$, found: 238.0845 .

The analytic data are in agreement with the literature. ${ }^{5}$

\section{Undec-10-en-1-yl 4-methylbenzenesulfonate (S9)}

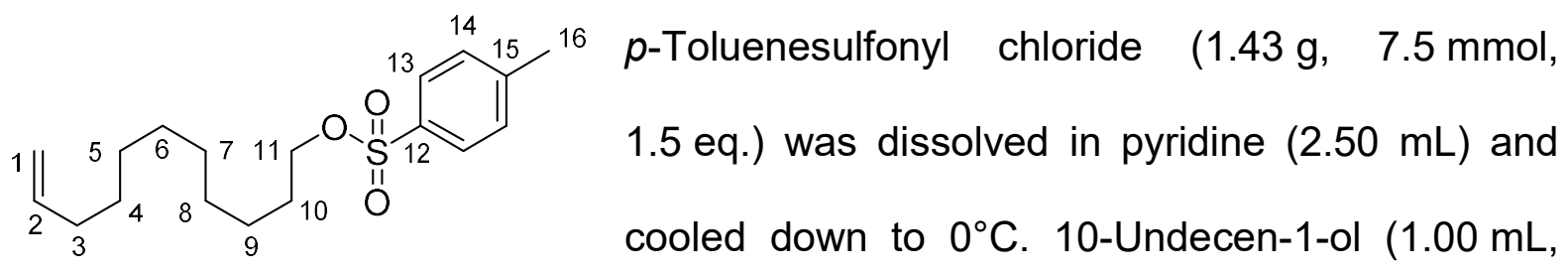

$5.0 \mathrm{mmol}, 1.0$ eq.) was added dropwise to the mixture which was then stirred for $2 \mathrm{~h}$. Upon completion, the reaction mixture was poured on a mixture ice/ $\mathrm{HCl}$ conc. and was extracted with ethyl acetate $(3 x)$. The combined organic layers were washed with water and brine, dried over $\mathrm{MgSO}_{4}$, filtered and evaporated under vacuo. The crude mixture was purified by column chromatography using a gradient $n$-pentane: $\mathrm{CH}_{2} \mathrm{Cl}_{2} 1: 9$ to $3: 7$ to afford undec-10-en-1-yl 4-methylbenzenesulfonate (S9) as a colourless oil (1.05 g, $3.2 \mathrm{mmol}, 65 \%)$.

Rf: (n-pentane: $\mathrm{CH}_{2} \mathrm{Cl}_{2}$ 9:1) 0.27 ;

${ }^{1} \mathrm{H}$ NMR $\left(400 \mathrm{MHz}, \mathrm{CDCl}_{3}\right) \delta=7.79(\mathrm{~m}, 2 \mathrm{H}), 7.34(\mathrm{~m}, 2 \mathrm{H}), 5.80\left(\mathrm{ddt}, J_{\mathrm{HH}}=17.0,10.2\right.$, $6.6 \mathrm{~Hz}, 1 \mathrm{H}), 4.99(\mathrm{~m}, 1 \mathrm{H}), 4.93(\mathrm{~m}, 1 \mathrm{H}), 4.02\left(\mathrm{t}, \mathrm{JHH}_{\mathrm{HH}}=6.5 \mathrm{~Hz}, 2 \mathrm{H}\right), 2.45(\mathrm{~s}, 3 \mathrm{H}), 2.03$ (m, 2H), $1.63(\mathrm{~m}, 2 \mathrm{H}), 1.40-1.31(\mathrm{~m}, 2 \mathrm{H}), 1.31-1.16(\mathrm{~m}, 10 \mathrm{H}) \mathrm{ppm}$;

HRMS (ESI) $m / z$ [M+Na] $]^{+}$calcd. for $\mathrm{C}_{18} \mathrm{H}_{28} \mathrm{NaO}_{3} \mathrm{~S}^{+}: 347.1651$, found: 347.1658 .

The analytic data are in agreement with the literature. ${ }^{5}$ 


\section{Methyl undec-10-en-1-yl sulfate (S10)}

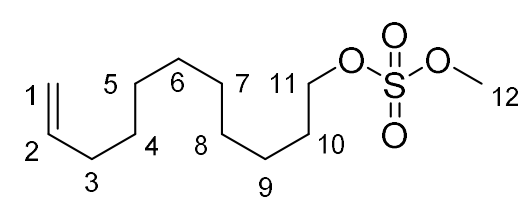

This compound was prepared according to a modified procedure from Ooi et al. ${ }^{7}$ Sulfur trioxide pyridine complex (0.56 g, $3.5 \mathrm{mmol}, 3.5 \mathrm{eq}$.) was suspended in tetrahydrofuran $(15 \mathrm{~mL}) .10$-Undecen-1-ol $(0.20 \mathrm{~mL}, 1.0 \mathrm{mmol}, 1.0$ eq. $)$ was added dropwise to the mixture which was then stirred at ambient temperature for $18 \mathrm{~h}$. The reaction mixture was diluted with ethyl acetate and washed with water (1x) and brine (2x). The organic layer was dried over $\mathrm{MgSO}_{4}$, filtered and evaporated under vacuo. The crude mixture $(0.20 \mathrm{~g})$ was suspended in $\mathrm{CH}_{2} \mathrm{Cl}_{2}(20 \mathrm{~mL})$. The round-bottom flask was wrapped in aluminium foil, proton-sponge $(1.07 \mathrm{~g}, 5.0 \mathrm{mmol}, 5.0 \mathrm{eq}$.) was added in one portion and trimethyloxonium tetrafluoroborate $(0.74 \mathrm{~g}, 5.0 \mathrm{mmol}, 5.0$ eq.) was added in two portions. The reaction mixture was stirred at ambient temperature for $5 \mathrm{~h}$. Upon completion, the reaction mixture was treated with $1 \mathrm{~N} \mathrm{HCl}$ and extracted with $\mathrm{CH}_{2} \mathrm{Cl}_{2}$ (3x). The combined organic layers were dried over $\mathrm{MgSO}_{4}$, filtered and the solvent was removed in vacuo. The crude mixture was purified by column chromatography using a gradient of $n$-pentane: $\mathrm{CH}_{2} \mathrm{Cl}_{2} 4: 1$ to $3: 2$ to afford methyl undec-10-en-1-yl sulfate (S10) as a colourless oil $(0.14 \mathrm{~g}, 0.5 \mathrm{mmol}, 54 \%$ over two steps).

Rf: (n-pentane: $\left.\mathrm{CH}_{2} \mathrm{Cl}_{2} 4: 1\right)$ 0.11;

${ }^{1} \mathrm{H}$ NMR $\left(400 \mathrm{MHz}, \mathrm{CDCl}_{3}\right) \delta=5.80\left(\mathrm{dddd},{ }^{3} \mathrm{JHH}_{\mathrm{HH}}=17.1,12.5,6.7,3.3 \mathrm{~Hz}, 1 \mathrm{H}, \mathrm{H}^{2}\right), 4.99$ (ddt, ${ }^{3} \mathrm{JHH}_{\mathrm{HH}}=17.1 \mathrm{~Hz},{ }^{2} \mathrm{~J}_{\mathrm{HH}}=2.4 \mathrm{~Hz},{ }^{4} \mathrm{~J}_{\mathrm{HH}}=1.6 \mathrm{~Hz}, 1 \mathrm{H}, \mathrm{H}^{1 \mathrm{z}}$ ), 4.93 (ddt, ${ }^{3} \mathrm{~J}_{\mathrm{HH}}=10.2 \mathrm{~Hz}$, $\left.{ }^{2} J_{\mathrm{HH}}=2.5 \mathrm{~Hz},{ }^{4} \mathrm{~J}_{\mathrm{HH}}=1.3 \mathrm{~Hz}, 1 \mathrm{H}, \mathrm{H}^{1 E}\right), 4.26\left(\mathrm{t},{ }^{3} \mathrm{~J}_{\mathrm{HH}}=6.6 \mathrm{~Hz}, 2 \mathrm{H}, \mathrm{H}^{11}\right), 3.95(\mathrm{~s}, 3 \mathrm{H}$, $\left.\mathrm{H}^{12}\right), 2.04\left(\mathrm{qd},{ }^{3} \mathrm{JHH}_{\mathrm{HH}}=8.1,6.8 \mathrm{~Hz}, 2 \mathrm{H}, \mathrm{H}^{3}\right), 1.76\left(\mathrm{p},{ }^{3} \mathrm{JHH}_{\mathrm{HH}}=6.7 \mathrm{~Hz}, 2 \mathrm{H}, \mathrm{H}^{10}\right), 1.45-1.25$ $\left(\mathrm{m}, 12 \mathrm{H}, \mathrm{H}^{4-9}\right) \mathrm{ppm}$; 
${ }^{13} \mathrm{C}\left\{{ }^{1} \mathrm{H}\right\}$ NMR $\left(101 \mathrm{MHz}, \mathrm{CDCl}_{3}\right) \delta=139.3\left(1 \mathrm{C}, \mathrm{C}^{2}\right), 114.3\left(1 \mathrm{C}, \mathrm{C}^{1}\right), 73.8\left(1 \mathrm{C}, \mathrm{C}^{11}\right)$, $58.4\left(1 \mathrm{C}, \mathrm{C}^{12}\right), 33.9$ (1C, C $\left.\mathrm{C}^{3}\right), 29.5(2 \mathrm{C}), 29.2(1 \mathrm{C}), 29.1$ (1C), 29.0 (1C, C4), 28.9 (1C), $25.5\left(1 \mathrm{C}, \mathrm{C}^{9}\right) \mathrm{ppm}$;

IR (ATR) $\tilde{v}=2926(\mathrm{~m}), 2855(\mathrm{~m}), 1641(\mathrm{w}), 1463(\mathrm{w}), 1397(\mathrm{~m}), 1194(\mathrm{~s}), 997(\mathrm{~m}), 946$ (m), $914(m), 826(m), 799(m), 772(m), 723(w) \mathrm{cm}^{-1}$;

HRMS (ESI) $m / z$ [M+Na] $]^{+}$calcd. for $\mathrm{C}_{12} \mathrm{H}_{24} \mathrm{NaO}_{4} \mathrm{~S}^{+}: 287.1288$, found: 287.1291 .

\section{Diphenyl undec-10-en-1-yl phosphate (S11)}

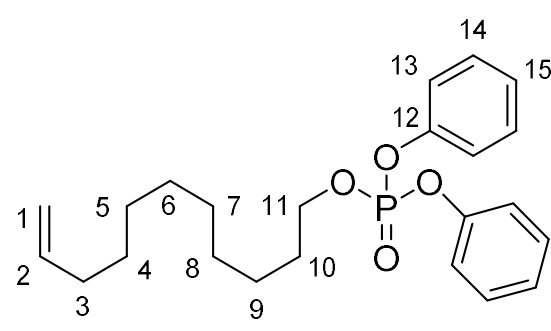

This compound was prepared according to a modified procedure from Dieter et al. ${ }^{8}$ 10-Undecen-1-ol $\left(0.20 \mathrm{~mL}, 1.0 \mathrm{mmol}, 1.0\right.$ eq.) and $\mathrm{NEt}_{3}(0.28 \mathrm{~mL}$, $2.0 \mathrm{mmol}, 2.0$ eq.) were diluted in dry tetrahydrofuran

$(5 \mathrm{~mL})$ and the resulting solution was cooled to $0^{\circ} \mathrm{C}$. Diphenyl phosphorochloridate (0.31 mL, $1.5 \mathrm{mmol}, 1.5$ eq.) was added dropwise into the reaction mixture which was allowed to warm to ambient temperature and was stirred for $24 \mathrm{~h}$. The reaction mixture was diluted with ethyl acetate and water, and the aqueous layer was extracted with ethyl acetate (3x). The combined organic layers were washed with water and brine, dried over $\mathrm{MgSO}_{4}$, filtered and evaporated under vacuo. The crude mixture was purified by column chromatography using $n$-pentane: $\mathrm{CH}_{2} \mathrm{Cl}_{2} 1: 1$ to afford diphenyl undec-10-en-1-yl phosphate (S11) as a light yellow oil $(0.27 \mathrm{~g}, 0.7 \mathrm{mmol}, 68 \%)$.

Rf: $\left(\mathrm{CH}_{2} \mathrm{Cl}_{2} 100 \%\right) 0.54$

${ }^{1} \mathrm{H}$ NMR $\left(400 \mathrm{MHz}, \mathrm{CDCl}_{3}\right) \delta=7.34\left(\mathrm{~m}, 4 \mathrm{H}, \mathrm{H}^{14}\right), 7.21\left(\mathrm{~m}, 6 \mathrm{H}, \mathrm{H}^{13}, \mathrm{H}^{15}\right), 5.81$ (ddt, $\left.{ }^{3} J_{\mathrm{HH}}=16.8,9.9,6.7 \mathrm{~Hz}, 1 \mathrm{H}, \mathrm{H}^{2}\right), 4.99\left(\mathrm{dq},{ }^{3} \mathrm{JHH}_{\mathrm{HH}}=17.1 \mathrm{~Hz},{ }^{2} \mathrm{JHH}_{\mathrm{HH}}=1.8 \mathrm{~Hz}, 1 \mathrm{H}, \mathrm{H}^{1 E}\right), 4.93$ $\left(\mathrm{dd},{ }^{3} \mathrm{~J}_{\mathrm{HH}}=10.1 \mathrm{~Hz},{ }^{2} \mathrm{~J}_{\mathrm{HH}}=2.3 \mathrm{~Hz}, 1 \mathrm{H}, \mathrm{H}^{1 \mathrm{z}}\right), 4.24\left(\mathrm{td},{ }^{3} \mathrm{~J}_{\mathrm{PH}}=7.7 \mathrm{~Hz},{ }^{3} \mathrm{JHH}_{\mathrm{HH}}=6.9 \mathrm{~Hz}, 2 \mathrm{H}\right.$, 
$\left.\mathrm{H}^{11}\right), 2.04\left(\mathrm{q},{ }^{3} \mathrm{~J}_{\mathrm{HH}}=7.1 \mathrm{~Hz}, 2 \mathrm{H}, \mathrm{H}^{3}\right), 1.69\left(\mathrm{p},{ }^{3} \mathrm{JHH}_{\mathrm{HH}}=6.9 \mathrm{~Hz}, 2 \mathrm{H}, \mathrm{H}^{10}\right), 1.43-1.19(\mathrm{~m}$, $\left.12 \mathrm{H}, \mathrm{H}^{4-9}\right) \mathrm{ppm}$;

${ }^{13} \mathrm{C}\left\{{ }^{1} \mathrm{H}\right\}$ NMR $\left(101 \mathrm{MHz}, \mathrm{CDCl}_{3}\right) \delta=150.8\left(\mathrm{~d},{ }^{2} \mathrm{JPC}=7.2 \mathrm{~Hz}, 2 \mathrm{C}, \mathrm{C}^{12}\right), 139.3\left(1 \mathrm{C}, \mathrm{C}^{2}\right)$, $129.9\left(4 \mathrm{C}, \mathrm{C}^{14}\right), 125.4\left(2 \mathrm{C}, \mathrm{C}^{15}\right), 120.2\left(\mathrm{~d},{ }^{3} \mathrm{JPC}=4.9 \mathrm{~Hz}, 4 \mathrm{C}, \mathrm{C}^{13}\right), 114.3\left(1 \mathrm{C}, \mathrm{C}^{1}\right), 69.6$ $\left(\mathrm{d},{ }^{2} \mathrm{JPC}_{\mathrm{PC}}=6.6 \mathrm{~Hz}, 1 \mathrm{C}, \mathrm{C}^{11}\right), 33.9\left(1 \mathrm{C}, \mathrm{C}^{3}\right), 30.3\left(\mathrm{~d},{ }^{3} \mathrm{JPC}_{\mathrm{PC}}=6.8 \mathrm{~Hz}, 1 \mathrm{C}, \mathrm{C}^{10}\right), 29.5(2 \mathrm{C})$, 29.2 (1C), $29.1(1 \mathrm{C}), 29.0\left(1 \mathrm{C}, \mathrm{C}^{4}\right), 25.4\left(1 \mathrm{C}, \mathrm{C}^{9}\right) \mathrm{ppm}$;

${ }^{31} \mathrm{P}$ NMR $\left(162 \mathrm{MHz}, \mathrm{CDCl}_{3}\right) \delta=-11.81\left(\mathrm{t},{ }^{3} \mathrm{JPH}=7.7 \mathrm{~Hz}\right) \mathrm{ppm}$;

IR (ATR) $\tilde{v}=3075(\mathrm{w}), 2926(\mathrm{~m}), 2855(\mathrm{w}), 1640(\mathrm{w}), 1591(\mathrm{~m}), 1489(\mathrm{~m}), 1457(\mathrm{w})$, $1394(w), 1287(m), 1221(m), 1189(s), 1163(m), 1023(m), 1008(m), 940(s), 904$ $(\mathrm{m}), 823(\mathrm{w}), 753(\mathrm{~m}), 687(\mathrm{~s}) \mathrm{cm}^{-1}$;

HRMS (ESI) $m / z$ [M+Na] $]^{+}$calcd. for $\mathrm{C}_{23} \mathrm{H}_{31} \mathrm{NaO}_{4} \mathrm{P}^{+}:$425.1852, found: 425.1845 .

\section{1-(Allyloxy)-4-nitrobenzene (S12)}

$\mathrm{O}_{2} \mathrm{~N} 1$ This compound was prepared according to a modified procedure from Jana et al. ${ }^{9}$ 4-Nitrophenol $(1.0 \mathrm{~g}, 7.2 \mathrm{mmol}$, 1.0 eq.) and $\mathrm{K}_{2} \mathrm{CO}_{3}(2.0 \mathrm{~g}, 14.4 \mathrm{mmol}, 2.0$ eq.) were combined in $\mathrm{DMF}(15 \mathrm{~mL})$. To this suspension was added allyl bromide $(623 \mu \mathrm{L}, 7.2 \mathrm{mmol}, 1.0 \mathrm{eq}$.$) and the mixture was$ stirred at ambient temperature for $16 \mathrm{~h}$. The reaction mixture was diluted with ethyl acetate, washed with a saturated solution of $\mathrm{NH}_{4} \mathrm{Cl}(1 \mathrm{x})$ as well as with water (5x). The resulting organic layer was dried over $\mathrm{MgSO}_{4}$, filtered and evaporated under vacuo. The crude mixture was purified by column chromatography using cyclohexane:ethyl acetate 4:1 to afford 1-(allyloxy)-4-nitrobenzene (S12) as a yellow oil $(1.1 \mathrm{~g}, 6.1 \mathrm{mmol}$, $84 \%)$.

Rf: (cyclohexane:ethyl acetate 4:1) 0.55; 
${ }^{1} \mathrm{H}$ NMR $\left(400 \mathrm{MHz}, \mathrm{CDCl}_{3}\right) \delta=8.19(\mathrm{~m}, 2 \mathrm{H}), 6.97(\mathrm{~m}, 2 \mathrm{H}), 6.04\left(\mathrm{ddt},{ }^{3} \mathrm{JHH}_{\mathrm{HH}}=17.3,10.6\right.$, $\left.5.3 \mathrm{~Hz}, 1 \mathrm{H}, \mathrm{H}^{6}\right), 5.43\left(\mathrm{dq},{ }^{3} \mathrm{~J}_{\mathrm{HH}}=17.2,{ }^{4} \mathrm{~J}_{\mathrm{HH}}=1.5 \mathrm{~Hz}, 1 \mathrm{H}, \mathrm{H}^{7}\right), 5.35\left(\mathrm{dq},{ }^{3} \mathrm{~J}_{\mathrm{HH}}=10.6\right.$, $\left.{ }^{4} \mathrm{~J}_{\mathrm{HH}}=1.4 \mathrm{~Hz}, 1 \mathrm{H} ; \mathrm{H}^{7 \mathrm{z}}\right), 4.63\left(\mathrm{dt},{ }^{3} \mathrm{JHH}_{\mathrm{HH}}=5.3 \mathrm{~Hz},{ }^{4} \mathrm{~J}_{\mathrm{HH}}=1.5 \mathrm{~Hz}, 2 \mathrm{H}, \mathrm{H}^{5}\right) \mathrm{ppm}$;

HRMS (ESI) $\mathrm{m} / \mathrm{z}[\mathrm{M}+\mathrm{Na}]^{+}$calcd. for $\mathrm{C}_{9} \mathrm{H}_{9} \mathrm{NNaO}_{3}{ }^{+}: 202.0475$, found: 202.0477 .

The analytic data are in agreement with the literature. ${ }^{10}$

\section{Allyl 4-methylbenzenesulfonate (S13)}

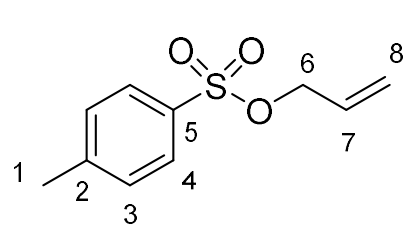

$\mathrm{NaH}$ (480 mg, $12 \mathrm{mmol}, 1.2$ eq., 60\% in mineral oil) was suspended in diethyl ether and cooled down to $0^{\circ} \mathrm{C}$. Allyl alcohol (680 $\mu \mathrm{L}, 10 \mathrm{mmol}, 1.0$ eq.) was added and the mixture was stirred at $0^{\circ} \mathrm{C}$ for 30 min. Finally, a solution of $p$-toluenesulfonyl chloride $(1.91 \mathrm{~g}$, $10 \mathrm{mmol}, 1.0$ eq.) in diethyl ether $(20 \mathrm{~mL})$ was added dropwise to the mixture which was then stirred for $3 \mathrm{~h}$. Upon completion, the reaction mixture was diluted with diethyl ether, washed with a saturated solution of $\mathrm{NH}_{4} \mathrm{Cl}$, water and brine, dried over $\mathrm{MgSO}_{4}$, filtered and evaporated under vacuo. The crude mixture was purified by column chromatography using $n$-pentane:ethyl acetate $95: 5$ to afford allyl 4methylbenzenesulfonate (S13) as a colourless oil (1.62 g, $7.6 \mathrm{mmol}, 76 \%)$.

Rf: ( $n$-pentane:ethyl acetate 9:1) 0.37 ;

${ }^{1} \mathrm{H}$ NMR $\left(300 \mathrm{MHz}, \mathrm{CDCl}_{3}\right) \delta=7.78(\mathrm{~m}, 2 \mathrm{H}), 7.34(\mathrm{~m}, 2 \mathrm{H}), 5.80\left(\mathrm{ddt}, \mathrm{JHH}_{\mathrm{HH}}=17.1,10.3\right.$, $\left.5.9 \mathrm{~Hz}, 1 \mathrm{H}, \mathrm{H}^{7}\right), 5.31\left(\mathrm{dq}, \mathrm{J}_{\mathrm{HH}}=17.1,1.4 \mathrm{~Hz}, 1 \mathrm{H}, \mathrm{H}^{8 E}\right), 5.24\left(\mathrm{dq}, \mathrm{J}_{\mathrm{HH}}=10.3,1.2 \mathrm{~Hz}\right.$, $\left.1 \mathrm{H}, \mathrm{H}^{8 \mathrm{Z}}\right), 4.51\left(\mathrm{dt}, \mathrm{J}_{\mathrm{HH}}=5.9,1.3 \mathrm{~Hz}, 2 \mathrm{H}, \mathrm{H}^{6}\right), 2.44\left(\mathrm{~s}, 3 \mathrm{H}, \mathrm{H}^{1}\right) \mathrm{ppm}$;

MS (EI) m/z (\%) = $212.0(5)[\mathrm{M}]^{+}, 155.0(46)\left[\mathrm{M}-\mathrm{C}_{3} \mathrm{H}_{5} \mathrm{O}\right]^{+}, 91.1(100)\left[\mathrm{M}-\mathrm{C}_{3} \mathrm{H}_{5} \mathrm{O}{ }_{3} \mathrm{~S}\right]^{+}$.

The analytic data are in agreement with the literature. ${ }^{11}$ 


\section{N,N-diallyl-4-methylbenzenesulfonamide (S14)}

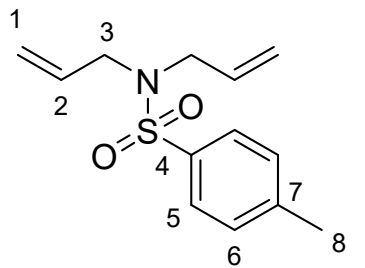

This compound was prepared according to a modified procedure from Hayashi et al. ${ }^{12} p$-Toluenesulfonyl chloride $(1.91 \mathrm{~g}$, $10 \mathrm{mmol}, 1.0$ eq.) was dissolved in $\mathrm{CH}_{2} \mathrm{Cl}_{2}(35 \mathrm{~mL})$. To this solution was added diallylamine $\left(1.23 \mathrm{~mL}, 10 \mathrm{mmol}, 1.0\right.$ eq.) and $\mathrm{NEt}_{3}(1.53 \mathrm{~mL}$, $11 \mathrm{mmol}, 1.1$ eq.) and the mixture was stirred at ambient temperature for $4 \mathrm{~h}$. Upon completion, the reaction mixture was poured on a saturated solution of $\mathrm{NH}_{4} \mathrm{Cl}$ and was extracted with $\mathrm{CH}_{2} \mathrm{Cl}_{2}$. The organic layer was washed with water and brine, dried over $\mathrm{MgSO}_{4}$, filtered and evaporated under vacuo. The crude mixture was purified by column chromatography using $n$-pentane:ethyl acetate $9: 1$ to afford $N, N$-diallyl-4methylbenzenesulfonamide (S14) as a colourless oil $(2.34 \mathrm{~g}, 9.3 \mathrm{mmol}, 93 \%)$.

Rf: (n-pentane:ethyl acetate 9:1) 0.48 ;

${ }^{1} \mathrm{H}$ NMR $\left(300 \mathrm{MHz}, \mathrm{CDCl}_{3}\right) \delta=7.71(\mathrm{~m}, 2 \mathrm{H}), 7.29(\mathrm{~m}, 2 \mathrm{H}), 5.60\left(\mathrm{ddt}, \mathrm{J}_{\mathrm{HH}}=17.5,9.8\right.$, $\left.6.4 \mathrm{~Hz}, 2 \mathrm{H}, \mathrm{H}^{2}\right), 5.16\left(\mathrm{~m}, 2 \mathrm{H}, \mathrm{H}^{1 \mathrm{a}}\right), 5.11\left(\mathrm{~m}, 2 \mathrm{H}, \mathrm{H}^{1 \mathrm{~b}}\right), 3.80\left(\mathrm{dt}, \mathrm{J}_{\mathrm{HH}}=6.2,1.3 \mathrm{~Hz}, 4 \mathrm{H}\right.$, $\left.\mathrm{H}^{3}\right), 2.42\left(\mathrm{~s}, 3 \mathrm{H}, \mathrm{H}^{8}\right) \mathrm{ppm}$;

HRMS (ESI) $m / z$ [M+Na] ${ }^{+}$calcd. for $\mathrm{C}_{13} \mathrm{H}_{17} \mathrm{NNaO}_{2} \mathrm{~S}^{+}: 274.0872$, found: 274.0878 .

The analytic data are in agreement with the literature. ${ }^{12}$ 


\section{Dichloro(p-tolyl)-- $\lambda^{3}$-iodane (S15) ${ }^{14}$}

$\mathrm{Cl}-\mathrm{I}-\mathrm{Cl}$ This compound was prepared according to a modified procedure from Tao et al. ${ }^{13}$ 4-lodotoluene $(1.0 \mathrm{~g}, 4.6 \mathrm{mmol}, 1.0 \mathrm{eq}$.$) was dissolved in$ acetonitrile $(4.6 \mathrm{~mL})$. water $(3.5 \mathrm{~mL})$ and aq. $\mathrm{NaOCl}(10 \%, 3.5 \mathrm{~mL})$ were added slowly to the solution. Dropwise addition of aq. $\mathrm{HCl}(37 \%, 2.3 \mathrm{~mL})$ led to a yellow precipitate. The residue was filtered and washed with water and $n$-pentane. Dichloro( $p$ tolyl)- $\lambda^{3}$-iodane (S15) was obtained as a yellow solid $(1.2 \mathrm{~g}, 4.1 \mathrm{mmol}, 89 \%)$. M.p. $102-105^{\circ} \mathrm{C}$;

${ }^{1} \mathrm{H}$ NMR $\left(400 \mathrm{MHz}, \mathrm{CDCl}_{3}\right) \delta=8.05\left(\mathrm{~m}, 2 \mathrm{H}, \mathrm{H}^{4}\right), 7.27\left(\mathrm{~m}, 2 \mathrm{H}, \mathrm{H}^{3}\right), 2.47(\mathrm{~s}, 3 \mathrm{H}$, $\left.\mathrm{H}^{1}\right) \mathrm{ppm}$;

${ }^{13} \mathrm{C}$ NMR $\left(101 \mathrm{MHz}, \mathrm{CDCl}_{3}\right) \quad \delta=143.4\left(1 \mathrm{C}, \mathrm{C}^{2}\right), 134.0\left(2 \mathrm{C}, \mathrm{C}^{4}\right), 132.5\left(2 \mathrm{C}, \mathrm{C}^{3}\right)$, $122.1\left(1 \mathrm{C}, \mathrm{C}^{5}\right), 21.6\left(1 \mathrm{C}, \mathrm{C}^{1}\right) \mathrm{ppm}$;

IR (ATR) $\tilde{v}=3774(\mathrm{w}), 3327(\mathrm{~s}), 2942(\mathrm{w}), 2808(\mathrm{~m}), 1598(\mathrm{~s}), 1484(\mathrm{~m}), 1481(\mathrm{~m})$, $1467(\mathrm{~m}), 1389(\mathrm{w}), 1299(\mathrm{w}), 1207(\mathrm{~m}), 1179(\mathrm{~m}), 1006(\mathrm{~s}), 994(\mathrm{~m}), 794(\mathrm{~m}) \mathrm{cm}^{-1}$;

MS (EI) m/z: $287.9(2)[\mathrm{M}]^{+}, 218.0(100)\left[\mathrm{M}-\mathrm{Cl}_{2}\right]^{+}, 91.0(54)\left[\mathrm{M}-\mathrm{ICl}_{2}\right]$.

The analytic data are in agreement with the literature. ${ }^{14}$ 


\section{General procedure B for the catalytic dichlorination of terminal alkenes}

The corresponding alkene $(0.20 \mathrm{mmol}, 1.0$ eq. $), p$-iodotoluene $(8.7 \mathrm{mg}, 0.04 \mathrm{mmol}$, 0.2 eq.), cesium chloride (101 mg, $0.60 \mathrm{mmol}, 3.0$ eq.) and Selectfluor ${ }^{\circledR}(78.0 \mathrm{mg}$, $0.22 \mathrm{mmol}, 1.1$ eq.) were dissolved in a solution of HFIP (302 mg, $1.80 \mathrm{mmol}, 9.0$ eq.) in $\mathrm{CH}_{2} \mathrm{Cl}_{2}(0.9 \mathrm{~mL})$. The mixture was cooled and stirred at $0^{\circ} \mathrm{C}$ (ice bath). After $8 \mathrm{~h}$, the reaction mixture was quenched with a saturated solution of $\mathrm{Na}_{2} \mathrm{~S}_{2} \mathrm{O}_{3}$ and extracted with $\mathrm{CH}_{2} \mathrm{Cl}_{2}$ (3x). The combined organic phases were dried over $\mathrm{MgSO}_{4}$, filtered and the solvent was evaporated under vacuo. The residue was dissolved in $0.6 \mathrm{~mL}$ of $\mathrm{CDCl}_{3}$ containing $0.1 \mathrm{mmol}$ of DMF (stock solution) and analysed by ${ }^{1} \mathrm{H}$ NMR. The sample was then evaporated under vacuo and purified by column chromatography using the indicated solvent ratio.

\section{5,6-Dichlorohexyl benzoate (2)}

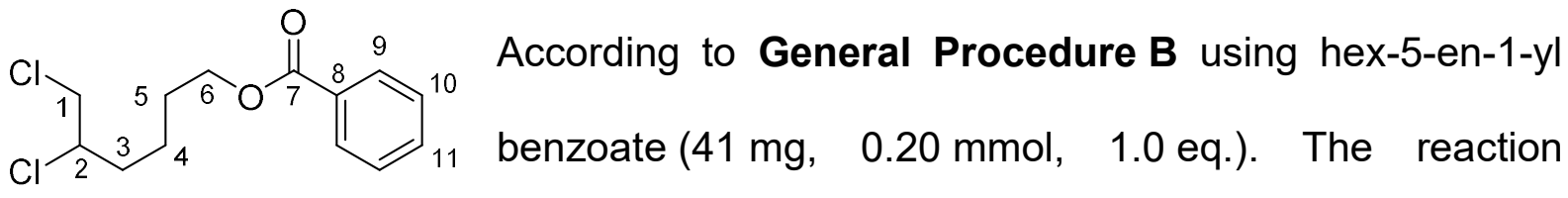
mixture was stirred at $0^{\circ} \mathrm{C}$ for $8 \mathrm{~h}$. The crude mixture was purified by column chromatography using cyclohexane:ethyl acetate $95: 5$ to afford 5,6-dichlorohexyl benzoate (2) as a colourless oil (41 $\mathrm{mg}, 0.15 \mathrm{mmol}, 75 \%)$.

For $1 \mathrm{mmol}$ scale, according to General Procedure B: hex-5-en-1-yl benzoate (204 mg, $1.0 \mathrm{mmol}, 1.0$ eq.), p-iodotoluene (44 mg, $0.2 \mathrm{mmol}, 0.2$ eq.), cesium chloride (505 mg, $3.0 \mathrm{mmol}, 3.0$ eq.) and Selectfluor ${ }^{\circledR}(390 \mathrm{mg}, 1.1 \mathrm{mmol}, 1.1$ eq.) were dissolved in a solution of HFIP (1.51 g, $9.0 \mathrm{mmol}, 9.0$ eq.) in $\mathrm{CH}_{2} \mathrm{Cl}_{2}(4.5 \mathrm{~mL})$. The reaction mixture was stirred at $0^{\circ} \mathrm{C}$ for $8 \mathrm{~h}$. The crude mixture was purified by column chromatography using cyclohexane:ethyl acetate $98: 2$ to afford 5,6-dichlorohexyl benzoate as a colourless oil $(213 \mathrm{mg}, 0.77 \mathrm{mmol}, 77 \%)$. 
Rf: (cyclohexane:ethyl acetate 95:5) 0.28;

${ }^{1} \mathrm{H}$ NMR $\left(500 \mathrm{MHz}, \mathrm{CDCl}_{3}\right) \delta=8.05\left(\mathrm{~m}, 2 \mathrm{H}, \mathrm{H}^{9}\right), 7.56\left(\mathrm{~m}, 1 \mathrm{H}, \mathrm{H}^{11}\right), 7.44\left(\mathrm{~m}, 2 \mathrm{H}, \mathrm{H}^{10}\right)$, $4.35\left(\mathrm{td},{ }^{3} \mathrm{~J}_{\mathrm{HH}}=6.4 \mathrm{~Hz}, \mathrm{~J}_{\mathrm{HH}}=1.7 \mathrm{~Hz}, 2 \mathrm{H} ; \mathrm{H}^{6}\right), 4.06$ (tdd, ${ }^{3} \mathrm{JHH}_{\mathrm{HH}}=8.8,5.0,3.5 \mathrm{~Hz}, 1 \mathrm{H}$, $\left.\mathrm{H}^{2}\right), 3.78\left(\mathrm{dd},{ }^{3} \mathrm{JHH}_{\mathrm{H}}=11.3,5.0 \mathrm{~Hz}, 1 \mathrm{H}, \mathrm{H}^{1 \mathrm{a}}\right), 3.66\left(\mathrm{dd},{ }^{3} \mathrm{JHH}_{\mathrm{HH}}=11.3,7.7 \mathrm{~Hz}, 1 \mathrm{H}, \mathrm{H}^{1 \mathrm{~b}}\right)$, $2.10\left(m, 1 H, H^{3 a}\right), 1.92-1.67\left(m, 4 H, H^{3 b}, H^{4 a}, H^{5}\right), 1.60\left(m, 1 H, H^{4 b}\right) p p m$;

${ }^{13} \mathrm{C}\left\{{ }^{1} \mathrm{H}\right\}$ NMR $\left(126 \mathrm{MHz}, \mathrm{CDCl}_{3}\right) \delta=166.7\left(1 \mathrm{C}, \mathrm{C}^{7}\right), 133.1\left(1 \mathrm{C}, \mathrm{C}^{11}\right), 130.5\left(1 \mathrm{C}, \mathrm{C}^{8}\right)$, $129.7\left(2 \mathrm{C}, \mathrm{C}^{9}\right), 128.5\left(2 \mathrm{C}, \mathrm{C}^{10}\right), 64.7\left(1 \mathrm{C}, \mathrm{C}^{6}\right), 60.9\left(1 \mathrm{C}, \mathrm{C}^{2}\right), 48.2\left(1 \mathrm{C}, \mathrm{C}^{1}\right), 34.8(1 \mathrm{C}$, $\left.\mathrm{C}^{3}\right), 28.3\left(1 \mathrm{C}, \mathrm{C}^{5}\right), 22.7\left(1 \mathrm{C}, \mathrm{C}^{4}\right) \mathrm{ppm}$;

IR (ATR) $\tilde{v}=2954(\mathrm{w}), 2870(\mathrm{w}), 1714(\mathrm{~s}), 1602(\mathrm{w}), 1585(\mathrm{w}), 1492(\mathrm{w}), 1451(\mathrm{~m})$, $1386(\mathrm{w}), 1314(\mathrm{~m}), 1270(\mathrm{~s}), 1219(\mathrm{~m}), 1193(\mathrm{~m}), 1176(\mathrm{~m}), 1114(\mathrm{~m}), 1070(\mathrm{~m}), 1026$ (m), $971(w), 936(w), 900(w), 806(w), 708(s), 687(m), 675(m), 661(m) \mathrm{cm}^{-1}$;

HRMS (ESI) $m / z$ [M+Na] $]^{+}$calcd. for $\mathrm{C}_{13} \mathrm{H}_{16} \mathrm{Cl}_{2} \mathrm{NaO}_{2}{ }^{+}: 297.0420$, found: 297.0420 .

\section{0,11-Dichloroundecyl acetate (3)}

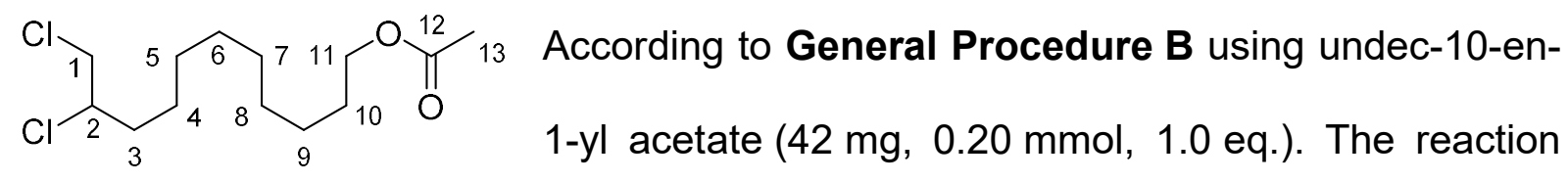
mixture was stirred at $0^{\circ} \mathrm{C}$ for $8 \mathrm{~h}$. The crude mixture was purified by column chromatography using cyclohexane:ethyl acetate $98: 2$ to afford 10,11-dichloroundecyl acetate (3) as a colourless oil ( $38 \mathrm{mg}, 0.13 \mathrm{mmol}, 66 \%$ ).

Rf: (n-pentane: $\mathrm{CH}_{2} \mathrm{Cl}_{2}$ 1:1) 0.45 ;

${ }^{1} \mathrm{H} \mathrm{NMR}\left(400 \mathrm{MHz}, \mathrm{CDCl}_{3}\right) \delta=4.04\left(\mathrm{t},{ }^{3} \mathrm{JHH}_{\mathrm{HH}}=6.8 \mathrm{~Hz}, 2 \mathrm{H}, \mathrm{H}^{11}\right), 4.06-3.98\left(\mathrm{~m}, 1 \mathrm{H}, \mathrm{H}^{2}\right)$, $3.75\left(\mathrm{dd},{ }^{2} \mathrm{~J}_{\mathrm{HH}}=11.3 \mathrm{~Hz},{ }^{3} \mathrm{JHH}_{\mathrm{HH}}=5.1 \mathrm{~Hz}, 1 \mathrm{H}, \mathrm{H}^{1 \mathrm{a}}\right), 3.64\left(\mathrm{dd},{ }^{2} \mathrm{~J}_{\mathrm{HH}}=11.3 \mathrm{~Hz},{ }^{3} \mathrm{JHH}_{\mathrm{HH}}=7.4 \mathrm{~Hz}\right.$, $\left.1 \mathrm{H}, \mathrm{H}^{1 \mathrm{~b}}\right), 2.04\left(\mathrm{~s}, 3 \mathrm{H}, \mathrm{H}^{13}\right), 1.96\left(\mathrm{~m}, 1 \mathrm{H}, \mathrm{H}^{3 \mathrm{a}}\right), 1.71\left(\mathrm{~m}, 1 \mathrm{H}, \mathrm{H}^{3 \mathrm{~b}}\right), 1.61\left(\mathrm{p},{ }^{3} \mathrm{JHH}=6.9,2 \mathrm{H}\right.$, $\left.\mathrm{H}^{10}\right), 1.53\left(\mathrm{~m}, 1 \mathrm{H}, \mathrm{H}^{4 a}\right), 1.41\left(\mathrm{~m}, 1 \mathrm{H}, \mathrm{H}^{4 b}\right), 1.30\left(\mathrm{~m}, 10 \mathrm{H}, \mathrm{H}^{5-9}\right) \mathrm{ppm}$; 
${ }^{13} \mathrm{C}\left\{{ }^{1} \mathrm{H}\right\} \operatorname{NMR}\left(101 \mathrm{MHz}, \mathrm{CDCl}_{3}\right) \delta=171.4\left(1 \mathrm{C}, \mathrm{C}^{12}\right), 64.7\left(1 \mathrm{C}, \mathrm{C}^{11}\right), 61.4\left(1 \mathrm{C}, \mathrm{C}^{2}\right), 48.4$ (1C, C $\left.{ }^{1}\right), 35.2\left(1 \mathrm{C}, \mathrm{C}^{3}\right), 29.5$ (1C), 29.4 (1C), 29.3 (1C), 29.1 (1C, C $\left.\mathrm{C}^{5}\right), 28.7\left(1 \mathrm{C}, \mathrm{C}^{10}\right)$, $26.0\left(1 \mathrm{C}, \mathrm{C}^{9}\right), 25.9\left(1 \mathrm{C}, \mathrm{C}^{4}\right), 21.2\left(1 \mathrm{C}, \mathrm{C}^{13}\right) \mathrm{ppm}$;

IR (ATR) $\tilde{v}=2928(\mathrm{~m}), 2856(\mathrm{~m}), 1737(\mathrm{~s}), 1464(\mathrm{w}), 1435(\mathrm{w}), 1387(\mathrm{w}), 1365(\mathrm{w})$, $1235(\mathrm{~s}), 1036(\mathrm{~m}), 969(\mathrm{w}), 912(\mathrm{w}), 726(\mathrm{~m}), 662(\mathrm{~m}) \mathrm{cm}^{-1}$;

HRMS (ESI) $m / z$ [M+Na] $]^{+}$calcd. for $\mathrm{C}_{13} \mathrm{H}_{24} \mathrm{Cl}_{2} \mathrm{NaO}_{2}{ }^{+}:$305.1046, found: 305.1055 .

\section{0,11-Dichloroundecyl methacrylate (4)}

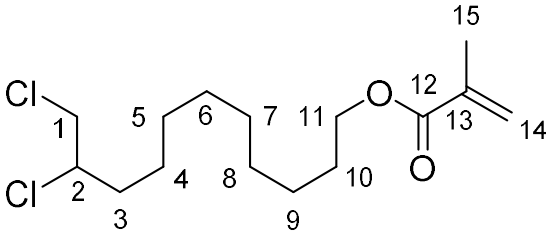

mixture was purified by column chromatography using a gradient $n$-pentane: $\mathrm{CH}_{2} \mathrm{Cl}_{2}$ 9:1 to $4: 1$ to afford 10,11-dichloroundecyl methacrylate (4) as a colourless oil (43 mg, $0.14 \mathrm{mmol}, 70 \%)$.

Rf: (n-pentane: $\mathrm{CH}_{2} \mathrm{Cl}_{2}$ 1:1) 0.48 ;

${ }^{1} \mathrm{H}$ NMR $\left(400 \mathrm{MHz}, \mathrm{CDCl}_{3}\right) \delta=6.09\left(\mathrm{~m}, 1 \mathrm{H}, \mathrm{H}^{14 \mathrm{a}}\right), 5.54\left(\mathrm{~m}, 1 \mathrm{H}, \mathrm{H}^{14 \mathrm{~b}}\right), 4.13\left(\mathrm{t},{ }^{3} \mathrm{JHH}_{\mathrm{HH}}=\right.$ $\left.6.7 \mathrm{~Hz}, 2 \mathrm{H}, \mathrm{H}^{11}\right), 4.03\left(\mathrm{dddd},{ }^{3}{ }_{\mathrm{HH}}=8.9,7.3,5.2,3.7 \mathrm{~Hz}, 1 \mathrm{H}, \mathrm{H}^{2}\right), 3.76\left(\mathrm{dd},{ }^{2}{ }_{\mathrm{HH}}=11.3\right.$ $\left.\mathrm{Hz},{ }^{3} \mathrm{JHH}_{\mathrm{HH}}=5.2 \mathrm{~Hz}, 1 \mathrm{H}, \mathrm{H}^{1 \mathrm{a}}\right), 3.65\left(\mathrm{dd},{ }^{2} \mathrm{~J}_{\mathrm{HH}}=11.2 \mathrm{~Hz},{ }^{3} \mathrm{~J}_{\mathrm{HH}}=7.4 \mathrm{~Hz}, 1 \mathrm{H}, \mathrm{H}^{1 \mathrm{~b}}\right), 1.98(\mathrm{~m}$, $\left.1 \mathrm{H}, \mathrm{H}^{3 a}\right), 1.94\left(\mathrm{~m}, 3 \mathrm{H}, \mathrm{H}^{15}\right), 1.76-1.61\left(\mathrm{~m}, 3 \mathrm{H}, \mathrm{H}^{3 \mathrm{~b}}, \mathrm{H}^{10}\right), 1.53\left(\mathrm{~m}, 1 \mathrm{H}, \mathrm{H}^{4 a}\right), 1.46-$ $1.22\left(\mathrm{~m}, 11 \mathrm{H}, \mathrm{H}^{4 \mathrm{~b}}, \mathrm{H}^{5-9}\right) \mathrm{ppm}$;

${ }^{13} \mathrm{C}\left\{{ }^{1} \mathrm{H}\right\} \operatorname{NMR}\left(101 \mathrm{MHz}, \mathrm{CDCl}_{3}\right) \delta=167.7\left(1 \mathrm{C}, \mathrm{C}^{12}\right), 136.7\left(1 \mathrm{C}, \mathrm{C}^{13}\right), 125.3\left(1 \mathrm{C}, \mathrm{C}^{14}\right)$, 64.9 (1C, $\left.\mathrm{C}^{11}\right), 61.4\left(1 \mathrm{C}, \mathrm{C}^{2}\right), 48.4\left(1 \mathrm{C}, \mathrm{C}^{1}\right), 35.2\left(1 \mathrm{C}, \mathrm{C}^{3}\right), 29.5$ (1C), $29.4(1 \mathrm{C}), 29.3$ (1C), $29.1\left(1 \mathrm{C}, \mathrm{C}^{5}\right), 28.7\left(1 \mathrm{C}, \mathrm{C}^{10}\right), 26.1\left(1 \mathrm{C}, \mathrm{C}^{9}\right), 25.9\left(1 \mathrm{C}, \mathrm{C}^{4}\right), 18.5\left(1 \mathrm{C}, \mathrm{C}^{15}\right) \mathrm{ppm}$; 
IR (ATR) $\tilde{v}=2928(\mathrm{~m}), 2856(\mathrm{w}), 1716(\mathrm{~s}), 1638(\mathrm{w}), 1455(\mathrm{w}), 1403(\mathrm{w}), 1376(\mathrm{w})$, $1321(\mathrm{~m}), 1296(\mathrm{~m}), 1161(\mathrm{~s}), 1012(\mathrm{w}), 939(\mathrm{~m}), 815(\mathrm{~m}), 726(\mathrm{~m}), 661(\mathrm{~m}) \mathrm{cm}^{-1}$;

HRMS (ESI) $m / z$ [M+Na] $]^{+}$calcd. for $\mathrm{C}_{15} \mathrm{H}_{26} \mathrm{Cl}_{2} \mathrm{NaO}_{2}{ }^{+}: 331.1202$, found: 331.1202 .

\section{0,11-Dichloroundecan-1-ol (5)}

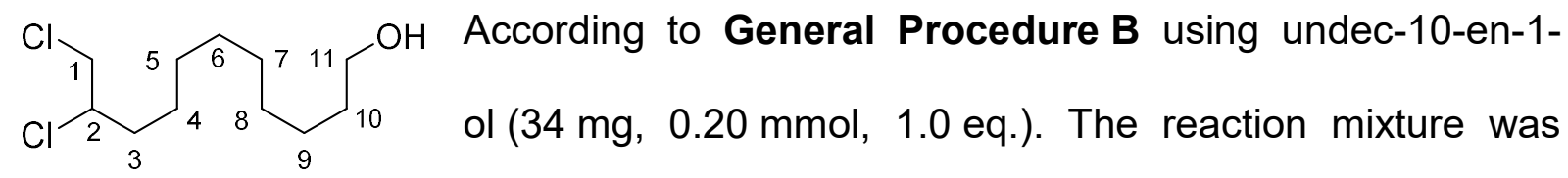

stirred at $0^{\circ} \mathrm{C}$ for $8 \mathrm{~h}$. The crude mixture was purified by column chromatography using $\mathrm{CH}_{2} \mathrm{Cl}_{2} 100 \%$ to afford 10,11-dichloroundecan-1-ol (5) as a colourless oil (36 mg, $0.15 \mathrm{mmol}, 75 \%)$.

Rf: $\left(\mathrm{CH}_{2} \mathrm{Cl}_{2} 100 \%\right) 0.24$;

${ }^{1} \mathrm{H}$ NMR $\left(400 \mathrm{MHz}, \mathrm{CDCl}_{3}, 299 \mathrm{~K}\right) \delta=4.02$ (dddd, $\mathrm{JHH}_{\mathrm{HH}}=9.0,7.3,5.2,3.8 \mathrm{~Hz}, 1 \mathrm{H}, \mathrm{H}^{2}$ ), $3.74\left(\mathrm{dd}, J_{\mathrm{HH}}=11.3,5.2 \mathrm{~Hz}, 1 \mathrm{H}, \mathrm{H}^{1 \mathrm{a}}\right), 3.66-3.59\left(\mathrm{~m}, 3 \mathrm{H}, \mathrm{H}^{1 \mathrm{~b}}, \mathrm{H}^{11}\right), 1.96$ (dddd, $J_{H H}=$ 14.2, 9.9, 5.7, 3.8 Hz, 1H, Ha), $1.69\left(\mathrm{dtd}, \mathrm{J}_{\mathrm{HH}}=14.2,9.4,4.6 \mathrm{~Hz}, 1 \mathrm{H}, \mathrm{H}^{3 \mathrm{~b}}\right), 1.59-1.50$ (m, 4H), $1.37-1.25(\mathrm{~m}, 10 \mathrm{H}) \mathrm{ppm}$;

HRMS (ESI) $m / z$ [M+Na] $]^{+}$calcd. for $\mathrm{C}_{11} \mathrm{H}_{22} \mathrm{Cl}_{2} \mathrm{NaO}^{+}:$263.0940, found: 263.0938 .

The analytic data are in agreement with the literature. ${ }^{15}$

\section{0,11-Dichloroundecanoic acid (6)}

作 stirred at $0^{\circ} \mathrm{C}$ for $8 \mathrm{~h}$. The crude mixture was purified by column chromatography using $n$-pentane:ethyl acetate 7:3 to afford 10,11-dichloroundecanoic acid (6) as a colourless oil (34 mg, $0.13 \mathrm{mmol}, 66 \%)$.

Rf: (cyclohexane:ethyl acetate 1:1) 0.44 ; 
${ }^{1} \mathrm{H}$ NMR $\left(300 \mathrm{MHz}, \mathrm{CDCl}_{3}\right) \delta=11.29(\mathrm{~s}, 1 \mathrm{H}, \mathrm{COOH}), 4.03$ (dddd, $\mathrm{JHH}_{\mathrm{H}}=8.9,7.3,5.1$, $3.8 \mathrm{~Hz}, 1 \mathrm{H}), 3.76\left(\mathrm{dd}, J_{\mathrm{HH}}=11.3,5.2 \mathrm{~Hz}, 1 \mathrm{H}\right), 3.65\left(\mathrm{dd}, J_{\mathrm{HH}}=11.3,7.4 \mathrm{~Hz}, 1 \mathrm{H}\right), 2.35$ $\left(\mathrm{t}, J_{H H}=7.5 \mathrm{~Hz}, 2 \mathrm{H}\right), 1.98$ (dddd, $\left.J_{H H}=14.1,9.9,5.6,3.7 \mathrm{~Hz}, 1 \mathrm{H}\right), 1.78-1.21(\mathrm{~m}, 13 \mathrm{H})$ ppm;

HRMS (ESI) $m / z[M+N a]^{+}$calcd. for $\mathrm{C}_{11} \mathrm{H}_{20} \mathrm{Cl}_{2} \mathrm{NaO}_{2}{ }^{+}: 277.0733$, found: 277.0703 .

The analytic data are in agreement with the literature. ${ }^{16}$

\section{1-Bromo-1,2-dichloroundecane (7)}

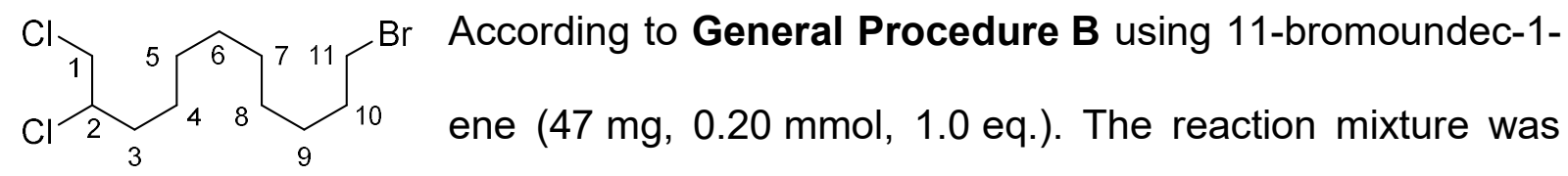
stirred at $0^{\circ} \mathrm{C}$ for $8 \mathrm{~h}$. The crude mixture was purified by column chromatography using $n$-pentane $100 \%$ to afford 11-bromo-1,2-dichloroundecane (7) as a colourless oil (43 mg, $0.14 \mathrm{mmol}, 71 \%$ ).

Rf: (cyclohexane 100\%) 0.45;

${ }^{1} \mathrm{H}$ NMR $\left(400 \mathrm{MHz}, \mathrm{CDCl}_{3}\right) \delta=4.03(\mathrm{~m}, 1 \mathrm{H}), 3.76\left(\mathrm{dd}, \mathrm{J}_{\mathrm{HH}}=11.3,5.2,1 \mathrm{H}\right), 3.65(\mathrm{dd}$, $\left.J_{H H}=11.2,7.4,1 \mathrm{H}\right), 3.41\left(\mathrm{t}, J_{\mathrm{HH}}=6.8,2 \mathrm{H}\right), 1.98(\mathrm{~m}, 1 \mathrm{H}), 1.85(\mathrm{~m}, 2 \mathrm{H}), 1.71(\mathrm{~m}, 1 \mathrm{H})$, $1.61-1.48(\mathrm{~m}, 1 \mathrm{H}), 1.48-1.26(\mathrm{~m}, 11 \mathrm{H}) \mathrm{ppm}$;

MS (EI) $m / z(\%)=302.0(52), 304.0$ (100), 306.1 (38), $308.0(12)[M]^{+}$, calcd. for $[M]^{+}$: 302.0 (62), 304.0 (100), 306.0 (46), 308.0 (8).

The analytic data are in agreement with the literature. ${ }^{15}$

\section{0,11-Dichloroundecyl methyl sulfate (8)}

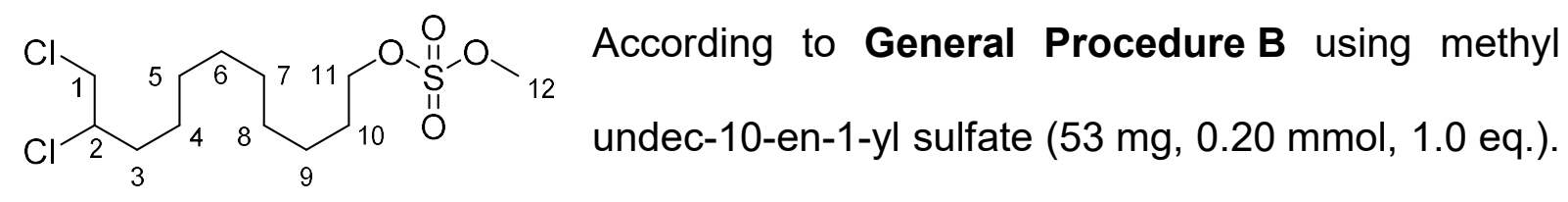

The reaction mixture was stirred at $0^{\circ} \mathrm{C}$ for $8 \mathrm{~h}$. The crude mixture was purified by S26 
column chromatography using cyclohexane:ethyl acetate 9:1 to afford 10,11dichloroundecyl methyl sulfate as a colourless oil ( $35 \mathrm{mg}, 0.10 \mathrm{mmol}, 52 \%)$.

Rf: (cyclohexane:ethyl acetate 9:1) 0.35;

${ }^{1} \mathrm{H} \mathrm{NMR}\left(599 \mathrm{MHz}, \mathrm{CDCl}_{3}\right) \delta=4.26\left(\mathrm{t},{ }^{3} \mathrm{JHH}_{\mathrm{HH}}=6.6 \mathrm{~Hz}, 2 \mathrm{H}, \mathrm{H}^{11}\right), 4.03\left(\mathrm{dddd},{ }^{3} \mathrm{JHH}_{\mathrm{HH}}=8.9\right.$, 7.3, 5.1, 3.7 Hz, $\left.1 \mathrm{H}, \mathrm{H}^{2}\right), 3.94\left(\mathrm{~s}, 3 \mathrm{H}, \mathrm{H}^{12}\right), 3.76\left(\mathrm{dd},{ }^{2} \mathrm{~J}_{\mathrm{HH}}=11.3 \mathrm{~Hz},{ }^{3} \mathrm{JHH}_{\mathrm{HH}}=5.2 \mathrm{~Hz}, 1 \mathrm{H}\right.$, $\left.\mathrm{H}^{1 \mathrm{a}}\right), 3.65\left(\mathrm{dd},{ }^{2} \mathrm{~J}_{\mathrm{HH}}=11.3 \mathrm{~Hz},{ }^{3} \mathrm{JHH}_{\mathrm{HH}}=7.4 \mathrm{~Hz}, 1 \mathrm{H}, \mathrm{H}^{1 \mathrm{~b}}\right), 1.98\left(\mathrm{dddd},{ }^{2} \mathrm{JHH}_{\mathrm{HH}}=14.2 \mathrm{~Hz}\right.$, $\left.{ }^{3} J_{H H}=10.3,5.8,3.8 \mathrm{~Hz}, 1 \mathrm{H}, \mathrm{H}^{3 a}\right), 1.76\left(\mathrm{~m}, 2 \mathrm{H}, \mathrm{H}^{10}\right), 1.70\left(\mathrm{~m}, 1 \mathrm{H}, \mathrm{H}^{3 b}\right), 1.55(\mathrm{~m}, 1 \mathrm{H}$, $\left.\mathrm{H}^{4 a}\right), 1.41\left(\mathrm{~m}, 3 \mathrm{H}, \mathrm{H}^{4 b}, \mathrm{H}^{9}\right), 1.36-1.26\left(\mathrm{~m}, 8 \mathrm{H}, \mathrm{H}^{5-8}\right) \mathrm{ppm}$;

${ }^{13} \mathrm{C}\left\{{ }^{1} \mathrm{H}\right\}$ NMR $\left(151 \mathrm{MHz}, \mathrm{CDCl}_{3}\right) \delta=73.7\left(1 \mathrm{C}, \mathrm{C}^{11}\right), 61.3\left(1 \mathrm{C}, \mathrm{C}^{2}\right), 58.4\left(1 \mathrm{C}, \mathrm{C}^{12}\right), 48.4$ (1C, $\left.C^{1}\right), 35.1\left(1 \mathrm{C}, \mathrm{C}^{3}\right), 29.4(1 \mathrm{C}), 29.4(1 \mathrm{C}), 29.0(2 \mathrm{C}), 28.8\left(1 \mathrm{C}, \mathrm{C}^{10}\right), 25.9\left(1 \mathrm{C}, \mathrm{C}^{4}\right)$, $25.5\left(1 \mathrm{C}, \mathrm{C}^{9}\right) \mathrm{ppm}$;

IR (ATR) $\tilde{v}=2928(\mathrm{~m}), 2856(\mathrm{w}), 1462(\mathrm{w}), 1396(\mathrm{~m}), 1385(\mathrm{~m}), 1296(\mathrm{w}), 1264(\mathrm{w})$, $1194(\mathrm{~s}), 998(\mathrm{~m}), 947(\mathrm{~s}), 826(\mathrm{~m}), 799(\mathrm{~m}), 772(\mathrm{~m}), 724(\mathrm{~m}), 661(\mathrm{~m}) \mathrm{cm}^{-1}$;

HRMS (ESI) $m / z$ [M+Na] ${ }^{+}$calcd. for $\mathrm{C}_{12} \mathrm{H}_{24} \mathrm{Cl}_{2} \mathrm{NaO}_{4} \mathrm{~S}^{+}: 357.0665$, found: 357.0668 .

\section{0,11-Dichloroundecyl 4-methylbenzenesulfonate (9)}

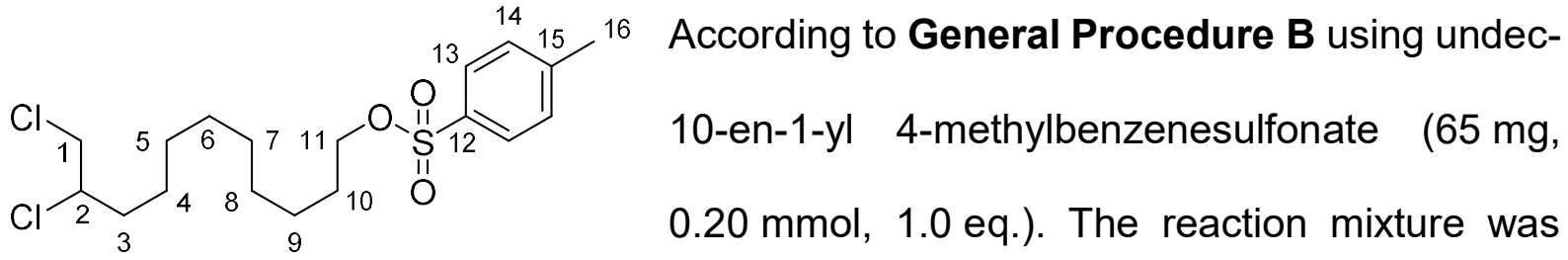

stirred at $0^{\circ} \mathrm{C}$ for $8 \mathrm{~h}$. The crude mixture was purified by column chromatography using $n$-pentane: $\mathrm{CH}_{2} \mathrm{Cl}_{2}$ 4:1 to afford 10,11-dichloroundecyl 4-methylbenzenesulfonate (9) as a colourless oil ( $57 \mathrm{mg}, 0.14 \mathrm{mmol}, 72 \%)$.

Rf: (n-pentane: $\mathrm{CH}_{2} \mathrm{Cl}_{2}$ 1:1) 0.36 ; 
${ }^{1} \mathrm{H}$ NMR $\left(500 \mathrm{MHz}, \mathrm{CDCl}_{3}\right) \delta=7.79\left(\mathrm{~m}, 2 \mathrm{H}, \mathrm{H}^{13}\right), 7.34\left(\mathrm{~m}, 2 \mathrm{H}, \mathrm{H}^{14}\right), 4.03\left(\mathrm{~m}, 1 \mathrm{H}, \mathrm{H}^{2}\right)$, $4.02\left(\mathrm{t},{ }^{3} \mathrm{JHH}_{\mathrm{HH}}=7.7 \mathrm{~Hz}, 2 \mathrm{H}, \mathrm{H}^{11}\right), 3.76\left(\mathrm{dd},{ }^{2} \mathrm{~J}_{\mathrm{HH}}=11.3 \mathrm{~Hz},{ }^{3} \mathrm{~J}_{\mathrm{HH}}=5.2 \mathrm{~Hz}, 1 \mathrm{H}, \mathrm{H}^{1 \mathrm{a}}\right), 3.65$ $\left(\mathrm{dd},{ }^{2} \mathrm{~J}_{\mathrm{HH}}=11.3 \mathrm{~Hz},{ }^{3} \mathrm{JHH}_{\mathrm{HH}}=7.4 \mathrm{~Hz}, 1 \mathrm{H}, \mathrm{H}^{1 \mathrm{~b}}\right), 2.45\left(\mathrm{~s}, 3 \mathrm{H}, \mathrm{H}^{16}\right), 1.97$ (dddd, ${ }^{2} \mathrm{JHH}_{\mathrm{HH}}=$ $\left.14.1 \mathrm{~Hz},{ }^{3} \mathrm{JHH}_{\mathrm{H}}=10.2,5.8,3.8 \mathrm{~Hz}, 1 \mathrm{H}, \mathrm{H}^{3 a}\right), 1.70\left(\mathrm{~m}, 1 \mathrm{H} ; \mathrm{H}^{3 b}\right), 1.63\left(\mathrm{~m}, 2 \mathrm{H}, \mathrm{H}^{10}\right), 1.55$ $\left(m, 1 H, H^{4 a}\right), 1.39\left(m, 1 H, H^{4 b}\right), 1.35-1.18\left(m, 10 H, H^{5-9}\right) p p m ;$

${ }^{13} \mathrm{C}\left\{{ }^{1} \mathrm{H}\right\}$ NMR $\left(126 \mathrm{MHz}, \mathrm{CDCl}_{3}\right) \delta=144.8\left(1 \mathrm{C}, \mathrm{C}^{15}\right), 133.4\left(1 \mathrm{C}, \mathrm{C}^{12}\right), 129.9\left(2 \mathrm{C}, \mathrm{C}^{14}\right)$, $128.0\left(2 \mathrm{C}, \mathrm{C}^{13}\right), 70.8\left(1 \mathrm{C}, \mathrm{C}^{11}\right), 61.4\left(1 \mathrm{C}, \mathrm{C}^{2}\right), 48.4\left(1 \mathrm{C}, \mathrm{C}^{1}\right), 35.2\left(1 \mathrm{C}, \mathrm{C}^{3}\right), 29.4(2 \mathrm{C})$, $29.0(2 \mathrm{C}), 25.5(1 \mathrm{C}), 28.9\left(1 \mathrm{C}, \mathrm{C}^{10}\right), 25.9\left(1 \mathrm{C}, \mathrm{C}^{4}\right), 21.8\left(1 \mathrm{C}, \mathrm{C}^{16}\right) \mathrm{ppm}$;

IR (ATR) $\tilde{v}=2927(\mathrm{~m}), 2857(\mathrm{w}), 1599(\mathrm{w}), 1464(\mathrm{w}), 1357(\mathrm{~m}), 1289(\mathrm{w}), 1262(\mathrm{w})$, $1219(w), 1188(m), 1174(s), 1098(m), 1020(m), 952(m), 813(m), 777(w), 724(w)$, $688(w), 662(s) \mathrm{cm}^{-1}$;

HRMS (ESI) $\mathrm{m} / z$ [M+Na] $]^{+}$calcd. for $\mathrm{C}_{18} \mathrm{H}_{28} \mathrm{Cl}_{2} \mathrm{NaO}_{3} \mathrm{~S}^{+}: 417.1028$, found: 417.1031 .

\section{0,11-Dichloroundecyl diphenyl phosphate (10)}

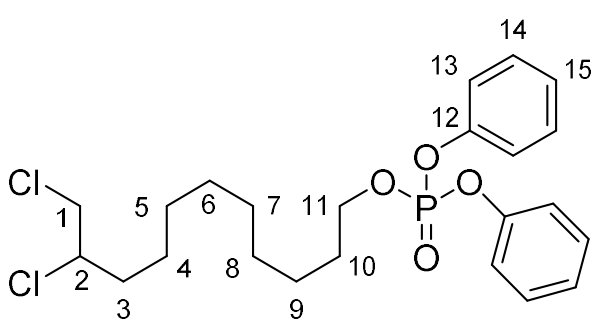

According to General Procedure B using diphenyl undec-10-en-1-yl phosphate $(80 \mathrm{mg}, 0.20 \mathrm{mmol}$, 1.0 eq.). The reaction mixture was stirred at $0^{\circ} \mathrm{C}$ for 8 h. The crude mixture was purified by column chromatography using a gradient $n$-pentane: $\mathrm{CH}_{2} \mathrm{Cl}_{2} 1: 1$ to $100 \% \mathrm{CH}_{2} \mathrm{Cl}_{2}$ to afford 10,11-dichloroundecyl diphenyl phosphate (10) as a pale yellow oil $(72 \mathrm{mg}, 0.15 \mathrm{mmol}$, $76 \%)$

Rf: (n-pentane: $\mathrm{CH}_{2} \mathrm{Cl}_{2}$ 1:1) 0.1;

${ }^{1} \mathrm{H}$ NMR $\left(500 \mathrm{MHz}, \mathrm{CDCl}_{3}\right) \delta=7.34\left(\mathrm{~m}, 4 \mathrm{H}, \mathrm{H}^{14}\right), 7.22\left(\mathrm{~m}, 4 \mathrm{H}, \mathrm{H}^{13}\right), 7.19\left(\mathrm{~m}, 2 \mathrm{H}, \mathrm{H}^{15}\right)$, $4.25\left(\mathrm{dt},{ }^{3} \mathrm{~J}_{\mathrm{PH}}=7.6 \mathrm{~Hz},{ }^{3} \mathrm{~J}_{\mathrm{HH}}=6.5 \mathrm{~Hz}, 2 \mathrm{H}, \mathrm{H}^{11}\right), 4.03\left(\mathrm{dddd},{ }^{3} \mathrm{~J}_{\mathrm{HH}}=9.0,7.4,5.2,3.8 \mathrm{~Hz}\right.$, $\left.1 \mathrm{H}, \mathrm{H}^{2}\right), 3.76\left(\mathrm{dd},{ }^{2} \mathrm{~J}_{\mathrm{HH}}=11.3 \mathrm{~Hz},{ }^{3} \mathrm{~J}_{\mathrm{HH}}=5.2 \mathrm{~Hz}, 1 \mathrm{H}, \mathrm{H}^{1 \mathrm{a}}\right), 3.65\left(\mathrm{dd},{ }^{2} \mathrm{~J}_{\mathrm{HH}}=11.3 \mathrm{~Hz}\right.$, 
$\left.{ }^{3} J_{H H}=7.4 \mathrm{~Hz}, 1 \mathrm{H}, \mathrm{H}^{1 \mathrm{~b}}\right), 1.98\left(\mathrm{dddd}, J=14.2,10.3,5.8 \mathrm{~Hz},{ }^{3} J_{\mathrm{HH}}=3.8 \mathrm{~Hz}, 1 \mathrm{H}, \mathrm{H}^{3 \mathrm{a}}\right.$ ), $1.70\left(\mathrm{~m}, 3 \mathrm{H}, \mathrm{H}^{3 \mathrm{~b}}, \mathrm{H}^{10}\right), 1.53\left(\mathrm{~m}, 1 \mathrm{H}, \mathrm{H}^{4 \mathrm{a}}\right), 1.41\left(\mathrm{~m}, 1 \mathrm{H}, \mathrm{H}^{4 \mathrm{~b}}\right), 1.37-1.22(\mathrm{~m}, 10 \mathrm{H}) \mathrm{ppm}$; ${ }^{13} \mathrm{C}\left\{{ }^{1} \mathrm{H}\right\}$ NMR $\left(126 \mathrm{MHz}, \mathrm{CDCl}_{3}\right) \delta=150.8\left(\mathrm{~d},{ }^{2} \mathrm{JPC}=7.2 \mathrm{~Hz}, 2 \mathrm{C}, \mathrm{C}^{12}\right), 129.9(\mathrm{~d}$, $\left.{ }^{4} J_{P C}=0.7 \mathrm{~Hz}, 4 \mathrm{C}, \mathrm{C}^{14}\right), 125.4\left(\mathrm{~d}, \mathrm{JPC}=1.2 \mathrm{~Hz}, 2 \mathrm{C}, \mathrm{C}^{15}\right), 120.2\left(\mathrm{~d},{ }^{3} \mathrm{JPC}_{\mathrm{PC}}=5.0 \mathrm{~Hz}, 4 \mathrm{C}\right.$, $\left.\mathrm{C}^{13}\right), 69.6\left(\mathrm{~d}, \mathrm{JPC}_{\mathrm{PC}}=6.6 \mathrm{~Hz}, 1 \mathrm{C}, \mathrm{C}^{11}\right), 61.4\left(1 \mathrm{C}, \mathrm{C}^{2}\right), 48.4\left(1 \mathrm{C}, \mathrm{C}^{1}\right), 35.2\left(1 \mathrm{C}, \mathrm{C}^{3}\right), 30.3$ $\left(\mathrm{d}, \mathrm{JPC}_{\mathrm{PC}}=6.8 \mathrm{~Hz}, 1 \mathrm{C}, \mathrm{C}^{10}\right), 29.4(2 \mathrm{C}), 29.1(2 \mathrm{C}), 25.9\left(1 \mathrm{C}, \mathrm{C}^{4}\right), 25.4\left(1 \mathrm{C}, \mathrm{C}^{9}\right) \mathrm{ppm}$;

${ }^{31} \mathrm{P}$ NMR $\left(202 \mathrm{MHz}, \mathrm{CDCl}_{3}\right) \delta=-11.85\left(\mathrm{t},{ }^{3} \mathrm{JPH}=7.6 \mathrm{~Hz}\right) \mathrm{ppm}$;

IR (ATR) $\tilde{v}=2927(\mathrm{w}), 2856(\mathrm{w}), 1591(\mathrm{w}), 1488(\mathrm{~m}), 1457(\mathrm{w}), 1287(\mathrm{~m}), 1220(\mathrm{~m})$, $1189(\mathrm{~s}), 1162(\mathrm{~m}), 1023(\mathrm{~m}), 1008(\mathrm{~m}), 941(\mathrm{~s}), 903(\mathrm{~m}), 824(\mathrm{w}), 754(\mathrm{~m}), 724(\mathrm{w})$, $688(\mathrm{~m}), 660(\mathrm{w}) \mathrm{cm}^{-1}$;

HRMS (ESI) $m / z$ [M+H] $]^{+}$calcd. for $\mathrm{C}_{23} \mathrm{H}_{32} \mathrm{O}_{4} \mathrm{PCl}_{2}{ }^{+}:$473.1410, found: 473.1411 .

\section{2-(4,5-Dichloropentyl)isoindoline-1,3-dione (11)}

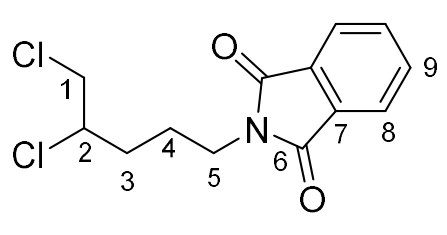

According to General Procedure B using 2-(pent-4-en-1yl)isoindoline-1,3-dione (43 mg, $0.20 \mathrm{mmol}, 1.0$ eq.). The reaction mixture was stirred at $0^{\circ} \mathrm{C}$ for $8 \mathrm{~h}$. The crude mixture was purified by column chromatography using $n$-pentane:ethyl acetate $9: 1$ to afford 2(4,5-dichloropentyl)isoindoline-1,3-dione (11) as a white solid $(34 \mathrm{mg}, 0.12 \mathrm{mmol}$, $59 \%)$.

Rf: (n-pentane: $\left.\mathrm{CH}_{2} \mathrm{Cl}_{2} 1: 1\right) 0.36$;

M.p.: $53-55^{\circ} \mathrm{C}$;

${ }^{1} \mathrm{H}$ NMR $\left(400 \mathrm{MHz}, \mathrm{CDCl}_{3}\right) \delta=7.85\left(\mathrm{~m}, 2 \mathrm{H}, \mathrm{H}^{8}\right), 7.72\left(\mathrm{~m}, 2 \mathrm{H}, \mathrm{H}^{9}\right), 4.09\left(\mathrm{dddd},{ }^{3} \mathrm{JHH}_{\mathrm{H}}=\right.$ 10.9, 8.3, 5.0, 3.0 Hz, 1H, $\left.\mathrm{H}^{2}\right), 3.74\left(\mathrm{~m}, 3 \mathrm{H}, \mathrm{H}^{1 \mathrm{a}}, \mathrm{H}^{5}\right), 3.64\left(\mathrm{~m}, 1 \mathrm{H}, \mathrm{H}^{1 \mathrm{~b}}\right), 2.02(\mathrm{~m}, 2 \mathrm{H}$, $\left.\mathrm{H}^{3 \mathrm{a}}, \mathrm{H}^{4 \mathrm{a}}\right), 1.79\left(\mathrm{~m}, 2 \mathrm{H}, \mathrm{H}^{3 \mathrm{~b}}, \mathrm{H}^{4 b}\right) \mathrm{ppm}$; 
${ }^{13} \mathrm{C}\left\{{ }^{1} \mathrm{H}\right\}$ NMR $\left(101 \mathrm{MHz}, \mathrm{CDCl}_{3}\right) \delta=168.5\left(2 \mathrm{C}, \mathrm{C}^{6}\right), 134.2\left(2 \mathrm{C}, \mathrm{C}^{9}\right), 132.2\left(2 \mathrm{C}, \mathrm{C}^{7}\right)$, $123.4\left(2 \mathrm{C}, \mathrm{C}^{8}\right), 60.4\left(1 \mathrm{C}, \mathrm{C}^{2}\right), 48.2\left(1 \mathrm{C}, \mathrm{C}^{1}\right), 37.2\left(1 \mathrm{C}, \mathrm{C}^{5}\right), 32.3\left(1 \mathrm{C}, \mathrm{C}^{3}\right), 25.3\left(1 \mathrm{C}, \mathrm{C}^{4}\right)$ ppm;

IR (ATR) $\tilde{v}=2949$ (w), $1771(\mathrm{~m}), 1703$ (s), 1615 (w), 1467 (w), $1436(\mathrm{w}), 1395(\mathrm{~s})$, $1362(m), 1288(w), 1219(w), 1189(m), 1122(w), 1102(w), 1088(w), 1021(m), 960$ $(w), 889(m), 879(m), 794(w), 716(s), 693(m), 662(m) \mathrm{cm}^{-1}$;

HRMS (ESI) $\mathrm{m} / z$ [M+Na] ${ }^{+}$calcd. for $\mathrm{C}_{13} \mathrm{H}_{13} \mathrm{NCl}_{2} \mathrm{NaO}_{2}{ }^{+}: 308.0216$, found: 308.0213 .

\section{1-(2,3-dichloropropoxy)-4-nitrobenzene (12)}

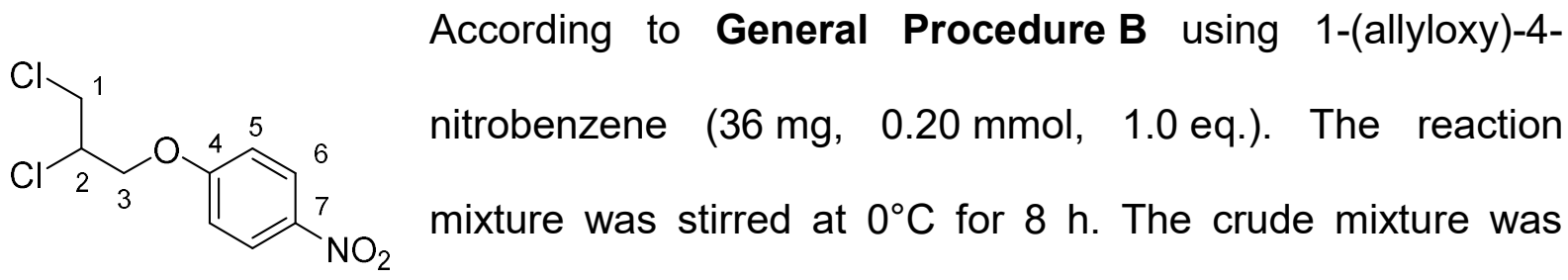
purified by column chromatography using cyclohexane:ethyl acetate 9:1 to afford 1(2,3-dichloropropoxy)-4-nitrobenzene (12) as a light yellow oil $(33 \mathrm{mg}, 0.13 \mathrm{mmol}$, $66 \%)$.

Rf: (cyclohexane:ethyl acetate 95:5) 0.23;

${ }^{1} \mathrm{H}$ NMR $\left(400 \mathrm{MHz}, \mathrm{CDCl}_{3}\right) \delta=8.23\left(\mathrm{~m}, 2 \mathrm{H}, \mathrm{H}^{6}\right), 7.01\left(\mathrm{~m}, 2 \mathrm{H}, \mathrm{H}^{5}\right), 4.39\left(\mathrm{~m}, 2 \mathrm{H}, \mathrm{H}^{3}\right)$, $4.32\left(\mathrm{~m}, 1 \mathrm{H}, \mathrm{H}^{2}\right), 3.94\left(\mathrm{~m}, 2 \mathrm{H}, \mathrm{H}^{1}\right) \mathrm{ppm}$;

${ }^{13} \mathrm{C}\left\{{ }^{1} \mathrm{H}\right\}$ NMR $\left(101 \mathrm{MHz}, \mathrm{CDCl}_{3}\right) \delta=162.9\left(1 \mathrm{C}, \mathrm{C}^{4}\right), 142.4\left(1 \mathrm{C}, \mathrm{C}^{7}\right), 126.2\left(2 \mathrm{C}, \mathrm{C}^{6}\right)$, $114.8\left(2 \mathrm{C}, \mathrm{C}^{5}\right), 68.7\left(1 \mathrm{C}, \mathrm{C}^{3}\right), 56.7\left(1 \mathrm{C}, \mathrm{C}^{2}\right), 44.5\left(1 \mathrm{C}, \mathrm{C}^{1}\right) \mathrm{ppm}$;

IR (ATR) $\tilde{v}=2956(\mathrm{w}), 1609(\mathrm{w}), 1593(\mathrm{~m}), 1514(\mathrm{~m}), 1497(\mathrm{~m}), 1455(\mathrm{w}), 1365(\mathrm{~m})$, $1341(\mathrm{~s}), 1299(\mathrm{~m}), 1256(\mathrm{~s}), 1190(\mathrm{~m}), 1175(\mathrm{~s}), 1112(\mathrm{~m}), 1097(\mathrm{~s}), 1020(\mathrm{~m}), 1002$ (m), $980(m), 932(m), 862(m), 845(m), 830(m), 814(m), 792(m), 752(m), 688(m)$, $667(\mathrm{~m}) \mathrm{cm}^{-1}$; 
HRMS (ESI) $m / z$ [M+Na] $]^{+}$calcd. for $\mathrm{C}_{9} \mathrm{H}_{9} \mathrm{Cl}_{2} \mathrm{NNaO}_{3}{ }^{+}: 271.9852$, found: 271.9856 .

\section{2,3-Dichloropropyl 4-methylbenzenesulfonate (13)}<smiles>Cc1ccc(S(=O)(=O)OCC(Cl)CCl)cc1</smiles>

According to General Procedure B using allyl 4methylbenzenesulfonate (43 $\mathrm{mg}, 0.20 \mathrm{mmol}, 1.0$ eq.). The reaction mixture was stirred at $0^{\circ} \mathrm{C}$ for $8 \mathrm{~h}$. The crude mixture was purified by column chromatography using $n$-pentane:ethyl acetate $95: 5$ to afford 2,3-dichloropropyl 4-methylbenzenesulfonate (13) as a colourless oil (30 mg, $0.11 \mathrm{mmol}, 53 \%)$.

Rf: (n-pentane:ethyl acetate 95:5) 0.17 ;

${ }^{1} \mathrm{H}$ NMR $\left(400 \mathrm{MHz}, \mathrm{CDCl}_{3}\right) \delta=7.81\left(\mathrm{~m}, 2 \mathrm{H}, \mathrm{H}^{5}\right), 7.37\left(\mathrm{~m}, 2 \mathrm{H}, \mathrm{H}^{6}\right), 4.32\left(\mathrm{dd},{ }^{2} \mathrm{JHH}_{\mathrm{H}}=\right.$ $\left.10.5 \mathrm{~Hz},{ }^{3} \mathrm{JHH}_{\mathrm{HH}}=5.2 \mathrm{~Hz}, 1 \mathrm{H}, \mathrm{H}^{3 \mathrm{a}}\right), 4.26\left(\mathrm{dd},{ }^{2} \mathrm{~J}_{\mathrm{HH}}=10.6 \mathrm{~Hz},{ }^{3} \mathrm{JHH}_{\mathrm{HH}}=5.1 \mathrm{~Hz}, 1 \mathrm{H}, \mathrm{H}^{3 \mathrm{~b}}\right), 4.21$ $\left(\mathrm{dq},{ }^{3} \mathrm{~J}_{\mathrm{HH}}=6.7,5.1 \mathrm{~Hz}, 1 \mathrm{H}, \mathrm{H}^{2}\right), 3.78\left(\mathrm{dd},{ }^{2}{ }_{\mathrm{HH}}=11.8 \mathrm{~Hz},{ }^{3} J_{\mathrm{HH}}=4.8 \mathrm{~Hz}, 1 \mathrm{H}, \mathrm{H}^{1 \mathrm{a}}\right), 3.74$ $\left(\mathrm{dd},{ }^{2} \mathrm{JHH}_{\mathrm{HH}}=11.8 \mathrm{~Hz},{ }^{3} \mathrm{JHH}_{\mathrm{HH}}=6.4 \mathrm{~Hz}, 1 \mathrm{H}, \mathrm{H}^{1 \mathrm{~b}}\right), 2.46\left(\mathrm{~s}, 3 \mathrm{H}, \mathrm{H}^{8}\right) \mathrm{ppm}$;

${ }^{13} \mathrm{C}\left\{{ }^{1} \mathrm{H}\right\}$ NMR $\left(101 \mathrm{MHz}, \mathrm{CDCl}_{3}\right) \delta=145.6\left(1 \mathrm{C}, \mathrm{C}^{7}\right), 132.3\left(1 \mathrm{C}, \mathrm{C}^{4}\right), 130.2\left(2 \mathrm{C}, \mathrm{C}^{6}\right)$, $128.2\left(2 \mathrm{C}, \mathrm{C}^{5}\right), 68.7\left(1 \mathrm{C}, \mathrm{C}^{3}\right), 55.9\left(1 \mathrm{C}, \mathrm{C}^{2}\right), 44.3\left(1 \mathrm{C}, \mathrm{C}^{1}\right), 21.8\left(1 \mathrm{C}, \mathrm{C}^{8}\right) \mathrm{ppm}$;

IR (ATR) $\tilde{v}=2958(w), 1598(w), 1495(w), 1453(w), 1362(m), 1308(w), 1293(w)$, $1212(w), 1190(m), 1174(s), 1096(m), 1039(w), 978(m), 933(m), 828(m), 812(m)$, $792(\mathrm{~m}), 745(\mathrm{w}), 681(\mathrm{~m}), 666(\mathrm{~m}) \mathrm{cm}^{-1}$;

HRMS (ESI) $\mathrm{m} / z$ [M+Na] $]^{+}$calcd. for $\mathrm{C}_{10} \mathrm{H}_{12} \mathrm{Cl}_{2} \mathrm{NaO}_{3} \mathrm{~S}^{+}: 304.9776$, found: 304.9783 . 


\section{1-chloro-4-(1,2-dichloroethyl)benzene (14)}

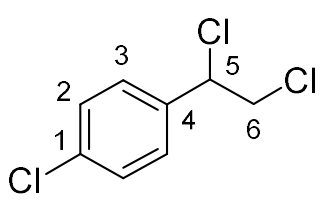

According to General Procedure B using 1-chloro-4-vinylbenzene (139 mg, $1.0 \mathrm{mmol}, 1.0$ eq.), p-iodotoluene (44 mg, $0.2 \mathrm{mmol}$, 0.2 eq.), cesium chloride (505 mg, $3.0 \mathrm{mmol}, 3.0$ eq.) and Selectfluor $^{\circledR}$ (390 mg, $1.1 \mathrm{mmol}, 1.1$ eq.) were dissolved in a solution of HFIP (1.51 g, $9.0 \mathrm{mmol}, 9.0$ eq. $)$ in $\mathrm{CH}_{2} \mathrm{Cl}_{2}(4.5 \mathrm{~mL})$. The reaction mixture was stirred at $0^{\circ} \mathrm{C}$ for $8 \mathrm{~h}$. The crude mixture was purified by column chromatography using $100 \%$ cyclohexane to afford 1-chloro-4-(1,2-dichloroethyl)benzene (14) as a colourless oil (112 mg, $0.53 \mathrm{mmol}, 53 \%)$.

Rf: (cyclohexane 100\%) 0.44;

${ }^{1} \mathrm{H}$ NMR $\left(300 \mathrm{MHz}, \mathrm{CDCl}_{3}\right) \delta=7.36(\mathrm{~m}, 4 \mathrm{H}), 4.97\left(\mathrm{dd},{ }^{3} \mathrm{~J}_{\mathrm{HH}}=8.4,6.2 \mathrm{~Hz}, 1 \mathrm{H}\right), 3.99$ $\left(\mathrm{dd},{ }^{2} \mathrm{~J}_{\mathrm{HH}}=11.3 \mathrm{~Hz},{ }^{3} \mathrm{~J}_{\mathrm{HH}}=6.2 \mathrm{~Hz}, 1 \mathrm{H}\right), 3.88\left(\mathrm{dd},{ }^{2} \mathrm{~J}_{\mathrm{HH}}=11.3 \mathrm{~Hz},{ }^{3} \mathrm{~J}_{\mathrm{HH}}=8.4 \mathrm{~Hz}, 1 \mathrm{H}\right)$.

MS (EI) m/z: $207.98(40)[\mathrm{M}]^{+}, 173.03(100)[\mathrm{M}-\mathrm{Cl}]^{+}$.

The analytic data are in agreement with the literature. ${ }^{15}$

\section{1-bromo-4-(1,2-dichloroethyl)benzene (15)}<smiles>ClCC(Cl)c1ccc(Br)cc1</smiles>

According to General Procedure B using 1-bromo-4-vinylbenzene (183 mg, $1.0 \mathrm{mmol}, 1.0$ eq.), p-iodotoluene (44 mg, $0.2 \mathrm{mmol}$, 0.2 eq.), cesium chloride (505 $\mathrm{mg}, 3.0 \mathrm{mmol}, 3.0$ eq.) and Selectfluor ${ }^{\circledR}$ (390 mg, $1.1 \mathrm{mmol}, 1.1$ eq.) were dissolved in a solution of HFIP $(1.51 \mathrm{~g}$, $9.0 \mathrm{mmol}, 9.0$ eq. $)$ in $\mathrm{CH}_{2} \mathrm{Cl}_{2}(4.5 \mathrm{~mL})$. The reaction mixture was stirred at $0^{\circ} \mathrm{C}$ for $8 \mathrm{~h}$. The crude mixture was purified by column chromatography using $100 \%$ cyclohexane to afford 1-bromo-4-(1,2-dichloroethyl)benzene (15) as a colourless oil (146 mg, $0.57 \mathrm{mmol}, 57 \%)$.

Rf: (cyclohexane 100\%) 0.45; 
${ }^{1} \mathrm{H}$ NMR $\left(300 \mathrm{MHz}, \mathrm{CDCl}_{3}\right) \delta=7.53(\mathrm{~m}, 2 \mathrm{H}), 7.29(\mathrm{~m}, 2 \mathrm{H}), 4.95\left(\mathrm{dd},{ }^{3} \mathrm{JHH}_{\mathrm{H}}=8.4,6.1 \mathrm{~Hz}\right.$, $1 \mathrm{H}), 3.98\left(\mathrm{dd},{ }^{2} \mathrm{~J}_{\mathrm{HH}}==11.3 \mathrm{~Hz},{ }^{3} J_{\mathrm{HH}}=6.2 \mathrm{~Hz}, 1 \mathrm{H}\right), 3.88\left(\mathrm{dd},{ }^{2} J_{\mathrm{HH}}=11.3 \mathrm{~Hz},{ }^{3} J_{\mathrm{HH}}=\right.$ 8.4 Hz, 1H).

MS (EI) m/z: 251.94 (12). 253.93 (22), 255.93 (8) [M] ${ }^{+}, 202.94$ (77), 204.94 (100), $206.95(24)\left[\mathrm{M}-\mathrm{CH}_{2} \mathrm{Cl}\right]^{+}$.

The analytic data are in agreement with the literature. ${ }^{17}$

\section{2-(1,2-dichloroethyl)-1,1'-biphenyl (16)}

According to General Procedure B using 2-vinyl-1,1'-biphenyl
$(180 \mathrm{mg}, 1.0 \mathrm{mmol}, 1.0$ eq. $), p$-iodotoluene (44 mg, $0.2 \mathrm{mmol}$, Selectfluor $^{\circledR}$ (390 mg, $1.1 \mathrm{mmol}, 1.1$ eq.) were dissolved in a solution of HFIP (1.51 g, $9.0 \mathrm{mmol}, 9.0$ eq.) in $\mathrm{CH}_{2} \mathrm{Cl}_{2}(4.5 \mathrm{~mL})$. The reaction mixture was stirred at $0^{\circ} \mathrm{C}$ for $8 \mathrm{~h}$. The crude mixture was purified by column chromatography using $100 \%$ cyclohexane to afford 2-(1,2-dichloroethyl)-1,1'-biphenyl (16) as a colourless oil (147 mg, $0.59 \mathrm{mmol}, 59 \%)$.

Rf: (cyclohexane 100\%) 0.28;

${ }^{1} \mathrm{H}$ NMR $\left(500 \mathrm{MHz}, \mathrm{CDCl}_{3}\right) \delta=7.61(\mathrm{dd}, J=7.9,1.1 \mathrm{~Hz}, 1 \mathrm{H}), 7.49-7.35(\mathrm{~m}, 7 \mathrm{H}), 7.28$ (dd, $J=7.6,1.4 \mathrm{~Hz}, 1 \mathrm{H}), 5.16\left(\mathrm{t},{ }^{3} \mathrm{~J}_{\mathrm{HH}}=7.5 \mathrm{~Hz}, 1 \mathrm{H}\right), 3.97-3.92(\mathrm{~m}, 2 \mathrm{H})$.

MS (EI) m/z: 250.01 (100). 252.07 (65), 254.04 (10) [M] ${ }^{+}$

The analytic data are in agreement with the literature. ${ }^{18}$ 


\section{General procedure $\mathrm{C}$ for the catalytic dichlorination of internal alkenes}

The corresponding alkene $(0.20 \mathrm{mmol}, 1.0$ eq. $), p$-iodotoluene $(8.7 \mathrm{mg}, 0.04 \mathrm{mmol}$, 0.2 eq.) cesium chloride (101 mg, $0.60 \mathrm{mmol}, 3.0$ eq.) and Selectfluor ${ }^{\circledR}(78.0 \mathrm{mg}, 0.22$ mmol, 1.1 eq.) were dissolved in a solution of HFIP (302 mg, $1.80 \mathrm{mmol}, 9.0$ eq.) in $\mathrm{CH}_{2} \mathrm{Cl}_{2}(0.9 \mathrm{~mL})$ and stirred at ambient temperature. After $8 \mathrm{~h}$, the reaction mixture was quenched with a saturated solution of $\mathrm{Na}_{2} \mathrm{~S}_{2} \mathrm{O}_{3}$ and extracted with $\mathrm{CH}_{2} \mathrm{Cl}_{2}(3 x)$. The combined organic phases were dried over $\mathrm{MgSO}_{4}$, filtered and the solvent was evaporated under vacuo. The residue was dissolved in $0.6 \mathrm{~mL}$ of $\mathrm{CDCl}_{3}$ containing $0.1 \mathrm{mmol}$ of DMF (stock solution) and analysed by ${ }^{1} \mathrm{H}$ NMR. The sample was then evaporated under vacuo and purified by column chromatography using the indicated solvent ratio.

\section{4,5-Dichlorohexyl benzoate (17)}

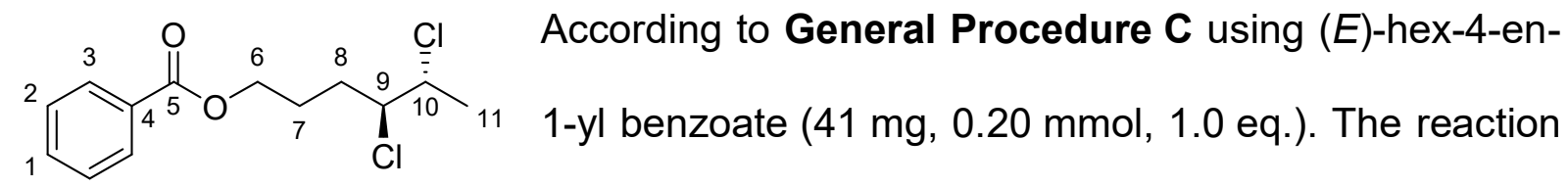
mixture was stirred at ambient temperature for $8 \mathrm{~h}$. The crude mixture was purified by column chromatography using $n$-pentane:diethyl ether 40:1 to afford 4,5-dichlorohexyl benzoate (17) as a colourless oil (30 mg, $0.11 \mathrm{mmol}, 54 \%)$.

Rf: (n-pentane:diethyl ether 10:1) 0.39;

${ }^{1} \mathrm{H}$ NMR $\left(400 \mathrm{MHz} \mathrm{CDCl}_{3}\right) \delta=8.04\left(\mathrm{~m}, 2 \mathrm{H}, \mathrm{H}^{3}\right), 7.56\left(\mathrm{~m}, 1 \mathrm{H}, \mathrm{H}^{1}\right), 7.45\left(\mathrm{~m}, 2 \mathrm{H}, \mathrm{H}^{2}\right)$, $4.37\left(\mathrm{~m}, 2 \mathrm{H}, \mathrm{H}^{6}\right), 4.11\left(\mathrm{qd},{ }^{3} \mathrm{~J}_{\mathrm{HH}}=6.9,6.6 \mathrm{~Hz}, 1 \mathrm{H}, \mathrm{H}^{10}\right), 4.00\left(\mathrm{ddd},{ }^{3} \mathrm{~J}_{\mathrm{HH}}=9.6,6.9\right.$, $\left.2.9 \mathrm{~Hz}, 1 \mathrm{H}, \mathrm{H}^{9}\right), 2.26-2.18\left(\mathrm{~m}, 1 \mathrm{H}, \mathrm{H}^{8 \mathrm{a}}\right), 2.15-2.07\left(\mathrm{~m}, 1 \mathrm{H}, \mathrm{H}^{7 \mathrm{a}}\right), 1.97-1.87(\mathrm{~m}, 2 \mathrm{H}$, $\left.\mathrm{H}^{7 b}, \mathrm{H}^{8 b}\right), 1.65\left(\mathrm{~d},{ }^{3} \mathrm{~J}_{\mathrm{HH}}=6.5 \mathrm{~Hz}, 3 \mathrm{H}, \mathrm{H}^{11}\right) \mathrm{ppm}$; 
${ }^{13} \mathrm{C}\left\{{ }^{1} \mathrm{H}\right\}$ NMR $\left(151 \mathrm{MHz}, \mathrm{CDCl}_{3}\right) \delta=166.7\left(1 \mathrm{C}, \mathrm{C}^{5}\right), 133.1\left(1 \mathrm{C}, \mathrm{C}^{1}\right), 130.3\left(1 \mathrm{C}, \mathrm{C}^{4}\right)$, $129.7\left(2 \mathrm{C}, \mathrm{C}^{3}\right), 128.5\left(2 \mathrm{C}, \mathrm{C}^{2}\right), 66.7\left(1 \mathrm{C}, \mathrm{C}^{9}\right), 64.2\left(1 \mathrm{C}, \mathrm{C}^{6}\right), 60.1\left(1 \mathrm{C}, \mathrm{C}^{10}\right), 31.9(1 \mathrm{C}$, $\left.\mathrm{C}^{8}\right), 25.7\left(1 \mathrm{C}, \mathrm{C}^{7}\right), 22.3\left(1 \mathrm{C}, \mathrm{C}^{11}\right) \mathrm{ppm}$;

IR (ATR) $\tilde{v}=3069(w), 2937(w), 2860(w), 1716(s), 1640(w), 1602(w), 1585(w)$, $1451(\mathrm{~m}), 1385(\mathrm{w}), 1268(\mathrm{~s}), 1175(\mathrm{~m}), 1109(\mathrm{~s}), 1069(\mathrm{~m}), 1026(\mathrm{~m}), 993(\mathrm{~m})$, $910(\mathrm{~m}), 847(\mathrm{w}), 707(\mathrm{~s}), 686(\mathrm{~m}) \mathrm{cm}^{-1}$;

HRMS (ESI) $m / z$ [M+Na] $]^{+}$calcd. for $\mathrm{C}_{13} \mathrm{H}_{16} \mathrm{O}_{2} \mathrm{Cl}_{2} \mathrm{Na}^{+}: 297.0420$, found: 297.0426 .

\section{4,5-Dichlorohexyl 4-methoxybenzoate (18)}

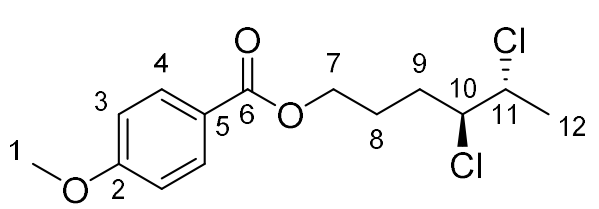

According to General Procedure C using (E)-hex-

4-en-1-yl 4-methoxybenzoate (47 mg, $0.20 \mathrm{mmol}$, 1.0 eq.). The reaction mixture was stirred at ambient temperature for $8 \mathrm{~h}$. The crude mixture was purified by column chromatography using n-pentane:diethyl ether 40:1 to afford 4,5-dichlorohexyl 4-methoxybenzoate (18) as a colourless oil ( $35 \mathrm{mg}, 0.11 \mathrm{mmol}, 57 \%)$.

Rf: (n-pentane:diethyl ether 10:1) 0.19;

${ }^{1} \mathrm{H}$ NMR $\left(400 \mathrm{MHz}, \mathrm{CDCl}_{3}\right) \delta=7.99\left(\mathrm{~m}, 2 \mathrm{H}, \mathrm{H}^{4}\right), 6.92\left(\mathrm{~m}, 2 \mathrm{H}, \mathrm{H}^{3}\right), 4.34\left(\mathrm{~m}, 2 \mathrm{H}, \mathrm{H}^{7}\right)$, $4.11\left(\mathrm{dq},{ }^{3} \mathrm{~J}_{\mathrm{HH}}=6.9,6.6 \mathrm{~Hz}, 1 \mathrm{H}, \mathrm{H}^{11}\right), 4.00\left(\mathrm{ddd},{ }^{3} \mathrm{H}_{\mathrm{HH}}=9.5,6.9,2.9 \mathrm{~Hz}, 1 \mathrm{H}, \mathrm{H}^{10}\right)$, $3.86\left(\mathrm{~s}, 3 \mathrm{H}, \mathrm{H}^{1}\right), 2.26-2.03\left(\mathrm{~m}, 2 \mathrm{H}, \mathrm{H}^{8 \mathrm{a}}, \mathrm{H}^{9 \mathrm{a}}\right), 1.95-1.83\left(\mathrm{~m}, 2 \mathrm{H}, \mathrm{H}^{8 \mathrm{~b}}, \mathrm{H}^{9 b}\right), 1.64(\mathrm{~d}$, $\left.J_{\mathrm{HH}}=6.6 \mathrm{~Hz}, 3 \mathrm{H}, \mathrm{H}^{12}\right) \mathrm{ppm}$;

${ }^{13} \mathrm{C}\left\{{ }^{1} \mathrm{H}\right\}$ NMR $\left(101 \mathrm{MHz}, \mathrm{CDCl}_{3}\right) \delta=166.4\left(1 \mathrm{C}, \mathrm{C}^{6}\right), 163.5\left(1 \mathrm{C}, \mathrm{C}^{2}\right), 131.7\left(2 \mathrm{C}, \mathrm{C}^{4}\right)$, $122.7\left(1 \mathrm{C}, \mathrm{C}^{5}\right), 113.8\left(2 \mathrm{C}, \mathrm{C}^{3}\right), 66.8\left(1 \mathrm{C}, \mathrm{C}^{10}\right), 63.9\left(1 \mathrm{C}, \mathrm{C}^{7}\right), 60.2\left(1 \mathrm{C}, \mathrm{C}^{11}\right), 55.6(1 \mathrm{C}$, $\left.\mathrm{C}^{1}\right), 31.9\left(1 \mathrm{C}, \mathrm{C}^{9}\right), 25.7\left(1 \mathrm{C}, \mathrm{C}^{8}\right), 22.3\left(1 \mathrm{C}, \mathrm{C}^{12}\right) \mathrm{ppm}$; 
IR (ATR) $\tilde{v}=3673(\mathrm{w}), 2966(\mathrm{w}), 1708(\mathrm{~s}), 1605(\mathrm{~s}), 1510(\mathrm{~m}), 1444(\mathrm{w}), 1420(\mathrm{w})$, $1381(w), 1273(s), 1251(s), 1165(s), 1110(s), 1027(s), 968(w), 920(w), 846(m)$, $768(\mathrm{~s}), 695(\mathrm{~m}) \mathrm{cm}^{-1}$;

HRMS (ESI) $m / z$ [M+Na] $]^{+}$calcd. for $\mathrm{C}_{14} \mathrm{H}_{18} \mathrm{O}_{3} \mathrm{Cl}_{2} \mathrm{Na}^{+}: 327.0525$, found: 327.0530 .

\section{4,5-Dichlorohexyl 3,5-dinitrobenzoate (19)}

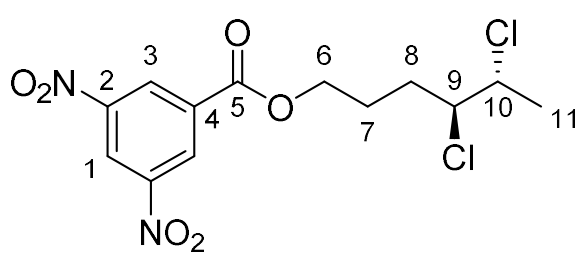

According to General Procedure C using (E)-hex-4en-1-yl 3,5-dinitrobenzoate $(59 \mathrm{mg}, \quad 0.20 \mathrm{mmol}$, 1.0 eq.). The reaction mixture was stirred at ambient temperature for $8 \mathrm{~h}$. The crude mixture was purified by column chromatography using n-pentane:diethyl ether 10:1 to afford 4,5-dichlorohexyl 3,5-dinitrobenzoate (19) as a yellow solid (41 mg, $0.11 \mathrm{mmol}, 56 \%)$.

Rf: ( $n$-pentane:diethyl ether 10:1) 0.13 ;

M.p. $51-53^{\circ} \mathrm{C}$;

${ }^{1} \mathrm{H} \mathrm{NMR}\left(400 \mathrm{MHz}, \mathrm{CDCl}_{3}\right) \delta=9.23\left(\mathrm{t},{ }^{4} \mathrm{JHH}=2.1 \mathrm{~Hz}, 1 \mathrm{H}, \mathrm{H}^{1}\right), 9.16\left(\mathrm{~d},{ }^{4} \mathrm{JHH}=2.1 \mathrm{~Hz}\right.$, $\left.2 \mathrm{H}, \mathrm{H}^{3}\right), 4.51\left(\mathrm{t},{ }^{3} \mathrm{JHH}_{\mathrm{H}}=6.2 \mathrm{~Hz}, 2 \mathrm{H}, \mathrm{H}^{6}\right), 4.11\left(\mathrm{dq},{ }^{3} \mathrm{JHH}_{\mathrm{HH}}=7.5,6.5 \mathrm{~Hz}, 1 \mathrm{H}, \mathrm{H}^{10}\right)$, $3.98\left(\mathrm{ddd},{ }^{3} \mathrm{JHH}_{\mathrm{HH}}=8.9,7.5,2.5 \mathrm{~Hz}, 1 \mathrm{H}, \mathrm{H}^{9}\right), 2.33-2.10\left(\mathrm{~m}, 2 \mathrm{H}, \mathrm{H}^{7 \mathrm{a}}, \mathrm{H}^{8 \mathrm{a}}\right), 2.08-$ $1.87\left(\mathrm{~m}, 2 \mathrm{H}, \mathrm{H}^{7 \mathrm{~b}}, \mathrm{H}^{8 b}\right), 1.67\left(\mathrm{~d},{ }^{3} \mathrm{JHH}=6.5 \mathrm{~Hz}, 3 \mathrm{H}, \mathrm{H}^{11}\right) \mathrm{ppm}$;

${ }^{13} \mathrm{C}\left\{{ }^{1} \mathrm{H}\right\}$ NMR $\left(101 \mathrm{MHz}, \mathrm{CDCl}_{3}\right) \delta=162.6\left(1 \mathrm{C}, \mathrm{C}^{5}\right), 148.9\left(2 \mathrm{C}, \mathrm{C}^{2}\right), 134.0\left(1 \mathrm{C}, \mathrm{C}^{4}\right)$, $129.6\left(2 \mathrm{C}, \mathrm{C}^{3}\right), 122.6\left(1 \mathrm{C}, \mathrm{C}^{1}\right), 66.3\left(1 \mathrm{C}, \mathrm{C}^{9}\right), 66.2\left(1 \mathrm{C}, \mathrm{C}^{6}\right), 60.0\left(1 \mathrm{C}, \mathrm{C}^{10}\right), 31.7(1 \mathrm{C}$, $\left.\mathrm{C}^{8}\right), 25.4\left(1 \mathrm{C}, \mathrm{C}^{7}\right), 22.7\left(1 \mathrm{C}, \mathrm{C}^{11}\right) \mathrm{ppm}$;

IR (ATR) $\tilde{v}=3095(\mathrm{w}), 1726(\mathrm{~s}), 1628(\mathrm{~m}), 1597(\mathrm{w}), 1542(\mathrm{~s}), 1462(\mathrm{~m}), 1343(\mathrm{~s})$, $1278(s), 1197(w), 1169(m), 1159(m), 1076(w), 985(w), 883(m), 827(w), 730(m)$, $718(\mathrm{~s}) \mathrm{cm}^{-1}$; 
HRMS (ESI) $m / z$ [M+Na] ${ }^{+}$calcd. for $\mathrm{C}_{13} \mathrm{H}_{14} \mathrm{~N}_{2} \mathrm{O}_{2} \mathrm{Cl}_{2} \mathrm{Na}^{+}: 387.0121$, found: 387.0118 .

\section{4,5-Dichlorohexyl benzoate (20)}

( According to General Procedure $C$ using (Z)-hex-4-enmixture was stirred at ambient temperature for $8 \mathrm{~h}$. The crude mixture was purified by column chromatography using $n$-pentane:diethyl ether $40: 1$ to afford 4,5-dichlorohexyl benzoate (20) as a colourless oil ( $25 \mathrm{mg}, 0.09 \mathrm{mmol}, 45 \%)$.

Rf: (n-pentane:diethyl ether 10:1) 0.29;

${ }^{1} \mathrm{H} \mathrm{NMR}\left(400 \mathrm{MHz}, \mathrm{CDCl}_{3}\right) \delta=8.04\left(\mathrm{~m}, 2 \mathrm{H}, \mathrm{H}^{3}\right), 7.56\left(\mathrm{~m}, 1 \mathrm{H}, \mathrm{H}^{1}\right), 7.45\left(\mathrm{~m}, 2 \mathrm{H}, \mathrm{H}^{2}\right)$, $4.38\left(\mathrm{~m}, 2 \mathrm{H}, \mathrm{H}^{6}\right), 4.28\left(\mathrm{qd},{ }^{3} \mathrm{~J}_{\mathrm{HH}}=6.7,3.0 \mathrm{~Hz}, 1 \mathrm{H}, \mathrm{H}^{10}\right), 4.09\left(\mathrm{td},{ }^{3} \mathrm{~J}_{\mathrm{HH}}=10.4,3.0 \mathrm{~Hz}\right.$, $\left.1 \mathrm{H}, \mathrm{H}^{9}\right), 2.18-2.04\left(\mathrm{~m}, 2 \mathrm{H}, \mathrm{H}^{7 \mathrm{a}}, \mathrm{H}^{8 \mathrm{a}}\right), 1.97-1.83\left(\mathrm{~m}, 2 \mathrm{H}, \mathrm{H}^{7 \mathrm{~b}}, \mathrm{H}^{8 \mathrm{~b}}\right), 1.60(\mathrm{~d}$, $\left.{ }^{3} \mathrm{~J}_{\mathrm{HH}}=6.7 \mathrm{~Hz}, 3 \mathrm{H}, \mathrm{H}^{11}\right) \mathrm{ppm}$;

${ }^{13} \mathrm{C}\left\{{ }^{1} \mathrm{H}\right\}$ NMR $\left(101 \mathrm{MHz}, \mathrm{CDCl}_{3}\right) \delta=166.7\left(1 \mathrm{C}, \mathrm{C}^{5}\right), 133.1\left(1 \mathrm{C}, \mathrm{C}^{1}\right), 130.3\left(1 \mathrm{C}, \mathrm{C}^{4}\right)$, $129.7\left(2 \mathrm{C}, \mathrm{C}^{3}\right), 128.5\left(2 \mathrm{C}, \mathrm{C}^{2}\right), 65.7\left(1 \mathrm{C}, \mathrm{C}^{9}\right), 64.2\left(1 \mathrm{C}, \mathrm{C}^{6}\right), 60.0\left(1 \mathrm{C}, \mathrm{C}^{10}\right), 30.6(1 \mathrm{C}$, $\left.\mathrm{C}^{8}\right), 26.2\left(1 \mathrm{C}, \mathrm{C}^{7}\right), 20.8\left(1 \mathrm{C}, \mathrm{C}^{11}\right) \mathrm{ppm}$;

IR (ATR) $\tilde{v}=2956(\mathrm{~m}), 1715(\mathrm{~s}), 1602(\mathrm{w}), 1584(\mathrm{w}), 1451(\mathrm{~m}), 1380(\mathrm{w}), 1314(\mathrm{~m})$, $1269(\mathrm{~s}), 1176(\mathrm{~m}), 1108(\mathrm{~s}), 1069(\mathrm{~m}), 1026(\mathrm{~m}), 981(\mathrm{w}), 915(\mathrm{w}), 806(\mathrm{w}), 708(\mathrm{~s})$, $687(\mathrm{~m}) \mathrm{cm}^{-1}$

HRMS (ESI) $m / z$ [M+Na] $]^{+}$calcd. for $\mathrm{C}_{13} \mathrm{H}_{16} \mathrm{O}_{2} \mathrm{Cl}_{2} \mathrm{Na}^{+}: 297.0420$, found: 297.0421 . 


\section{4,5-Dichlorohexyl 3,5-dinitrobenzoate (21)}

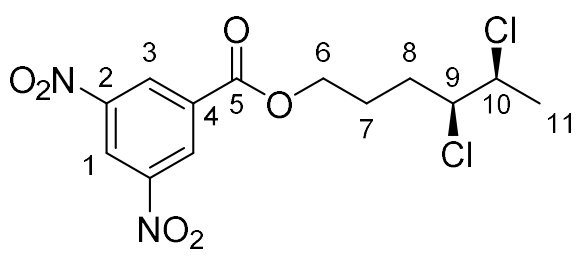

According to General Procedure C using (Z)-hex-

4-en-1-yl 3,5-dinitrobenzoate $(59 \mathrm{mg}, \quad 0.20 \mathrm{mmol}$, 1.0 eq.). The reaction mixture was stirred at ambient temperature for $8 \mathrm{~h}$. The crude mixture was purified by column chromatography using n-pentane:diethyl ether 10:1 to afford 4,5-dichlorohexyl 3,5-dinitrobenzoate (21) as a yellow solid (41 mg, $0.11 \mathrm{mmol}, 57 \%)$.

Rf: (n-pentane:diethyl ether 10:1) 0.06;

${ }^{1} \mathrm{H}$ NMR $\left(400 \mathrm{MHz}, \mathrm{CDCl}_{3}\right) \delta=9.24\left(\mathrm{t},{ }^{4} \mathrm{~J}_{\mathrm{HH}}=2.2 \mathrm{~Hz}, 1 \mathrm{H}, \mathrm{H}^{1}\right), 9.16\left(\mathrm{~d},{ }^{4} \mathrm{~J}_{\mathrm{HH}}=2.2 \mathrm{~Hz}\right.$, $\left.2 \mathrm{H}, \mathrm{H}^{3}\right), 4.51\left(\mathrm{~m}, 2 \mathrm{H}, \mathrm{H}^{6}\right), 4.30\left(\mathrm{qd},{ }^{3} \mathrm{~J}_{\mathrm{HH}}=6.7,3.0 \mathrm{~Hz}, 1 \mathrm{H}, \mathrm{H}^{10}\right), 4.10\left(\mathrm{dt},{ }^{3} \mathrm{~J}_{\mathrm{HH}}=10.1\right.$, $\left.3.0 \mathrm{~Hz}, 1 \mathrm{H}, \mathrm{H}^{9}\right), 2.26-2.06\left(\mathrm{~m}, 2 \mathrm{H}, \mathrm{H}^{7 \mathrm{a}}, \mathrm{H}^{8 \mathrm{a}}\right), 2.04-1.82\left(\mathrm{~m}, 2 \mathrm{H}, \mathrm{H}^{7 \mathrm{~b}}, \mathrm{H}^{8 \mathrm{~b}}\right), 1.61(\mathrm{~d}$, $\left.{ }^{3} J_{\mathrm{HH}}=6.7 \mathrm{~Hz}, 3 \mathrm{H}, \mathrm{H}^{11}\right) \mathrm{ppm}$;

${ }^{13} \mathrm{C}\left\{{ }^{1} \mathrm{H}\right\}$ NMR $\left(101 \mathrm{MHz}, \mathrm{CDCl}_{3}\right) \delta=162.6\left(1 \mathrm{C}, \mathrm{C}^{5}\right), 148.9\left(2 \mathrm{C}, \mathrm{C}^{2}\right), 134.0\left(1 \mathrm{C}, \mathrm{C}^{4}\right)$ $129.6\left(2 \mathrm{C}, \mathrm{C}^{3}\right), 122.6\left(1 \mathrm{C}, \mathrm{C}^{1}\right), 66.2\left(1 \mathrm{C}, \mathrm{C}^{6}\right), 65.3\left(1 \mathrm{C}, \mathrm{C}^{9}\right), 59.8\left(1 \mathrm{C}, \mathrm{C}^{10}\right), 30.1(1 \mathrm{C}$, $\left.C^{8}\right), 26.1\left(1 C, C^{7}\right), 20.5\left(1 C, C^{11}\right) p p m ;$

IR (ATR) $\tilde{v}=3102(\mathrm{w}), 2928(\mathrm{w}), 1731$ (s), $1629(\mathrm{~m}), 1540$ (s), $1342(\mathrm{~s}), 1273$ (s), 1163 (s), $1074(\mathrm{~m}), 1011(\mathrm{w}), 989(\mathrm{w}), 919(\mathrm{~m}), 772(\mathrm{~m}), 718(\mathrm{~s}), 694(\mathrm{~m}) \mathrm{cm}^{-1}$;

HRMS (ESI) $m / z$ [M+Na] ${ }^{+}$calcd. for $\mathrm{C}_{13} \mathrm{H}_{14} \mathrm{~N}_{2} \mathrm{O}_{2} \mathrm{Cl}_{2} \mathrm{Na}^{+}: 387.0121$, found: 387.0120 . 


\section{Mechanistic studies on the dichlorination of terminal alkenes}

a. Table S3: Exploring the role of HFIP in the dichlorination.

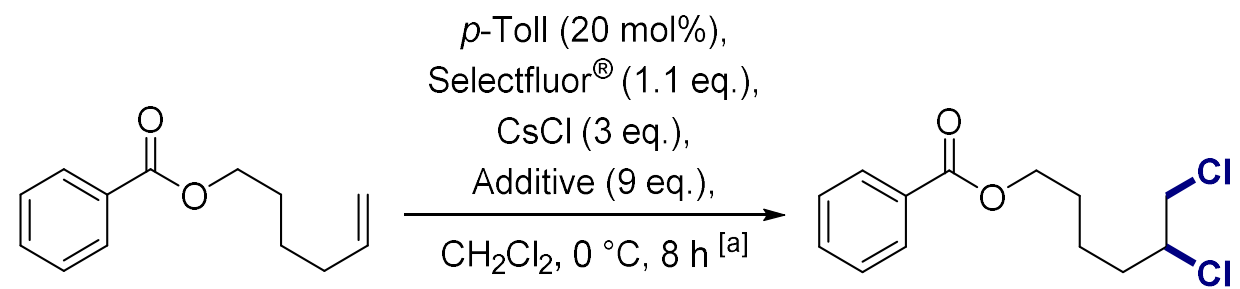

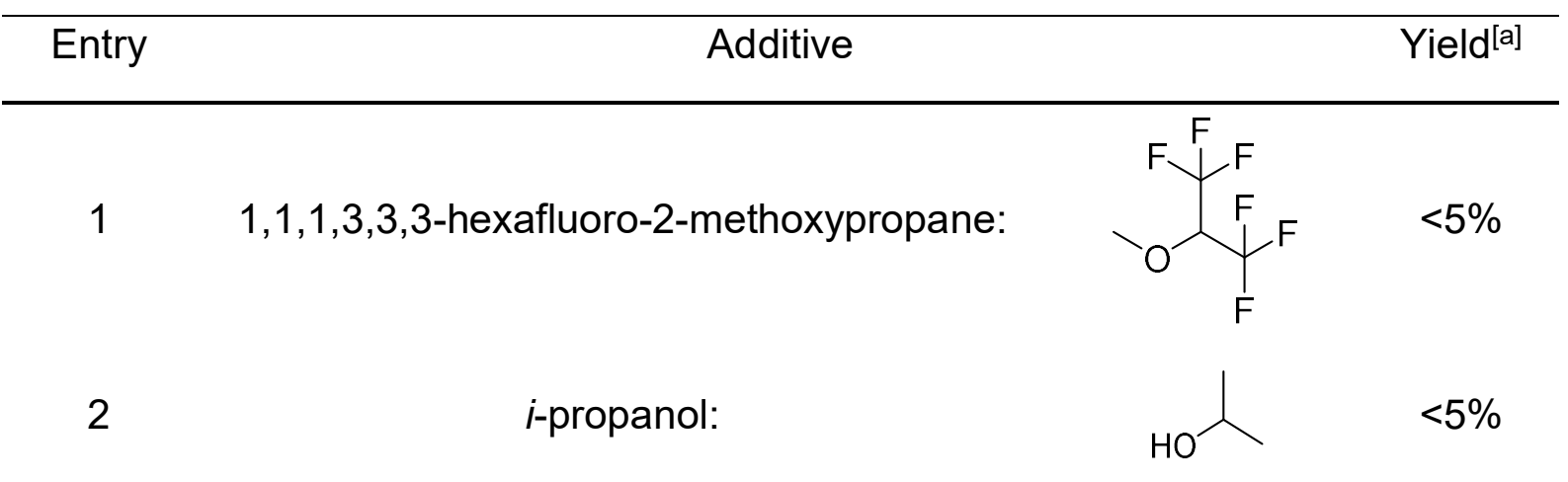

[a] According to General Procedure B using the corresponding additive instead of HFIP. ${ }^{1} \mathrm{H}$ NMR yield using DMF as internal standard.

b. Table S4: Radical clock experiments.<smiles>C=CCNCC(Cl)CCl</smiles>

S14

22

23

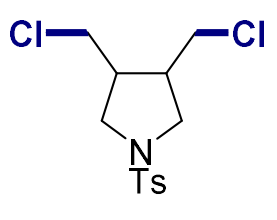

24

\begin{tabular}{|c|c|c|c|}
\hline Conditions & Yield 22 & Yield 23 & Yield 24 \\
\hline $\begin{array}{c}p \text {-Toll (20 mol\%), Selectfluor } \\
\text { (1.1 eq.), } \mathrm{CsCl} \text { (3.0 eq.), HFIP } \\
\text { (9.0 eq.), } \mathrm{CH}_{2} \mathrm{Cl}_{2}, 0^{\circ} \mathrm{C}, 8 \mathrm{~h}\end{array}$ & $62 \%$ & $20 \%$ & $<5 \%$ \\
\hline $\begin{array}{c}\text { p-Toll (40 mol\%), Selectfluor } \\
\text { (2.2 eq.), } \mathrm{CsCl}(6.0 \text { eq.), HFIP } \\
\text { (18.0 eq.), } \mathrm{CH}_{2} \mathrm{Cl}_{2}, 0^{\circ} \mathrm{C}, 8 \mathrm{~h}\end{array}$ & $<5 \%$ & $77 \%$ & $<5 \%$ \\
\hline $\begin{array}{c}\mathrm{Mn}(\mathrm{OTf})_{2}(5 \mathrm{~mol} \%), \mathrm{MgCl}_{2} \\
(3.0 \mathrm{eq} .), \mathrm{LiClO} 4(1.75 \mathrm{eq} .), \\
\mathrm{AcOH} / \mathrm{MeCN}, \mathrm{C}(+) / \mathrm{Pt}(-), \text { Ecell }= \\
2.3 \mathrm{~V}, 40^{\circ} \mathrm{C}^{15}\end{array}$ & $<5 \%$ & $<5 \%$ & $71 \%$ \\
\hline
\end{tabular}

${ }^{1} \mathrm{H}$ NMR yield using DMF as internal standard. 


\section{$N$-allyl-N-(2,3-dichloropropyl)-4-methylbenzenesulfonamide (22)}

$\overbrace{\mathrm{Cl}}^{\mathrm{N}} \widehat{\mathrm{T}}_{\mathrm{S}} \mathrm{N}$-diallyl-4-methylbenzenesulfonamide $\quad(50 \mathrm{mg}, \quad 0.20 \mathrm{mmol}$, 1.0 eq.), $p$-iodotoluene ( $8.7 \mathrm{mg}, 0.04 \mathrm{mmol}, 0.2$ eq.), cesium chloride (101 mg, $0.60 \mathrm{mmol}, 3.0$ eq.) and Selectfluor ${ }^{\circledR}$ (78 mg, $\left.0.22 \mathrm{mmol}, 1.1 \mathrm{eq}.\right)$ were dissolved in a solution of HFIP (302 mg, 1.80 mmol, 9.0 eq.) in $\mathrm{CH}_{2} \mathrm{Cl}_{2}(0.9 \mathrm{~mL})$. The mixture was cooled and stirred at $0^{\circ} \mathrm{C}$ (ice bath). After $8 \mathrm{~h}$, the reaction mixture was quenched with a saturated solution of $\mathrm{Na}_{2} \mathrm{~S}_{2} \mathrm{O}_{3}$ and extracted with $\mathrm{CH}_{2} \mathrm{Cl}_{2}(3 \mathrm{x})$. The combined organic phases were dried over $\mathrm{MgSO}_{4}$, filtered and the solvent was evaporated under vacuo. The residue was dissolved in $0.6 \mathrm{~mL}$ of $\mathrm{CDCl}_{3}$ containing $0.1 \mathrm{mmol}$ of DMF (stock solution) and analysed by ${ }^{1} \mathrm{H}$ NMR (yield 19: $62 \%$, yield 20: $20 \%$ ). The crude mixture was purified by column chromatography using cyclohexane:ethyl acetate $95: 5$ to afford $N$-allyl- $N$-(2,3-dichloropropyl)-4methylbenzenesulfonamide (22) as a white solid (28 $\mathrm{mg}, 0.09 \mathrm{mmol}, 43 \%)$.

Rf: (cyclohexane:ethyl acetate 95:5) 0.12;

M.p. $69-71^{\circ} \mathrm{C}$;

${ }^{1} \mathrm{H}$ NMR $\left(400 \mathrm{MHz}, \mathrm{CDCl}_{3}\right) \delta=7.71(\mathrm{~m}, 2 \mathrm{H}), 7.33(\mathrm{~m}, 2 \mathrm{H}), 5.55\left(\mathrm{ddt}, \mathrm{J}_{\mathrm{HH}}=16.8,10.1\right.$, $6.6 \mathrm{~Hz}, 1 \mathrm{H}), 5.19(\mathrm{~m}, 2 \mathrm{H}), 4.44\left(\mathrm{tt}, \mathrm{J}_{\mathrm{HH}}=7.0,5.0 \mathrm{~Hz}, 1 \mathrm{H}\right), 3.94\left(\mathrm{ddt}, \mathrm{J}_{\mathrm{HH}}=15.5,6.4\right.$, $1.4 \mathrm{~Hz}, 1 \mathrm{H}), 3.86\left(\mathrm{~d}, J_{H H}=5.0 \mathrm{~Hz}, 2 \mathrm{H}\right), 3.82(\mathrm{~m}, 1 \mathrm{H}), 3.56\left(\mathrm{dd}, J_{H H}=15.0,7.3 \mathrm{~Hz}, 1 \mathrm{H}\right)$, $3.27\left(\mathrm{dd}, \mathrm{J}_{\mathrm{HH}}=15.0,6.6 \mathrm{~Hz}, 1 \mathrm{H}\right), 2.44(\mathrm{~s}, 3 \mathrm{H}) \mathrm{ppm}$;

HRMS (ESI) $\mathrm{m} / \mathrm{z}$ [M+Na] ${ }^{+}$calcd. for $\mathrm{C}_{13} \mathrm{H}_{17} \mathrm{Cl}_{2} \mathrm{NNaO}_{2} \mathrm{~S}^{+}: 344.0249$, found: 344.0265 .

The analytic data are in agreement with the literature. ${ }^{15}$ 


\section{$N, N$-bis(2,3-dichloropropyl)-4-methylbenzenesulfonamide (23)}

$\overbrace{\mathrm{Cl}}^{\mathrm{N}} \overbrace{\mathrm{Cl}} \mathrm{Cl} \mathrm{N}, \mathrm{N}$-diallyl-4-methylbenzenesulfonamide $(50 \mathrm{mg}, 0.20 \mathrm{mmol}$, 1.0 eq.), $p$-iodotoluene ( $17 \mathrm{mg}, 0.08 \mathrm{mmol}, 0.4$ eq.), cesium chloride (202 mg, $1.20 \mathrm{mmol}, 6.0$ eq.) and Selectfluor ${ }^{\circledR}(156 \mathrm{mg}, 0.44 \mathrm{mmol}, 2.2$ eq.) were dissolved in a solution of HFIP (604 mg, $3.60 \mathrm{mmol}, 18.0$ eq.) in $\mathrm{CH}_{2} \mathrm{Cl}_{2}(1.8 \mathrm{~mL})$. The mixture was cooled and stirred at $0^{\circ} \mathrm{C}$ (ice bath). After $8 \mathrm{~h}$, the reaction mixture was quenched with a saturated solution of $\mathrm{Na}_{2} \mathrm{~S}_{2} \mathrm{O}_{3}$ and extracted with $\mathrm{CH}_{2} \mathrm{Cl}_{2}(3 \mathrm{x})$. The combined organic phases were dried over $\mathrm{MgSO}_{4}$, filtered and the solvent was evaporated under vacuo. The residue was dissolved in $0.6 \mathrm{~mL}$ of $\mathrm{CDCl}_{3}$ containing $0.1 \mathrm{mmol}$ of DMF (stock solution) and analysed by ${ }^{1} \mathrm{H}$ NMR (yield 20: $77 \%$ ). The crude mixture was purified by column chromatography using cyclohexane:ethyl acetate $9: 1$ to afford N,N-bis(2,3-dichloropropyl)-4-methylbenzenesulfonamide (23) as a colourless oil (49 mg, $0.13 \mathrm{mmol}, 62 \%)$.

Rf: (cyclohexane:ethyl acetate 9:1) 0.21;

${ }^{1} \mathrm{H}$ NMR $\left(400 \mathrm{MHz}, \mathrm{CDCl}_{3}\right) \delta=7.73(\mathrm{~m}, 2 \mathrm{H}), 7.37(\mathrm{~m}, 2 \mathrm{H}), 4.51(\mathrm{~m}, 2 \mathrm{H}), 3.86(\mathrm{~m}, 3 \mathrm{H})$, $3.78(\mathrm{~m}, 3 \mathrm{H}), 3.38\left(\mathrm{dd}, \mathrm{J}_{\mathrm{HH}}=15.3,8.0 \mathrm{~Hz}, 1 \mathrm{H}\right), 3.16\left(\mathrm{dd}, \mathrm{J}_{\mathrm{HH}}=15.1,7.3 \mathrm{~Hz}, 1 \mathrm{H}\right), 2.46$ (s, 3H) ppm;

HRMS (ESI) $\mathrm{m} / z$ [M+Na] ${ }^{+}$calcd. for $\mathrm{C}_{13} \mathrm{H}_{17} \mathrm{Cl}_{4} \mathrm{NNaO}_{2} \mathrm{~S}^{+}: 413.9626$, found: 413.9630 .

The analytic data are in agreement with the literature. ${ }^{15}$ 


\section{c. Hammett correlation}

\section{General Procedure E for kinetic studies on the dichlorination of terminal alkenes generating the Hammett plot}

Hex-5-en-1-yl benzoate (1) (41 mg, $0.20 \mathrm{mmol}, 1.0$ eq.), cesium chloride (101 mg, $0.60 \mathrm{mmol}, 3.0$ eq.) and the iodine catalyst $(10 \mu \mathrm{mol}, 5 \mathrm{~mol} \%)$ were dissolved in $\mathrm{CH}_{2} \mathrm{Cl}_{2}(0.9 \mathrm{~mL})$ and HFIP $(0.1 \mathrm{~mL})$. Selectfluor ${ }^{\circledR}(78.0 \mathrm{mg}, 0.22 \mathrm{mmol}, 1.1$ eq. $)$ was added and the reaction mixture was stirred for $5,10,15$ or $20 \mathrm{~min}$. The reaction mixture was diluted with $\mathrm{CH}_{2} \mathrm{Cl}_{2}$ and a solution of saturated $\mathrm{Na}_{2} \mathrm{~S}_{2} \mathrm{O}_{3}$. The aqueous layer was extracted with $\mathrm{CH}_{2} \mathrm{Cl}_{2}$, dried over $\mathrm{MgSO}_{4}$, filtered and the solvent was removed in vacuo. The NMR yield was obtained by adding DMF $(7.7 \mu \mathrm{L}, 0.10 \mathrm{mmol})$ as internal standard to the crude mixture.

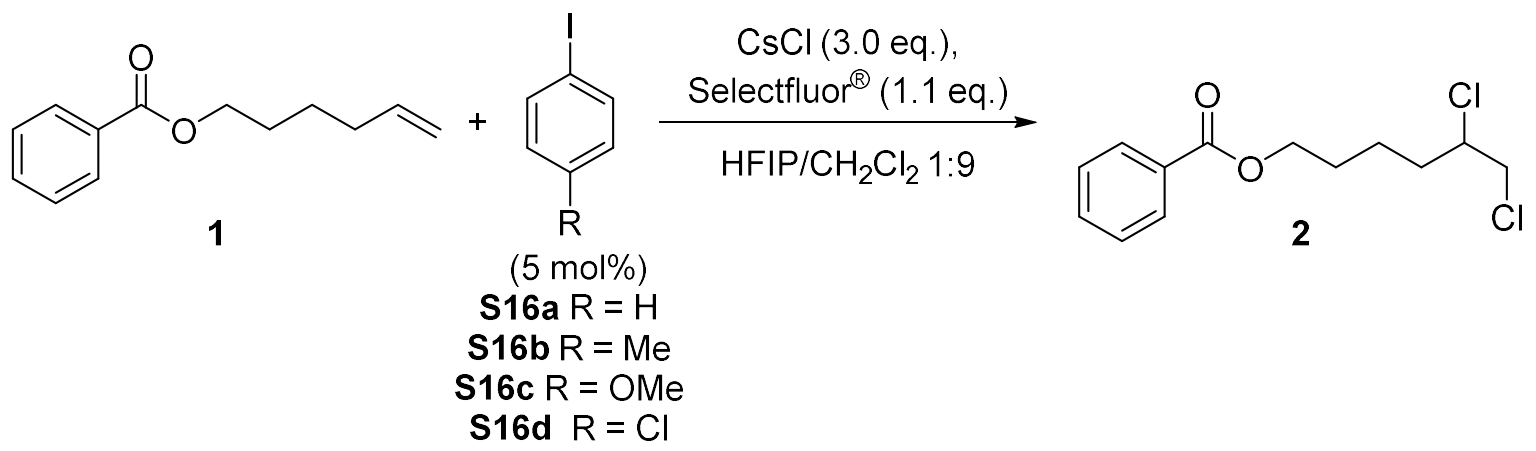

Each experiment was repeated thrice and an average of the obtained yield at each time was formed. The yield was plotted in a diagram versus the time. The slope of the linear equation corresponds to the initial rate constant $k_{R}$ of the reaction. Using the Hammett equation a Hammett plot was generated by plotting the logarithm of the rate constants $k_{R} / k_{H}$ versus the substitution constant $\sigma_{p}$. The slope of the linear equation then corresponds to the reaction constant $\rho$.

$$
\log \left(\mathrm{k}_{R} / k_{H}\right)=\sigma_{\rho} \rho
$$




\section{d. Kinetic studies with 4-iodobenzene (S16a)}

Table S5: ${ }^{1} \mathrm{H}$ NMR yield of the dichlorination of hex-5-en-1-yl benzoate (1) with 4iodobenzene (S16a).

\begin{tabular}{ccccc}
\hline $\begin{array}{c}\text { Time } \\
{[\mathrm{min}]}\end{array}$ & 1. Exp. & 2. Exp. & 3. Exp. & Average \\
\hline 0 & 0 & 0 & 0 & 0 \\
5 & 0 & 1 & 2 & 1 \\
10 & 4 & 7 & 5 & 5 \\
15 & 7 & 7 & 6 & 7 \\
20 & 13 & 20 & 13 & 15 \\
\hline
\end{tabular}

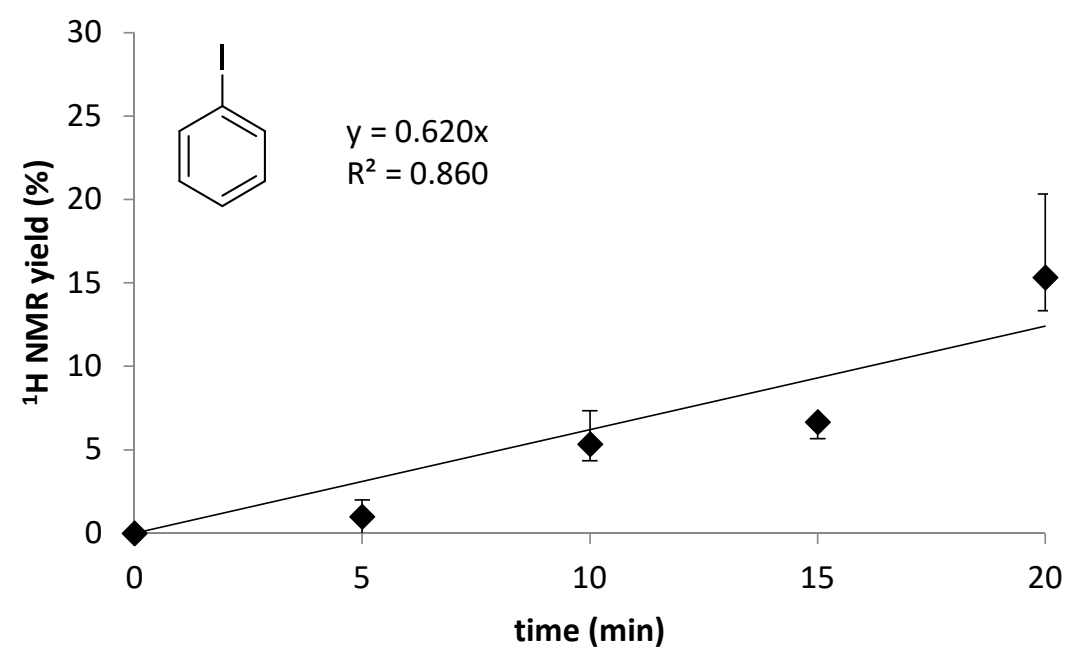

Figure S2: Kinetic data for the dichlorination of hex-5-en-1-yl benzoate (1) with 4iodobenzene (S16a) plotting the ${ }^{1} \mathrm{H}$ NMR yield versus the time. 


\section{e. Kinetic studies with 4-iodotoluene (S16b)}

Table S6: ${ }^{1} \mathrm{H}$ NMR yield of the dichlorination of hex-5-en-1-yl benzoate (1) with 4iodotoluene (S16b).

\begin{tabular}{ccccc}
\hline Time & \multicolumn{4}{c}{${ }^{1} \mathrm{H}$ NMR Yield (\%) } \\
{$[\mathrm{min}]$} & 1. Exp. & 2. Exp. & 3. Exp. & Average \\
\hline 0 & 0 & 0 & 0 & 0 \\
5 & 1 & 4 & 2 & 2 \\
10 & 5 & 8 & 7 & 7 \\
15 & 12 & 10 & 12 & 11 \\
20 & 18 & 25 & 20 & 20 \\
\hline
\end{tabular}

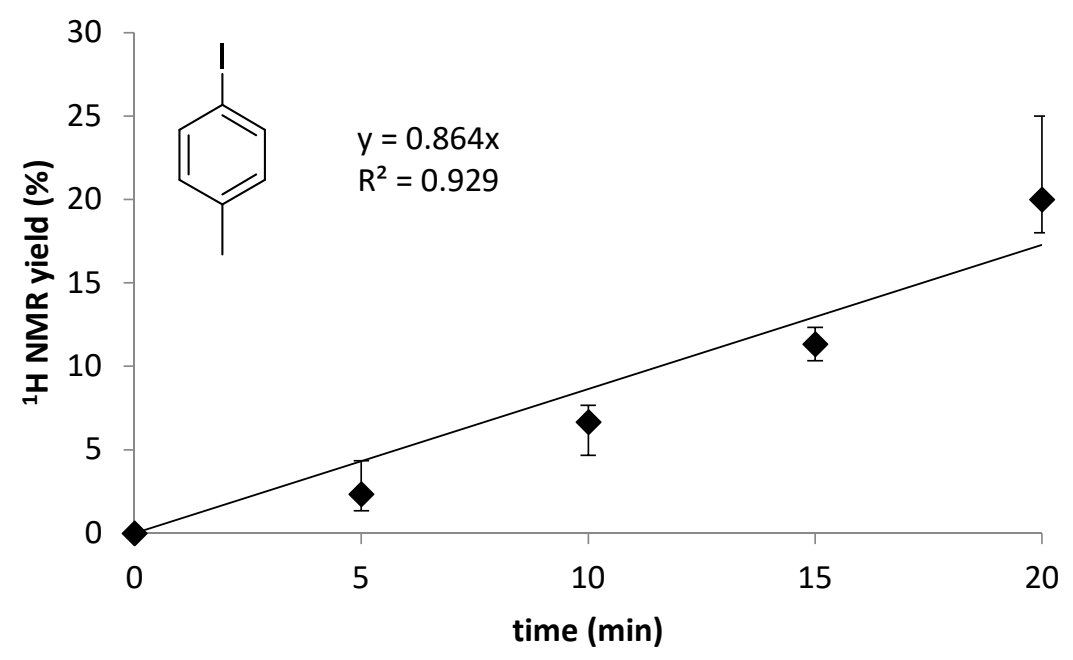

Figure S3: Kinetic data for the dichlorination of hex-5-en-1-yl benzoate (1) with 4iodotoluene (S16b) plotting the ${ }^{1} \mathrm{H}$ NMR yield versus the time. 


\section{f. Kinetic studies with 1-lodo-4-methoxybenzene (S16c)}

Table S7: ${ }^{1} \mathrm{H}$ NMR yield of the dichlorination of hex-5-en-1-yl benzoate (1) with 1iodo-4-methoxybenzene (S16c).

\begin{tabular}{ccccc}
\hline Time & \multicolumn{4}{c}{${ }^{1} \mathrm{H}$ NMR Yield (\%) } \\
{$[\mathrm{min}]$} & 1. Exp. & 2. Exp. & 3. Exp. & Average \\
\hline 0 & 0 & 0 & 0 & 0 \\
5 & 4 & 4 & 4 & 4 \\
10 & 9 & 10 & 16 & 12 \\
15 & 15 & 16 & 16 & 16 \\
20 & 28 & 18 & 25 & 24 \\
\hline
\end{tabular}

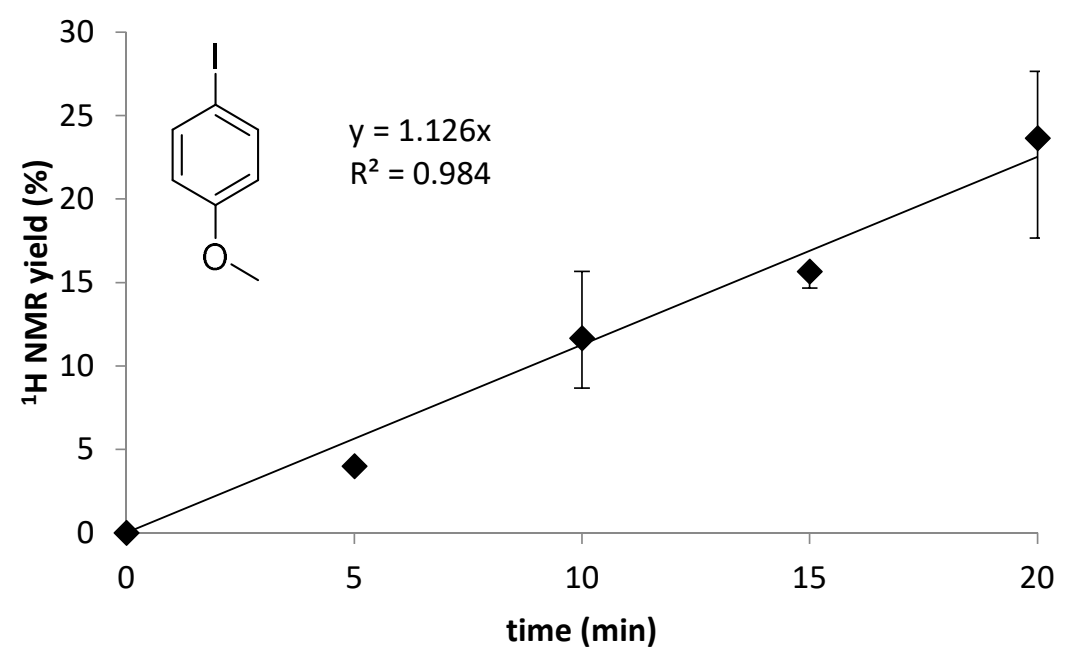

Figure S4: Kinetic data for the dichlorination of hex-5-en-1-yl benzoate (1) with 1iodo-4-methoxybenzene (S16c) plotting the ${ }^{1} \mathrm{H}$ NMR yield versus the time. 


\section{g. Kinetic studies for 1-chloro-4-iodobenzene (S16d)}

Table S8: ${ }^{1} \mathrm{H}$ NMR yield of the dichlorination of hex-5-en-1-yl benzoate (1) with 1chloro-4-iodobenzene (S16d).

\begin{tabular}{ccccc}
\hline Time & \multicolumn{3}{c}{${ }^{1} \mathrm{H}$ NMR Yield (\%) } \\
{$[\mathrm{min}]$} & 1. Exp. & 2. Exp. & 3. Exp. & Average \\
\hline 0 & 0 & 0 & 0 & 0 \\
5 & 0 & 0 & 0 & 0 \\
10 & 2 & 3 & 2 & 2 \\
15 & 4 & 6 & 3 & 4 \\
20 & 12 & 8 & 6 & 9 \\
\hline
\end{tabular}

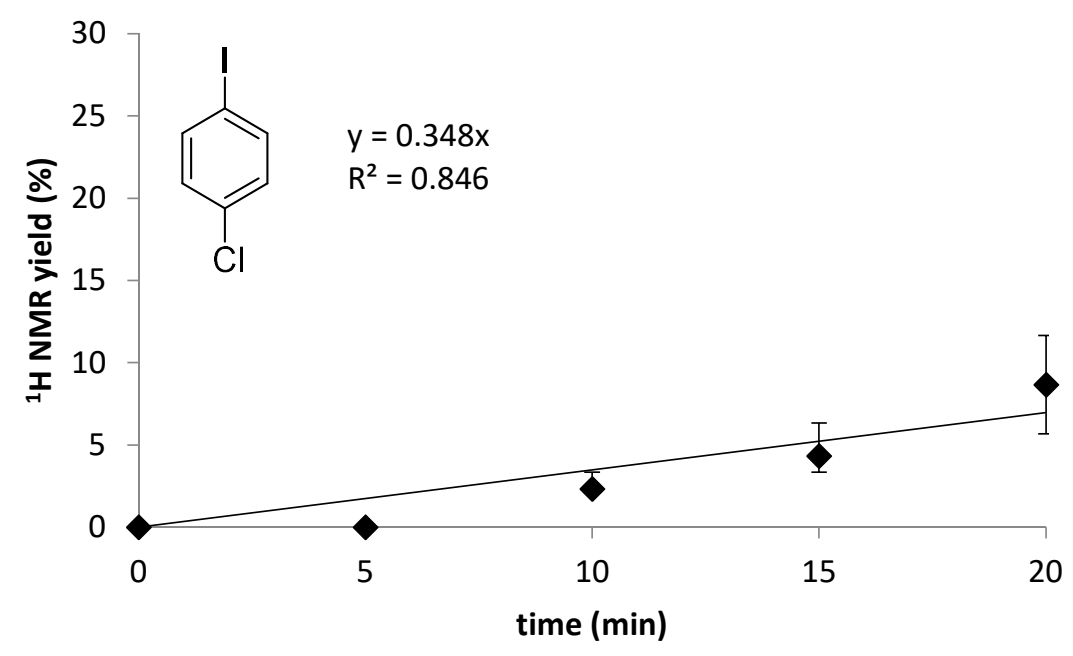

Figure S5: Kinetic data for the dichlorination of hex-5-en-1-yl benzoate (1) with 1chloro-4-iodobenzene (S16d) plotting the ${ }^{1} \mathrm{H}$ NMR yield versus the time. 


\section{h. Kinetic studies: Hammett plot}

Table S9: Kinetic data for the Hammett plot of the dichlorination with different iodoarenes as catalyst.

\begin{tabular}{ccccc}
\hline Substituent & $\sigma_{p}{ }^{61}$ & $k_{R}$ & $k_{R} / k_{H}$ & $\log \left(k_{R} / k_{H}\right)$ \\
\hline OMe & -0.27 & 1.13 & 1.82 & 0.26 \\
$\mathrm{Me}$ & -0.14 & 0.86 & 1.39 & 0.14 \\
$\mathrm{H}$ & 0.00 & 0.62 & 1.00 & 0.00 \\
$\mathrm{Cl}$ & 0.24 & 0.35 & 0.56 & -0.25 \\
\hline
\end{tabular}

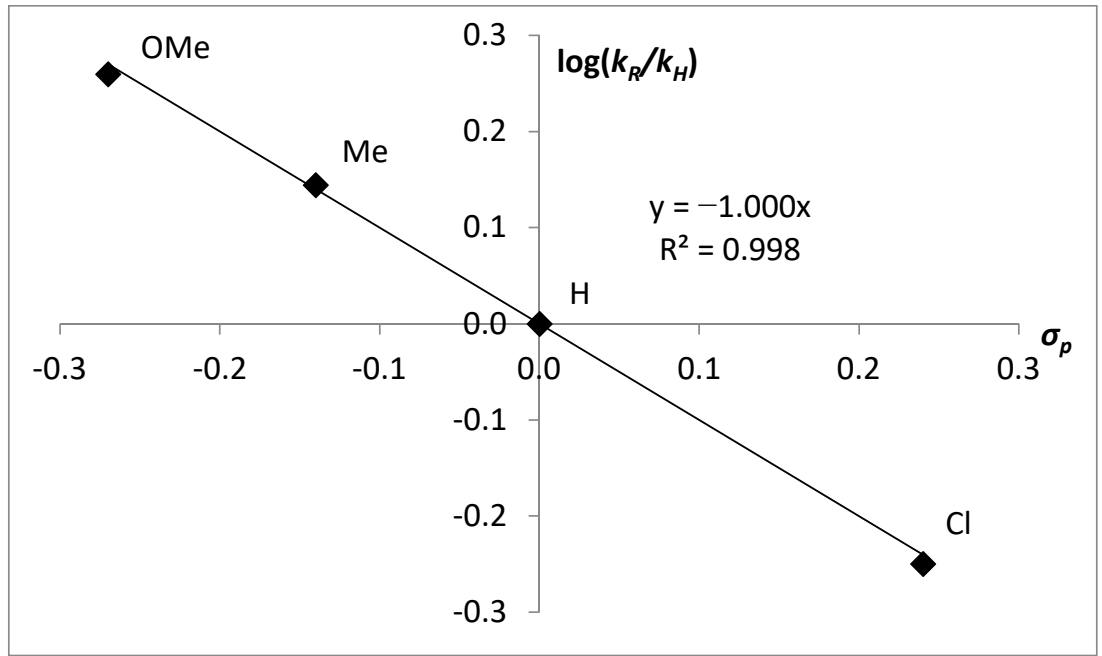

Figure S6: Kinetic data for the Hammett plot of the dichlorination of hex-5-en-1-yl benzoate (1) plotting the logarithm of the initial rate constanst $k$ versus the substituent constants $\sigma_{p}$. 


\section{Preliminary validation of an enantioselective variant}

\section{2-lodo-5-methylbenzene-1,3-diol' ${ }^{19}$ (S17)}<smiles>Cc1cc(O)c(I)c(O)c1</smiles>

This compound was prepared according to a modified procedure from Uchida et al. ${ }^{19}$ 5-Methylbenzene-1,3-diol $(1.24 \mathrm{~g}, 10 \mathrm{mmol}, 1.0$ eq.) was dissolved in acetonitrile $(20 \mathrm{~mL})$ and the resulting solution was cooled to $0^{\circ} \mathrm{C}$. $\mathrm{N}$-iodosuccinimide $(2.25 \mathrm{~g}, 10 \mathrm{mmol}, 1.0$ eq. $)$ was added in one portion and the reaction mixture was stirred at $0^{\circ} \mathrm{C}$ for $15 \mathrm{~min}$. The reaction mixture was quenched with a saturated solution of sodium thiosulfate and extracted with ethyl acetate $(3 x)$. The combined organic layers were washed with water and brine, dried over $\mathrm{Na}_{2} \mathrm{SO}_{4}$, filtered and evaporated under vacuo. The crude mixture was purified by filtration through column chromatography using cyclohexane:ethyl acetate $1: 1$ (15 g of silica) to afford 2-iodo-5-methylbenzene-1,3-diol (S17) as a white solid (2.2 g, $8.8 \mathrm{mmol}, 88 \%)$.

Rf: (cyclohexane:ethyl acetate 1:1) 0.66;

M.p. $101-104^{\circ} \mathrm{C}$;

${ }^{1} \mathrm{H}$ NMR $\left(300 \mathrm{MHz}, \mathrm{CDCl}_{3}\right) \delta=6.40(\mathrm{~m}, 2 \mathrm{H}), 5.20(\mathrm{~s}, 2 \mathrm{H}), 2.26(\mathrm{~s}, 3 \mathrm{H}) \mathrm{ppm}$;

HRMS (ESI) $m / z$ [M-H] $]^{-}$calcd. for $\mathrm{C}_{7} \mathrm{H}_{6} \mathrm{IO}_{2}{ }^{-*}: 248.9418$, found: 248.9443.

The analytic data are in agreement with the literature..$^{20}$

Dimethyl 2,2'-((2-iodo-5-methyl-1,3-phenylene)bis(oxy))(2R,2' $R)$ - dipropionate (S18)<smiles>COC(=O)COc1cc(C)cc(OC(C)C(=O)OC)c1I</smiles>

This compound was prepared according to a modified procedure from Fujita et al. ${ }^{21}$ 2-lodo-5methylbenzene-1,3-diol (1.0 g, $4.0 \mathrm{mmol}, 1.0$ eq.), L- 
methyl lactate (840 $\mu \mathrm{L}, 8.8 \mathrm{mmol}, 2.2$ eq.) and triphenylphosphine $(2.4 \mathrm{~g}, 9.2 \mathrm{mmol}$, 2.3 eq.) were dissolved in tetrahydrofuran $(15 \mathrm{~mL})$ and the resulting solution was cooled to $0^{\circ} \mathrm{C}$. Diisopropyl azodicarboxylate $(1.8 \mathrm{~mL}, 9.2 \mathrm{mmol}, 2.3$ eq.) was added dropwise. The reaction mixture was then allowed to slowly warm up to ambient temperature and was stirred for $16 \mathrm{~h}$. The crude reaction mixture was evaporated under vacuo and directly purified by column chromatography using a gradient cyclohexane:ethyl acetate 95:5 to $9: 1$ to afford dimethyl 2,2'-((2-iodo-5-methyl-1,3phenylene) bis(oxy)) (2R,2'R)- dipropionate (S18) as a light yellow solid (1.4 g, $3.2 \mathrm{mmol}, 81 \%)$.

Rf: (cyclohexane:ethyl acetate 4:1) 0.39;

M.p. $54-56{ }^{\circ} \mathrm{C}$;

ORD $\left(\mathrm{CHCl}_{3}, \mathrm{c} 1.00\right):[\alpha]_{D}^{25}=-2.3^{\circ}$;

${ }^{1} \mathrm{H}$ NMR $\left(300 \mathrm{MHz}, \mathrm{CDCl}_{3}\right) \delta=6.19(\mathrm{~s}, 2 \mathrm{H}), 4.75\left(\mathrm{q}, \mathrm{J}_{\mathrm{HH}}=6.8 \mathrm{~Hz}, 2 \mathrm{H}\right), 3.75(\mathrm{~s}, 6 \mathrm{H})$, $2.25(\mathrm{~s}, 3 \mathrm{H}), 1.69\left(\mathrm{~d}, \mathrm{~J}_{\mathrm{HH}}=6.8 \mathrm{~Hz}, 6 \mathrm{H}\right) \mathrm{ppm}$;

HRMS (ESI) $m / z$ [M+Na] ${ }^{+}$calcd. for $\mathrm{C}_{15} \mathrm{H}_{19} \mathrm{INaO}_{6}{ }^{+}: 445.0119$, found: 445.0133.

The analytic data are in agreement with the literature. ${ }^{20}$

$\left(2 R, 2^{\prime} R\right)-2,2^{\prime}-((2-i o d o-5-m e t h y l-1,3-p h e n y l e n e)$ bis(oxy))dipropionic acid (S19)<smiles>Cc1cc(O[C@H](C)C(=O)O)c(I)c(O[C@H](C)C(=O)O)c1</smiles>
(968 mg, $2.0 \mathrm{mmol}, 1.0$ eq.) was dissolved in tetrahydrofuran (5 mL) and methanol $(5 \mathrm{~mL})$. Then, an aqueous solution of $\mathrm{NaOH} 2 \mathrm{~N}(5 \mathrm{~mL})$ was added to the reaction mixture and the solution was stirred at ambient temperature for $16 \mathrm{~h}$. The reaction 
mixture was treated with an aqueous solution of $\mathrm{HCl} 1 \mathrm{~N}(10 \mathrm{~mL})$ and extracted with ethyl acetate (2x). The combined organic layers were washed with water, brine, dried over $\mathrm{MgSO}_{4}$, filtered and evaporated under vacuo to afford $\left(2 R, 2^{\prime} R\right)-2,2^{\prime}-((2-$ iodo-5methyl-1,3-phenylene)bis(oxy))dipropionic acid (S19) as a white solid (673 mg, $1.7 \mathrm{mmol}, 85 \%)$.

M.p. $148-150{ }^{\circ} \mathrm{C}$;

ORD $\left(\mathrm{CHCl}_{3}, \mathrm{c} 1.00\right):[\alpha]_{D}^{26}=-43.5^{\circ}$;

${ }^{1} \mathrm{H}$ NMR $\left(300 \mathrm{MHz}, \mathrm{DMSO}-d_{6}\right) \delta=13.05(\mathrm{~s}, 2 \mathrm{H}), 6.28(\mathrm{~s}, 2 \mathrm{H}), 4.83\left(\mathrm{q}, \mathrm{J}_{\mathrm{HH}}=6.7 \mathrm{~Hz}\right.$, 2H), $2.21(\mathrm{~s}, 3 \mathrm{H}), 1.53\left(\mathrm{~d}, \mathrm{~J}_{\mathrm{HH}}=6.7 \mathrm{~Hz}, 6 \mathrm{H}\right)$.

HRMS (ESI) m/z [M-H]- calcd. for $\mathrm{C}_{13} \mathrm{H}_{14} \mathrm{IO}_{6}: 392.9841$, found: 392.9864 .

The analytic data are in agreement with the literature. ${ }^{20}$

(Di((3S,5S,7S)-adamantan-1-yl) 2,2'-((2-iodo-5-methyl-1,3-phenylene)bis(oxy)) $\left(2 R, 2^{\prime} R\right)$-dipropionate (S20)

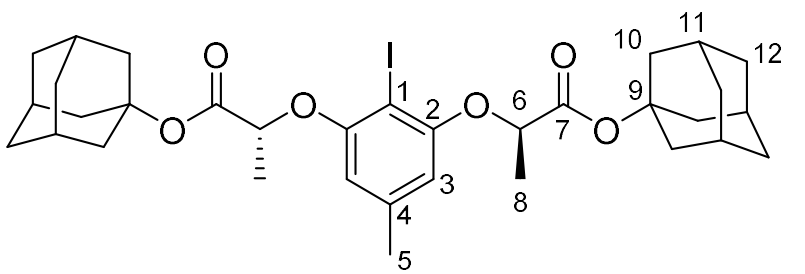

This compound was prepared according to a modified procedure from Uyanik et al. ${ }^{23}\left(2 R, 2^{\prime} R\right)-2,2^{\prime}-((2-$ iodo-5-methyl-1,3phenylene)bis(oxy))dipropionic acid (500 mg, $1.27 \mathrm{mmol}, 1.0$ eq.), 1-adamantanol (487 mg, $3.17 \mathrm{mmol}, 2.5$ eq.) and 4-(dimethylamino)-pyridine were dissolved in $\mathrm{CH}_{2} \mathrm{Cl}_{2}$ $(8 \mathrm{~mL})$ and the resulting solution was cooled down to $0^{\circ} \mathrm{C}$. Then, a solution of $N, N^{\prime}-$ dicyclohexylcarbodiimide (681 mg, $3.30 \mathrm{mmol}, 2.6$ eq.) in $\mathrm{CH}_{2} \mathrm{Cl}_{2}(2 \mathrm{~mL})$ was added dropwise. The reaction mixture was allowed to warm up slowly to ambient temperature and was stirred for $16 \mathrm{~h}$. The reaction mixture was treated with an aqueous solution of $\mathrm{HCl} 1 \mathrm{~N}$ and extracted with $\mathrm{CH}_{2} \mathrm{Cl}_{2}(3 \mathrm{x})$. The combined organic layers were dried over 
$\mathrm{MgSO}_{4}$, filtered and evaporated under vacuo. The crude mixture was purified by column chromatography using cyclohexane:ethyl acetate $95: 5$ to afford (di((3S,5S,7S)adamantan-1-yl) 2,2'-((2-iodo-5-methyl-1,3-phenylene)bis(oxy))-(2R,2'R)-dipropionate (S20) as a white foam (520 $\mathrm{mg}, 0.78 \mathrm{mmol}, 62 \%)$.

Rf: (cyclohexane:ethyl acetate 9:1) 0.47 ;

M.p. $52-56^{\circ} \mathrm{C}$;

ORD $\left(\mathrm{CHCl}_{3}, \mathrm{c} 1.00\right):[\alpha]_{D}^{26}=-8.6^{\circ}$;

${ }^{1} \mathrm{H} \mathrm{NMR}\left(400 \mathrm{MHz}, \mathrm{CDCl}_{3}\right) \delta=6.20\left(\mathrm{~s}, 2 \mathrm{H}, \mathrm{H}^{3}\right), 4.60\left(\mathrm{q},{ }^{3} \mathrm{JHH}_{\mathrm{HH}}=6.8 \mathrm{~Hz}, 2 \mathrm{H}, \mathrm{H}^{6}\right), 2.25$ $\left(\mathrm{s}, 3 \mathrm{H}, \mathrm{H}^{5}\right), 2.15\left(\mathrm{~m}, 6 \mathrm{H}, \mathrm{H}^{11}\right), 2.07\left(\mathrm{~m}, 12 \mathrm{H}, \mathrm{H}^{10}\right), 1.64\left(\mathrm{~d},{ }^{3} \mathrm{~J}_{\mathrm{HH}}=6.7 \mathrm{~Hz}, 6 \mathrm{H}, \mathrm{H}^{8}\right), 1.64$ $\left(\mathrm{m}, 12 \mathrm{H}, \mathrm{H}^{12}\right) \mathrm{ppm}$;

${ }^{13} \mathrm{C}\left\{{ }^{1} \mathrm{H}\right\}$ NMR $\left(101 \mathrm{MHz}, \mathrm{CDCl}_{3}\right) \delta=170.8\left(2 \mathrm{C}, \mathrm{C}^{7}\right), 158.1\left(2 \mathrm{C}, \mathrm{C}^{2}\right), 139.7\left(1 \mathrm{C}, \mathrm{C}^{4}\right)$, $107.9\left(2 \mathrm{C}, \mathrm{C}^{3}\right), 82.0\left(2 \mathrm{C}, \mathrm{C}^{9}\right), 76.5\left(1 \mathrm{C}, \mathrm{C}^{1}\right), 74.5\left(2 \mathrm{C}, \mathrm{C}^{6}\right), 41.3\left(6 \mathrm{C}, \mathrm{C}^{10}\right), 36.2(6 \mathrm{C}$, $\left.C^{12}\right), 31.0\left(6 C, C^{11}\right), 22.0\left(1 C, C^{5}\right), 18.7\left(2 C, C^{8}\right)$.

IR (ATR) $\tilde{v}=2909(\mathrm{~m}), 2852(\mathrm{w}), 1748(\mathrm{~m}), 1726(\mathrm{~m}), 1579(\mathrm{~m}), 1455(\mathrm{~m}), 1418(\mathrm{w})$, $1374(w), 1354(w), 1315(w), 1272(m), 1239(m), 1196(m), 1135(s), 1102(s), 1073$ (s), $1051(s), 1022(m), 966(w), 941(w), 917(w), 897(w), 878(w), 856(w), 812(w)$, $778(w), 754(w), 725(w), 705(w), 663(w) \mathrm{cm}^{-1}$;

HRMS (ESI) $m / z$ [M+Na] $]^{+}$calcd. for $\mathrm{C}_{33} \mathrm{H}_{43} \mid \mathrm{O}_{6} \mathrm{Na}^{+}: 685.1997$, found: 685.1999.

\section{1-Chloro-4-(1,2-dichloroethyl)benzene ${ }^{15}$ (14)}<smiles>ClCC(Cl)c1ccc(Cl)cc1</smiles>
Reaction time of 3 days:

4-Chlorostyrene (28 mg, $0.20 \mathrm{mmol}, 1.0$ eq.), (di((3S,5S,7S)adamantan-1-yl) 2,2'-((2-iodo-5-methyl-1,3-phenylene)bis(oxy))$\left(2 R, 2^{\prime} R\right)$-dipropionate $(27 \mathrm{mg}, 0.04 \mathrm{mmol}, 0.2$ eq.), cesium chloride (101 mg, 
$0.60 \mathrm{mmol}, 3.0$ eq.) and Selectfluor ${ }^{\circledR}(78 \mathrm{mg}, 0.22 \mathrm{mmol}, 1.1$ eq.) were dissolved in a solution of HFIP (302 mg, $1.80 \mathrm{mmol}, 9.0$ eq.) in $\mathrm{CH}_{2} \mathrm{Cl}_{2}(0.9 \mathrm{~mL})$. The mixture was cooled to $-40^{\circ} \mathrm{C}$ and stirred for 3 days using a mechanical stirring. The reaction mixture was then quenched with a saturated solution of $\mathrm{Na}_{2} \mathrm{~S}_{2} \mathrm{O}_{3}$ and extracted with $\mathrm{CH}_{2} \mathrm{Cl}_{2}$ (3x). The combined organic layers were dried over $\mathrm{MgSO}_{4}$, filtered and the solvent was evaporated under vacuo. The e.r. of the product was determined by HPLC analysis using a Daicel Chiralcel OJ-H $\left(0.46 \mathrm{~cm}{ }^{*} 25 \mathrm{~cm}\right)$ column and $n$-hexane:i-propanol $(99.5: 0.5,0.5 \mathrm{~mL} / \mathrm{min})$ as the eluent. The detection was at $230 \mathrm{~nm} . \mathrm{t}_{\mathrm{R}}=17.91 \mathrm{~min}$ (major enantiomer), $t_{R}=17.29$ min (minor enantiomer); e.r.: 64:36. The sample was combined with the crude and evaporated under vacuo. The residue was dissolved in $0.6 \mathrm{~mL}$ of $\mathrm{CDCl}_{3}$ containing $0.1 \mathrm{mmol}$ of DMF (stock solution) and analysed by ${ }^{1} \mathrm{H}$ NMR (yield: $29 \%$ ).

Reaction time of $24 \mathrm{~h}$ :

4-Chlorostyrene (28 mg, 0.20 mmol, 1.0 eq.), (di((3S,5S,7S)-adamantan-1-yl) 2,2'-((2iodo-5-methyl-1,3-phenylene)bis(oxy))-(2R,2'R)-dipropionate $(27 \mathrm{mg}, 0.04 \mathrm{mmol}$, 0.2 eq.), cesium chloride (101 mg, $0.60 \mathrm{mmol}, 3.0$ eq.) and Selectfluor ${ }^{\circledR}(78.0 \mathrm{mg}$, $0.22 \mathrm{mmol}, 1.1$ eq.) were dissolved in a solution of HFIP (302 mg, $1.80 \mathrm{mmol}, 9.0$ eq.) in $\mathrm{CH}_{2} \mathrm{Cl}_{2}(0.9 \mathrm{~mL})$. The mixture was cooled to $-40^{\circ} \mathrm{C}$ and stirred for $24 \mathrm{~h}$ using a mechanical stirring. The reaction mixture was then quenched with a saturated solution of $\mathrm{Na}_{2} \mathrm{~S}_{2} \mathrm{O}_{3}$ and extracted with $\mathrm{CH}_{2} \mathrm{Cl}_{2}(3 \mathrm{x})$. The combined organic layers were dried over $\mathrm{MgSO}_{4}$, filtered and the solvent was evaporated under vacuo. The e.r. of the product was determined by HPLC analysis using a Daicel Chiralcel OJ-H $\left(0.46 \mathrm{~cm}^{*} 25 \mathrm{~cm}\right)$ column and $n$-hexane:i-propanol $(99.5: 0.5,0.5 \mathrm{~mL} / \mathrm{min})$ as the eluent. The detection was at $230 \mathrm{~nm} . \mathrm{t}_{\mathrm{R}}=17.90 \mathrm{~min}$ (major enantiomer), $\mathrm{t}_{\mathrm{R}}=17.36 \mathrm{~min}$ (minor enantiomer), e.r.: 64:36. The sample was combined with the crude and 
evaporated under vacuo. The residue was dissolved in $0.6 \mathrm{~mL}$ of $\mathrm{CDCl}_{3}$ containing $0.1 \mathrm{mmol}$ of DMF (stock solution) and analysed by ${ }^{1} \mathrm{H}$ NMR (yield: $23 \%$ ). The crude was then purified by column chromatography using cyclohexane $100 \%$ to afford 1 chloro-4-(1,2-dichloroethyl)benzene (14) as a colourless oil (6 mg, $0.03 \mathrm{mmol}, 14 \%$ ). The e.r. of the purified product was determined by HPLC analysis using a Daicel Chiralcel OJ-H $\left(0.46 \mathrm{~cm}{ }^{*} 25 \mathrm{~cm}\right)$ column and $n$-hexane : $i$-propanol $(99.5: 0.5$, $0.5 \mathrm{~mL} / \mathrm{min}$ ) as the eluent. The detection was at $230 \mathrm{~nm} . \mathrm{t}_{\mathrm{R}}=18.66 \mathrm{~min}$ (major enantiomer), $t_{R}=18.08 \min ($ minor enantiomer), e.r.: 64:36.

Rf: ( $100 \%$ cyclohexane) 0.44 ;

ORD $\left(\mathrm{CHCl}_{3}, \mathrm{c} 0.50\right):[\alpha]_{D}^{26}=+14.5^{\circ}$ for an e.r. $64: 36$;

${ }^{1} \mathrm{H}$ NMR $\left(300 \mathrm{MHz}, \mathrm{CDCl}_{3}\right) \delta=7.36(\mathrm{~m}, 4 \mathrm{H}), 4.97\left(\mathrm{dd},{ }^{3} \mathrm{HHH}_{\mathrm{HH}}=8.4,6.2 \mathrm{~Hz}, 1 \mathrm{H}\right), 3.99$ $\left(\mathrm{dd},{ }^{3} \mathrm{~J}_{\mathrm{HH}}=11.3,6.2 \mathrm{~Hz}, 1 \mathrm{H}\right), 3.88\left(\mathrm{dd},{ }^{3} \mathrm{~J}_{\mathrm{HH}}=11.4,8.4 \mathrm{~Hz}, 1 \mathrm{H}\right)$.

MS (EI) $m / z(\%)=208.0(12), 210.0(12), 212.0(4)[\mathrm{M}]^{+}, 173.0(26)[\mathrm{M}-\mathrm{Cl}]^{+}, 159.0(100)$ $\left[\mathrm{M}-\mathrm{CH}_{2} \mathrm{Cl}\right]^{+}$.

The analytic data are in agreement with the literature. ${ }^{15}$ 


\section{HPLC traces}

\section{1-Chloro-4-(1,2-dichloroethyl)benzene (14)}

HPLC trace: racemic sample.

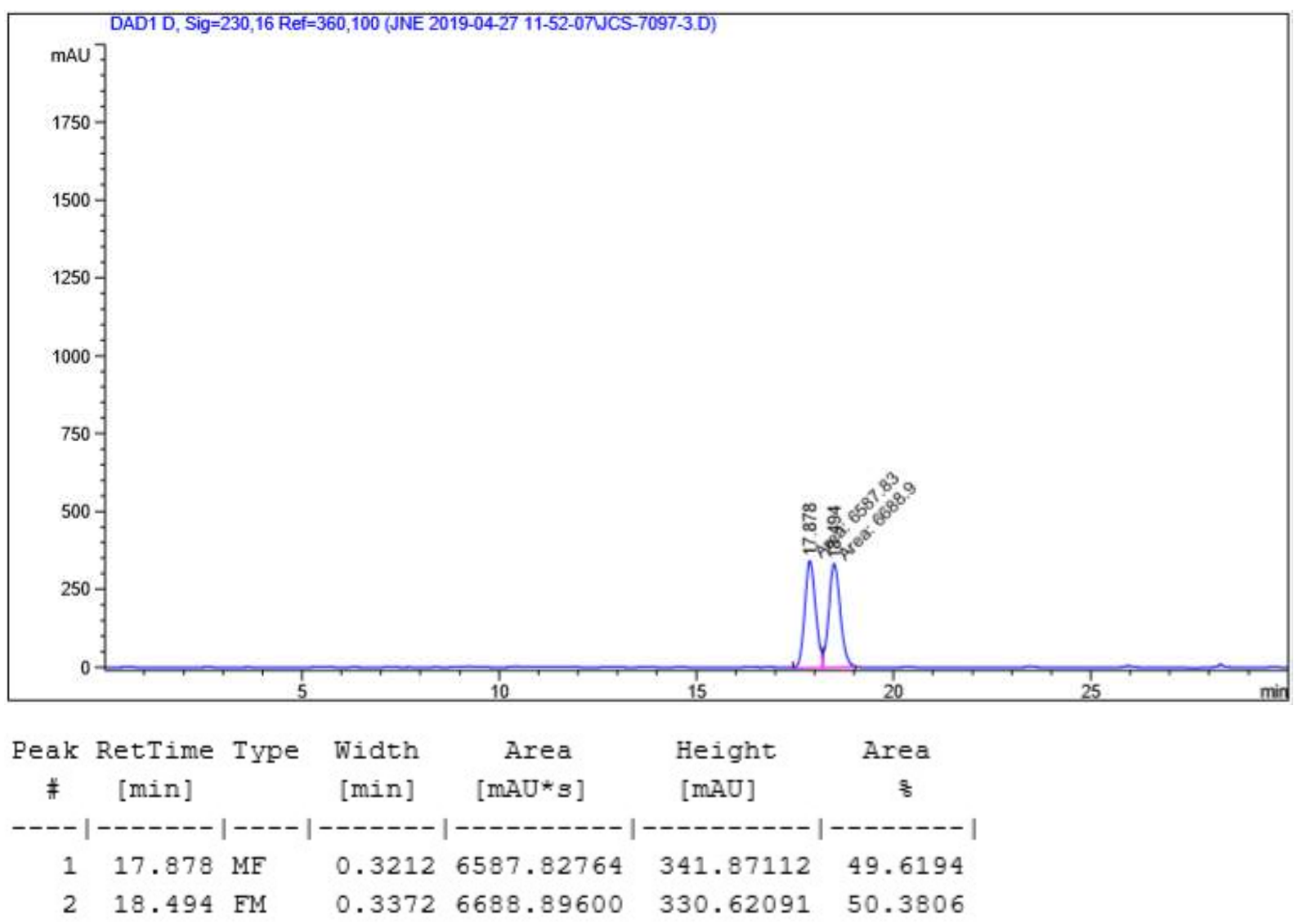


HPLC trace: crude reaction mixture using catalyst $\mathbf{S 2 0}$ after a reaction time of $24 \mathrm{~h}$.

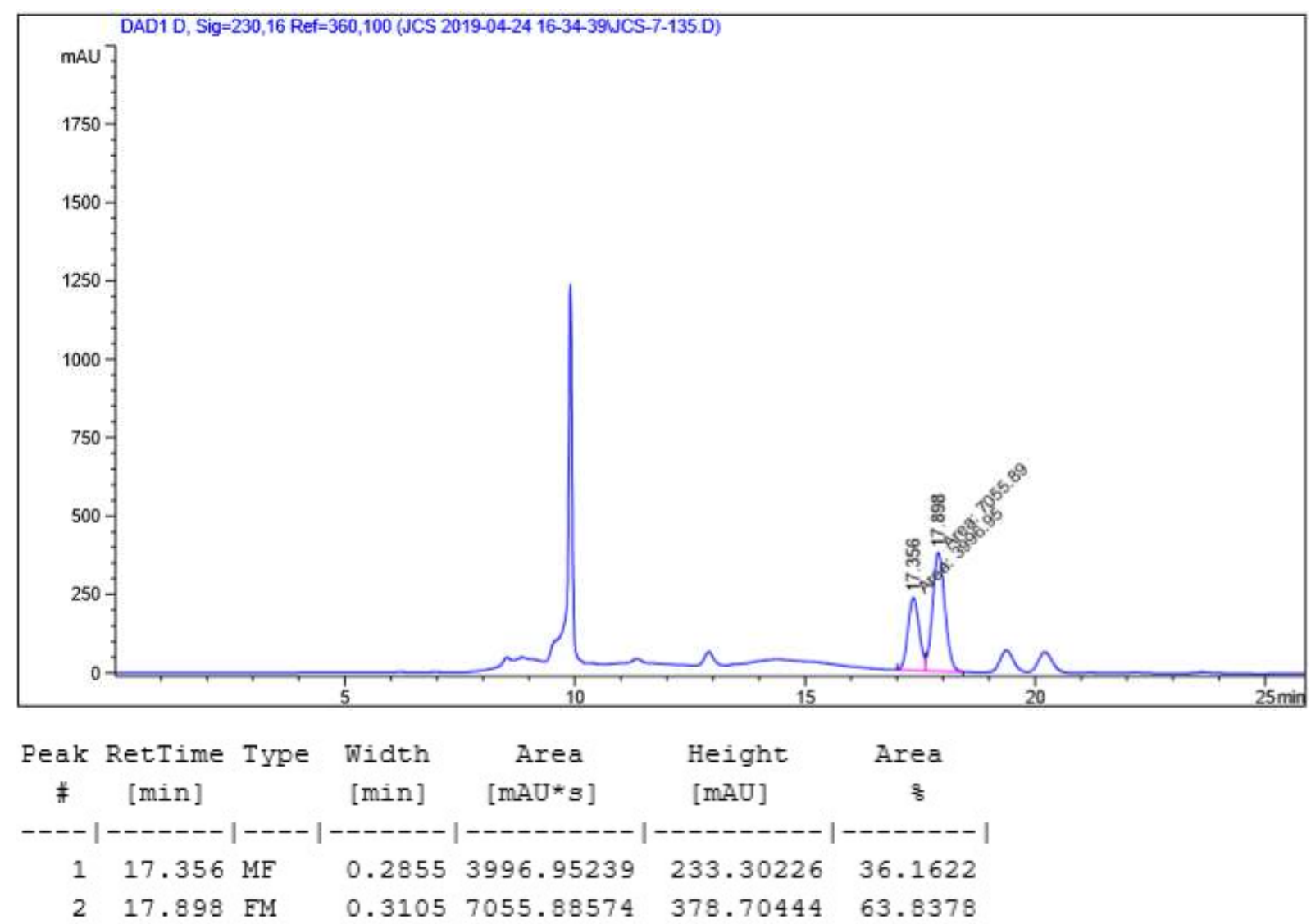

HPLC trace: product 23 after column chromatography.

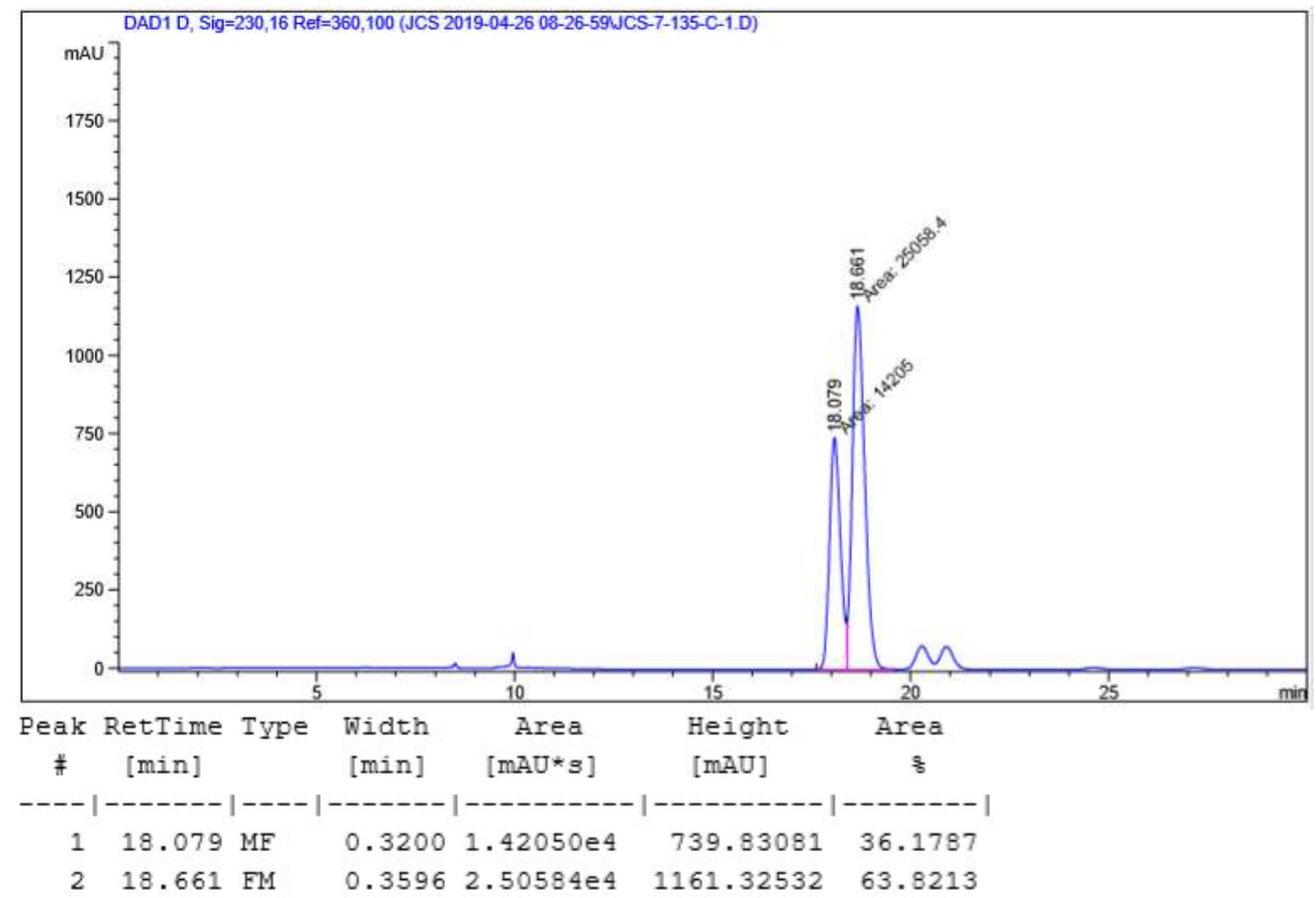


HPLC trace: crude reaction mixture using catalyst $\mathbf{S 2 0}$ after a reaction time of 3 days.

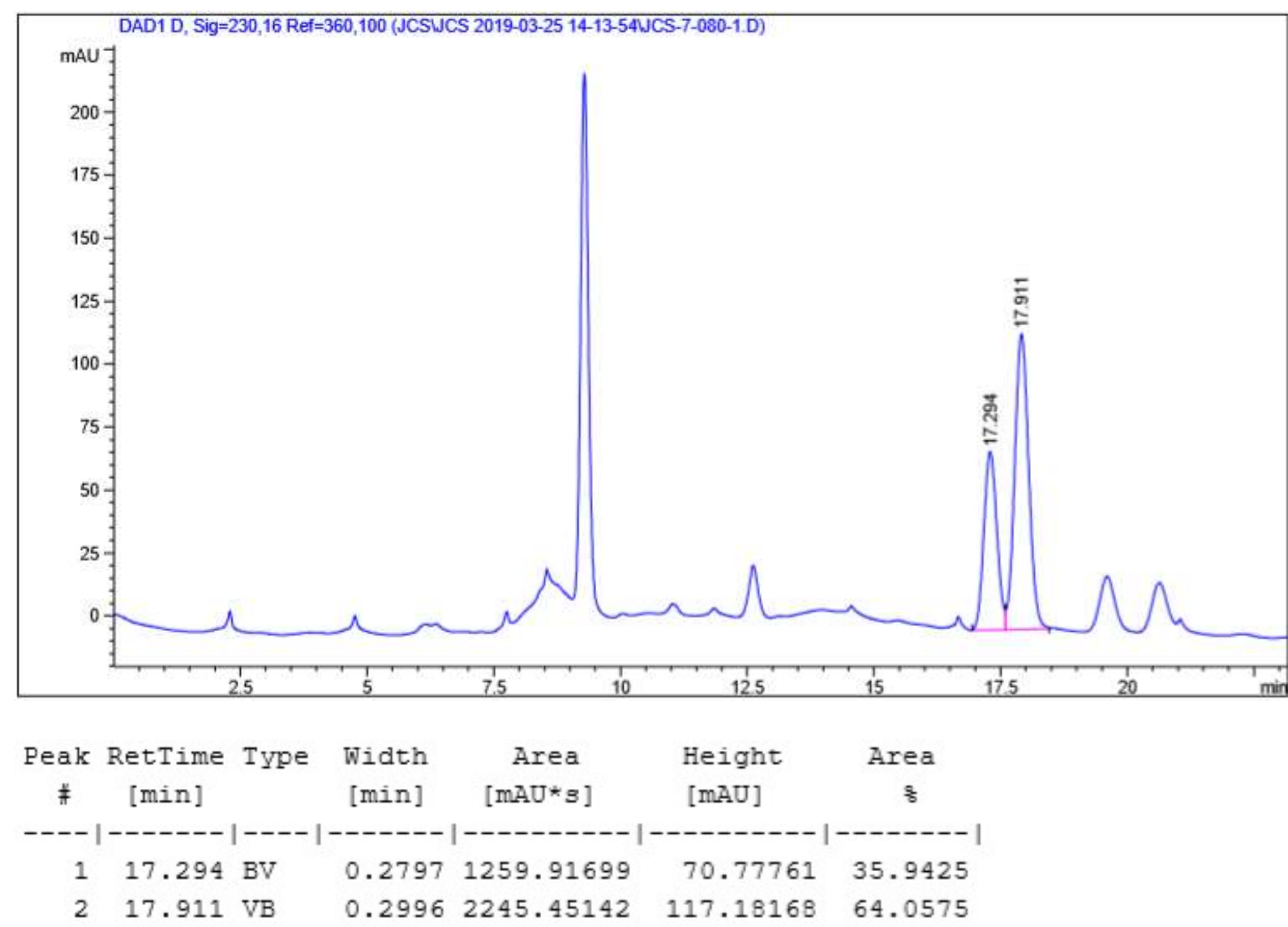




\section{X-Ray Crystallographic Data}

X-Ray diffraction: Data sets were collected with a D8 Venture Dual Source 100 CMOS diffractometer. Programs used: data collection: APEX3 V2016.1-0 (Bruker AXS Inc., 2016); cell refinement: SAINT V8.37A (Bruker AXS Inc., 2015); data reduction: SAINT V8.37A (Bruker AXS Inc., 2015); absorption correction, SADABS V2014/7 (Bruker AXS Inc., 2014); structure solution SHELXT-2015 (Sheldrick, G. M. Acta Cryst., 2015, A71, 3-8); structure refinement SHELXL-2015 (Sheldrick, G. M. Acta Cryst., 2015, C71 (1), 3-8) and graphics, XP (Version 5.1, Bruker AXS Inc., Madison, Wisconsin, USA, 1998). $R$-values are given for observed reflections, and $w R^{2}$ values are given for all reflections.

Exceptions and special features: For compound 19 the chlorinated alkyl chain was found disordered over two positions in the asymmetric unit. Several restraints (SADI, SAME, ISOR, DFIX and SIMU) were used in order to improve refinement stability.

X-ray crystal structure analysis of $p$-Toll(III)Cl $\mathrm{Cl}_{2}$ (S15, gil8779): A pale yellow prismlike specimen of $\mathrm{C}_{7} \mathrm{H}_{7} \mathrm{Cl}_{2}$ l, approximate dimensions $0.065 \mathrm{~mm} \times 0.109 \mathrm{~mm} \times 0.114$ $\mathrm{mm}$, was used for the X-ray crystallographic analysis. The X-ray intensity data were measured. A total of 1138 frames were collected. The total exposure time was 15.81 hours. The frames were integrated with the Bruker SAINT software package using a narrow-frame algorithm. The integration of the data using a monoclinic unit cell yielded a total of 7283 reflections to a maximum $\theta$ angle of $27.54^{\circ}$ ( $0.77 \AA$ resolution), of which 1050 were independent (average redundancy 6.936, completeness $=99.2 \%, R_{\text {int }}=$ $\left.3.35 \%, R_{\text {sig }}=2.12 \%\right)$ and $1026(97.71 \%)$ were greater than $2 \sigma\left(F^{2}\right)$. The final cell constants of $\underline{a}=9.0964(5) \AA, \underline{b}=13.1668(7) \AA, \underline{c}=7.9041(4) \AA, \beta=107.089(2)^{\circ}$, volume $=904.88(8) \AA^{3}$, are based upon the refinement of the XYZ-centroids of 5927 reflections above $20 \sigma(\mathrm{I})$ with $5.614^{\circ}<2 \theta<55.07^{\circ}$. Data were corrected for absorption 
effects using the multi-scan method (SADABS). The ratio of minimum to maximum apparent transmission was 0.812. The calculated minimum and maximum transmission coefficients (based on crystal size) are 0.6550 and 0.7790 . The structure was solved and refined using the Bruker SHELXTL Software Package, using the space group $\mathrm{C} 2 / \mathrm{c}$, with $\mathrm{Z}=4$ for the formula unit, $\mathrm{C}_{7} \mathrm{H}_{7} \mathrm{Cl}_{2} \mathrm{l}$. The final anisotropic full-matrix least-squares refinement on $\mathrm{F}^{2}$ with 49 variables converged at $\mathrm{R} 1=1.76 \%$, for the observed data and $w R 2=4.46 \%$ for all data. The goodness-of-fit was 1.142 . The largest peak in the final difference electron density synthesis was $0.912 \mathrm{e}^{-} / \AA^{3}$ and the largest hole was $-0.972 \mathrm{e}^{-} / \AA^{3}$ with an RMS deviation of $0.107 \mathrm{e}^{-} / \AA^{3}$. On the basis of the final model, the calculated density was $2.121 \mathrm{~g} / \mathrm{cm}^{3}$ and $F(000), 544 \mathrm{e}^{-}$. CCDC number: 1555066.

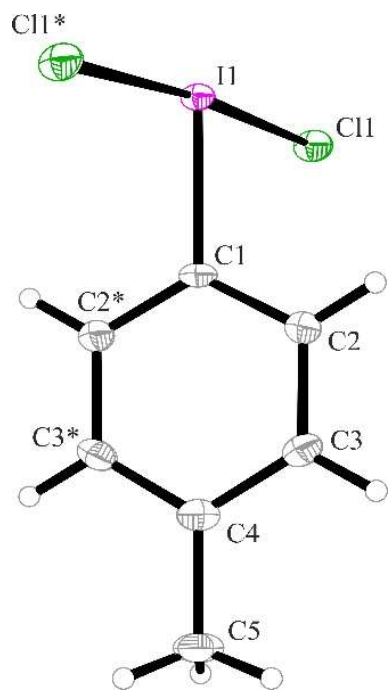

Figure S10: Crystal structure of compound $p$-Toll(III) $\mathrm{Cl}_{2}$ (S15). Thermal ellipsoids are set at $50 \%$ probability. 


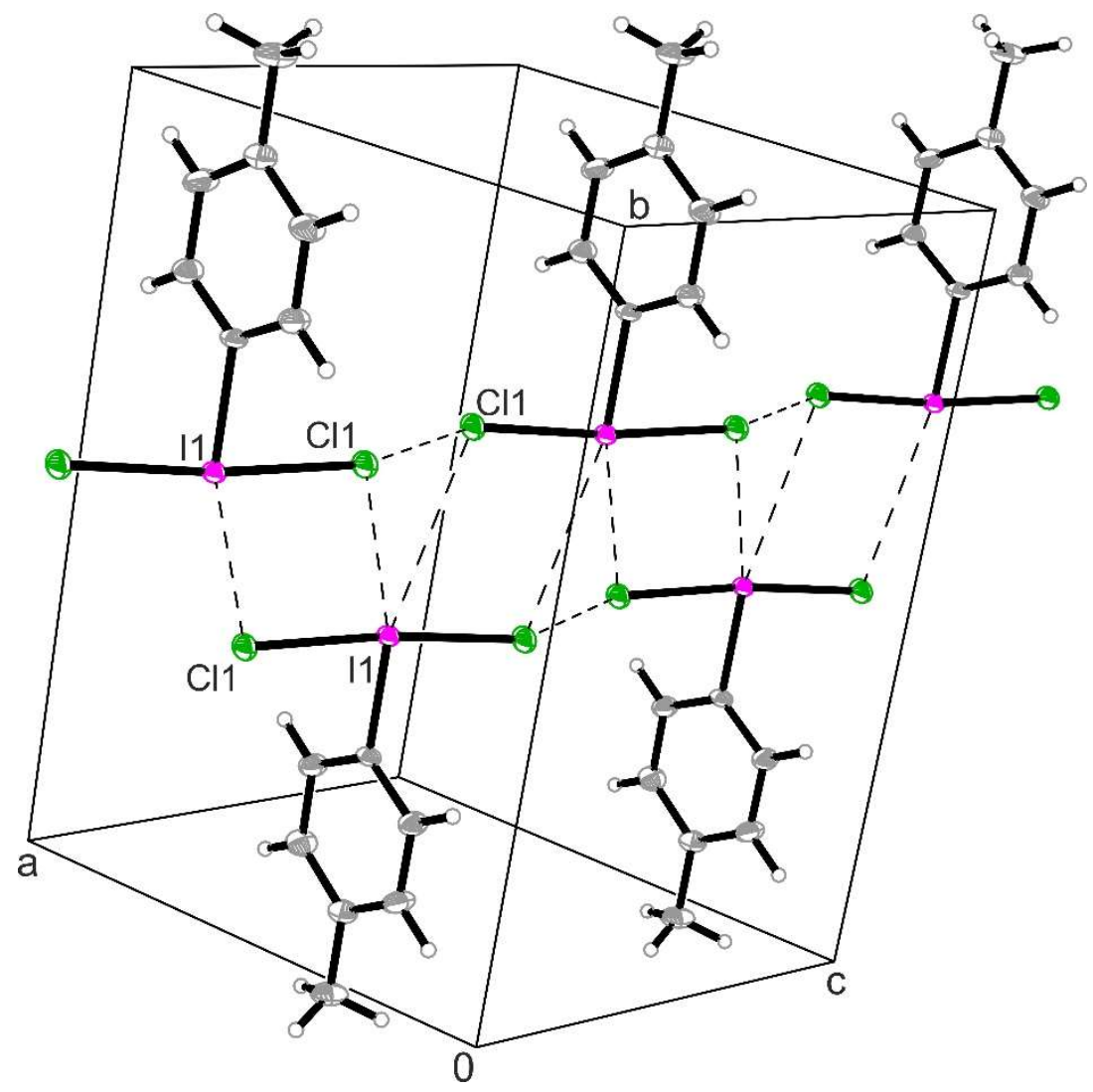

Figure S11: Excerpt of the packing diagram of compound $p$-Toll(III) $\mathrm{Cl}_{2}$ (S15) representing the formation of a supramolecular chain along the ac-diagonal involving non-covalent intermolecular interactions $\mathrm{I} \cdots \mathrm{Cl}$ and $\mathrm{Cl} \cdots \mathrm{Cl}$

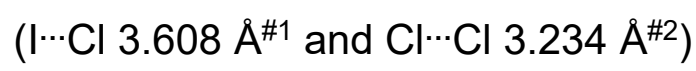

Symmetry transformations used to generate equivalent atoms: ${ }^{\# 1}-x+1,-y+2,-z+1 ;{ }^{\# 2} x,-y+2, z-1 / 2$. 
X-ray crystal structure analysis of 19 (gil9203): A colourless plate-like specimen of $\mathrm{C}_{13} \mathrm{H}_{14} \mathrm{Cl}_{2} \mathrm{~N}_{2} \mathrm{O}_{6}$, approximate dimensions $0.051 \mathrm{~mm} \times 0.122 \mathrm{~mm} \times 0.208 \mathrm{~mm}$, was used for the X-ray crystallographic analysis. The X-ray intensity data were measured. A total of 1254 frames were collected. The total exposure time was 21.44 hours. The frames were integrated with the Bruker SAINT software package using a wide-frame algorithm. The integration of the data using a monoclinic unit cell yielded a total of 18112 reflections to a maximum $\theta$ angle of $68.35^{\circ}$ ( $0.83 \AA$ resolution), of which 2869 were independent (average redundancy 6.313 , completeness $=99.6 \%, \mathrm{R}_{\mathrm{int}}=5.68 \%$, $\left.R_{\text {sig }}=3.99 \%\right)$ and $2419(84.32 \%)$ were greater than $2 \sigma\left(F^{2}\right)$. The final cell constants of $\underline{\mathrm{a}}=14.7104(4) \AA, \underline{\mathrm{b}}=6.5032(2) \AA, \underline{\mathrm{c}}=17.3354(5) \AA, \beta=109.6840(10)^{\circ}$, volume $=$ $1561.48(8) \AA^{3}$, are based upon the refinement of the $X Y Z$-centroids of 7806 reflections above $20 \sigma(\mathrm{I})$ with $10.56^{\circ}<2 \theta<136.6^{\circ}$. Data were corrected for absorption effects using the multi-scan method (SADABS). The ratio of minimum to maximum apparent transmission was 0.846. The calculated minimum and maximum transmission coefficients (based on crystal size) are 0.4860 and 0.8200 . The structure was solved and refined using the Bruker SHELXTL Software Package, using the space group $P 21 / c$, with $Z=4$ for the formula unit, $\mathrm{C}_{13} \mathrm{H}_{14} \mathrm{Cl}_{2} \mathrm{~N}_{2} \mathrm{O}_{6}$. The final anisotropic full-matrix least-squares refinement on $\mathrm{F}^{2}$ with 290 variables converged at $\mathrm{R} 1=4.94 \%$, for the observed data and $w R 2=12.23 \%$ for all data. The goodness-of-fit was 1.041 . The largest peak in the final difference electron density synthesis was $0.272 \mathrm{e}^{-} / \AA^{3}$ and the largest hole was $-0.401 \mathrm{e} / \AA^{3}$ with an RMS deviation of $0.049 \mathrm{e} / \AA^{3}$. On the basis of the final model, the calculated density was $1.553 \mathrm{~g} / \mathrm{cm}^{3}$ and $F(000), 752 \mathrm{e}-$. CCDC number: 1905463. 


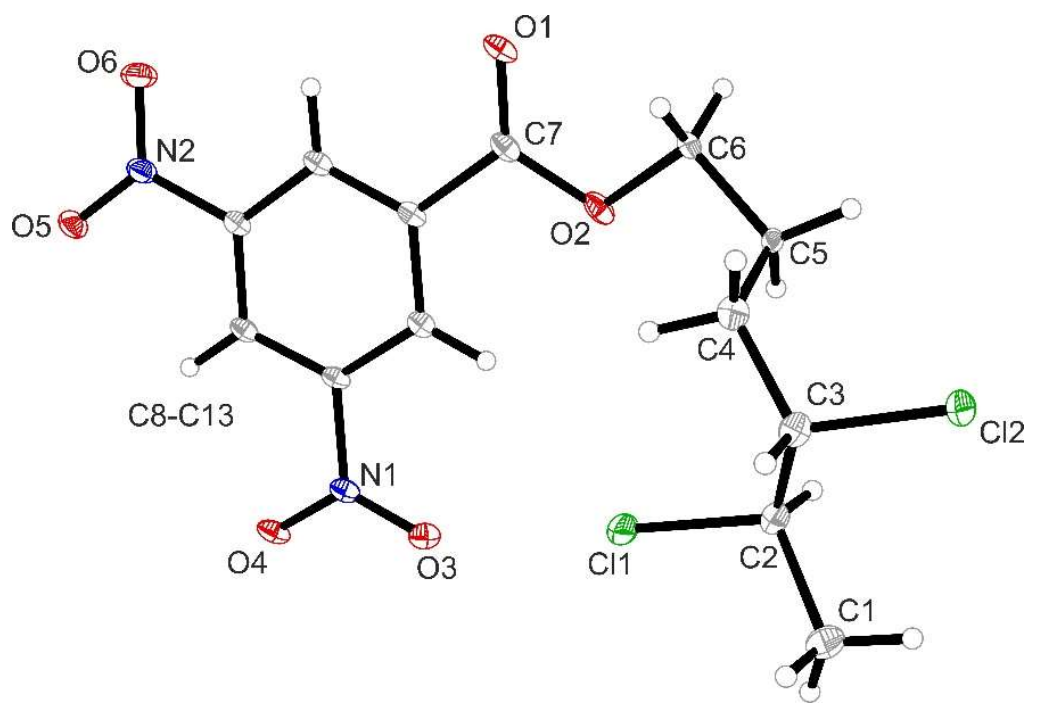

Figure S12: Crystal structure of compound 19.

Thermal ellipsoids are set at $15 \%$ probability.

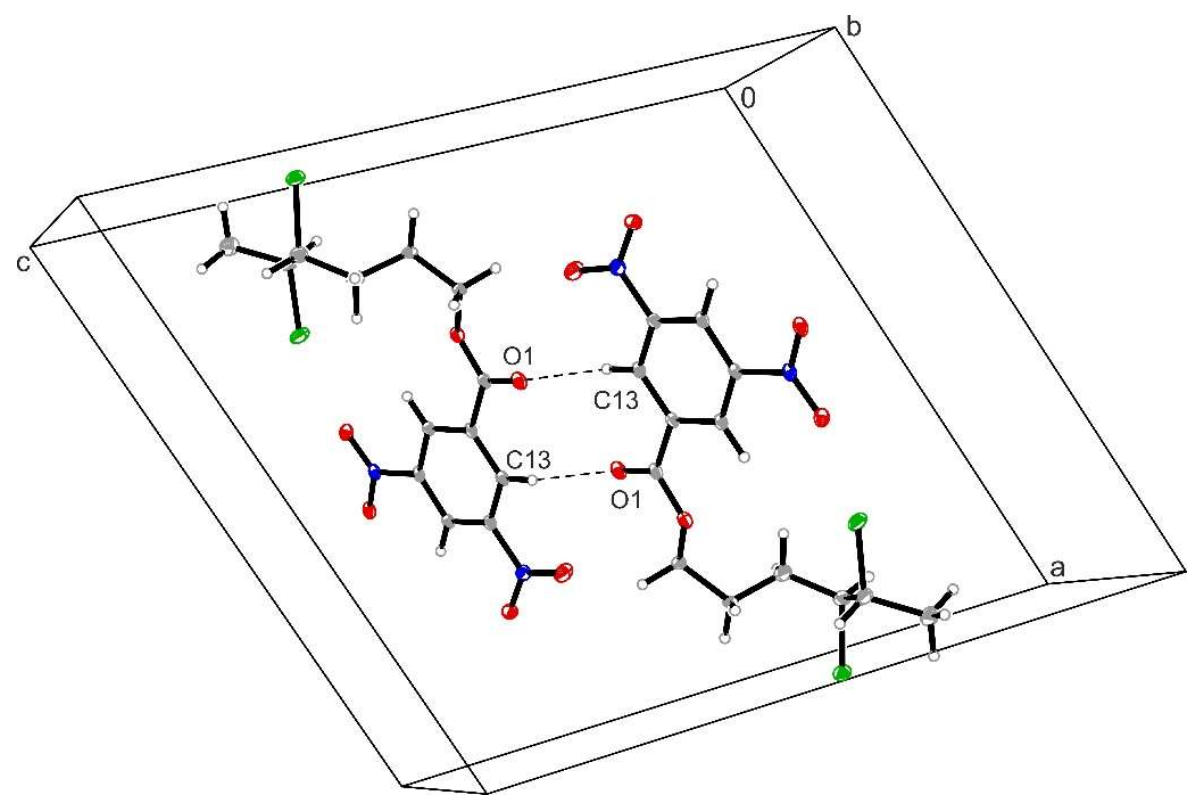

Figure S13: Dimer formation involving $\mathrm{C}-\mathrm{H} \cdots \mathrm{O}$ interaction in compound 19. 


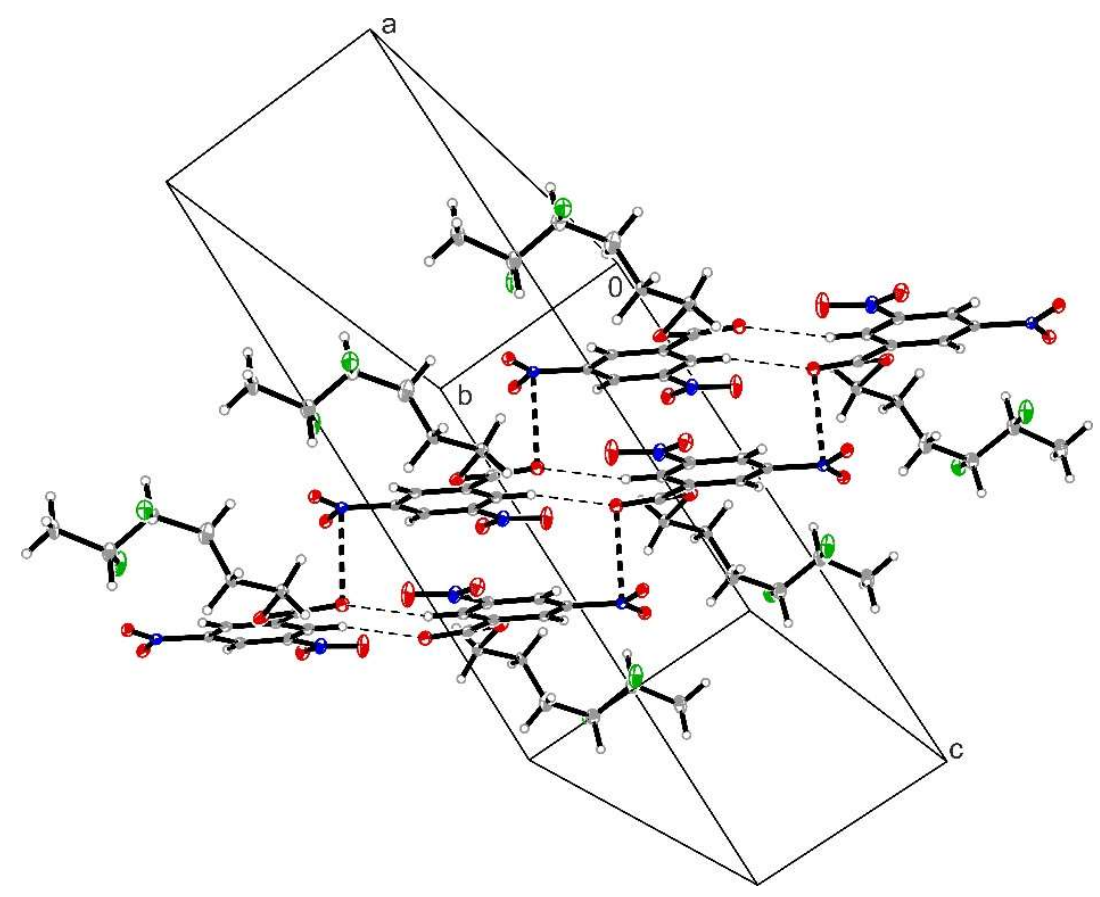

Figure S14: Excerpt of the packing diagram of compound 19 representing the formation of a linear stairs chain along the $b$-axis trough non-covalent intermolecular $\pi \cdots \pi$ interactions

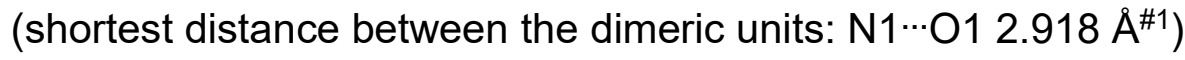

Symmetry transformations used to generate equivalent atoms: ${ }^{\# 1} x, y+1, z$.

1. APEX3 (2016), SAINT (2015) and SADABS (2015), Bruker AXS Inc., Madison, Wisconsin, USA.

2. Sheldrick, G. M., SHELXT - Integrated space-group and crystal-structure determination, Acta Cryst., 2015, A71, 3-8.

3. Sheldrick, G.M., Crystal structure refinement with SHELXL, Acta Cryst., 2015, C71 (1), 3-8.

4. XP - Interactive molecular graphics, Version 5.1, Bruker AXS Inc., Madison, Wisconsin, USA, 1998. 
X. NMR Spectra of unreported starting materials

(E)-Hex-4-en-1-yl 4-methoxybenzoate (S3)

${ }^{1} \mathrm{H} \mathrm{NMR}\left(600 \mathrm{MHz}, \mathrm{CDCl}_{3}\right)$

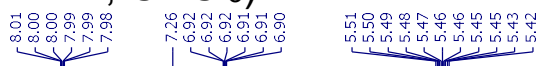

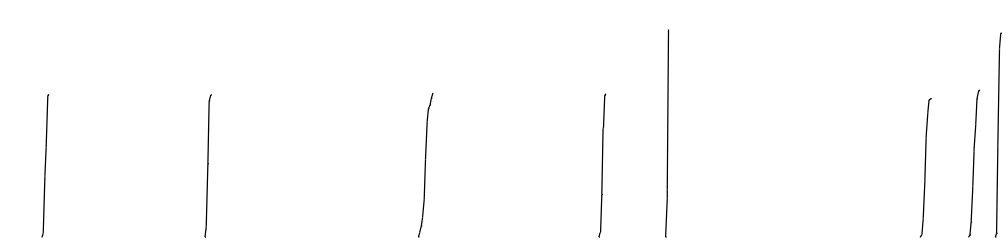<smiles>C/C=C/CCCOC(=O)c1ccc(OC)cc1</smiles>

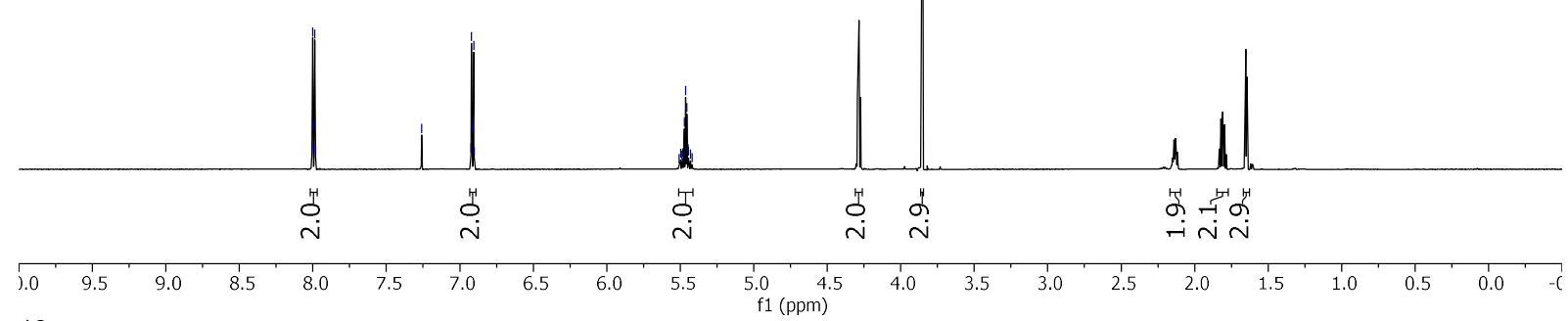

${ }^{13} \mathrm{C} \mathrm{NMR}\left(151 \mathrm{MHz}, \mathrm{CDCl}_{3}\right)$

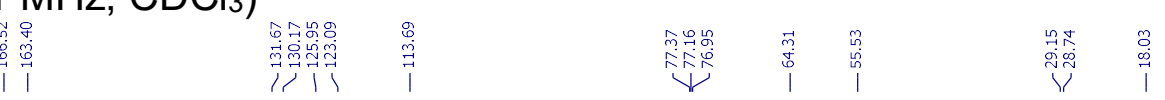<smiles>C/C=C/CCCOC(=O)c1ccc(OC)cc1</smiles>

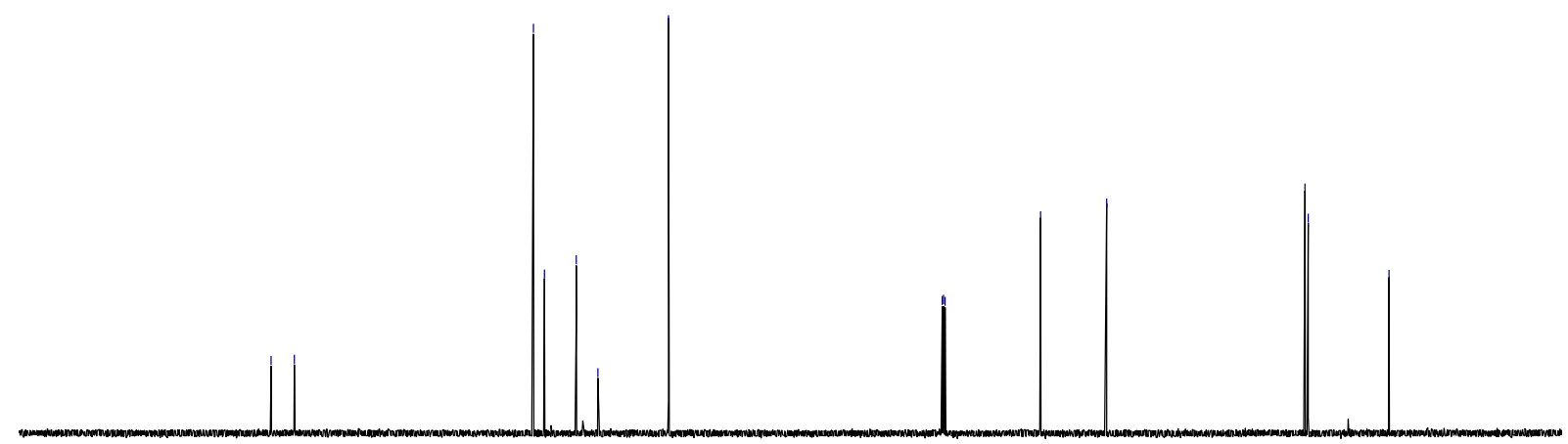

$\begin{array}{lllllllllllllllllllll}190 & 180 & 170 & 160 & 150 & 140 & 130 & 120 & 110 & \begin{array}{c}100 \\ f 1(\mathrm{ppm})\end{array} & 90 & 80 & 70 & 60 & 50 & 40 & 30 & 20 & 10 & 0\end{array}$ 
(E)-Hex-4-en-1-yl 3,5-dinitrobenzoate (S4)

${ }^{1} \mathrm{H}$ NMR (400 MHz, $\left.\mathrm{CDCl}_{3}\right)$

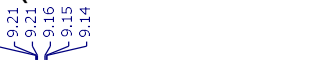

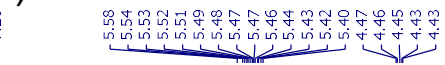

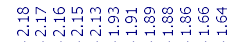

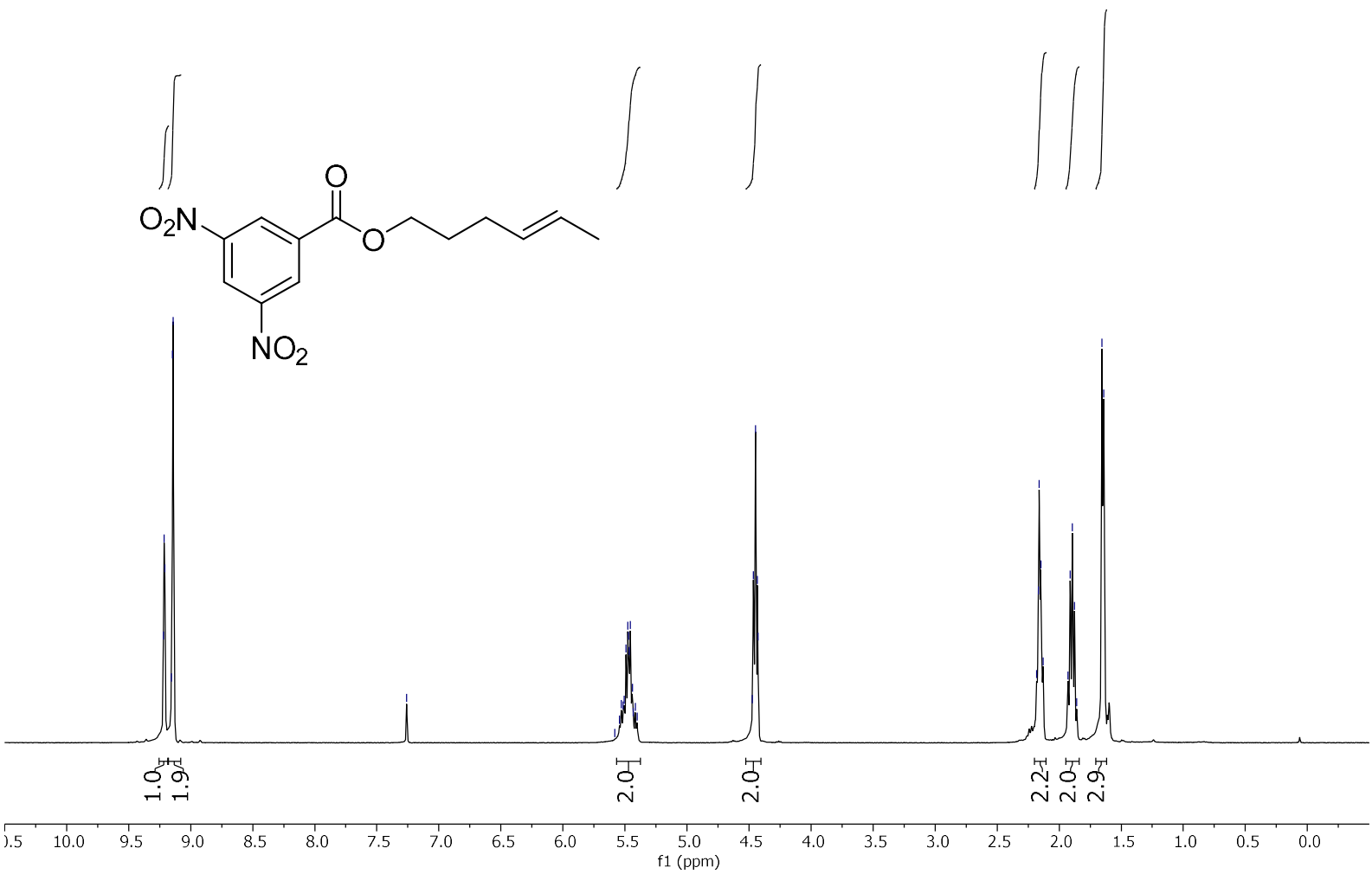

${ }^{13} \mathrm{C}$ NMR $\left(101 \mathrm{MHz}, \mathrm{CDCl}_{3}\right)$

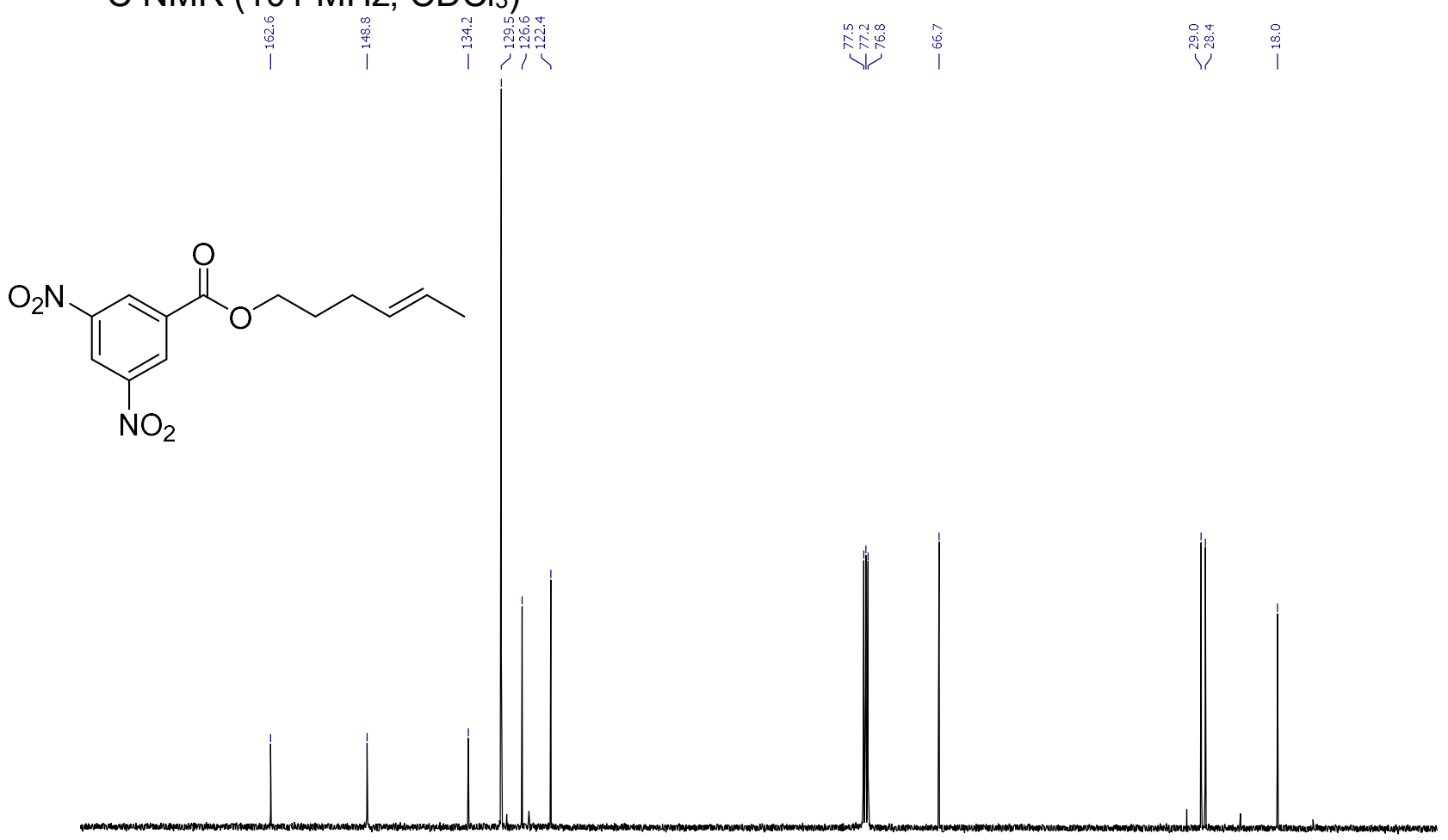

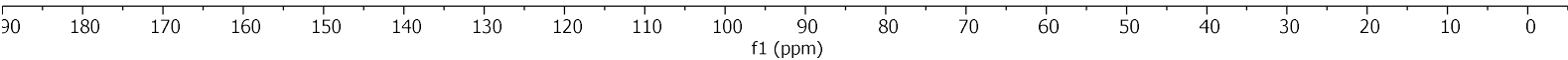


(Z)-Hex-4-en-1-yl 3,5-dinitrobenzoate (S5)

${ }^{1} \mathrm{H}$ NMR $\left(400 \mathrm{MHz}, \mathrm{CDCl}_{3}\right)$

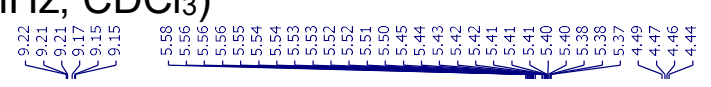

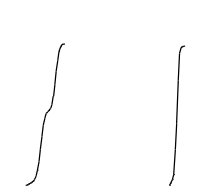

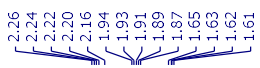

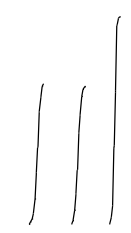<smiles>C/C=C\CCCOC(=O)c1cc([N+](=O)[O-])cc([N+](=O)[O-])c1</smiles>

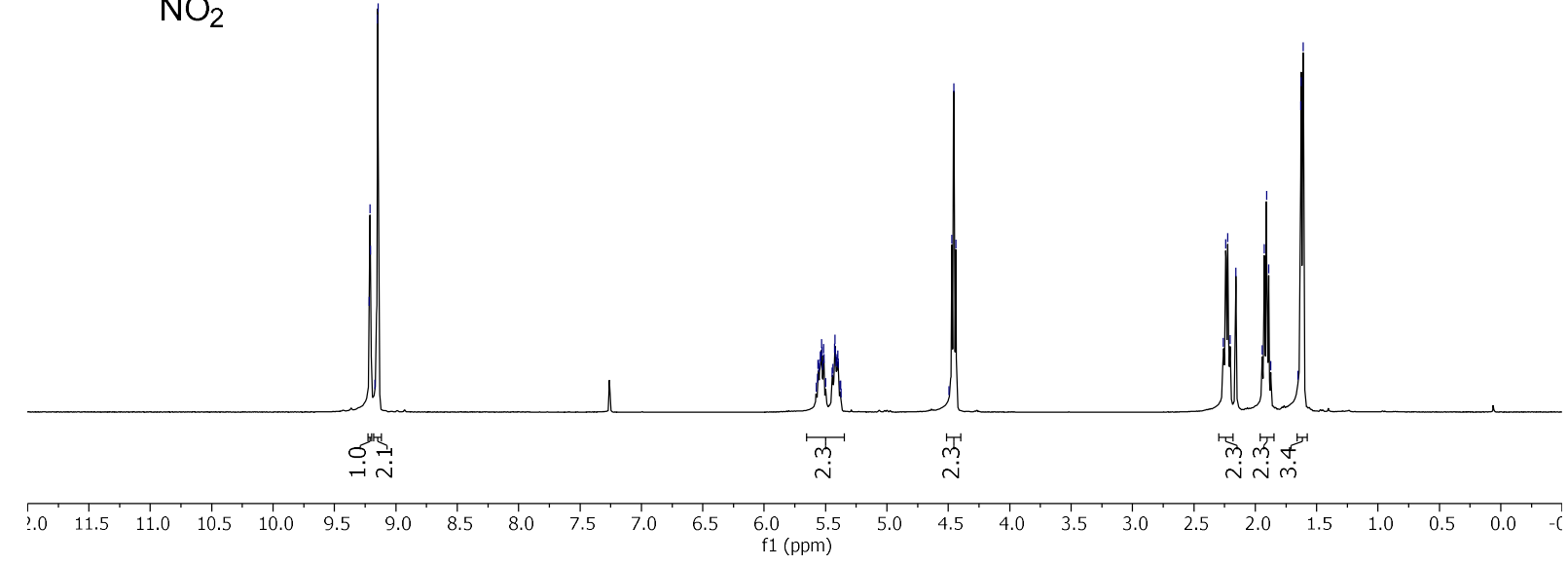

${ }^{13} \mathrm{C}$ NMR $\left(101 \mathrm{MHz}, \mathrm{CDCl}_{3}\right)$

0
1

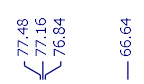

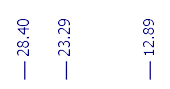
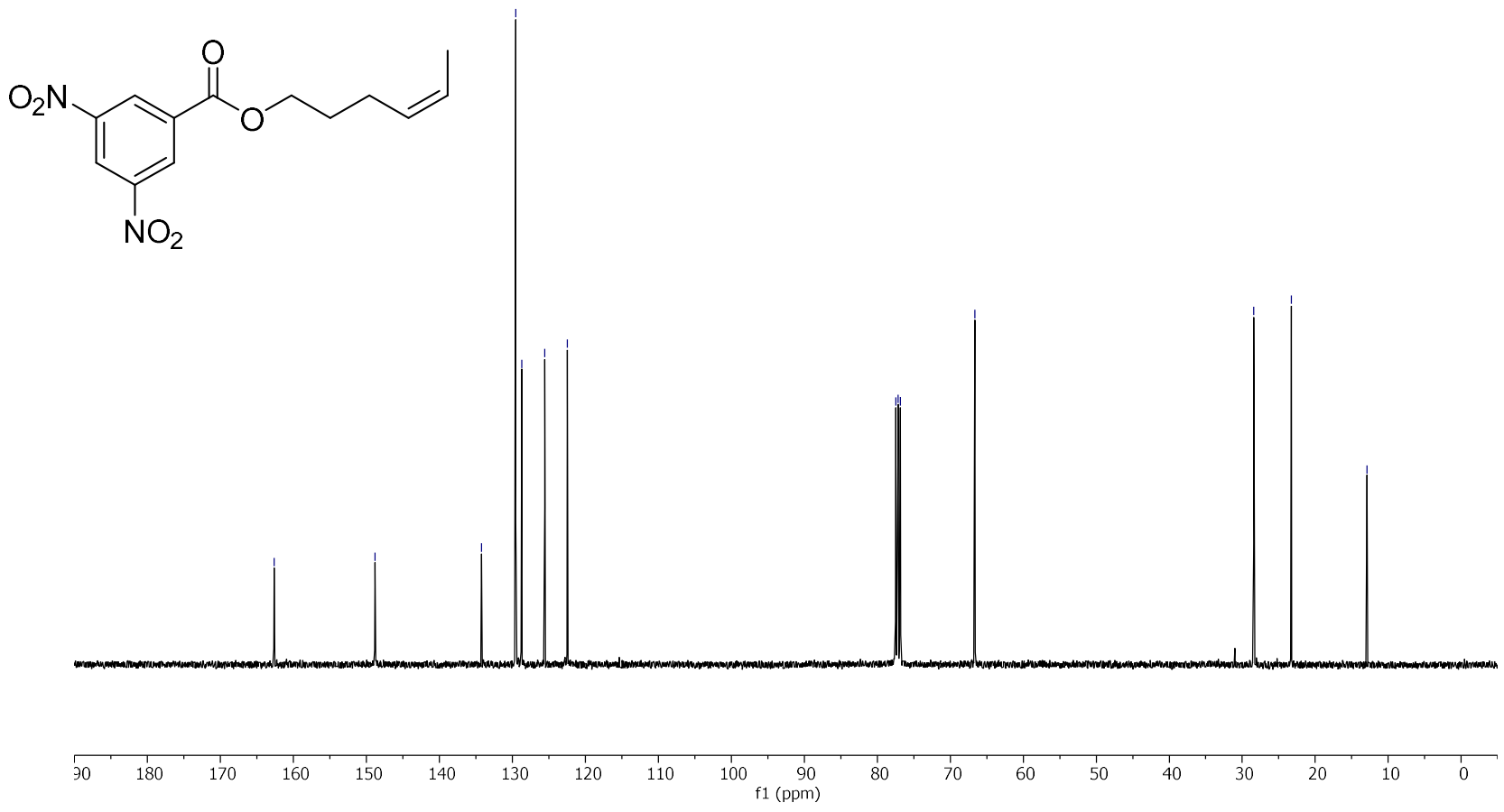

S65 
Methyl undec-10-en-1-yl sulfate (S10)

${ }^{1} \mathrm{H}$ NMR $\left(400 \mathrm{MHz}, \mathrm{CDCl}_{3}\right)$

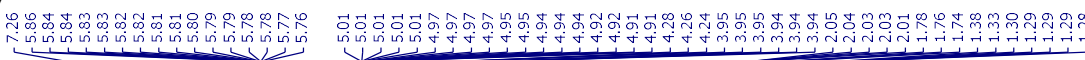

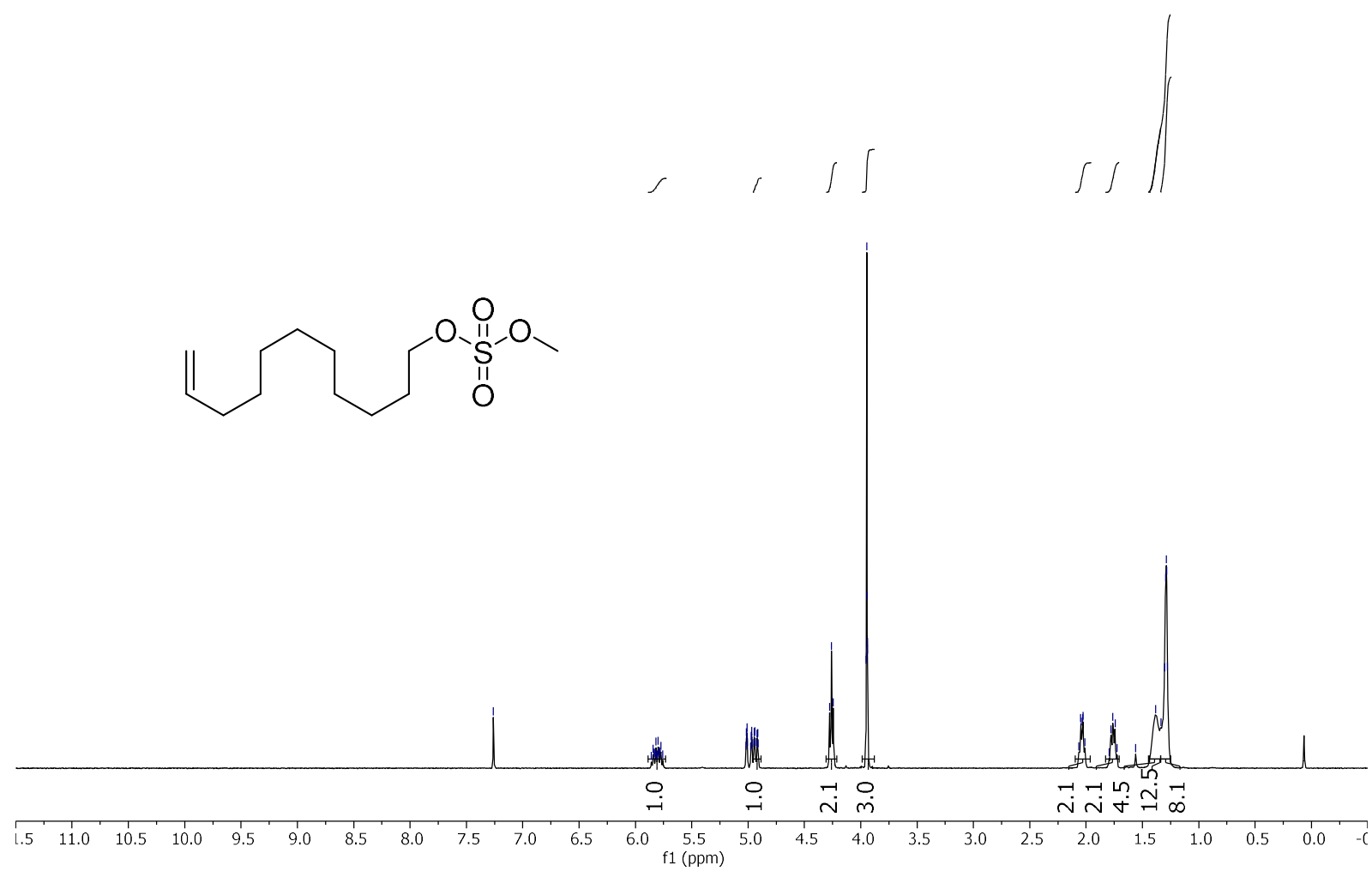

${ }^{13} \mathrm{C}$ NMR $\left(101 \mathrm{MHz}, \mathrm{CDCl}_{3}\right)$

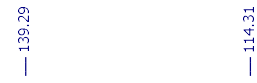

|

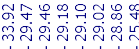<smiles>C=CCCCCCCCCOS(=O)(=O)OC</smiles>

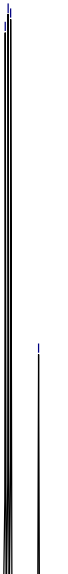

70

$160 \quad 150$

140

130

120 


\section{Diphenyl undec-10-en-1-yl phosphate (S11)}

${ }^{1} \mathrm{H} \mathrm{NMR}\left(400 \mathrm{MHz}, \mathrm{CDCl}_{3}\right)$
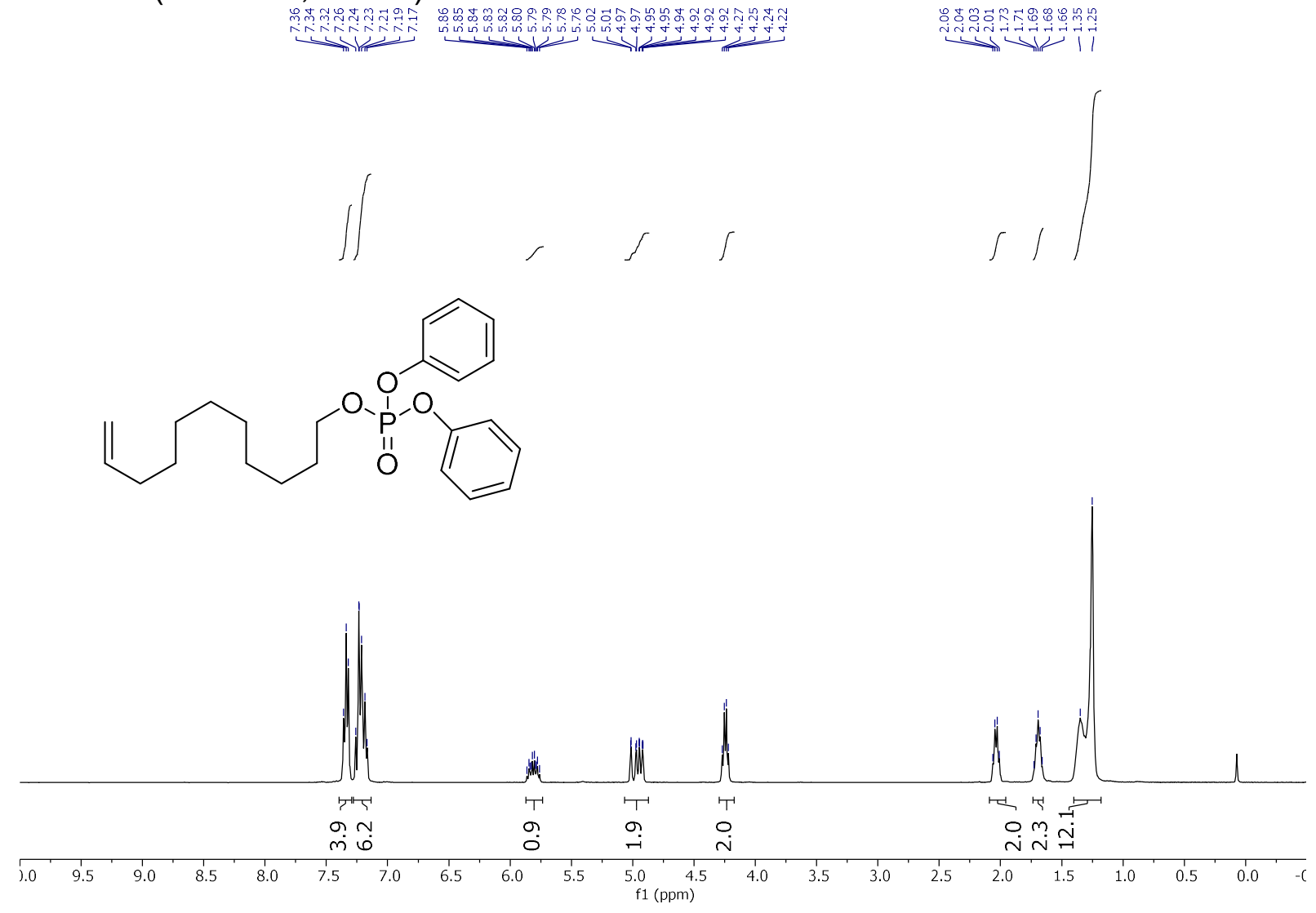

$\left.{ }^{13} \mathrm{C} \mathrm{NMR} \mathrm{(101} \mathrm{MHz,} \mathrm{CDCl}_{3}\right)$

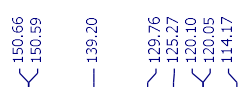<smiles>C=CCCCCCCCCCOP(=O)(Oc1ccccc1)Oc1ccccc1</smiles>
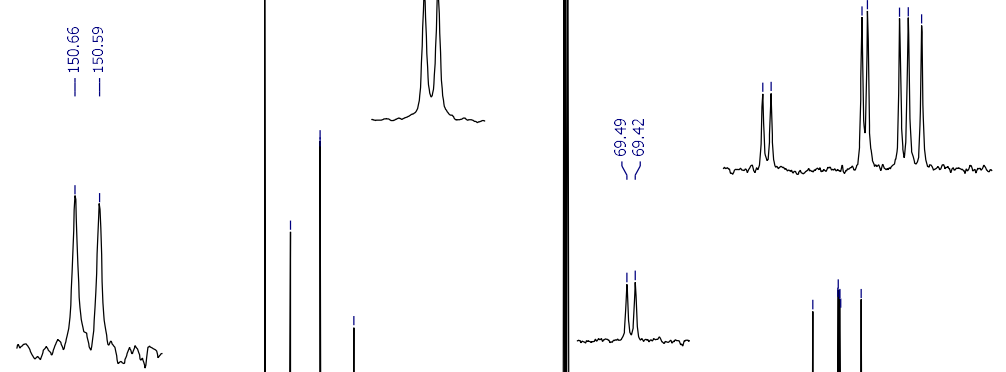
${ }^{31} \mathrm{P}$ NMR (162 MHz, $\left.\mathrm{CDCl}_{3}\right)$
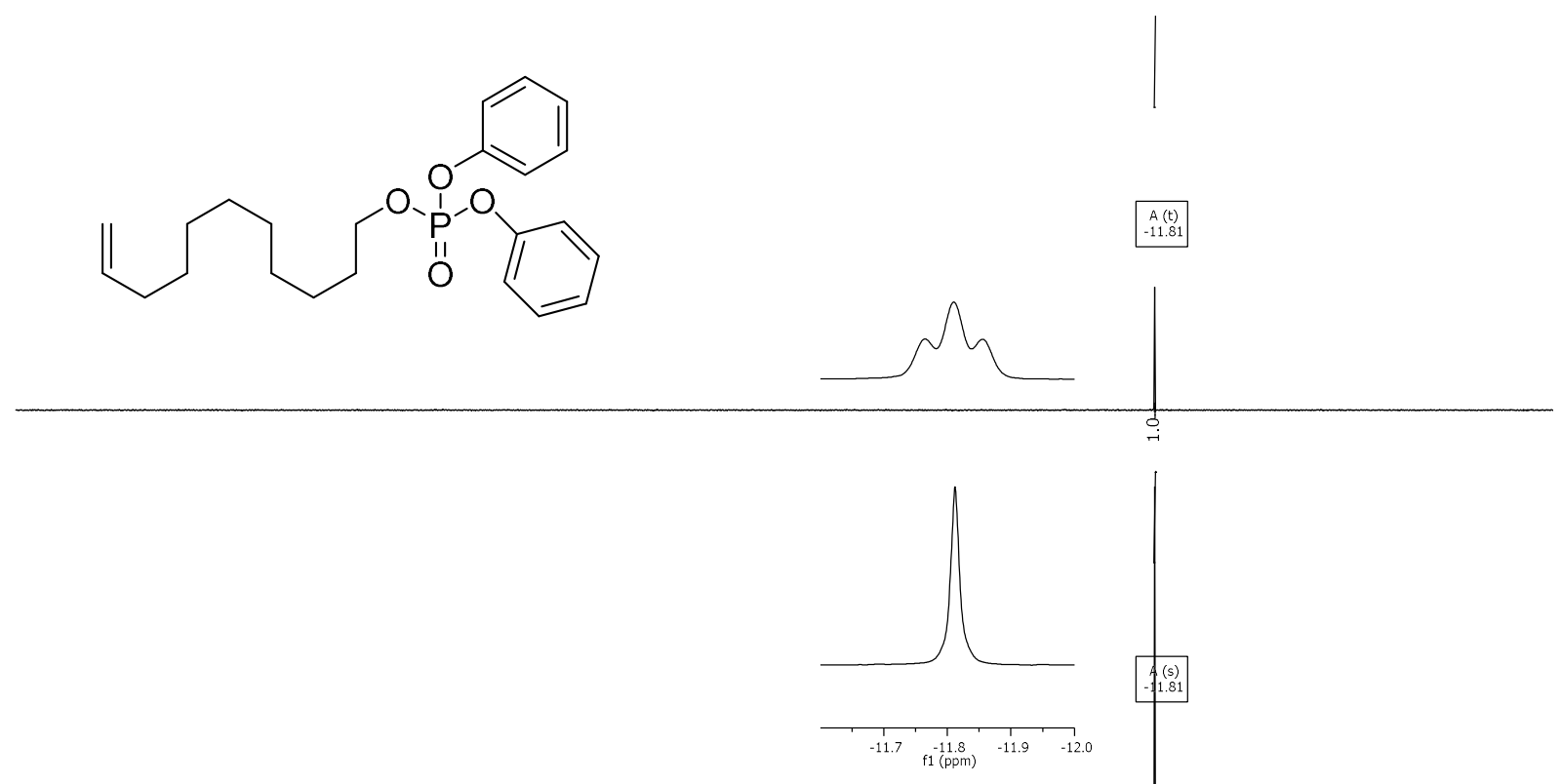

Dichloro(p-tolyl)- $\boldsymbol{\lambda}^{\mathbf{3}}$-iodane (S15)

${ }^{1} \mathrm{H}$ NMR (400 MHz, CDCl 3 )<smiles>Cc1ccc(I(Cl)Cl)cc1</smiles>
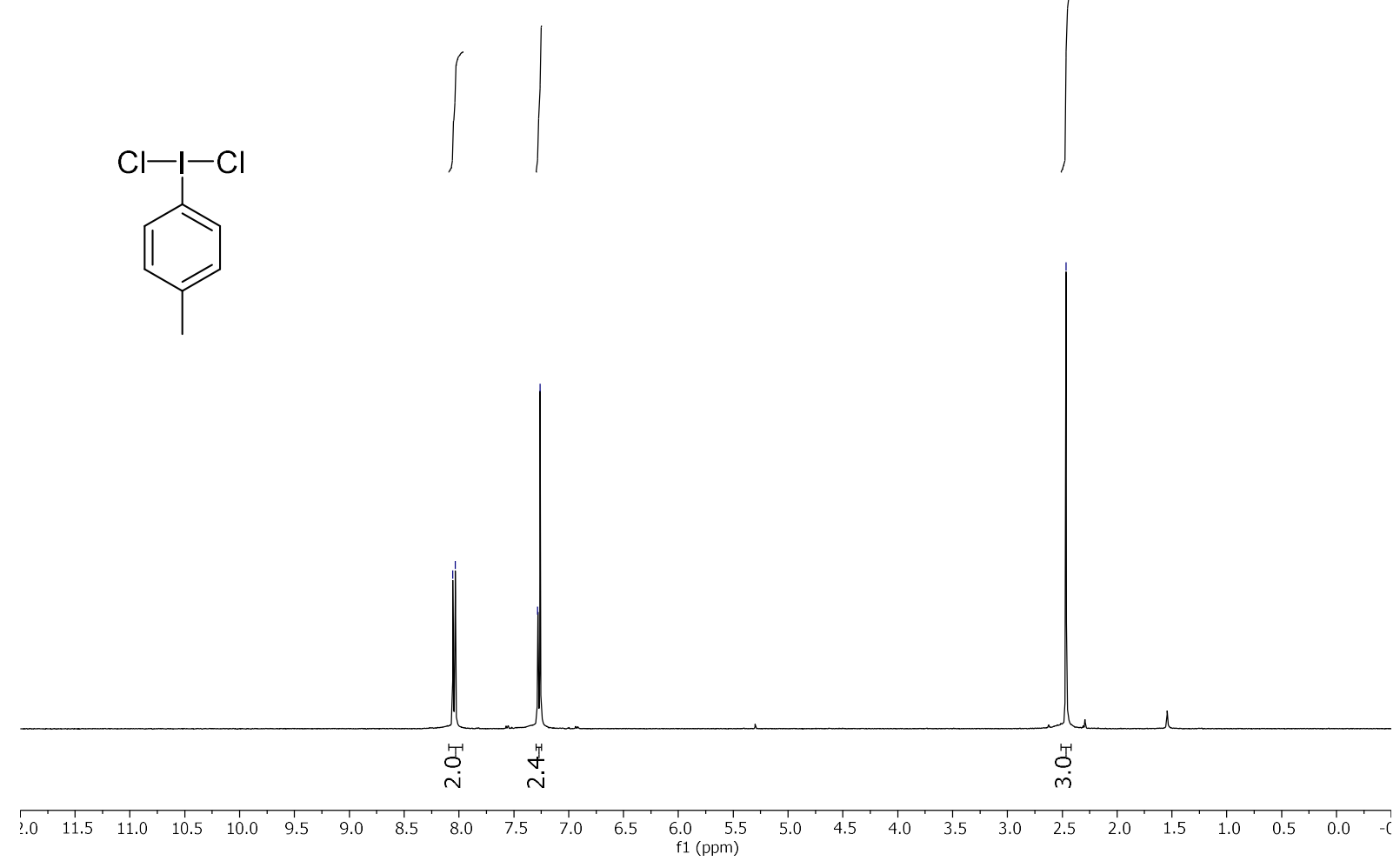
XI. NMR Spectra of products

5,6-Dichlorohexyl benzoate (2)

${ }^{1} \mathrm{H}$ NMR $\left(500 \mathrm{MHz}, \mathrm{CDCl}_{3}\right)$

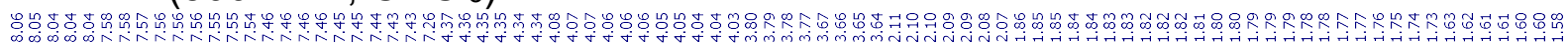
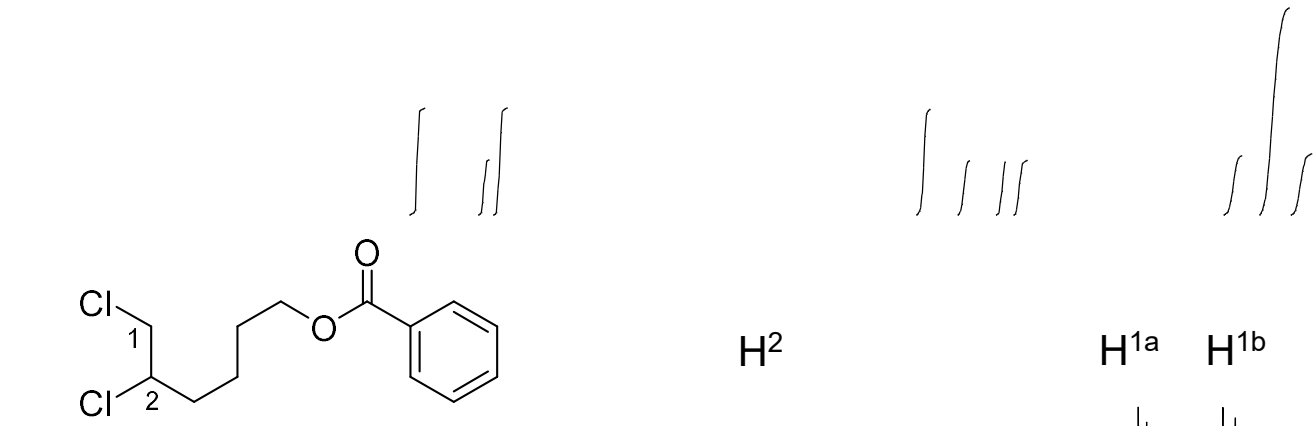

$\mathrm{H}^{2}$

$H^{1 a} \quad H^{1 b}$

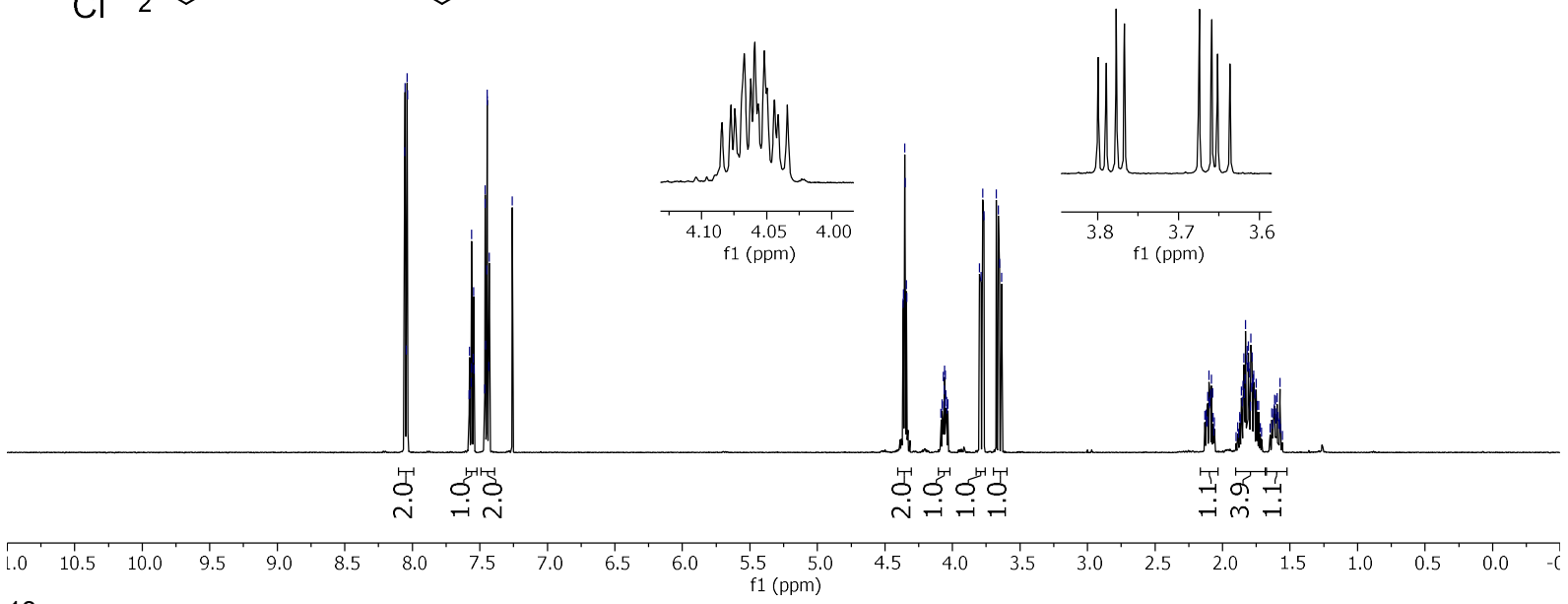

${ }^{13} \mathrm{C}$ NMR (126 MHz, $\left.\mathrm{CDCl}_{3}\right)$

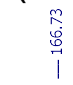

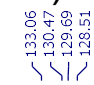

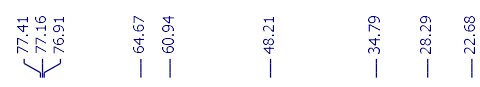
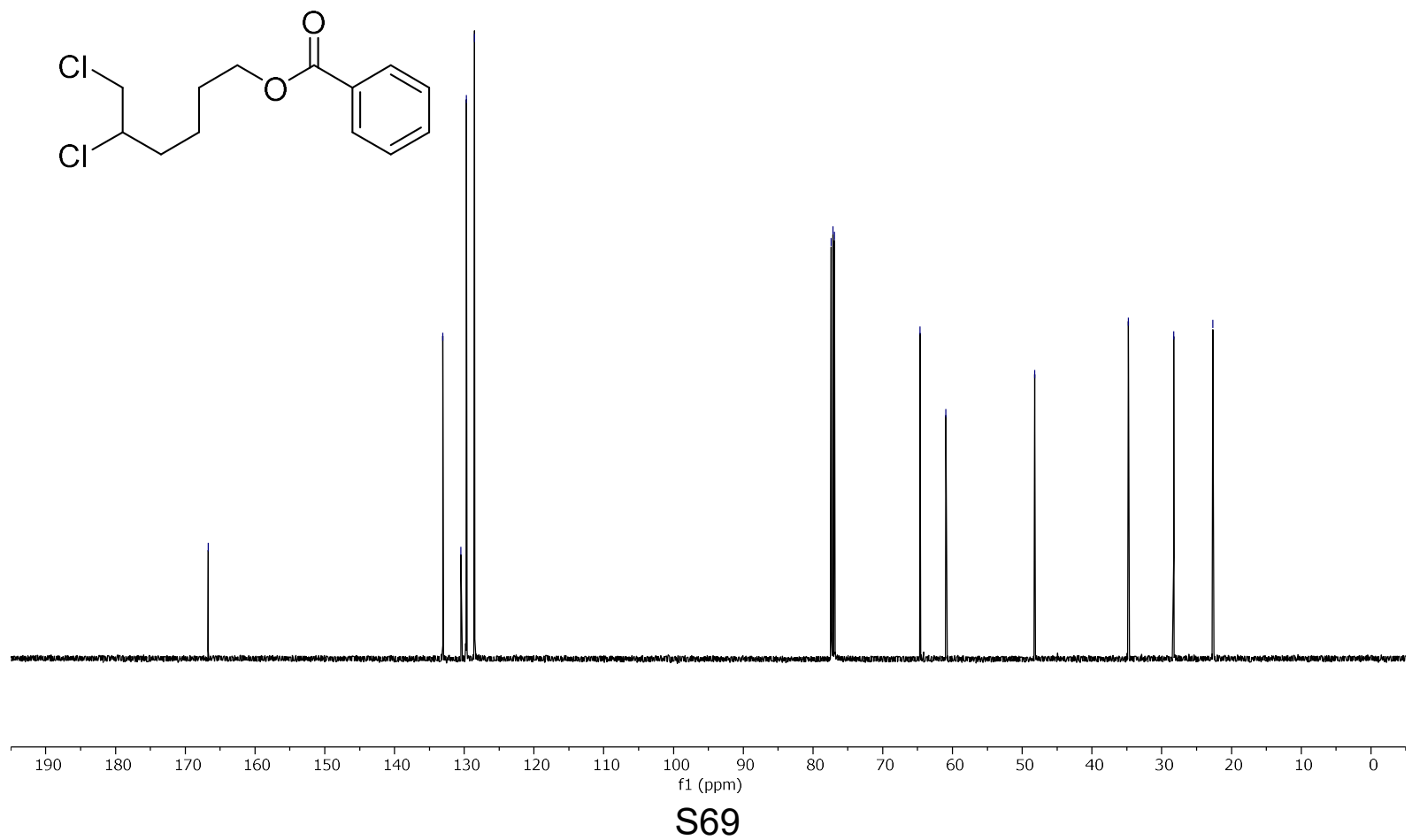


\section{0,11-Dichloroundecyl acetate (3)}

${ }^{1} \mathrm{H} \mathrm{NMR}\left(400 \mathrm{MHz}, \mathrm{CDCl}_{3}\right)$

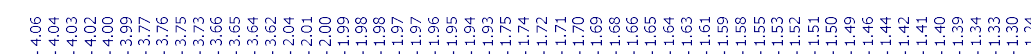<smiles>CC(=O)OCCCCCCCCCC(Cl)CCl</smiles>

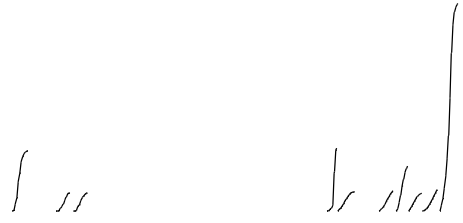

$H^{1 a} \quad H^{1 b}$

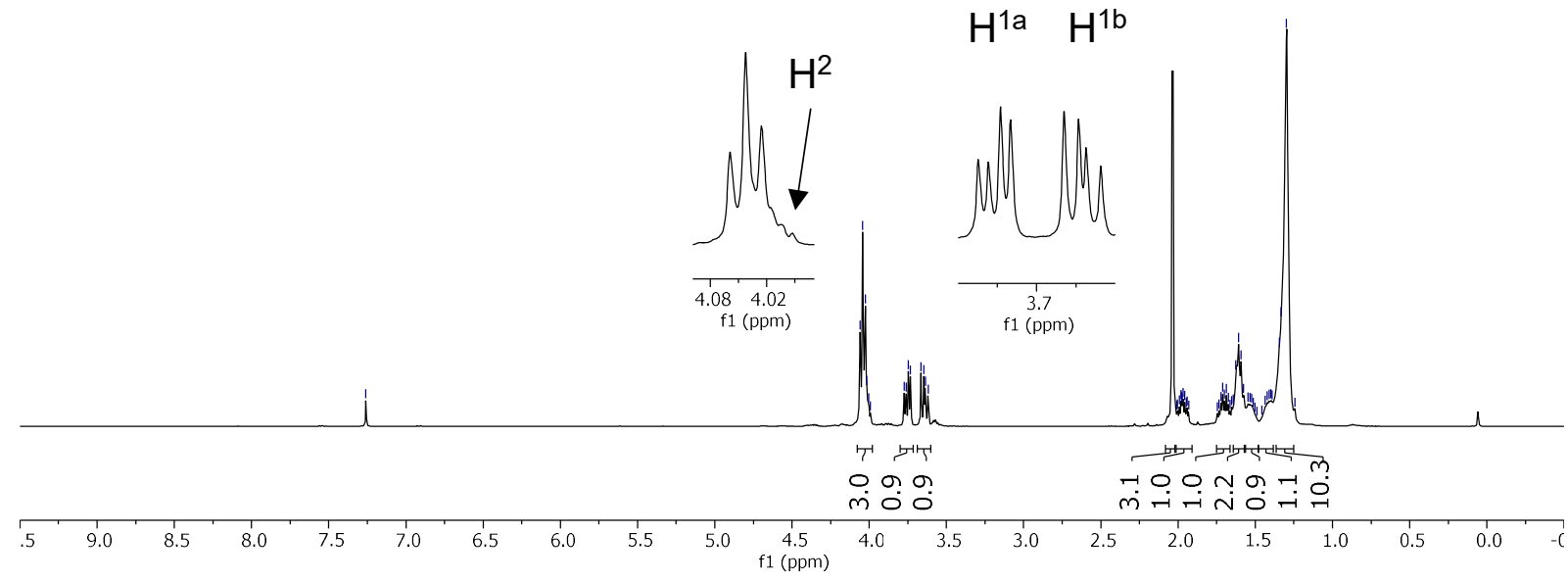

${ }^{13} \mathrm{C}$ NMR $\left(101 \mathrm{MHz}, \mathrm{CDCl}_{3}\right)$<smiles>CC(=O)OCCCCCCCCCC(Cl)CCl</smiles>

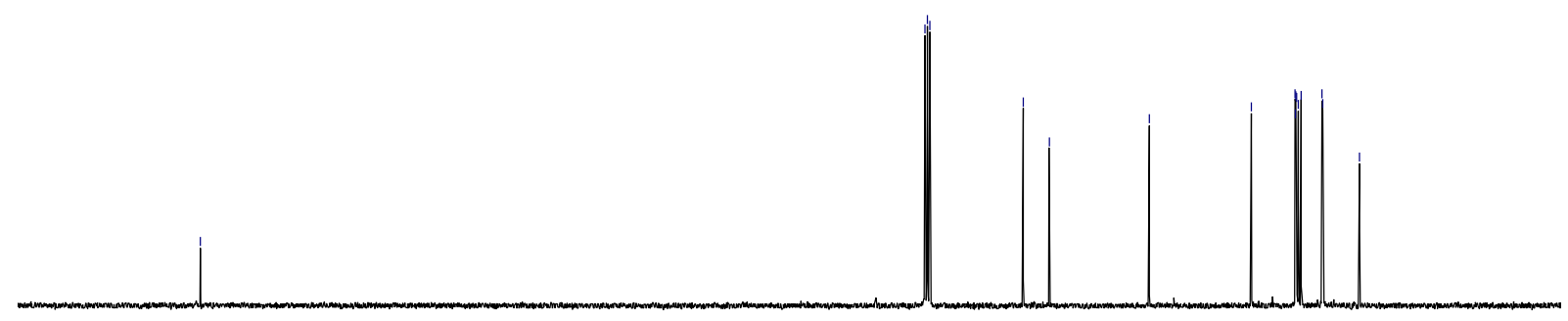

$\begin{array}{llllllllllllllllllllll}1 & 190 & 180 & 170 & 160 & 150 & 140 & 130 & 120 & 110 & \underset{100}{10} f_{1(\mathrm{ppm})} & 80 & 70 & 60 & 50 & 40 & 30 & 20 & 10 & 0\end{array}$ 


\section{0,11-Dichloroundecyl methacrylate (4)}

${ }^{1} \mathrm{H}$ NMR $\left(400 \mathrm{MHz}, \mathrm{CDCl}_{3}\right)$

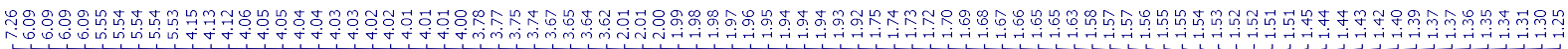

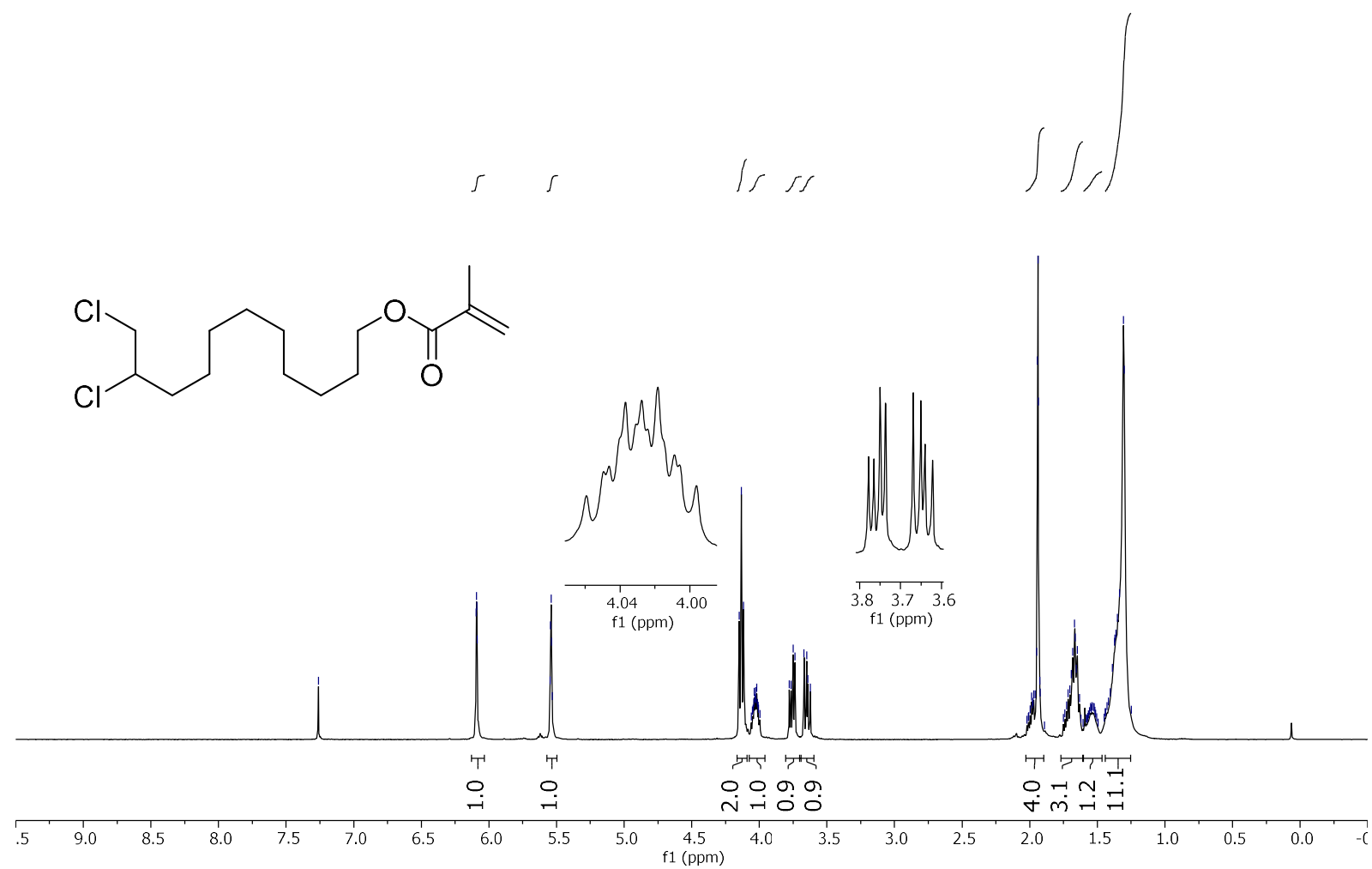

${ }^{13} \mathrm{C}$ NMR $\left(101 \mathrm{MHz}, \mathrm{CDCl}_{3}\right)$<smiles>C=C(C)C(=O)OCCCCCCCCC(Cl)CCl</smiles>
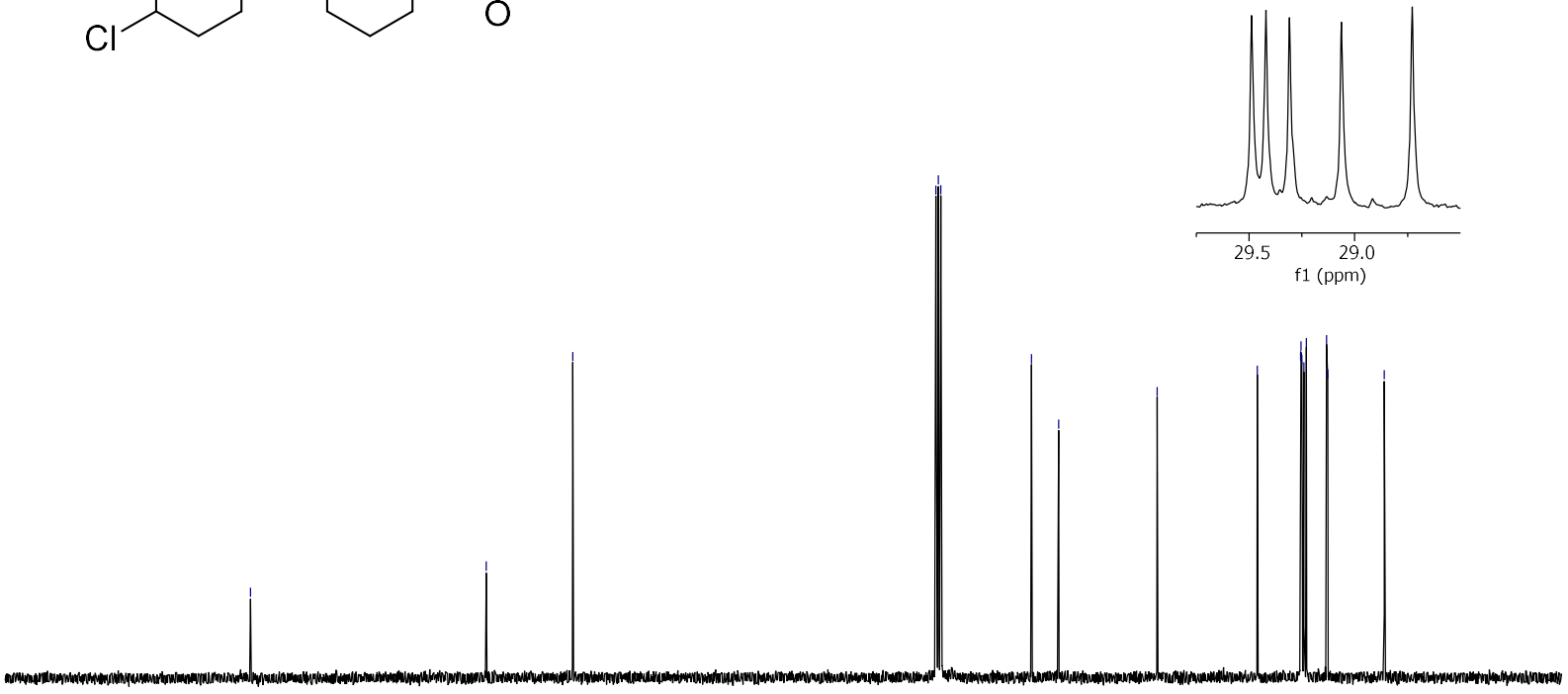

\begin{tabular}{llllllllllllllllllllll}
\hline 0 & 190 & 180 & 170 & 160 & 150 & 140 & 130 & 120 & 110 & 100 & 90 & 80 & 70 & 60 & 50 & 40 & 30 & 20 & 10 & 0
\end{tabular} 
10,11-Dichloroundecan-1-ol (5)

${ }^{1} \mathrm{H}$ NMR $\left(400 \mathrm{MHz}, \mathrm{CDCl}_{3}\right)$

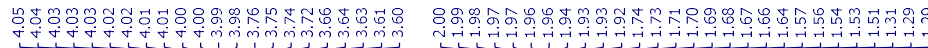

1,1

, 11

$\int \mathrm{Cl}^{\mathrm{OH}}$
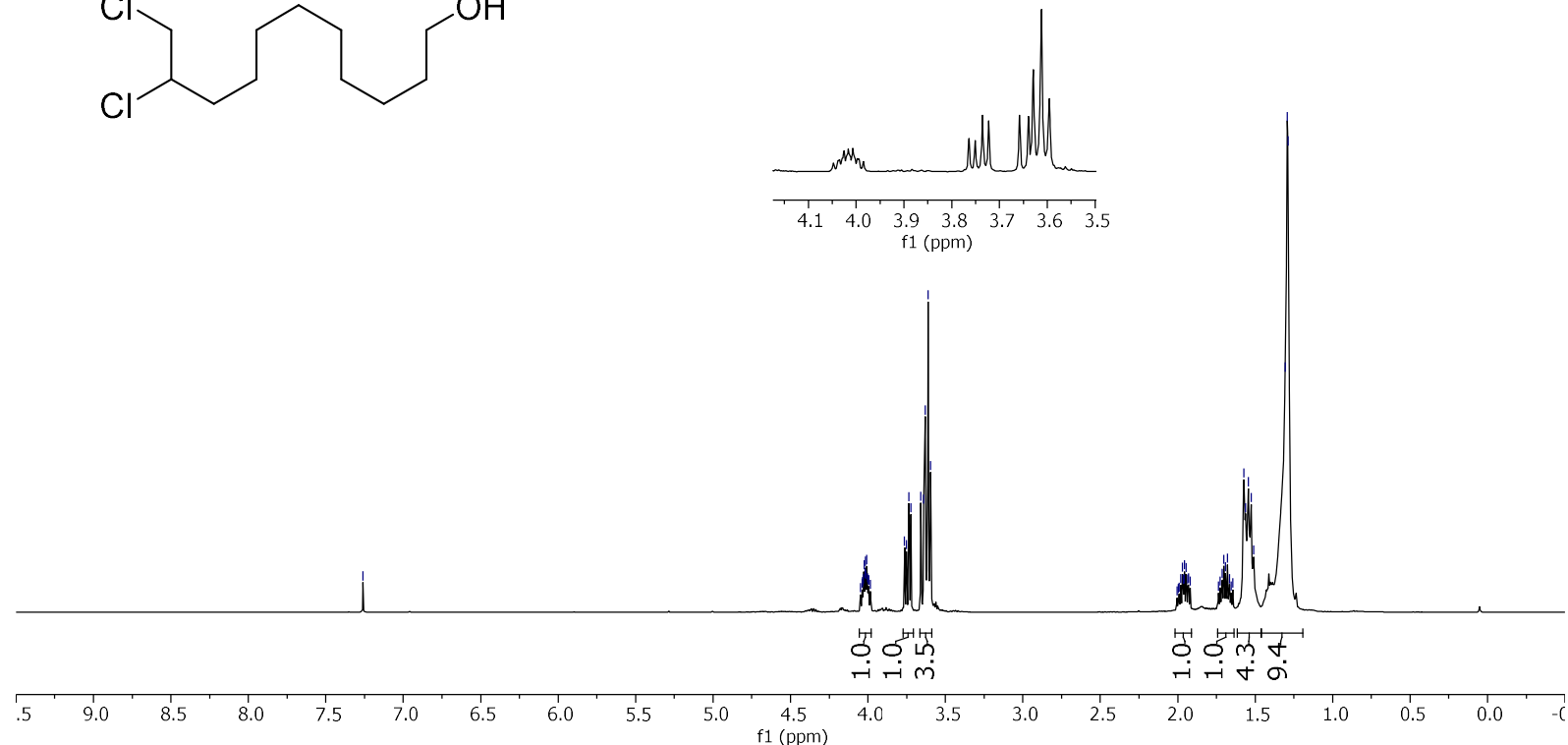

10,11-Dichloroundecanoic acid (6)

${ }^{1} \mathrm{H} \mathrm{NMR}\left(300 \mathrm{MHz}, \mathrm{CDCl}_{3}\right)$

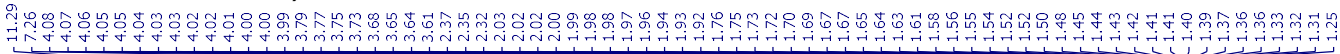<smiles>O=C(O)CCCCCCCC(Cl)CCl</smiles>

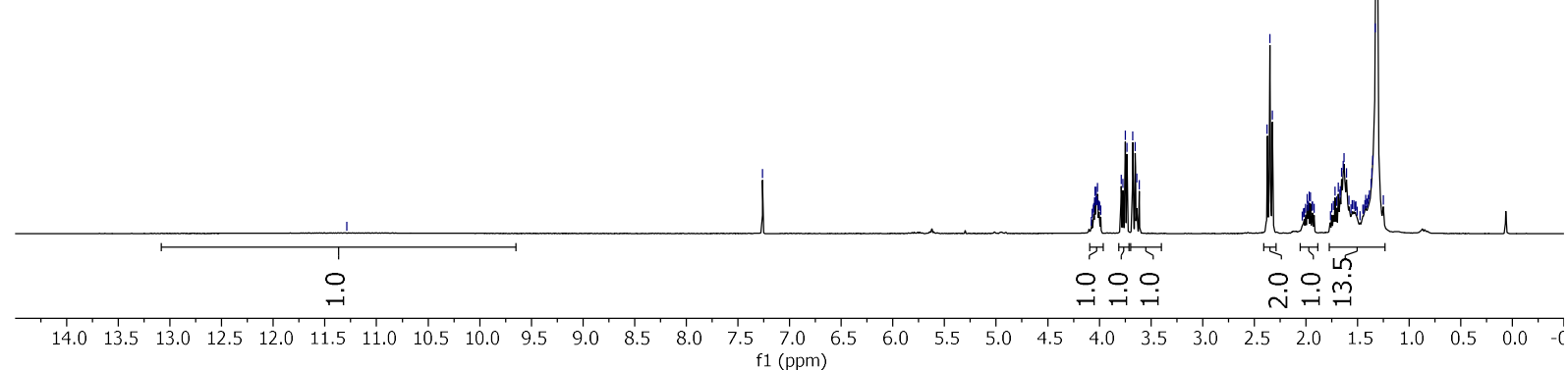




\section{1-Bromo-1,2-dichloroundecane (7)}

${ }^{1} \mathrm{H} \mathrm{NMR}\left(400 \mathrm{MHz}, \mathrm{CDCl}_{3}\right.$

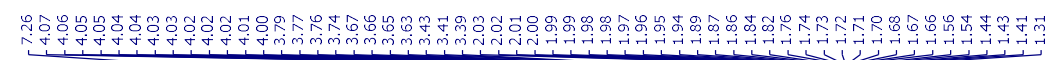
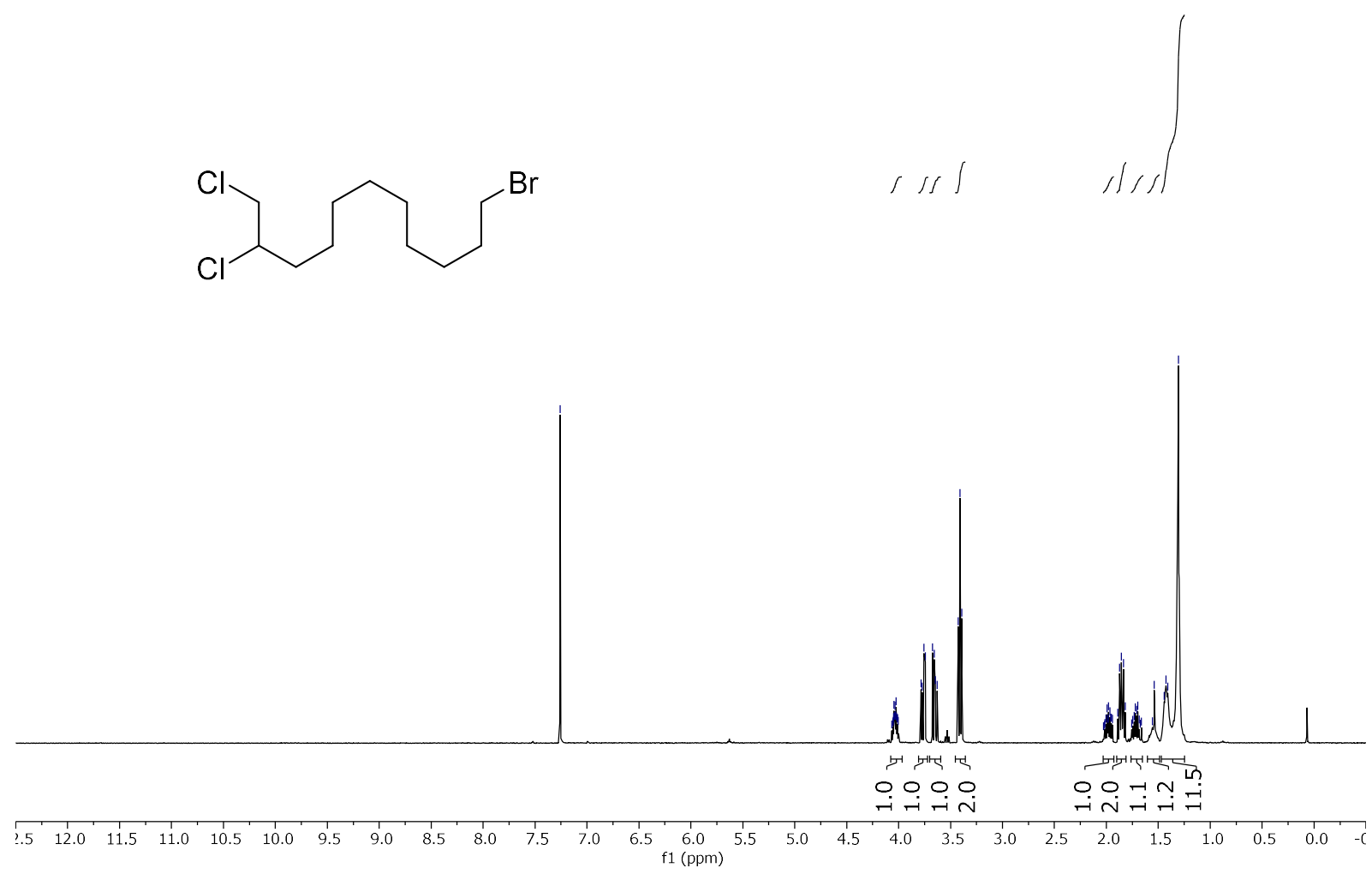
10,11-Dichloroundecyl methyl sulfate (8)

${ }^{1} \mathrm{H}$ NMR $\left(599 \mathrm{MHz}, \mathrm{CDCl}_{3}\right)$

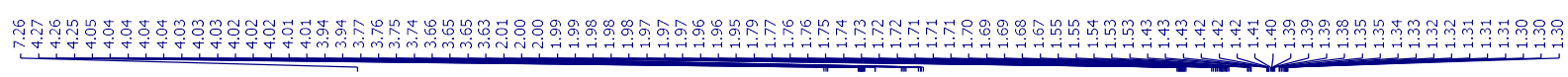

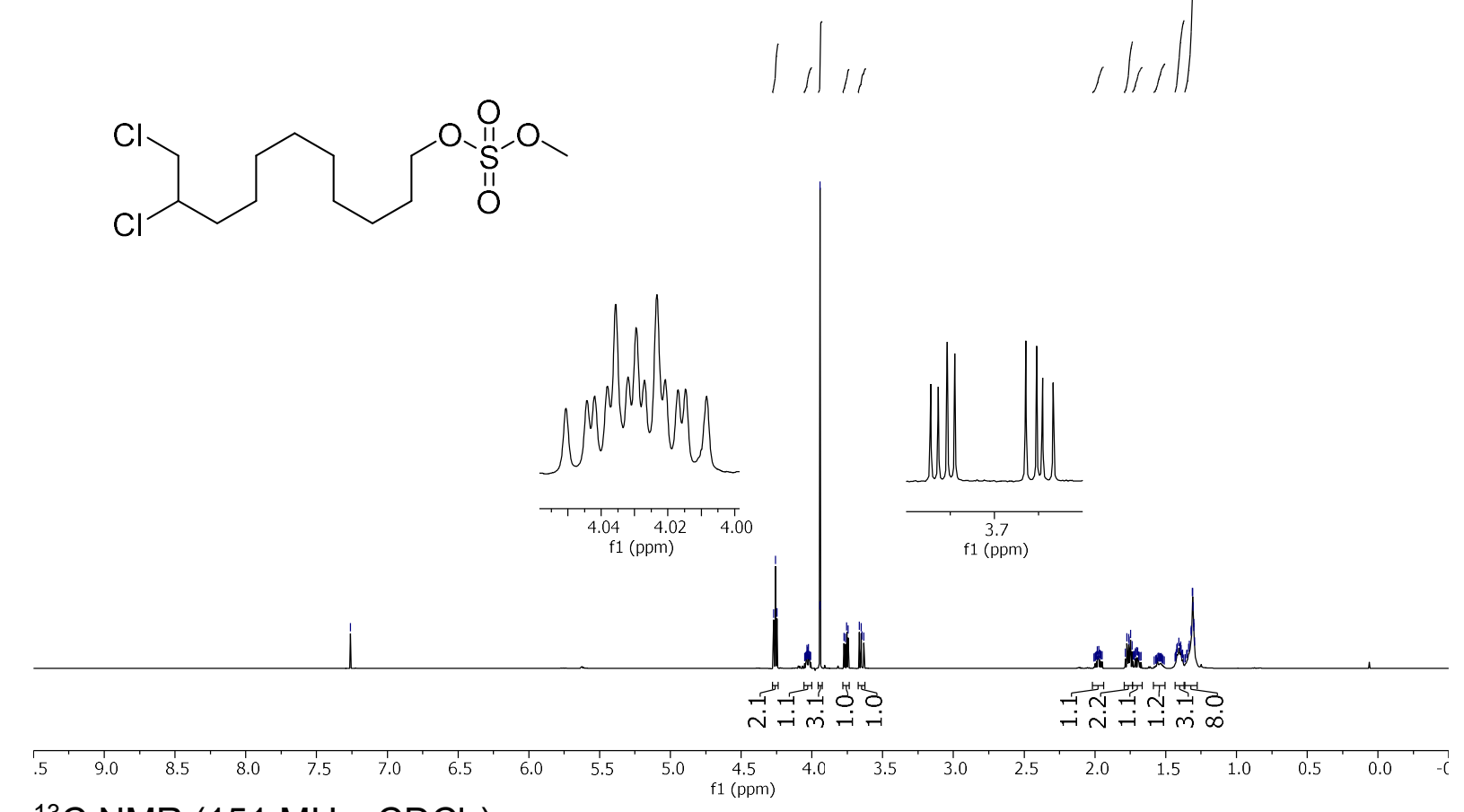

${ }^{13} \mathrm{C} \mathrm{NMR}\left(151 \mathrm{MHz}, \mathrm{CDCl}_{3}\right)$<smiles>COS(=O)(=O)OCCCCCCCCCC(Cl)CCl</smiles>

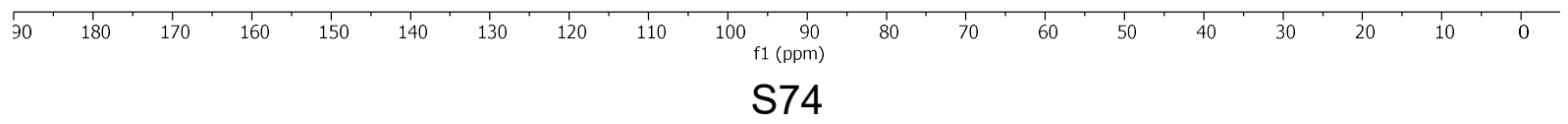




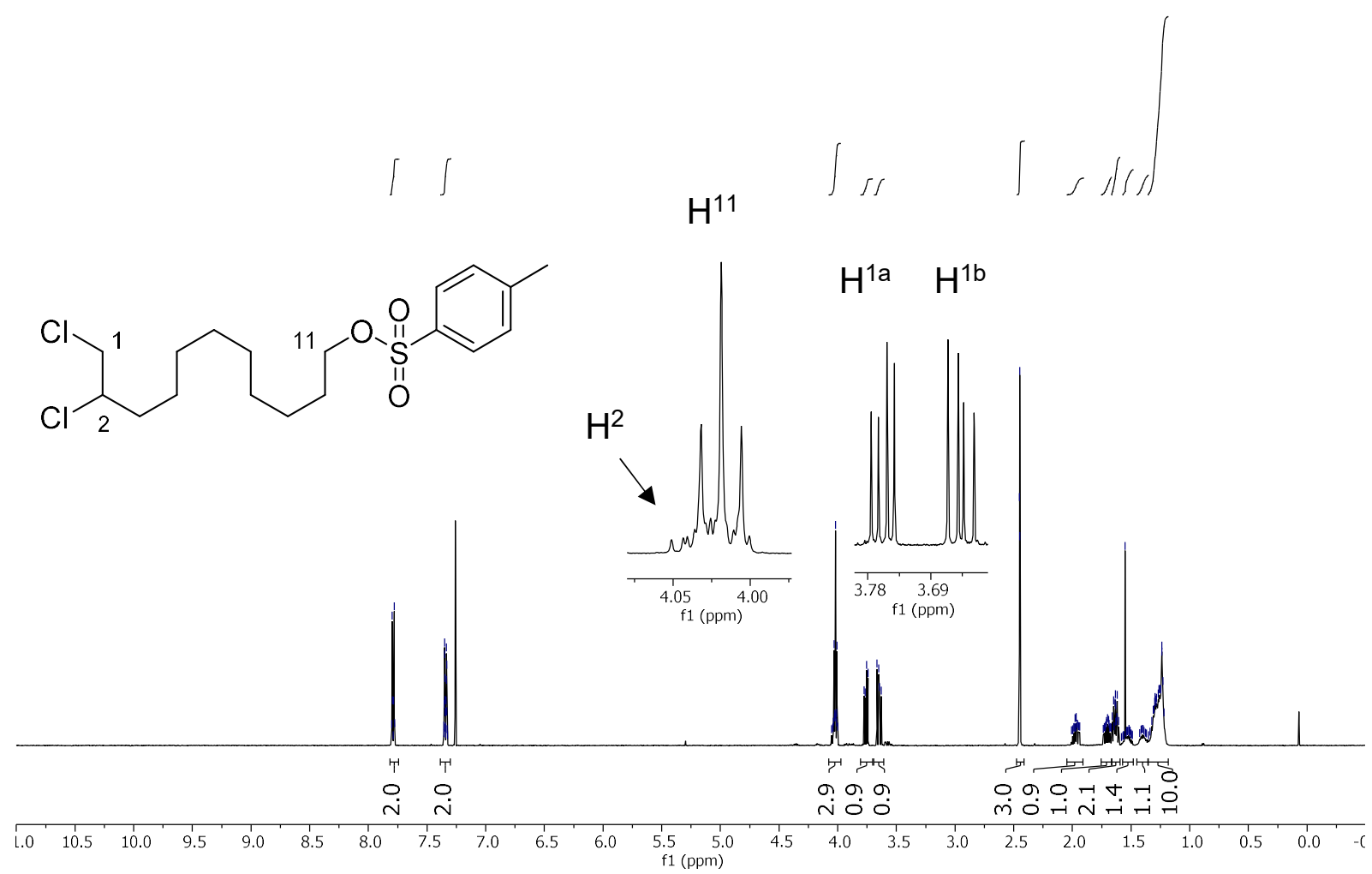

$\left.{ }^{13} \mathrm{C} \mathrm{NMR} \mathrm{(126} \mathrm{MHz,} \mathrm{CDCl}_{3}\right)$<smiles>Cc1ccc(S(=O)(=O)OCCCCCCCCCC(Cl)CCl)cc1</smiles>

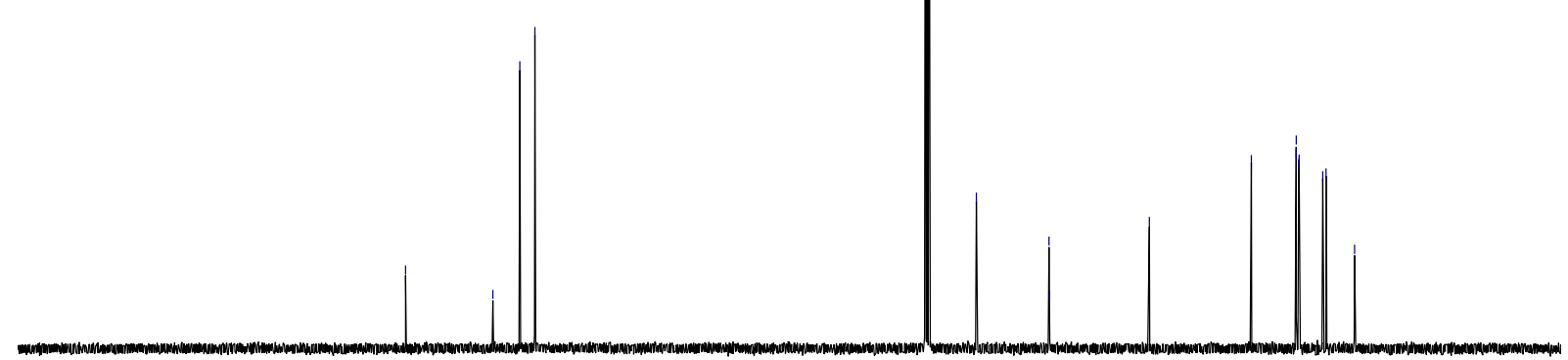




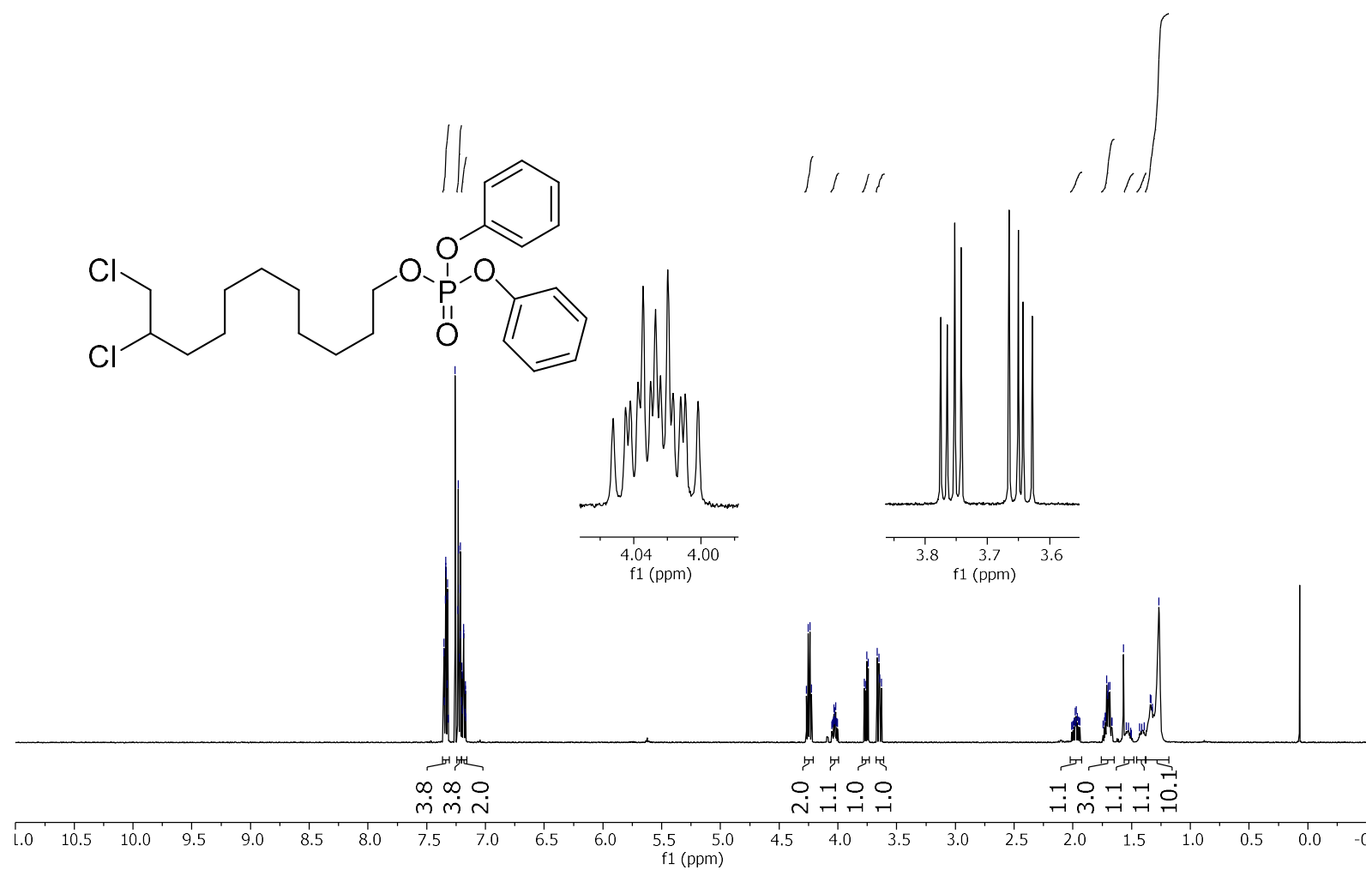

${ }^{13} \mathrm{C} \mathrm{NMR}\left(101 \mathrm{MHz}, \mathrm{CDCl}_{3}\right)$

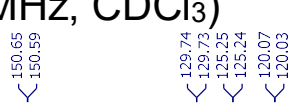

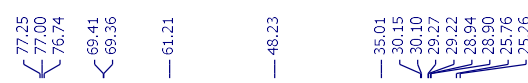<smiles>O=P(OCCCCCCCCC(Cl)CCl)(Oc1ccccc1)Oc1ccccc1</smiles>

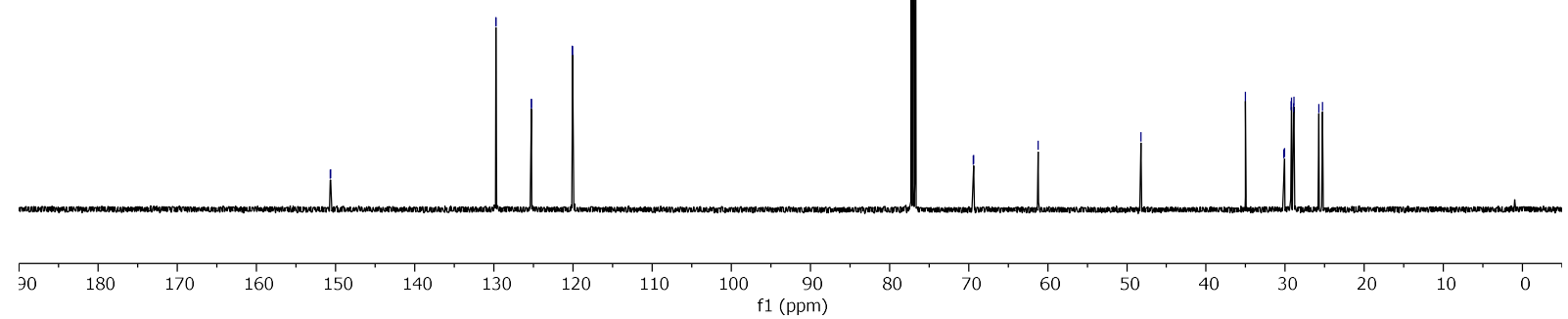


${ }^{31} \mathrm{P}$ NMR $\left(202 \mathrm{MHz}, \mathrm{CDCl}_{3}\right)$

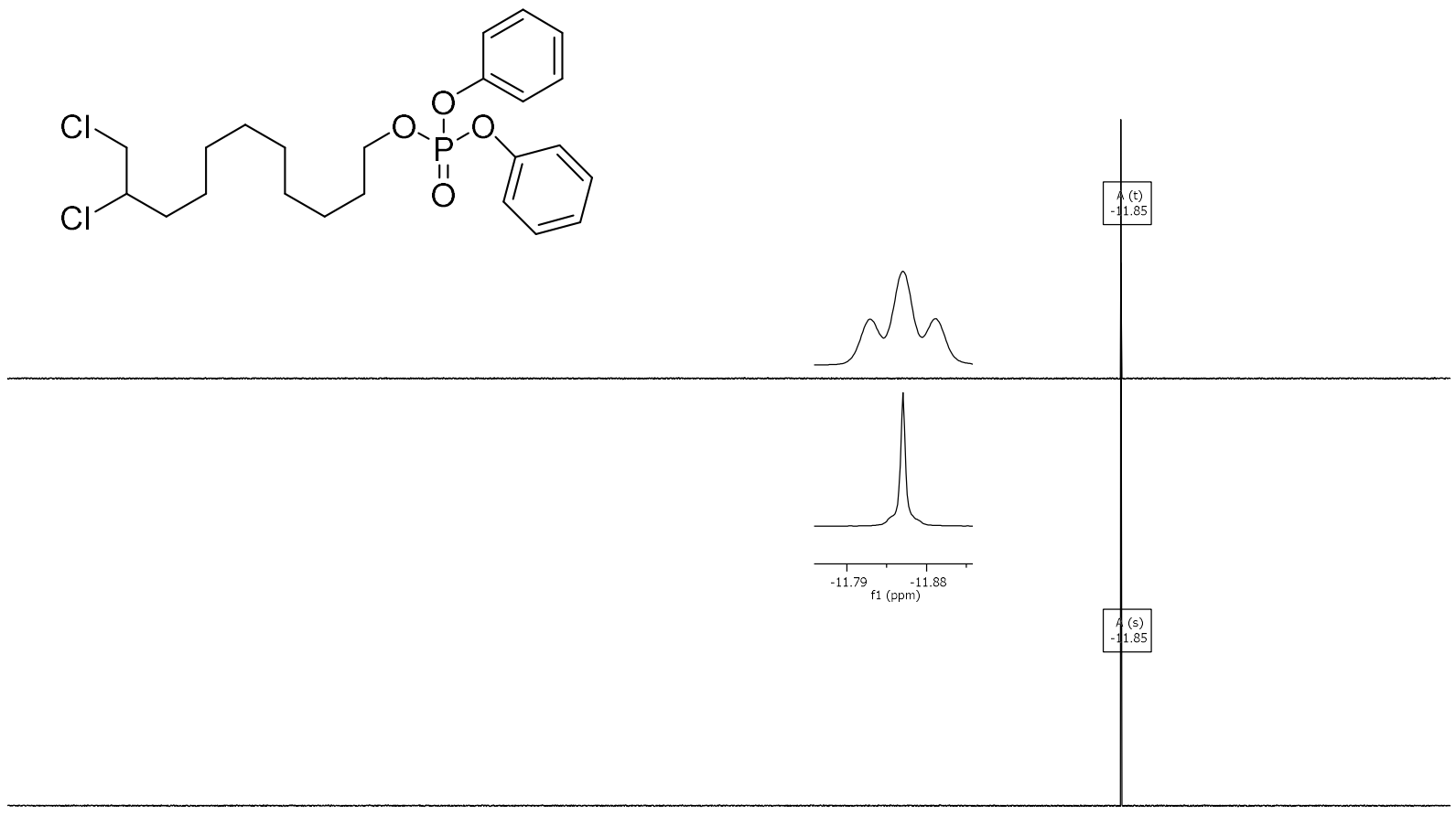

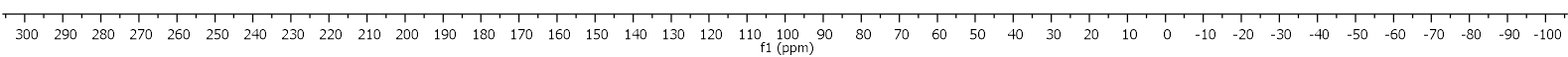




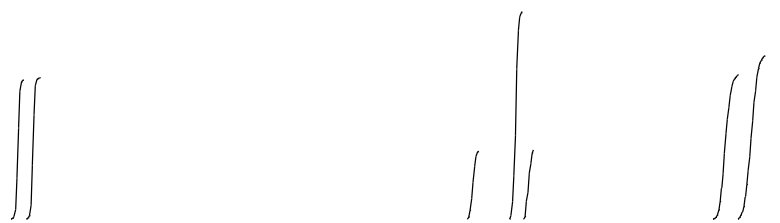<smiles>O=C1c2ccccc2C(=O)N1CCCC(Cl)CCl</smiles>

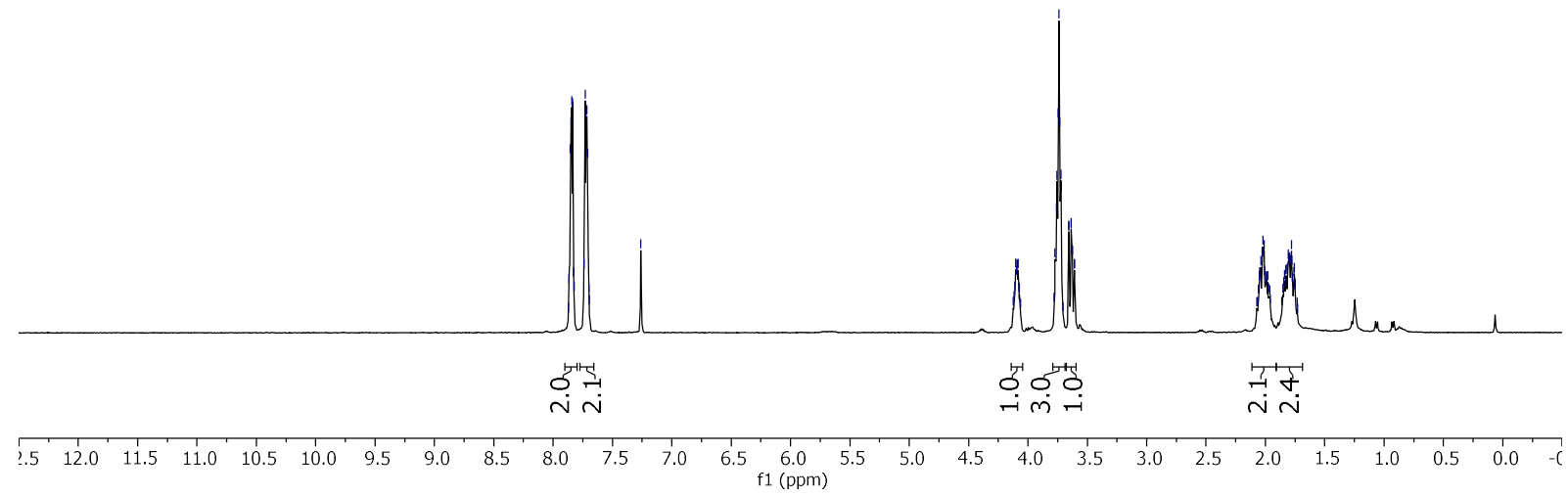

${ }^{13} \mathrm{C}$ NMR $\left(101 \mathrm{MHz}, \mathrm{CDCl}_{3}\right)$

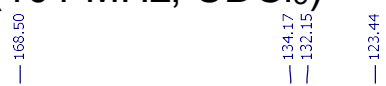<smiles>O=C1c2ccccc2C(=O)N1CCCC(Cl)CCl</smiles>
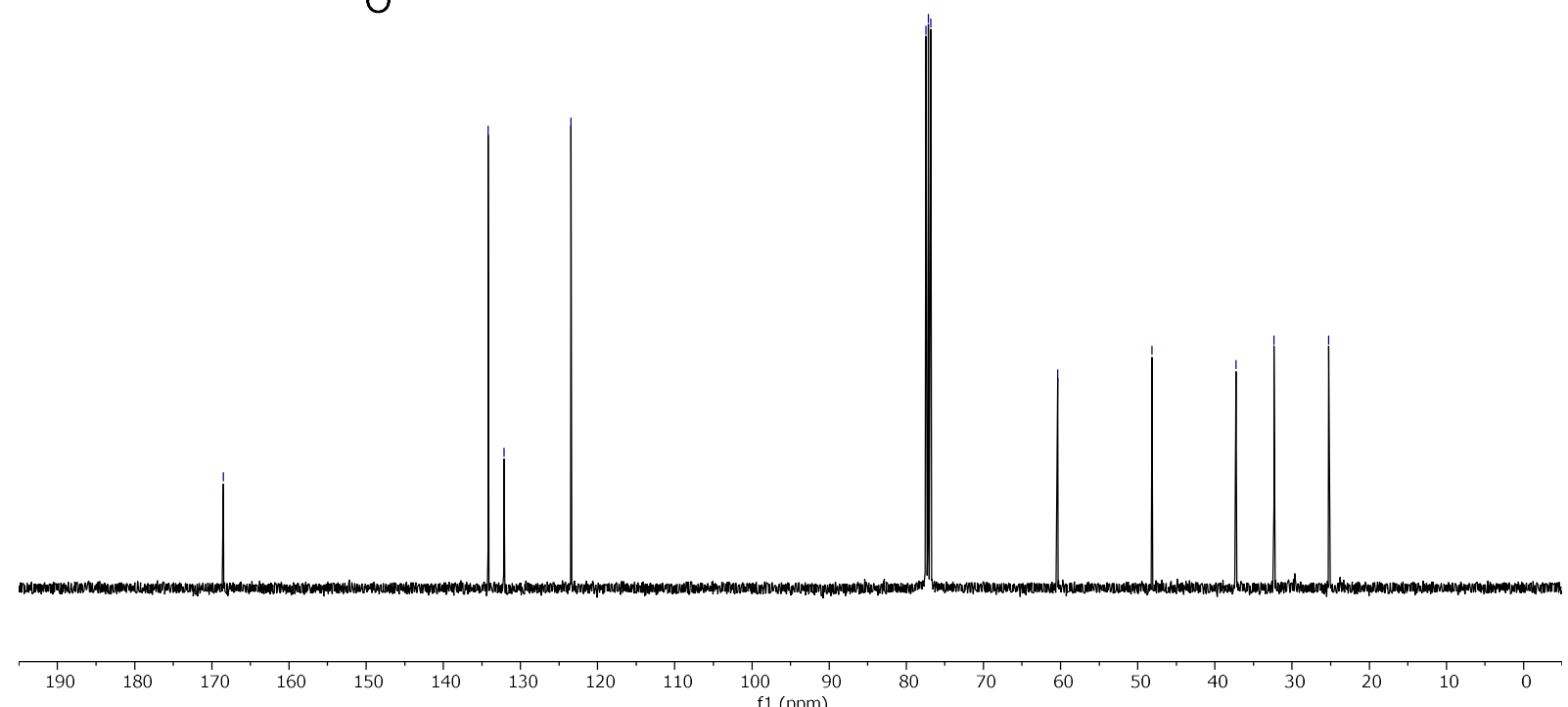
1-(2,3-Dichloropropoxy)-4-nitrobenzene (12)

${ }^{1} \mathrm{H} \mathrm{NMR}\left(400 \mathrm{MHz}, \mathrm{CDCl}_{3}\right)$

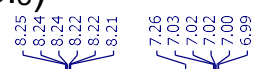

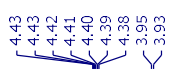<smiles>O=[N+]([O-])c1ccc(OCC(Cl)CCl)cc1</smiles>
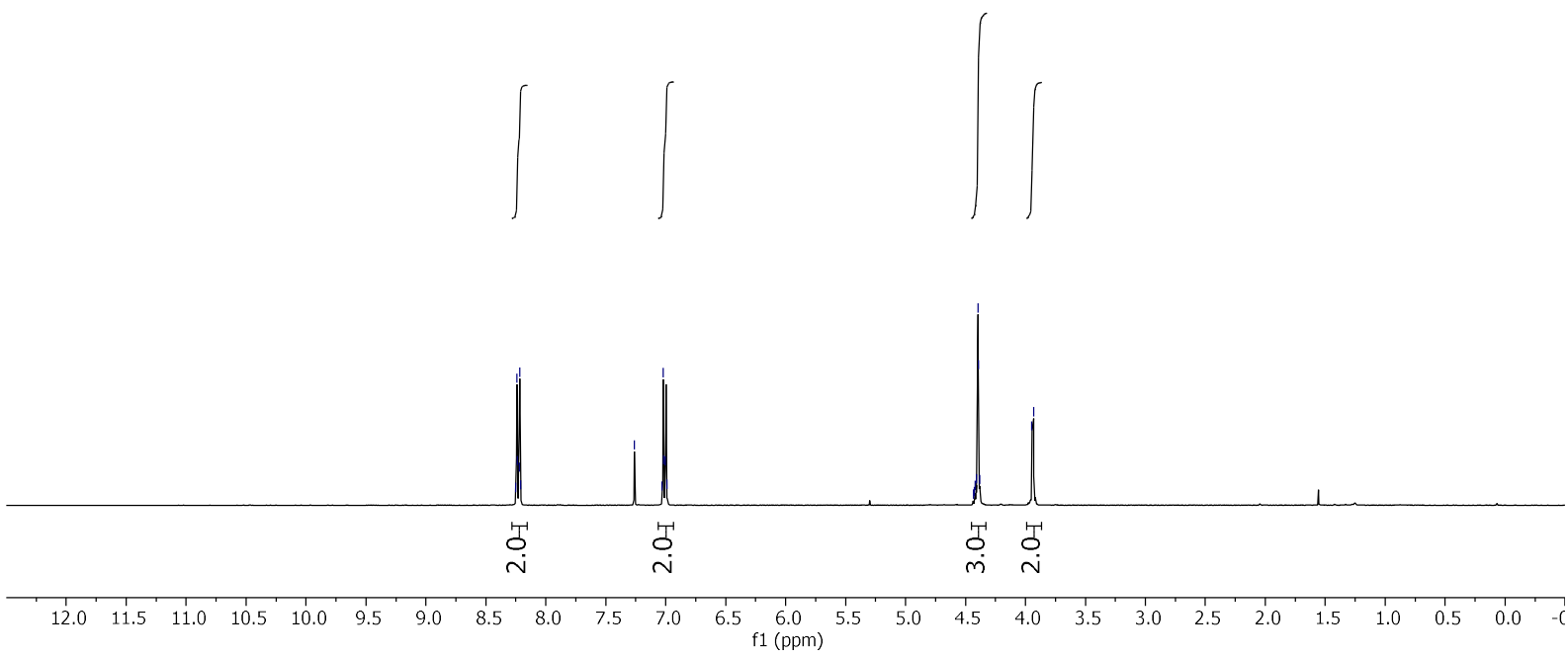

${ }^{13} \mathrm{C}$ NMR $\left(101 \mathrm{MHz}, \mathrm{CDCl}_{3}\right)$

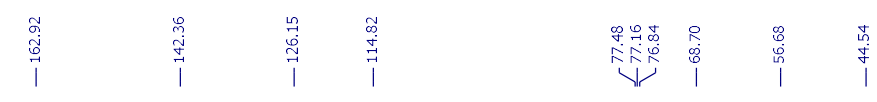<smiles>O=[N+]([O-])c1ccc(OCC(Cl)CCl)cc1</smiles>
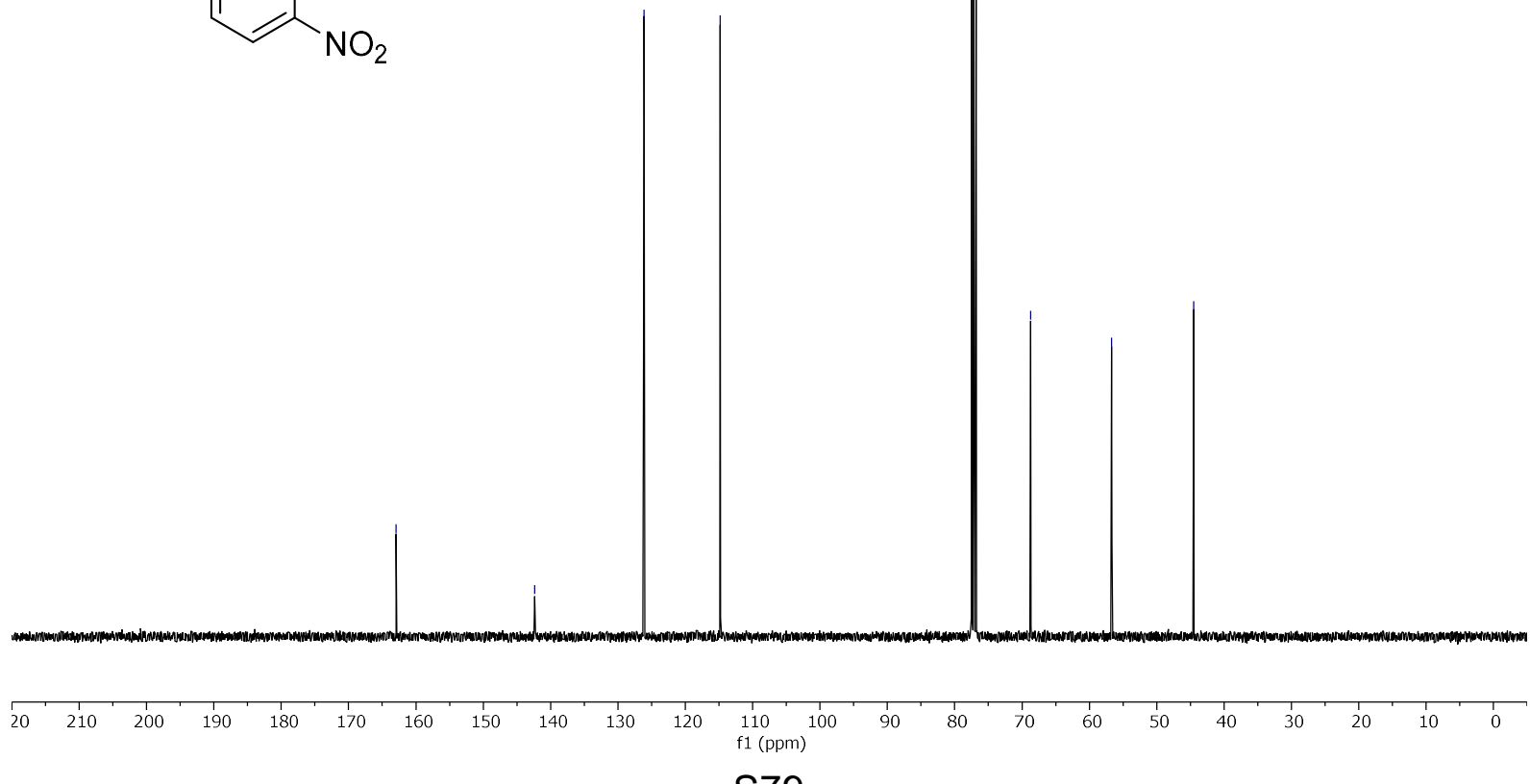


\section{2,3-Dichloropropyl 4-methylbenzenesulfonate (13)}

${ }^{1} \mathrm{H} \mathrm{NMR}\left(400 \mathrm{MHz}, \mathrm{CDCl}_{3}\right)$

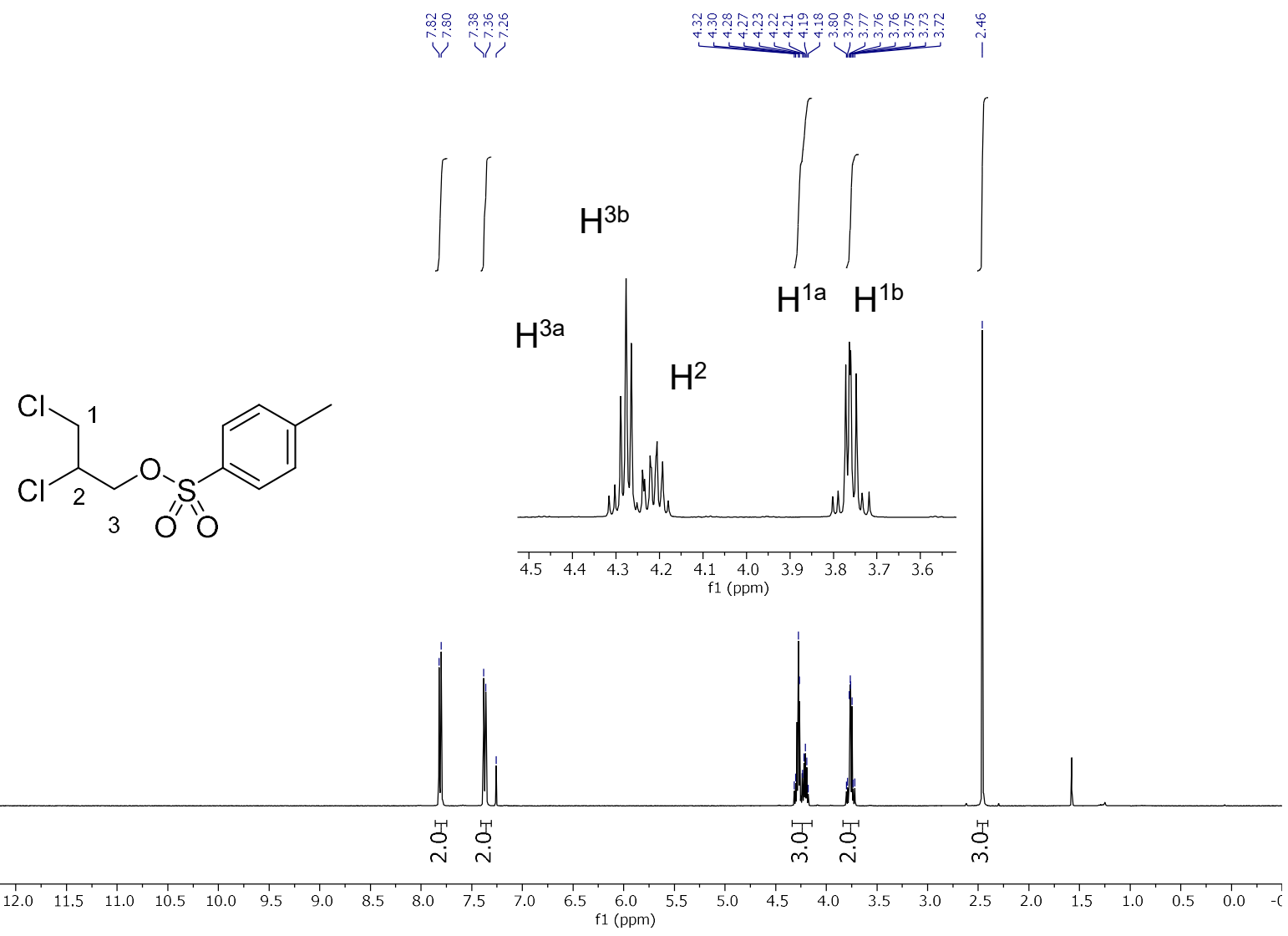

${ }^{13} \mathrm{C}$ NMR $\left(101 \mathrm{MHz}, \mathrm{CDCl}_{3}\right)$

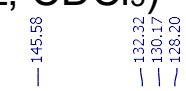<smiles>Cc1ccc(S(=O)(=O)OCC(Cl)CCl)cc1</smiles>

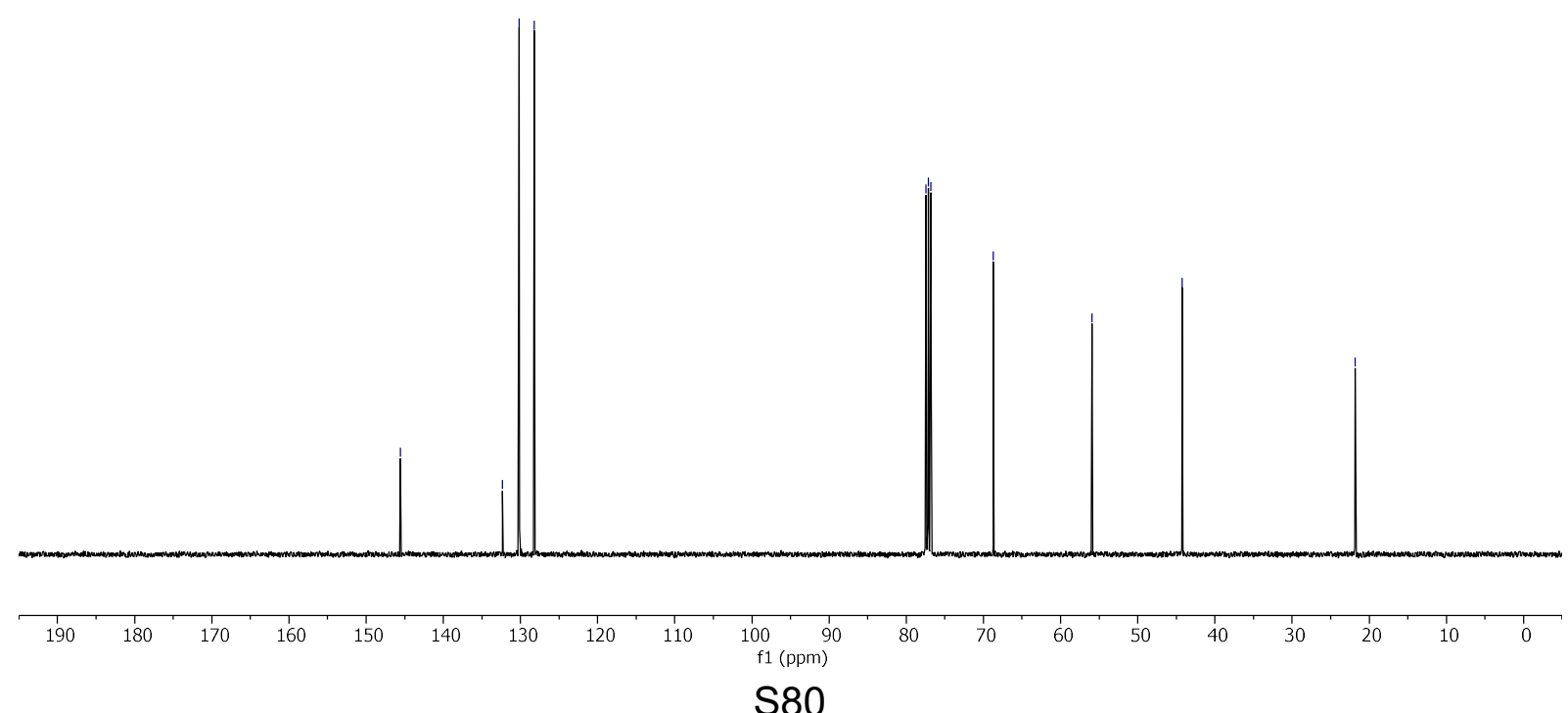


1-Chloro-4-(1,2-dichloroethyl)benzene (14)

${ }^{1} \mathrm{H} \mathrm{NMR}\left(300 \mathrm{MHz} \mathrm{CDCl}_{3}\right)$

$$
\text { J }
$$

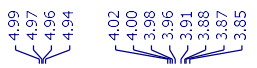

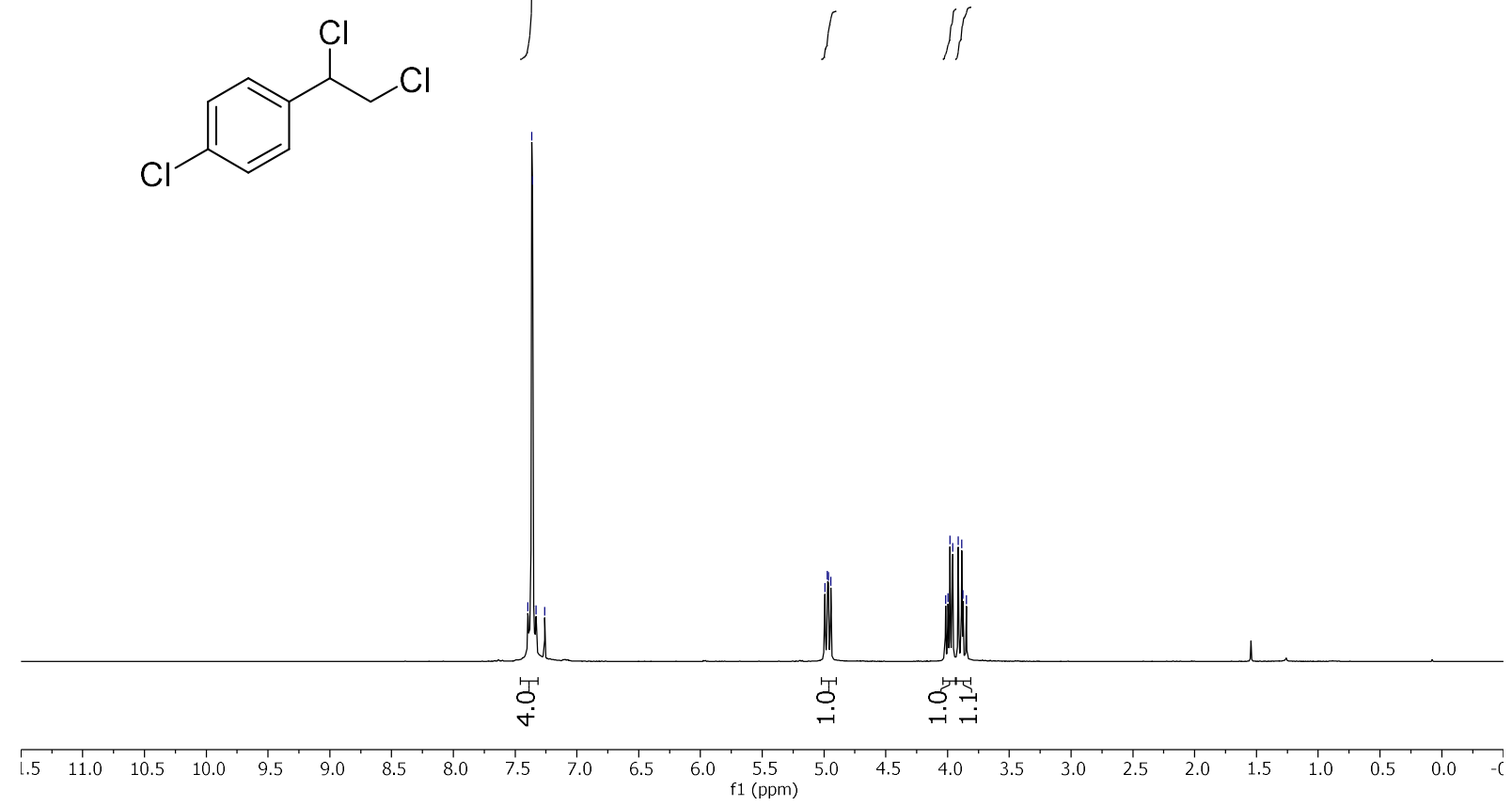

1-Bromo-4-(1,2-dichloroethyl)benzene (15)

${ }^{1} \mathrm{H}$ NMR $\left(300 \mathrm{MHz} \mathrm{CDCl}_{3}\right)$

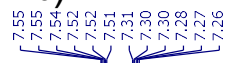

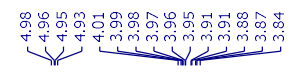
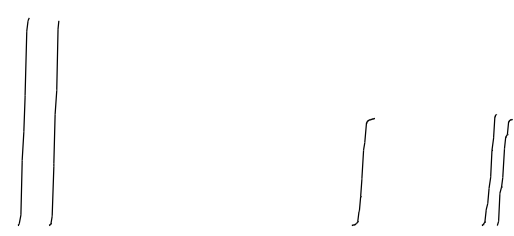<smiles>ClCC(Cl)c1ccc(Br)cc1</smiles>

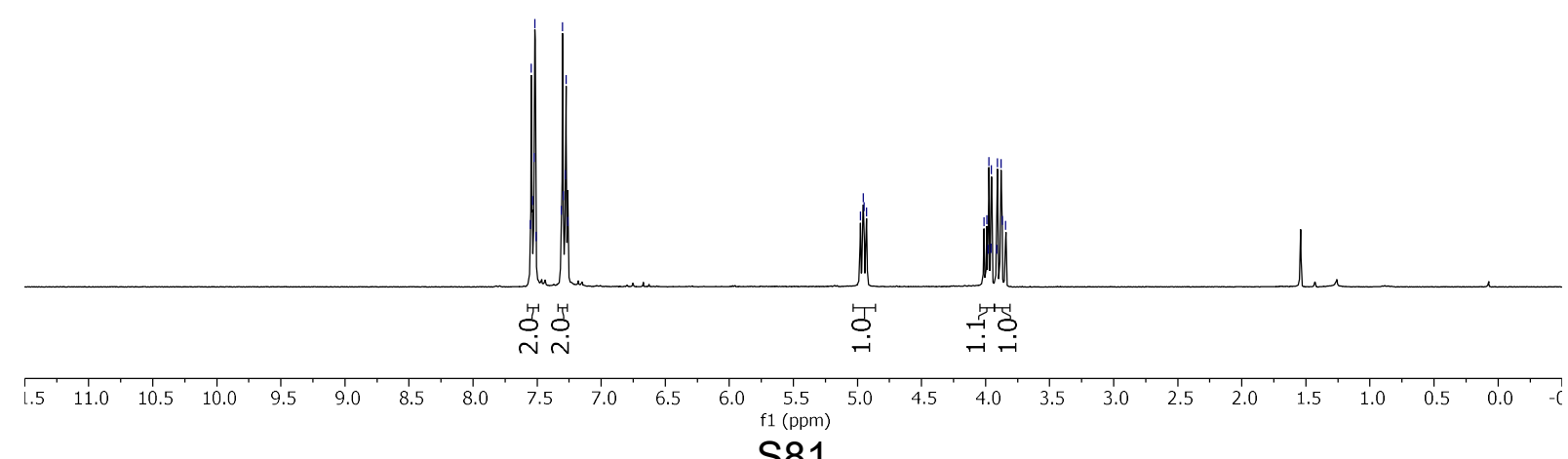


2-(1,2-dichloroethyl)-1,1'-biphenyl (16)

${ }^{1} \mathrm{H} \mathrm{NMR}\left(300 \mathrm{MHz} \mathrm{CDCl}_{3}\right)$

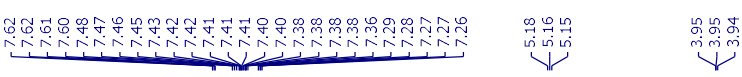<smiles>ClCC(Cl)c1ccccc1-c1ccccc1</smiles><smiles>C1#CC1</smiles><smiles>C1CC1</smiles>

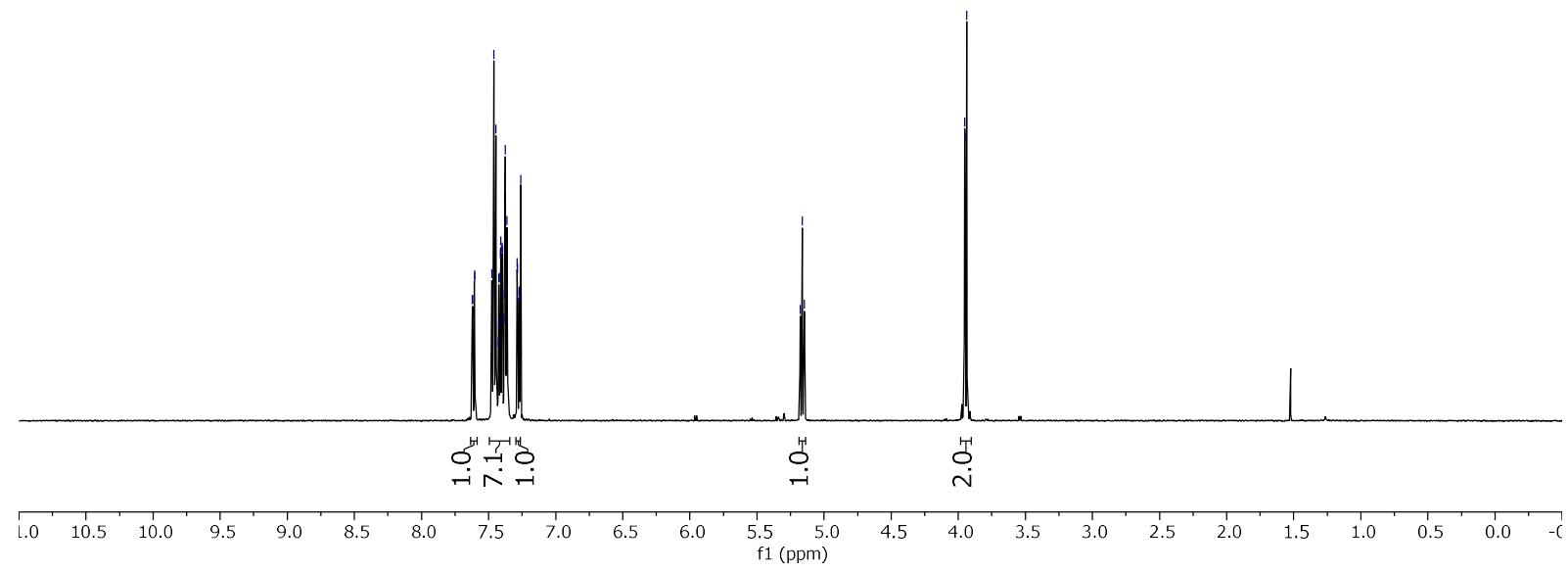




\section{4,5-Dichlorohexyl benzoate (17)}

${ }^{1} \mathrm{H}$ NMR $\left(400 \mathrm{MHz} \mathrm{CDCl}_{3}\right)$

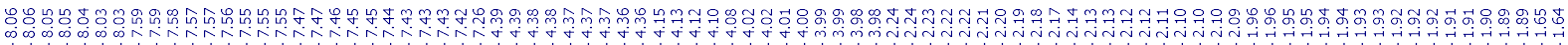
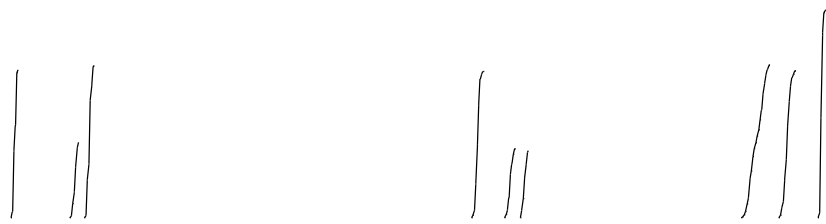<smiles>C[C@H](Cl)[C@H](Cl)CCCOC(=O)c1ccccc1</smiles>
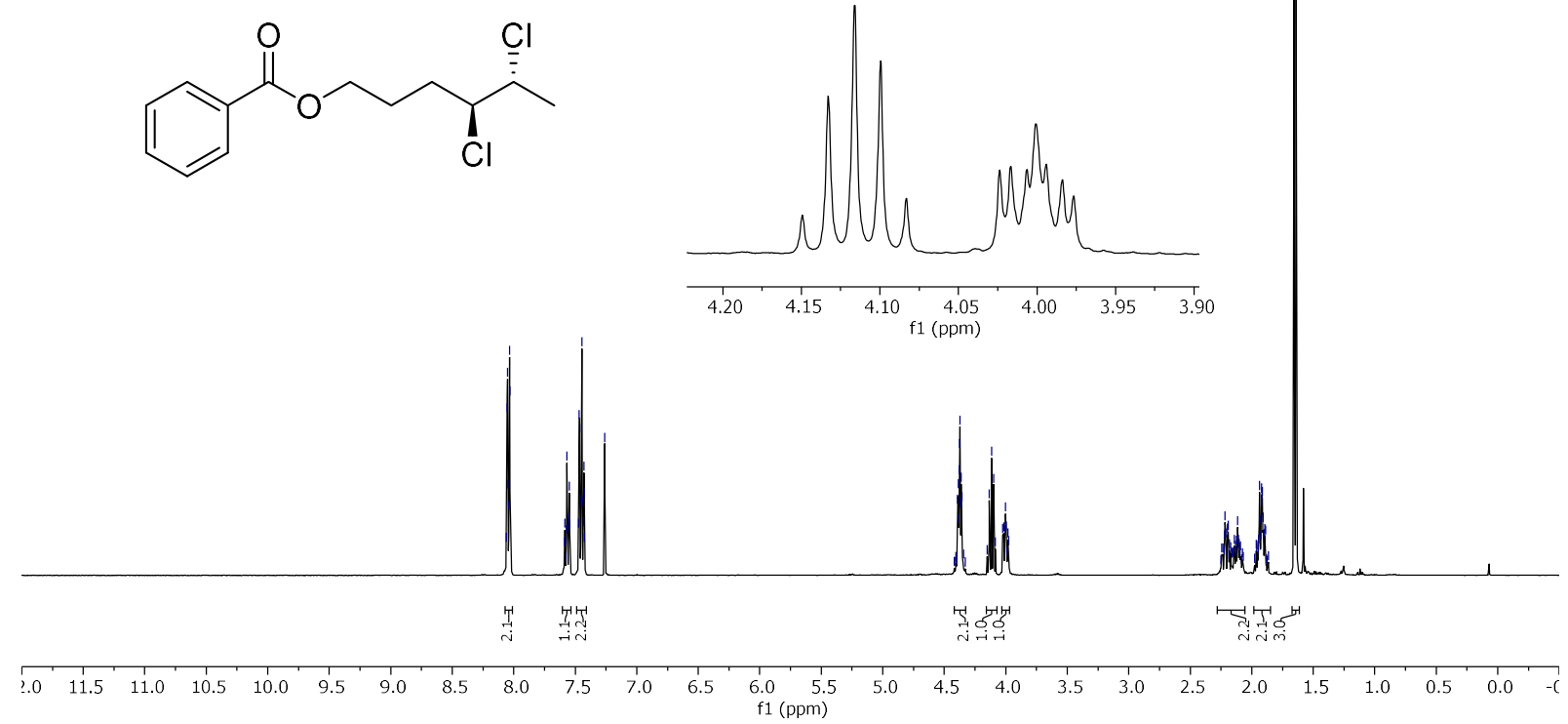

${ }^{13} \mathrm{C}$ NMR $\left(101 \mathrm{MHz}, \mathrm{CDCl}_{3}\right)$

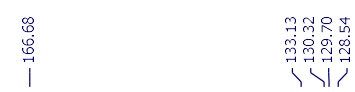<smiles>CC(Cl)C(Cl)CCCOC(=O)c1ccccc1</smiles>

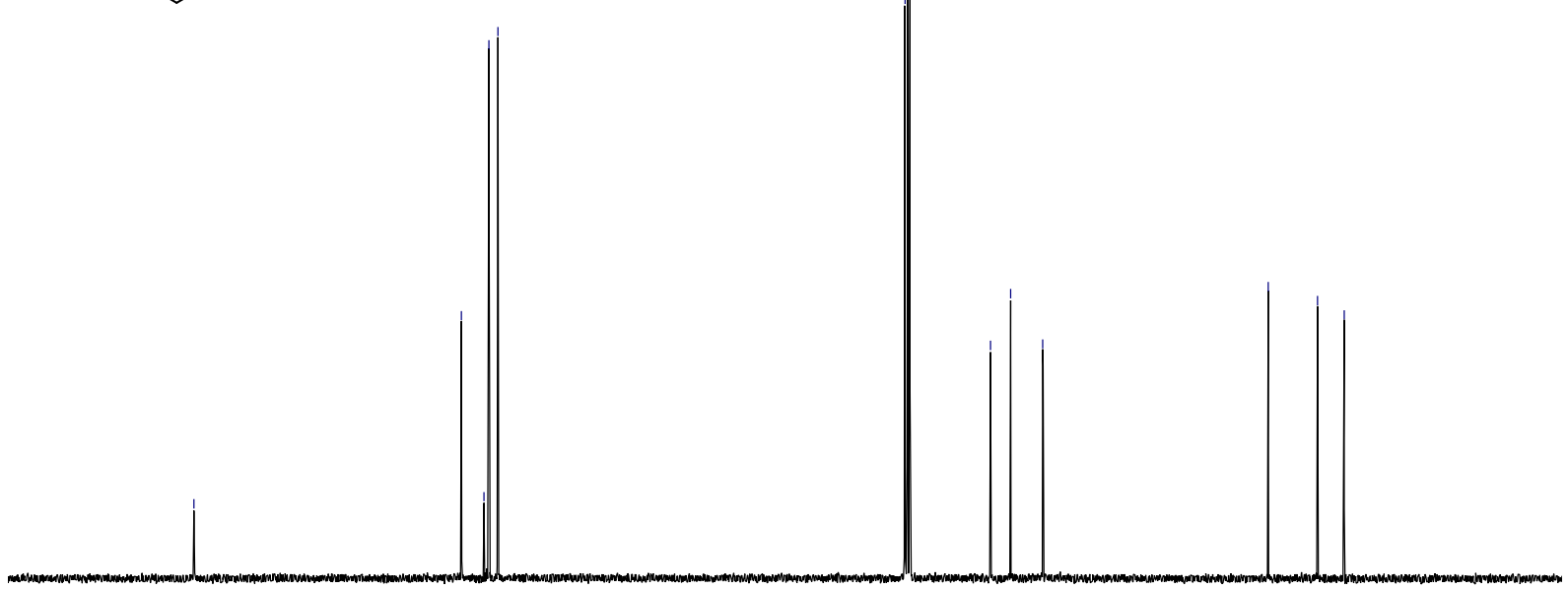




\section{4,5-Dichlorohexyl 4-methoxybenzoate (18)}

${ }^{1} \mathrm{H} \mathrm{NMR}\left(400 \mathrm{MHz} \mathrm{CDCl}_{3}\right)$

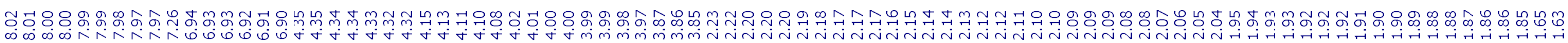
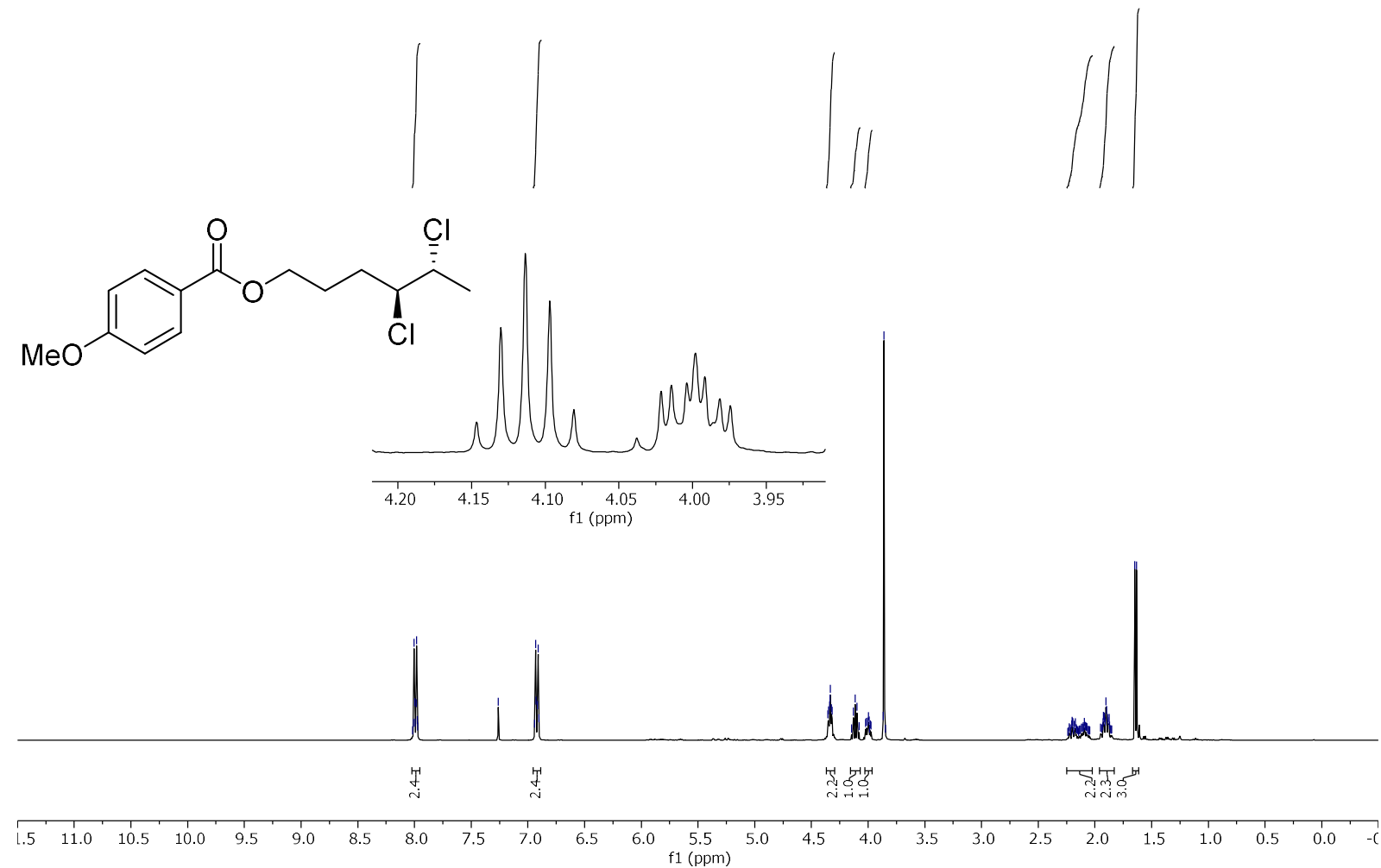

${ }^{13} \mathrm{C}$ NMR $\left(101 \mathrm{MHz}, \mathrm{CDCl}_{3}\right)$

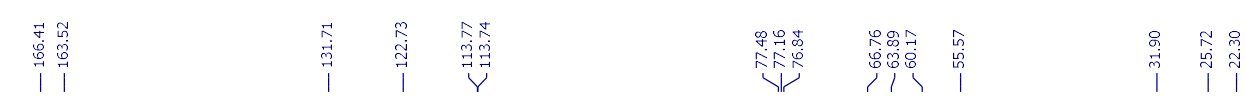<smiles>COc1ccc(C(=O)OCCCC(Cl)[C@H](C)Cl)cc1</smiles>
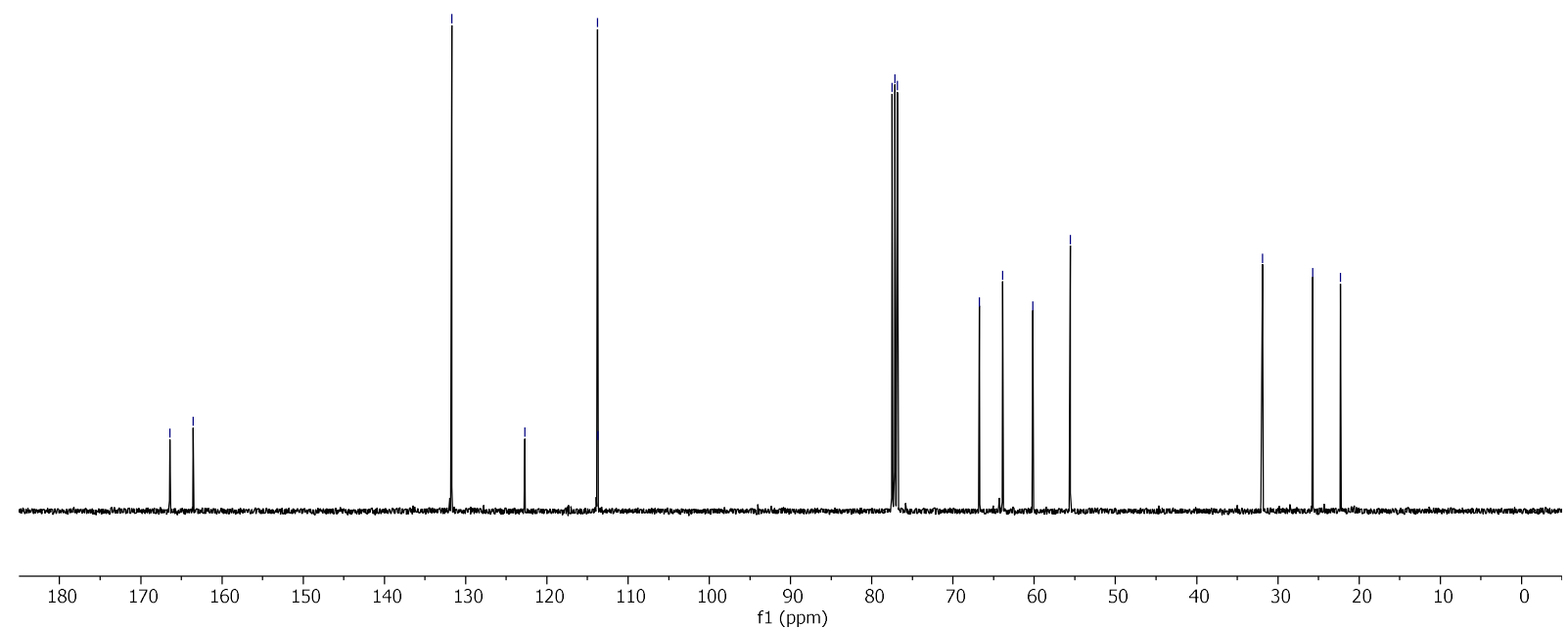


\section{4,5-Dichlorohexyl 3,5-dinitrobenzoate (19)}

${ }^{1} \mathrm{H} \mathrm{NMR}\left(400 \mathrm{MHz} \mathrm{CDCl}_{3}\right)$

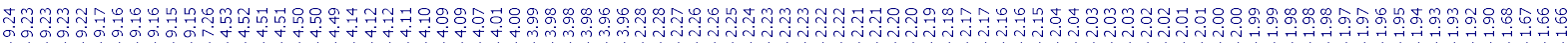<smiles>C#CCCCCC</smiles><smiles>CC(Cl)C(Cl)CCCOC(=O)c1cc([N+](=O)[O-])cc([N+](=O)[O-])c1</smiles>
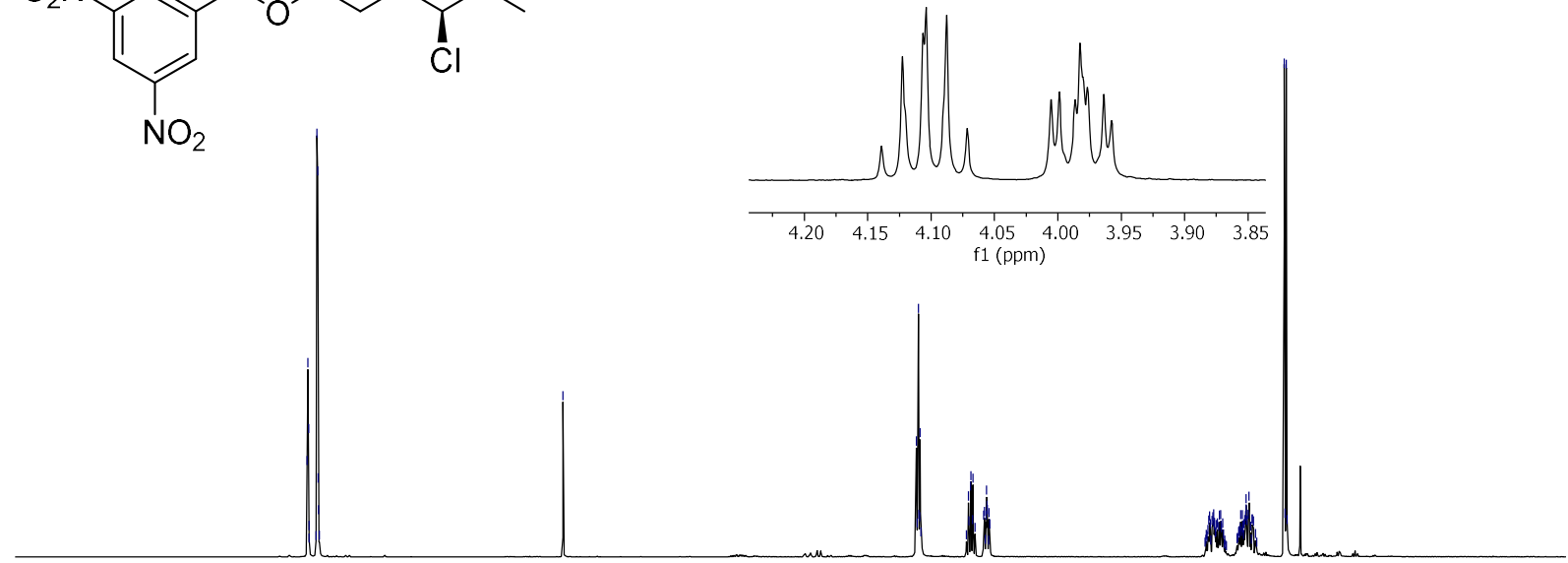

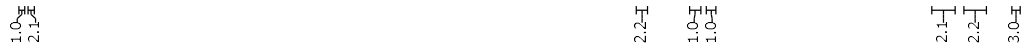

${ }^{3} \mathrm{C}$ NMR (101 $\left.\mathrm{MHz}, \mathrm{CDCl}\right)$

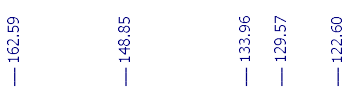<smiles>CC(Cl)C(Cl)CCCOC(=O)c1cc([N+](=O)[O-])cc([N+](=O)[O-])c1</smiles>

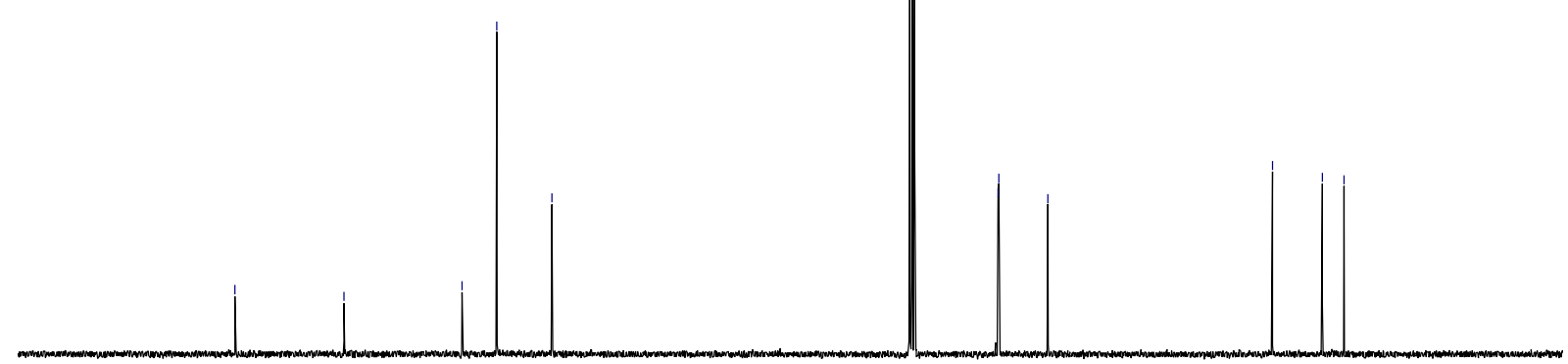




\section{4,5-Dichlorohexyl benzoate (20)}

${ }^{1} \mathrm{H} \mathrm{NMR}\left(400 \mathrm{MHz} \mathrm{CDCl}_{3}\right)$

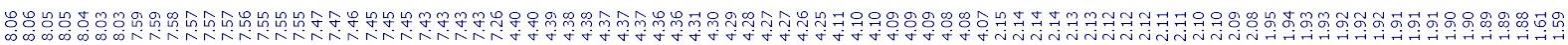
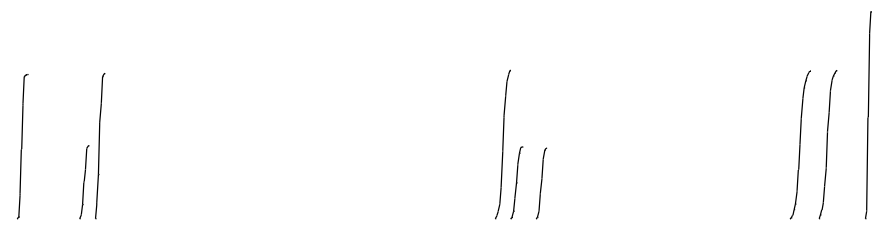<smiles>CC(Cl)C(Cl)CCCOC(=O)c1ccccc1</smiles>
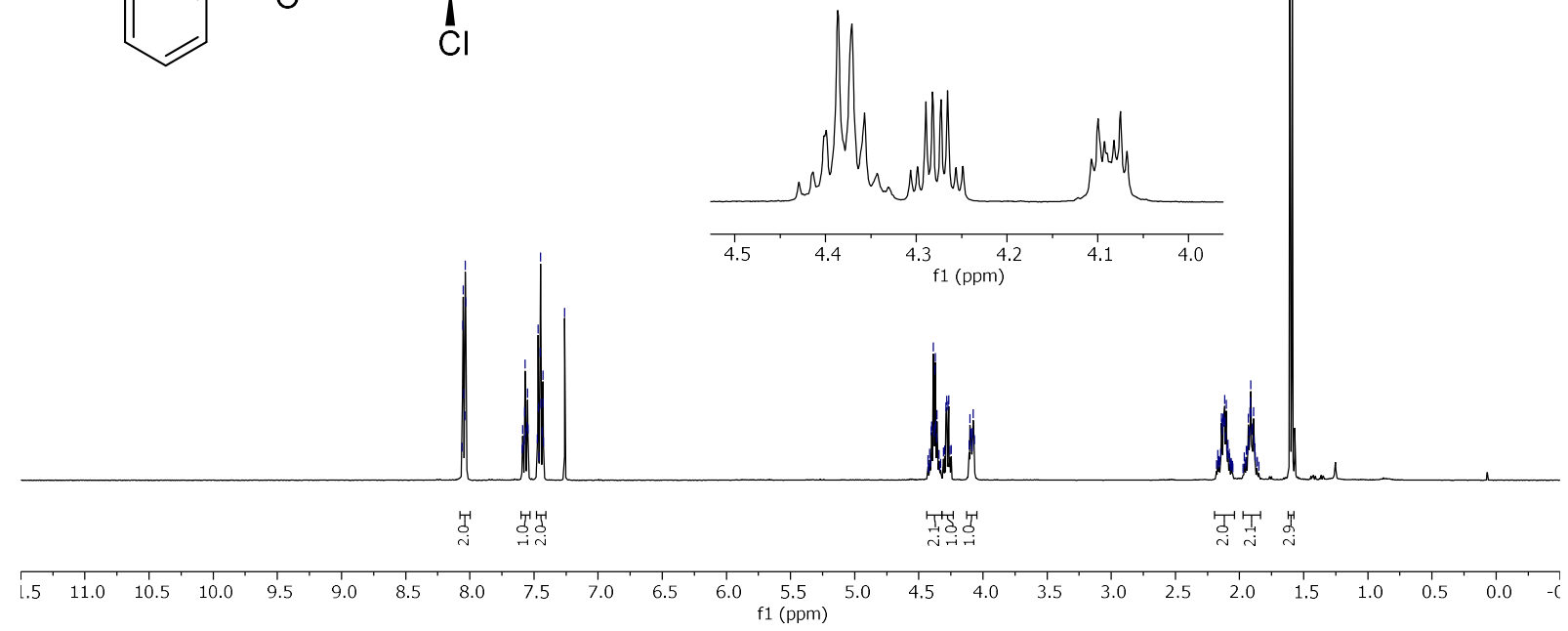

${ }^{13} \mathrm{C}$ NMR (101 MHz, CDCl 3 )

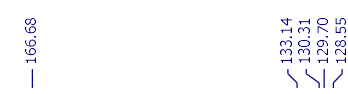<smiles>CC(Cl)C(Cl)CCCOC(=O)c1ccccc1</smiles>
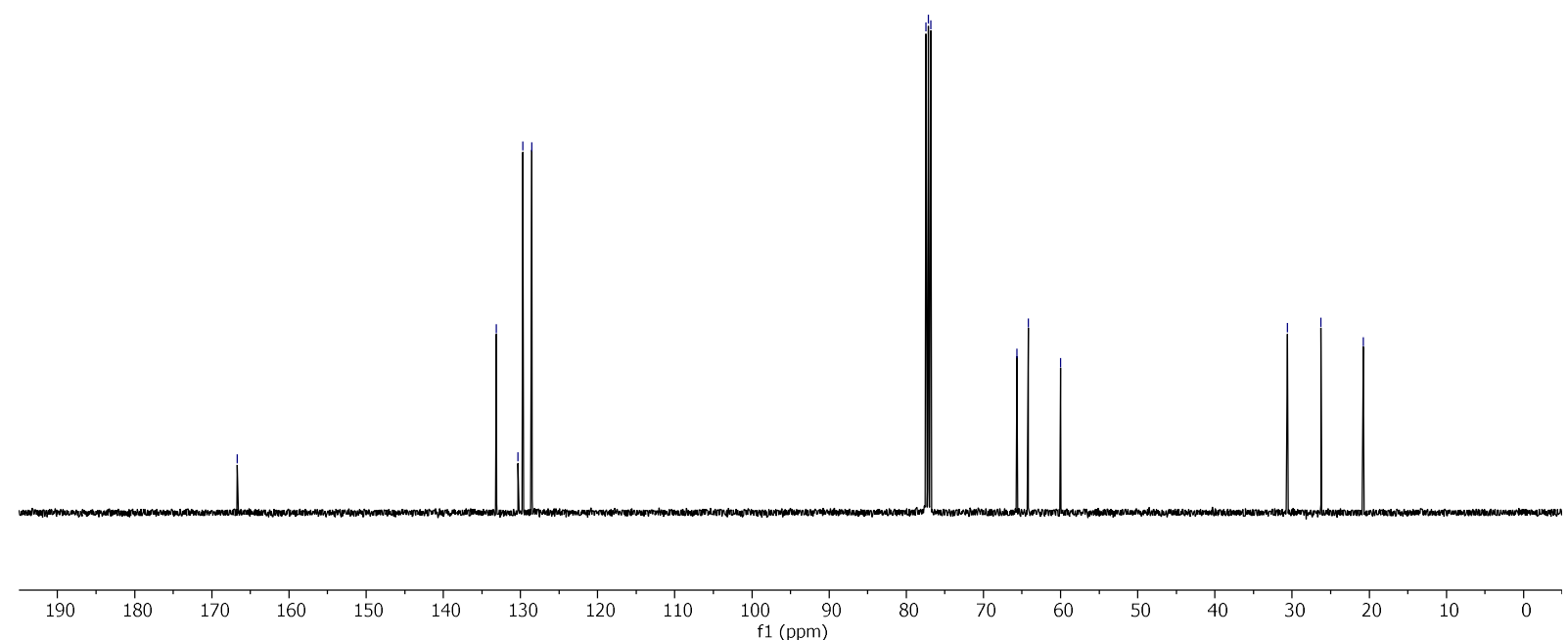


\section{4,5-Dichlorohexyl 3,5-dinitrobenzoate (21)}

${ }^{1} \mathrm{H} \mathrm{NMR}\left(400 \mathrm{MHz} \mathrm{CDCl}_{3}\right)$

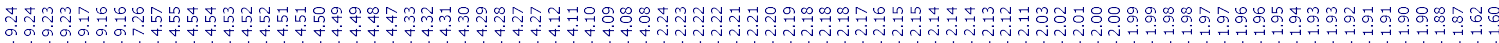

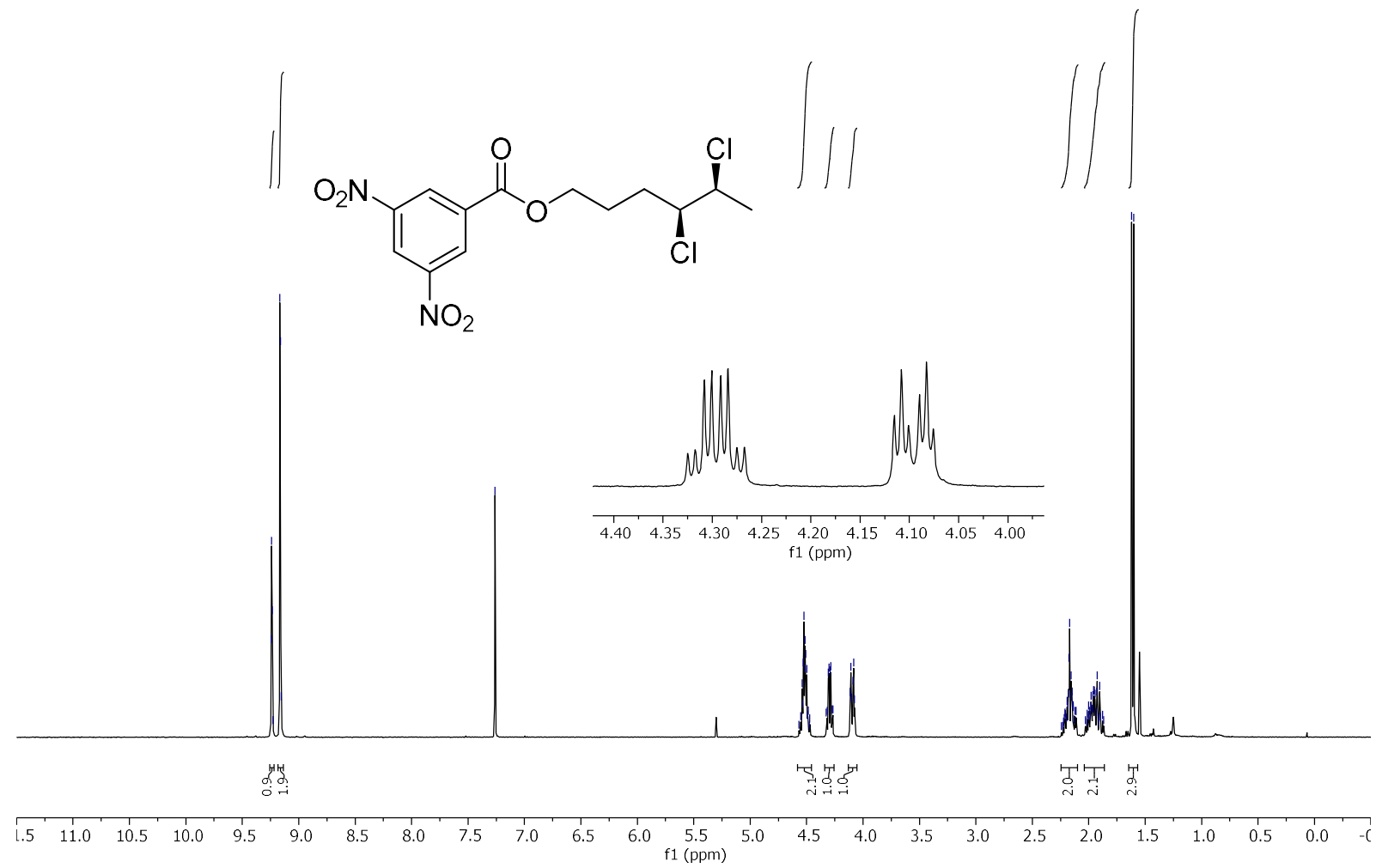

$\left.{ }^{13} \mathrm{C} \mathrm{NMR} \mathrm{(101} \mathrm{MHz,} \mathrm{CDCl}_{3}\right)$

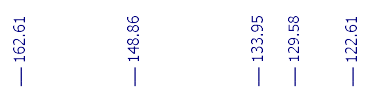

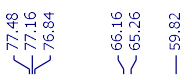<smiles>CC(Cl)C(Cl)CCCOC(=O)c1cc([N+](=O)[O-])cc([N+](=O)[O-])c1</smiles>

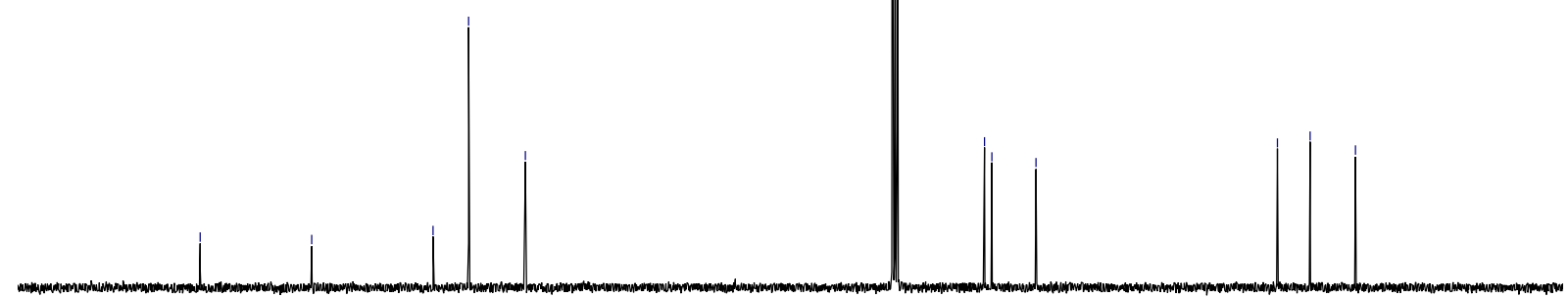


$\mathrm{N}$-allyl-N-(2,3-dichloropropyl)-4-methylbenzenesulfonamide (22)

${ }^{1} \mathrm{H}$ NMR $\left(400 \mathrm{MHz}, \mathrm{CDCl}_{3}\right)$

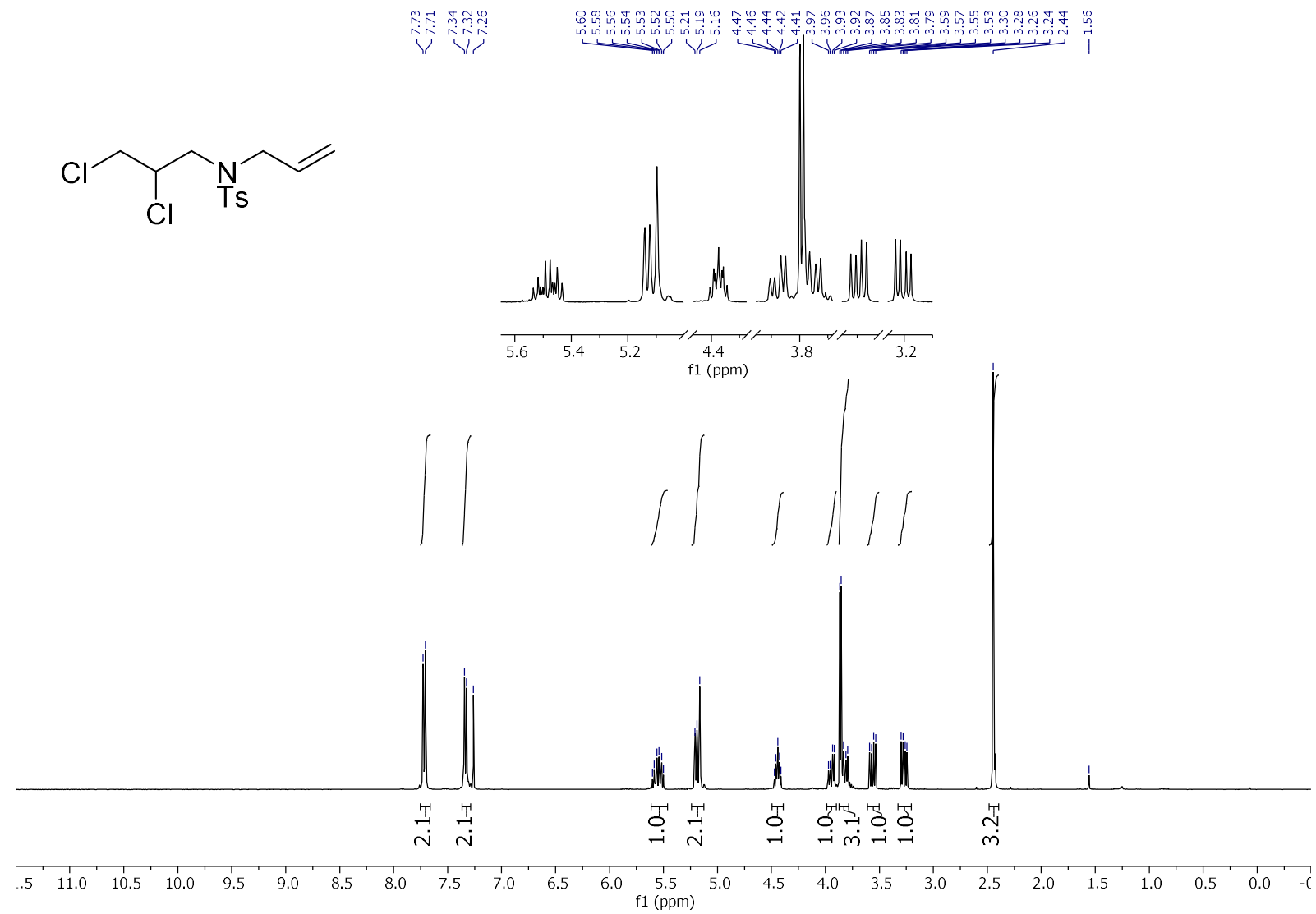

$\mathbf{N}, \mathbf{N}$-bis(2,3-dichloropropyl)-4-methylbenzenesulfonamide (23) ${ }^{1} \mathrm{H}$ NMR $\left(400 \mathrm{MHz}, \mathrm{CDCl}_{3}\right)$

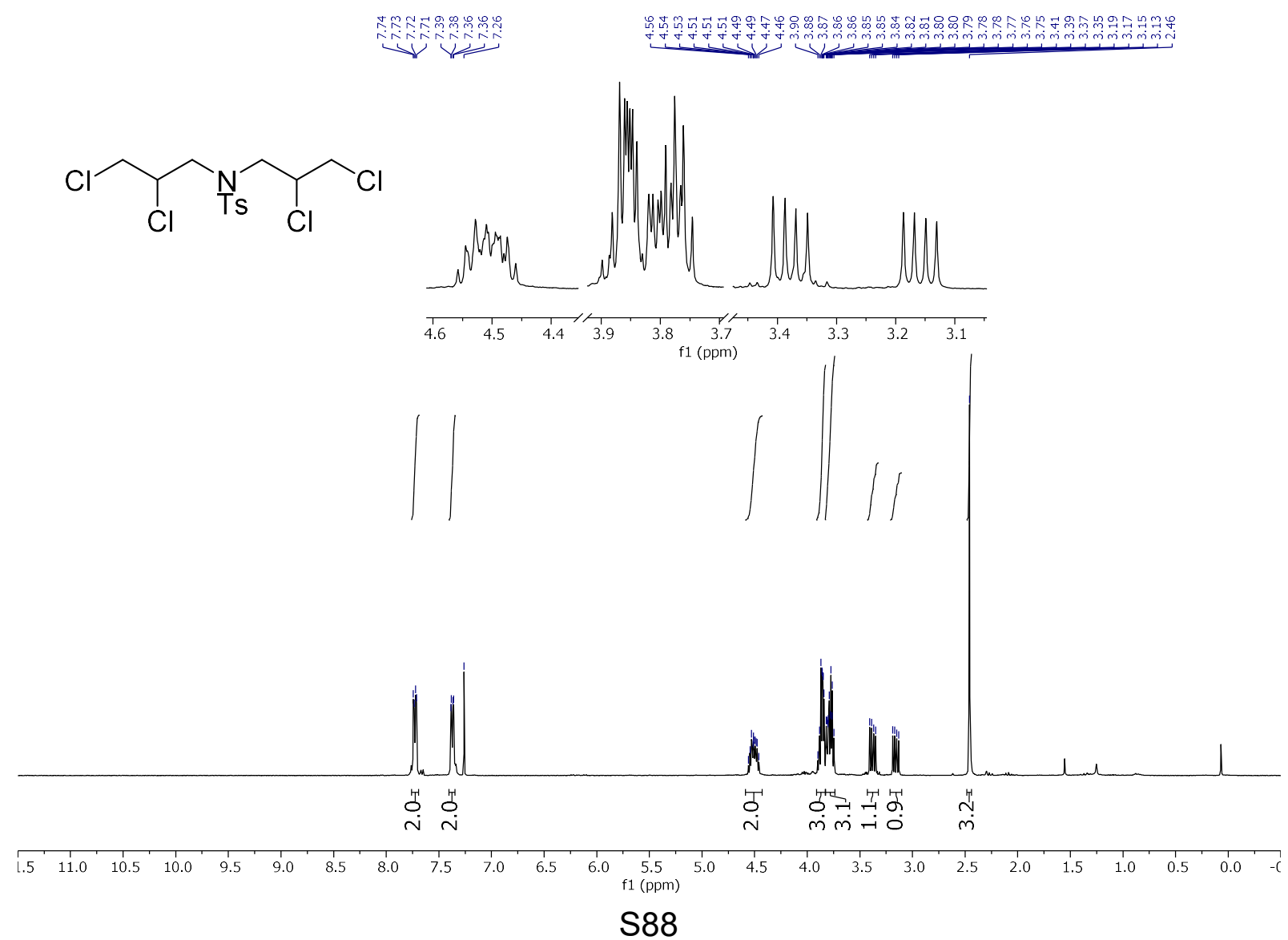


XII. NMR Spectra: preliminary validation of an enantioselective variant (Di((3S,5S,7S)-adamantan-1-yl) 2,2'-((2-iodo-5-methyl-1,3-phenylene)bis(oxy)) $\left(2 R, 2^{\prime} R\right)$-dipropionate (S20) ${ }^{1} \mathrm{H}$ NMR (400 $\mathrm{MHz} \mathrm{CDCl}_{3}$ )

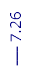

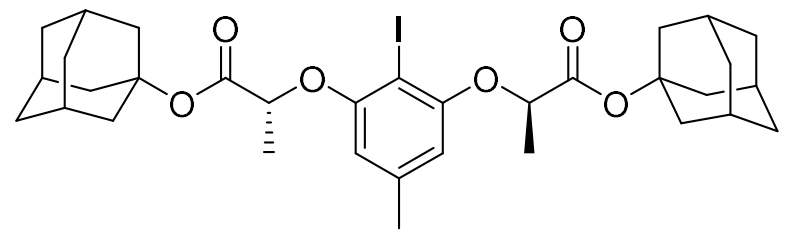

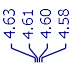

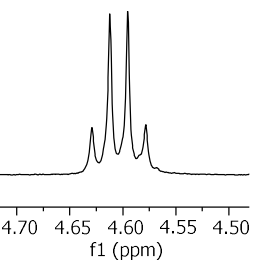

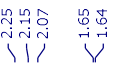

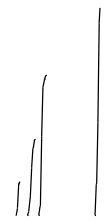

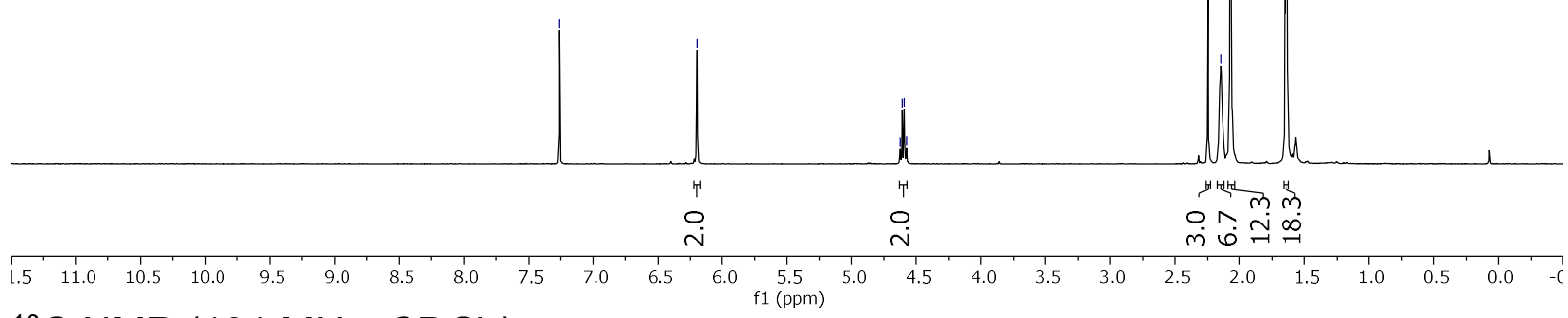

${ }^{13} \mathrm{C}$ NMR $\left(101 \mathrm{MHz}, \mathrm{CDCl}_{3}\right)$
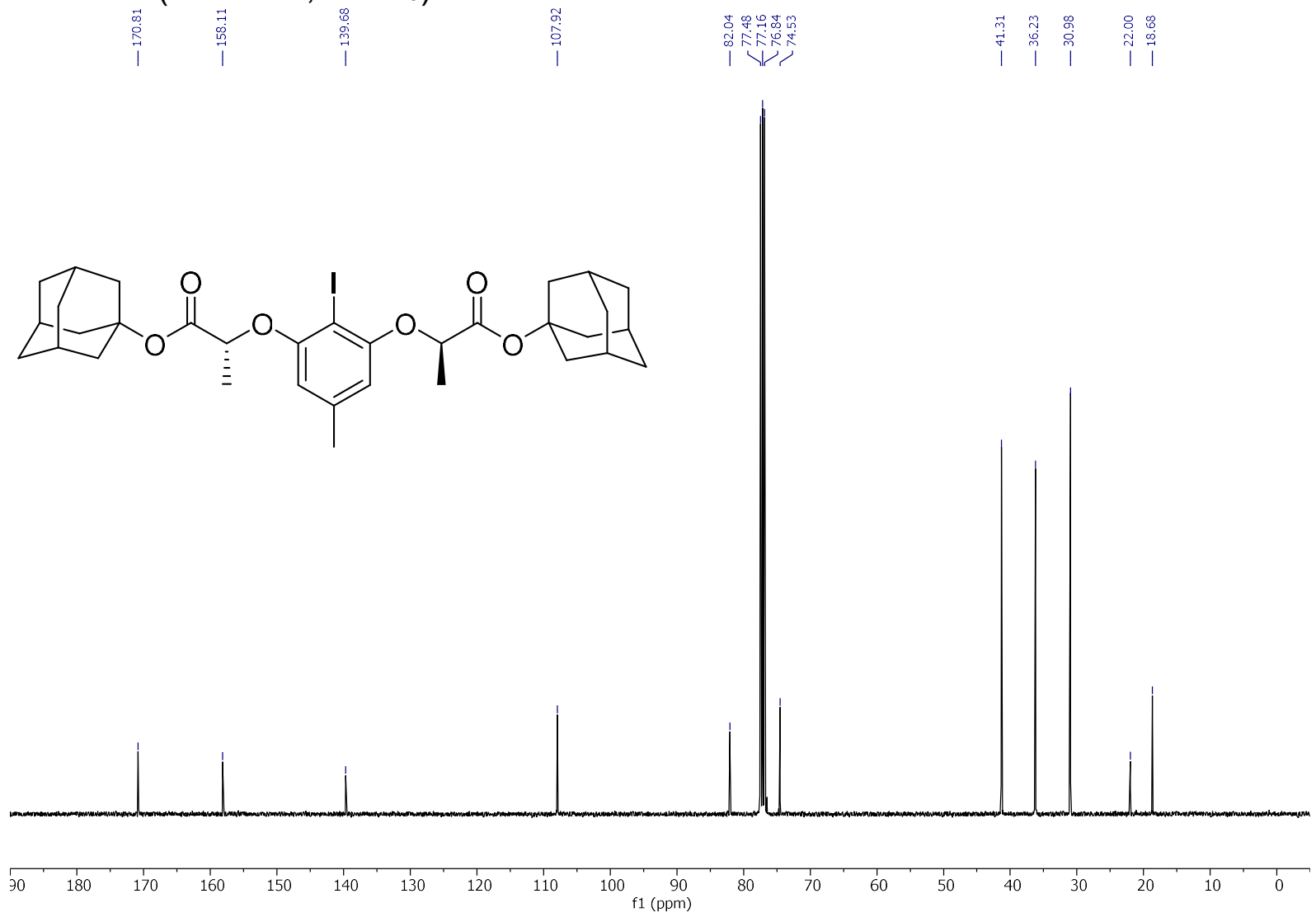

S89 


\section{References}

${ }^{1}$ L. Ren, M.-M. Yang, C.-H. Tung, L.-Z. Wu, H. Cong ACS Catal. 2017, 7, 8134-8138.

2 Y. Liu, H. Wu, Y. Guo, J.-C. Xiao, Q.-Y. Chen, C. Liu Angew. Chem. Int. Ed. 2017, 56, 15432-15435.

${ }^{3}$ T. Sastraruji, S. G. Pyne, A. T. Ung Tetrahedron 2012, 68, 598-602.

${ }^{4}$ I. Buslov,J. Becouse, S. Mazza, M. Montandon-Clerc, X. Hu, Angew. Chem. Int. Ed. 2015, 54, 1452314526; Angew. Chem. 2015, 127, 14731-14734.

5 I. G. Molnár, R. Gilmour J. Am. Chem. Soc. 2016, 138, 5004-5007.

${ }^{6}$ L. Ying, L. Xiaohui, L. Xiuru, J. Zhan, L. Yuesheng, J Polym Sci Part A: Polym Chem. 2006, 45, $26-$ 40.

${ }^{7}$ K. Yasumoto, A. Nishigami, H. Aoi, C. Tsuchihashi, F. Kasai, T. Kusumi,T. Ooi Chem. Pharm. Bull. 2008, 56, 133-136.

${ }^{8}$ R. K. Dieter, V. K. Gore, N. Chen Org. Lett. 2004, 6, 763-766.

${ }^{9}$ A. Jana, K. Misztal, A. Zak, K. Grela J. Org. Chem. 2017, 82, 4226-4234.

10 W. N. White, D. Gwynn, R. Schlitt, C. Girard, W. Fife J. Am. Chem. Soc. 1958, 80, 3271-3277.

11 J. G. Alauzun, J. N. Fortuna, H. Sheardown, F. Gonzaga, M. A. Brook J. Mater. Chem. 2009, 19, 50335038.

12 C. M. So, S. Kume, T. Hayashi J. Am. Chem. Soc. 2013, 135, 10990-10993.

13 J. Tao, R. Tran, G. K. Murphy J. Am. Chem. Soc. 2013, 135, 16312-16315.

14 W. B. Motherwell, M. F. Greaney, D. A. Tocher J. Chem. Soc., Perkin Trans. 1 2002, 0, 2809.

${ }^{15}$ N. Fu, G. S. Sauer, S. Lin J. Am. Chem. Soc. 2017, 139, 15548-15553.

${ }^{16}$ S. Thurnhofer, K. Lehnert, W. Vetter Eur. Food Res. Technol. 2008, 226, 975-983.

${ }^{17}$ P. Swamy, M. M. Reddy, M. A. Kumar, M. Naresh, N. Narender Synthesis 2014, 46, 251-257.

18 Z. Zhao, I. Jameel, G. K. Murphy Synthesis 2019, 51, 2648-2659.

${ }^{19} \mathrm{~K}$. Uchida, S. Yoshida, T. Hosoya, Synthesis 2016, 48, 4099-4109.

20 S. Haubenreisser, T. H. Wöste, C. Martinez, K. Ishihara, K. Muniz Angew. Chem. Int. Ed. 2016, 55, 413-417.

${ }_{21}$ M. Shimogaki, M. Fujita, T. Sugimura Eur. J. Org. Chem. 2013, 7128-7138.

22 M. Uyanik, T. Yasui, K. Ishihara Angew. Chem. Int. Ed. 2013, 52, 9215-9218.

${ }^{23}$ T. Wöste, K. Muñiz Synthesis 2016, 48, 816-827. 